\title{
STRATEGY-DRIVEN IMPLICATIONS FOR THE MANAGEMENT CONTROL SYSTEMS OF ELECTRICITY GENERATORS DUE TO GOVERNMENT CLIMATE CHANGE POLICIES
}

\section{BY \\ BINH THANH BUI}

\begin{abstract}
A thesis
submitted to the Victoria University of Wellington

in fulfilment of the requirements for the degree of

Doctor of Philosophy
\end{abstract}

Victoria University of Wellington

2011 


\section{ABSTRACT}

The New Zealand Government has developed and revised different climate change policies (GCCPs) over the last nine years in order to meet its Kyoto obligations and address the challenges of climate change. The key GCCPs are the carbon tax proposed in 2002 but cancelled in 2005; the Projects to Reduce Emissions (PRE) scheme during 2003-2004; and an emissions trading scheme (ETS) announced in 2007, legislated in 2008 and then modified in 2009. These GCCP changes are likely to impose significant costs and additional volatilities and pressures on electricity generators because their production activities are emissions-intensive. Consequently generators have been the direct targets of various GCCPs.

This study addresses the following research question: "What are the management control system (MCS) implications arising from strategy choices New Zealand electricity generators make in managing economic volatility and societal pressures resulting from a prolonged period of GCCP change?". In answering this question, the study adopts a multiple-theory research framework that builds on insights from prior literature and the theoretical perspectives of transactional cost economics, institutional theory and resource-based theory. This study uses a multiple-case study that analyses interviews and 10-years of documentary data related to five major generators within the New Zealand electricity industry over five periods from 2000 to 2009.

The results suggest that GCCP changes impose external economic volatilities and societal pressures on the generators and affect their costs, market competitiveness, and social legitimacy. The diversity of internal characteristics and capabilities of the generators are found to moderate their relative degree of exposure to these external volatilities and pressures. Ten GCCP-related environmental strategies were formulated and implemented by the generators, with different levels of proactiveness (i.e. scope and timing), varying top management emphasis, as well as distinct underlying objectives. These strategy choices had significant implications for organisational MCS. In response, a wide range of controls and control systems were adopted. These can be classified into eight MCS component types and three MCS component groups, each of which supported one or more environmental strategies. Managers varied the degree of use of these MCS component types and groups to match the level of strategy proactiveness and top management emphasis pursued within each period. Additionally, the organisational MCS was underlined by three main objectives (cost control, competitiveness, and 
legitimacy) which in turn were driven by each generator's primary strategy objectives and GCCP-related external exposure.

This study addresses a number of gaps in the academic literature and demonstrates the merits of a multiple-theoretical framework in examining GCCP-related strategy and MCS changes. The results also have significant implications for managers and practitioners when planning organisational adaptation to a carbon-constrained economy. Further, the study provides a useful basis for regulators and policy-makers in making the appropriate assessment and providing advice to improve the GCCPs' environmental and economic outcomes. 


\section{ACKNOWLEDGEMENT}

I would like to express my sincerest thanks to my supervisors, Dr Carolyn Fowler and Associate Professor Chris Hunt, for their devoted academic guidance and support throughout the process of my PhD study. I am also indebted to Professor Tony van Zijl, Professor Keitha Dunstan, and Professor Brenda Porter for their initial assistance on the choice of $\mathrm{PhD}$ topic. My gratitude also goes to the staff of the School of Accounting and Commercial Law and $\mathrm{PhD}$ colleagues, who have given me invaluable encouragement and advice regarding my thesis.

And foremost, thank you, Quan, for being the most dedicated, trusting and inspirational husband and for simply being there when I need you. 


\section{TABLE OF CONTENTS}

\section{CHAPTER 1: INTRODUCTION}

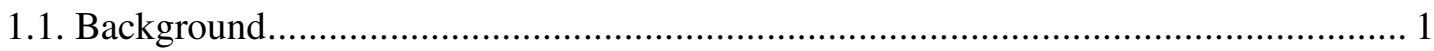

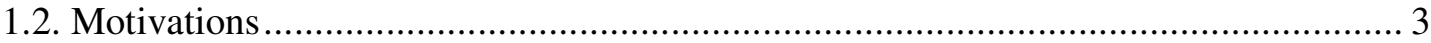

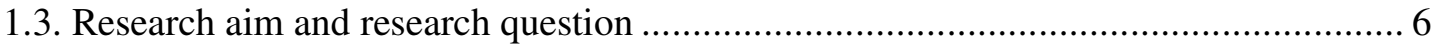

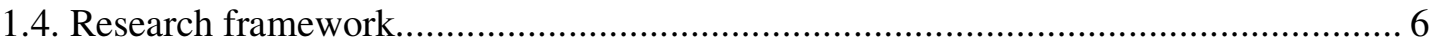

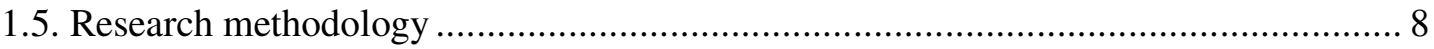

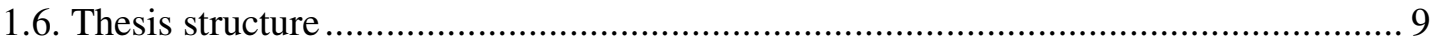

\section{CHAPTER 2: THE GOVERNMENT CLIMATE CHANGE POLICIES AND} ELECTRICITY GENERATORS

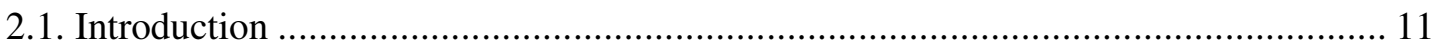

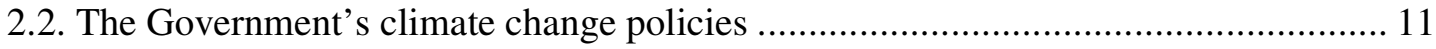

2.3. Electricity generators and the potential impacts of the GCCPs................................ 15

2.4. Potential organisational strategic and MCS responses to the GCCPs ....................... 22

2.5. Practical motivations to this study and the research question ................................ 24

\section{CHAPTER 3: ENVIRONMENTAL STRATEGIES AND MCS}

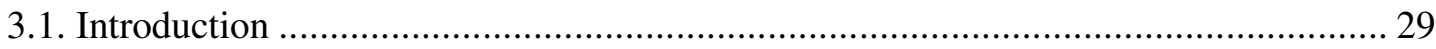

3.2. External drivers of environmental strategies and MCS ....................................... 29

3.3. Internal drivers of environmental strategies: internal capabilities and characteristics 34

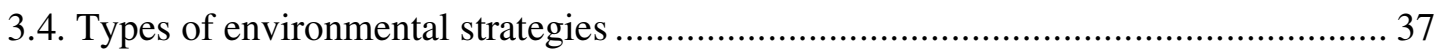

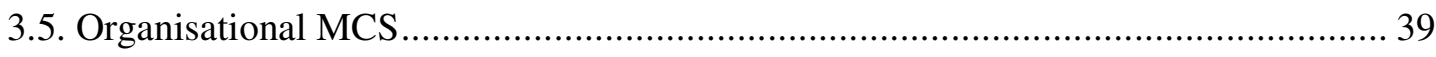

3.6. Evaluation of prior literature and identification of remaining gaps......................... 45

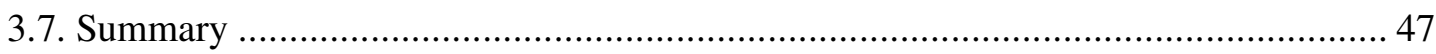

\section{CHAPTER 4: RESEARCH FRAMEWORK}

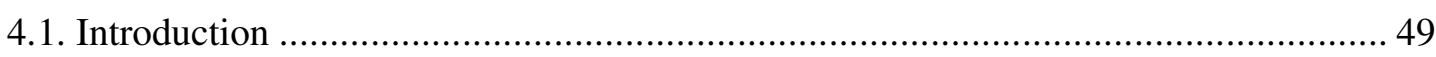

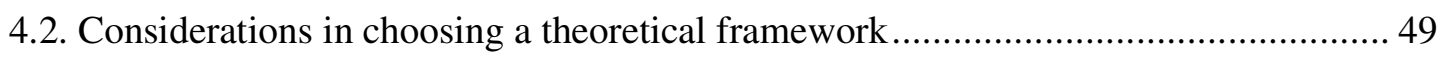

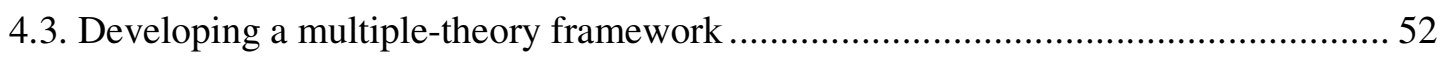

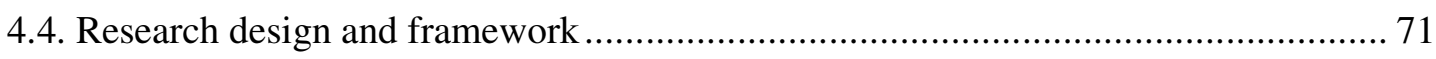

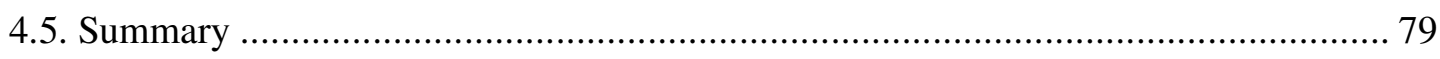

\section{CHAPTER 5: RESEARCH METHODOLOGY}

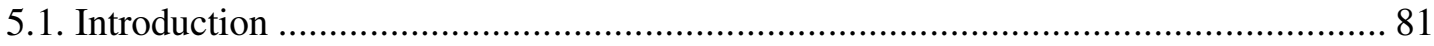

5.2. A longitudinal multiple-case study approach (LMC) ...................................... 81 


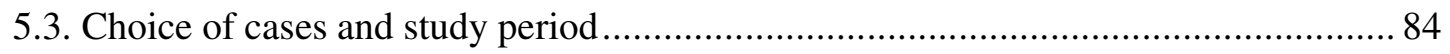

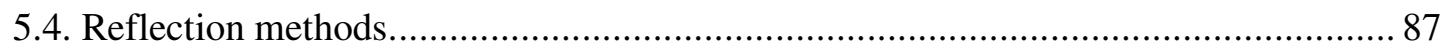

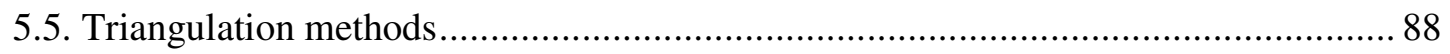

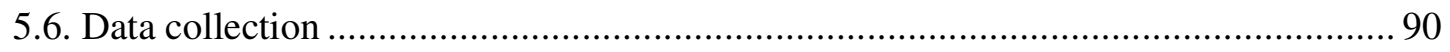

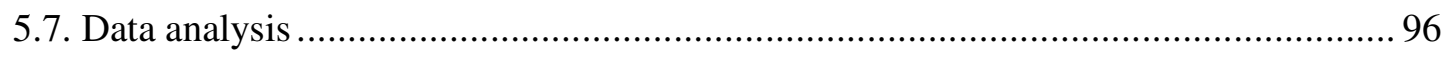

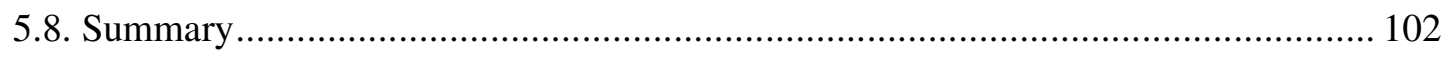

CHAPTER 6: FIRM-BASED EVIDENCE OF ENVIRONMENTAL STRATEGIES

AND ORGANISATIONAL MCS (2000 - 2009)

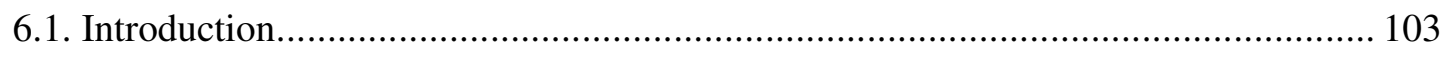

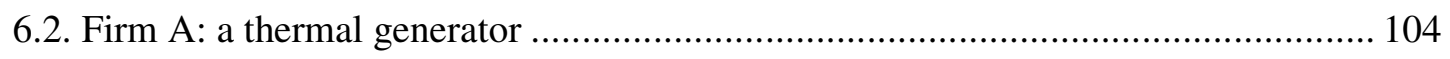

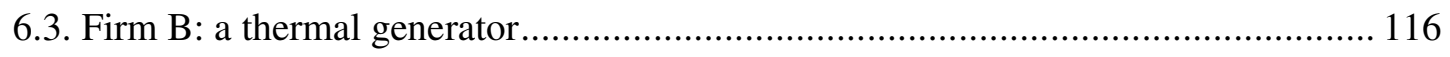

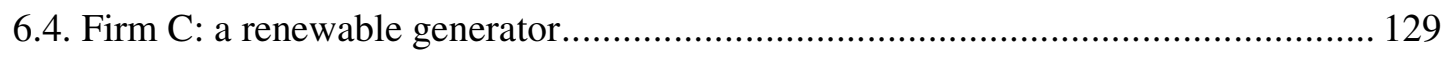

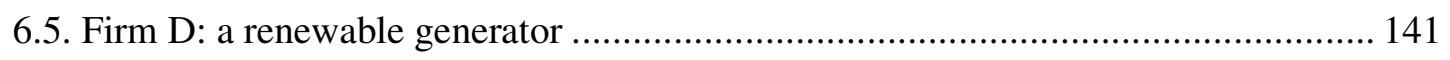

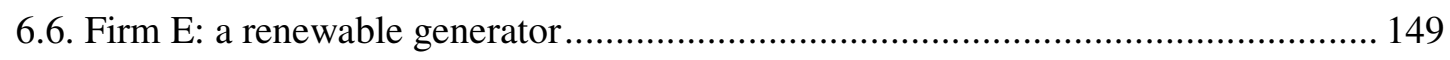

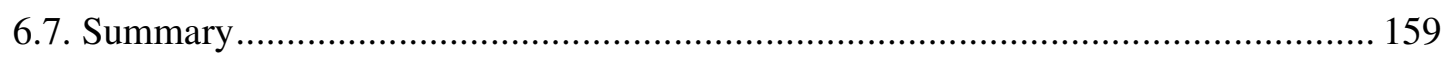

CHAPTER 7: GCCP-RELATED EXTERNAL AND INTERNAL DRIVERS AND

THEIR IMPACTS ON ENVIRONMENTAL STRATEGIES

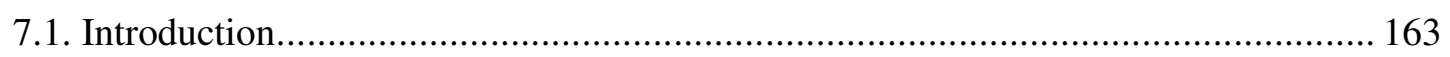

7.2. Overview of the five generators and the types of environmental strategies.............. 164

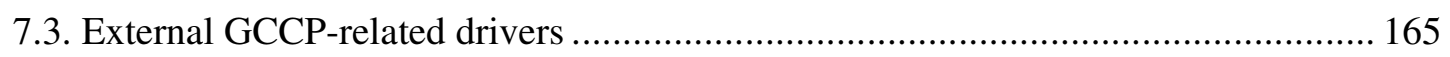

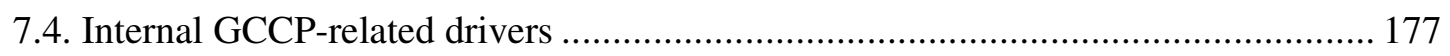

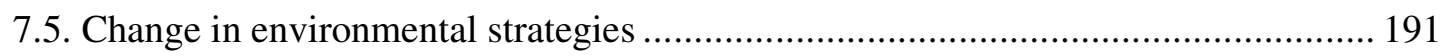

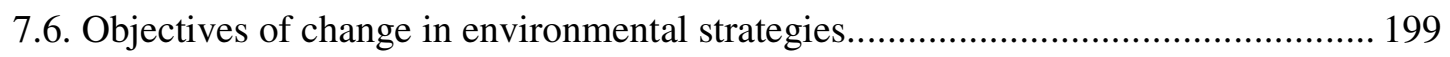

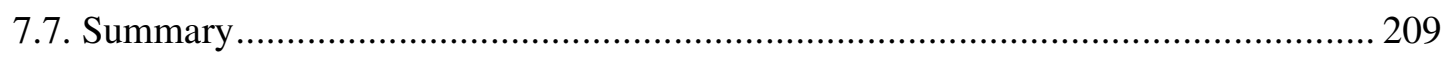

CHAPTER 8: CHANGE IN ORGANISATIONAL MCS

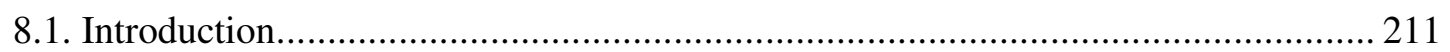

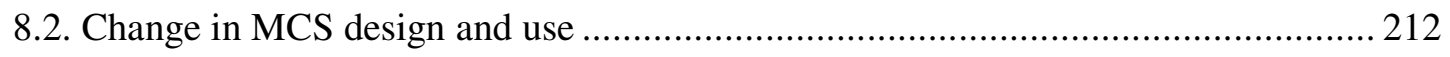

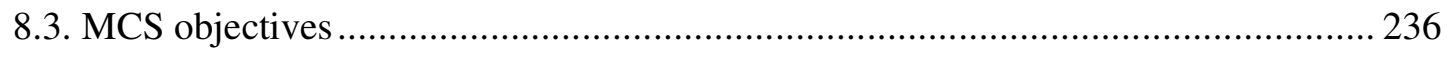

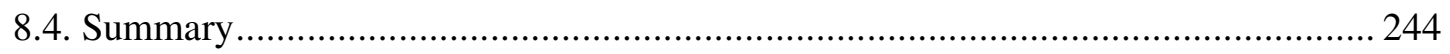

\section{CHAPTER 9: DISCUSSION AND CONCLUSION}

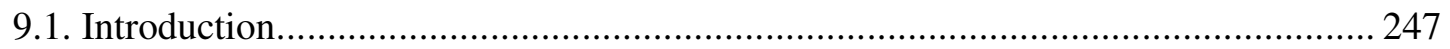

9.2. Research question - implications for organisational MCS .................................... 248

9.3. Validation of the research framework and propositions ........................................2250

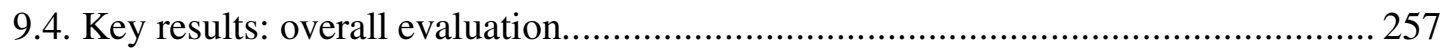




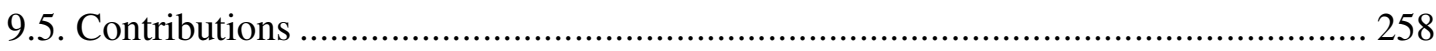

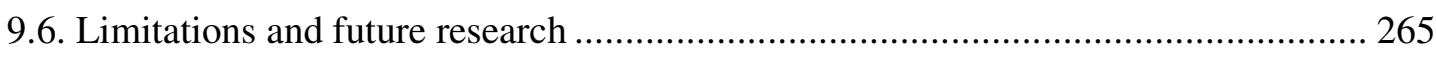

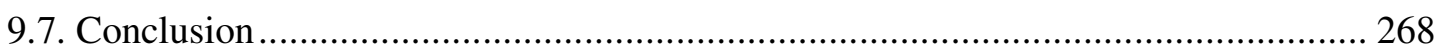

\section{APPENDICES}

Appendix A: proposal for research participation .............................................. 271

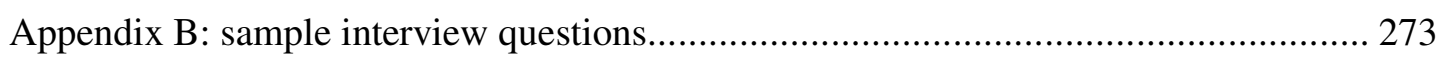

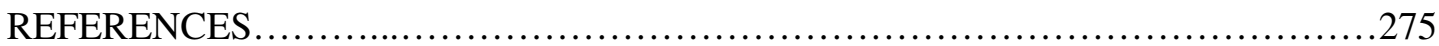




\section{LIST OF TABLES AND FIGURES}

\section{TABLES}

Table 5-1: Variations in organisation-level characteristics of electricity generators .............92

Table 5-2: Matrix data display for GCCP-related external drivers ....................................98

Table 5-3: Matrix data display for GCCP-related internal characteristics.............................98

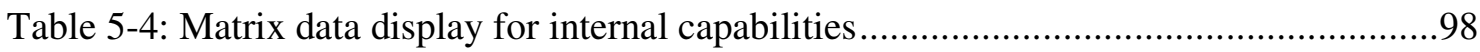

Table 5-5: Matrix data display for strategy proactiveness ..............................................99

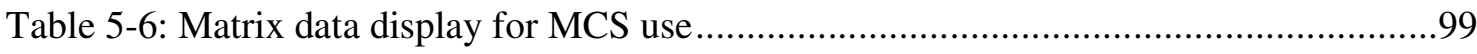

Table 6-1: Count of Firm A-related evidence availability ..............................................115

Table 6-2: Count of Firm B-related evidence availability .............................................128

Table 6-3: Count of Firm C-related evidence availability ..............................................141

Table 6-4: Count of Firm D-related evidence availability ..............................................148

Table 6-5: Count of Firm E-related evidence availability ................................................158

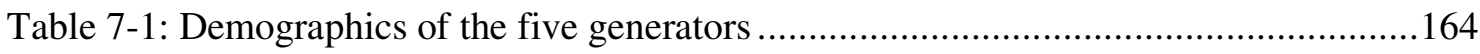

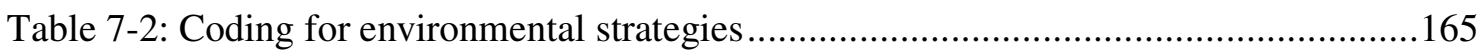

Table 7-3: External economic volatilities and their impacts on environmental strategies

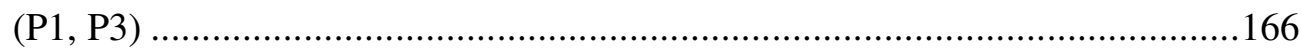

Table 7-4: External institutional pressures and their impacts on environmental strategies

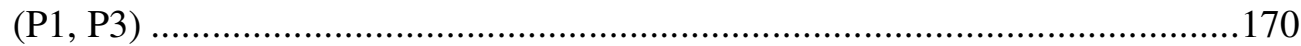

Table 7-5: Internal characteristics and their impacts on environmental strategies (P2, P3)..177

Table 7-6: Internal capabilities and their impacts on environmental strategies (P2, P3) ......184

Table 7-7: Change in strategic emphasis of five generators (2000-2009) (P3) ...................197

Table 7-8: Strategy objectives and strategy's external and internal drivers .......................201

Table 7-9: Primary objectives of environmental strategies in the five generators ...............208

Table 8-1: Simons (1991) types of controls and control systems ...................................213

Table 8-2: Additional types of controls and control systems..........................................221

Table 8-3: Ranking of organisational objectives of MCS, matching with MCS types and groups and environmental strategies ....................................................238

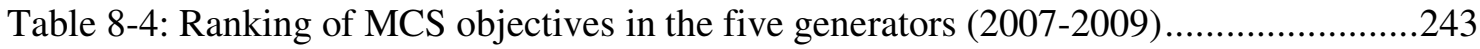




\section{FIGURES}

Figure 2-1: Summary of climate change policy changes from 2002 to 2009 ................ 14

Figure 2-2: Summary of the reforms within NZEI (1987-2009) ............................... 17

Figure 2-3: Market share in the wholesale and electricity markets (2003).................. 18

Figure 2-4: A summary of the relationships between GCCPs, operating environments and generators' environmental strategies and organisational MCS ...........25

Figure 3-1: Summary of findings and gaps from the prior literature........................... 47

Figure 4-1: Inter-relationships between TCE, IT and RBT, and their commonalities and

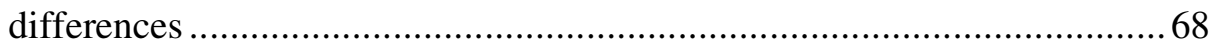

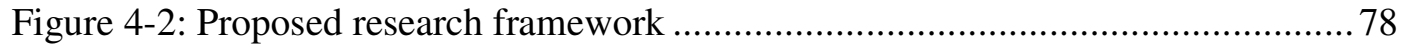

Figure 5-1: Interviewees participating from generators ......................................... 94

Figure 5-2: Break-down of interviewees from external stakeholders by target groups.. 94

Figure 5-3: Breakdown of collected documents by target groups ..............................95

Figure 6-1: Count of evidence of change across the five generators .......................... 160

Figure 7-1: Total GCCP external exposure of the five generators (2000-2009) .......... 174

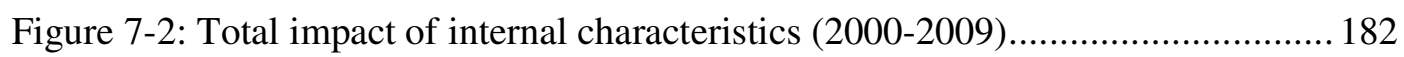

Figure 7-3: Change in total internal capabilities (2000-2009) ................................ 189

Figure 7-4: GCCP-related external exposure, internal capabilities and strategy proactiveness (2000-2009) (P3) .................................................... 193

Figure 7-5: Change in strategy proactiveness (2000-2009) …................................ 195

Figure 7-6: Results framework for theoretical relationships between strategy objectives and external and internal drivers (P3) .............................................. 207

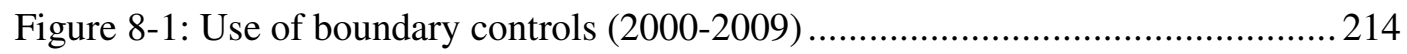

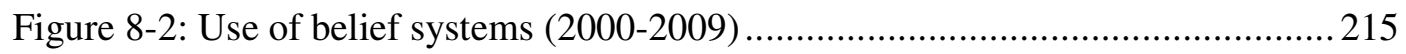

Figure 8-3: Use of diagnostic controls and control systems (2000-2009) ...................217

Figure 8-4: Use of interactive controls and control systems (2000-2009).................220

Figure 8-5: Use of cross-functional controls and coordinating systems (2000-2009).. 222

Figure 8-6: Use of enabling controls and control systems (2000-2009)....................224

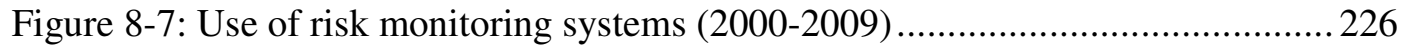

Figure 8-8: Use of legitimising controls and control systems (2000-2009)................ 228

Figure 8-9: Use of the internally-orientated strategy MCS group (2000-2009)........... 230

Figure 8-10: Use of externally-orientated strategy MCS group (2000-2009).............. 231

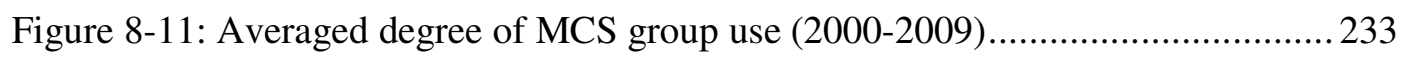

Figure 8-12: Distribution of MCS objectives (2007-2009) .................................... 242

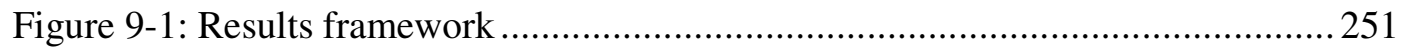




\section{LIST OF ABBREVIATIONS}

\begin{tabular}{|l|l|}
\hline AR & Annual report \\
\hline CCS & Carbon capture and storage \\
\hline CSR & Corporate social responsibility \\
\hline ECNZ & Electricity Corporation of New Zealand \\
\hline EECA & Energy efficiency and conservation authority \\
\hline EMS & Environmental management system \\
\hline ETS & Emissions Trading Scheme \\
\hline GCCPs & Government's climate change policies \\
\hline GHG & Greenhouse Gas \\
\hline GRI & Global reporting initiative \\
\hline HRM & Human resource management \\
\hline IT & Institutional theory \\
\hline LMC & Longitudinal multiple-case study \\
\hline MCS & Management control systems \\
\hline MED & Ministry of Economic Development \\
\hline MfE & Ministry for the Environment \\
\hline Moderated ETS & ETS designed under the National Government \\
\hline NGAs & Negotiated Greenhouse Agreements \\
\hline NZBCSD & New Zealand Business Council for Sustainable Development \\
\hline NZEI & New Zealand Electricity Industry \\
\hline NZES & New Zealand Energy Strategy \\
\hline Original ETS & ETS designed under the Labour Government \\
\hline PRE & Projects to reduce emissions \\
\hline R\&D & Research and Development \\
\hline RBT & Resource-based theory \\
\hline RMA & Resource Management Act \\
\hline SBN & Sustainable Business Network \\
\hline SOE & State-owned enterprise \\
\hline SoS & Security of supply \\
\hline TCE & Transactional cost economics \\
\hline VER & Verified emissions reduction \\
\hline & \\
\hline
\end{tabular}





\section{Chapter 1 - Introduction}

\subsection{Background}

The Government recognises that New Zealand needs to do its share to help the world address the challenges presented by climate change (New Zealand Government, 2007a). On 19 December 2002, the New Zealand Government ratified the Kyoto Protocol and since then has developed and revised different policy packages in order to meet its Kyoto obligations (MfE, 2008). On 29 April 2002, a preference for carbon tax was announced as a key measure of the Government climate change policies (GCCPs). The purpose of a carbon tax was to change behaviour through an imposed tax on carbon emissions generated from the transport and energy sectors. Then, from 2003 to 2004, the Government implemented the Projects to Reduce Emissions (PRE) scheme which grants carbon credits to projects that reduce emissions, i.e. wind farms and landfill projects. However, in 2005, the Government decided that they would no longer proceed with the proposed carbon tax, but did not announce a substitute policy.

In October 2007, the Government announced a new climate change policy package, including an Emissions Trading System (the Original ETS) and supporting sustainability initiatives (MfE, 2008). The fundamental aim of the ETS is to manage climate change through the use of an economic intervention to engage emitters and the community in emission reduction activities. As a result of ETS implementation, there is expected to be an increase in energy and fuel prices due to the added cost of emissions. The resulting price increase will affect all sectors and encourage more efficient and effective use and the adoption of low-emissions technologies. The Original ETS was enacted on 20 September 2008. From October 2008, as a result of the change in Government, the Original ETS was delayed and then reviewed. An ETS Review Bill was drafted and tabled in Parliament in September 2009. This Bill proposed a number of changes to the Original ETS including a price cap of \$25 per carbon credit. This Bill was passed into law on 25 November 2009 (hereafter referred to as to the Moderated ETS)

The introduction of the GCCPs is likely to bring additional volatilities and pressures to bear on New Zealand businesses. As a regulatory economic intervention, a GCCP such as a carbon tax or an ETS, monetarises carbon emissions and internalises them within an organisation's strategic and operational decisions. Therefore, organisations and sectors that have compliance obligations under the GCCPs or are indirectly affected by 
them through carbon-related electricity and fuel price increases, are likely to experience significant cost rises.

Among various sectors in the economy, electricity generators (hereafter referred to as generators) are likely to be those most substantially affected by GCCPs. Since an increase in electricity prices will ultimately affect all sectors, electricity generators have been the target of the various GCCPs since 2002. Further, due to the burning of fossil fuels to generate electricity, thermal generators have a high level of emission intensity. The long useful life of generating assets means that once the choice of a thermal-based technology is made, a generator will incur substantial amounts of total emissions and associated carbon compliance costs under the GCCPs. Carbon costs for electricity generators are also likely to be higher than for other sectors, because generators are among the first to enter the ETS (from 1 July 2010). Hence, they will experience the immediate impacts of the ETS. In contrast to other sectors, generators do not get any financial assistance from the Government (e.g. free carbon credits) to help them mitigate these impacts (New Zealand Government, 2007a).

In addition to the direct financial impacts, the GCCPs have a significant influence on generators' external operating environments. Firstly, the various GCCP changes from 2002 to 2009 are likely to impose significant uncertainty and volatility on the generators. Secondly, a carbon tax or ETS changes the economics of the fuel sources available for generation use, and hence affects generators' investment decisions. Thirdly, the enforcement of an ETS creates a demand for carbon credits, which can be sourced from domestic or international markets. Further, to meet their obligations under the ETS, generators are inevitably exposed to carbon market fluctuations. Lastly, the public debate and discussion around the GCCPs intensifies societal pressures and expectations regarding climate change responsibility (Tomkins, 2009).

Consequently, the GCCPs are likely to impose direct compliance costs on electricity generators as well as expose these organisations to additional external volatilities and pressures. The research problem that arises is, what internal changes have been planned, and implemented within generators to manage organisational performance in the changing GCCP-focused environments where past externalities will be internalised in the future. This study examines changes in the environmental strategies generators adopt to respond to volatilities and pressures associated with the GCCP changes. In doing so, the implications for the organisational management control system (MCS) to enable the effective planning and implementation of these strategic changes can be 
identified. This study is motivated by a number of practical and theoretical reasons, as discussed next.

\subsection{Motivations}

This study is operationally and theoretically important because a search of the existing practice-oriented and academic literatures has found very little research that addresses the research problem outlined above.

\section{Operational motivations}

Practice-oriented literature makes some suggestions regarding the possible strategic responses organisations can adopt to control carbon costs and respond to GCCP-induced volatilities and pressures (PWC, 2008, 2009; Brown, 2009; Tomkins, 2009). An increased emphasis on carbon emission management will be required in internal strategies (such as in production planning and generation investments) as well as in external disclosure and reporting (Tomkins, 2009). Organisations will also need to identify climate change opportunities and formulate sustainability-oriented strategies to seize such opportunities and gain a competitive advantage (Brown, 2009; PWC, 2009).

In a GCCP-focused context, the key challenge for organisations lies in designing and using an appropriate MCS to enable them to implement their strategic responses in an effective and efficient manner. Therefore, organisations will need controls and control systems that measure, monitor and report their organisational carbon emission levels and emissions-reduction initiatives (NZBCSD, 2002; PWC, 2009; Brown, 2009). A carbon accounting system is required to identify potential carbon cost saving opportunities and to achieve compliance with GCCP requirements (NZBCSD, 2002). Appropriate MCS design and use will also allow organisations to monitor external carbon prices and appraise the impacts of price volatility on their operations and compliance costs, based on which appropriate strategic and operational changes can be planned (PWC, 2008).

However, the key limitation with these suggestions from the practice-oriented literature is that they are descriptive and prescriptive, without empirical evidence to support their validity. As a result, little is known about what changes have been implemented within New Zealand organisations, especially generators, to prepare themselves for the planned GCCPs. In particular, the above suggestions regarding MCS are strongly compliancefocused and do not provide sufficient links to the strategic changes generators might make in response to the volatility and pressures associated with the GCCPs. 
Consequently, in examining changes in organisational MCS to support the strategy choices made by generators in managing the organisational impacts imposed by the various GCCPs, this study is motivated by four practical reasons. First, there is a potential to identify the GCCP-related external and internal factors that drive or affect generators' decisions to change their strategies. Second, this study can provide an understanding of how generators have responded and adapted organisationally to the changing external environments associated with the planned GCCPs. Third, it can assess the implications for organisational MCS to enable and support the strategy choices generators make in relation to the GCCPs. An assessment can be made regarding the extent to which generators have changed their MCS to enable and support the pursuit of lower-impact strategies aimed at managing the financial pressures of the GCCPs. Fourth, practical insights can be gained regarding why generators chose and undertook the strategic and MCS changes they did. In this way, the motivations underlying their GCCP-related internal decisions can be identified.

\section{Theoretical motivations}

The theoretical importance of this study stems from a number of research gaps identified in the prior academic literature. Environmental regulation is found to be a significant driver of the adoption of environmental policies and initiatives by organisations (Garrod and Chadwick, 1996; James et al., 1999; Doonan, Lanoie and Laplante, 2005). However, these studies have primarily focused on traditional environmental regulations that target individual industries and sectors rather than the GCCPs which have economy-wide impacts. Furthermore, the traditional environmental regulations specify environmental management standards, while the GCCPs impose direct and wide-ranging economic impacts on organisations through the monetisation and internalisation of carbon emissions. The prior studies also lack a coherent basis to explain the mechanisms by which economic factors influence strategy and MCS choice. Therefore, there is a lack of explanation as to how and why environmental regulation with strong economic impacts such as a carbon tax or an ETS, may lead to a change in environmental strategies and MCS.

Additionally, many studies identify other external and internal drivers relating to the organisational choice of environmental strategies. Externally, economic volatilities (e.g. market competition and regulatory changes) and social pressures (e.g. from different stakeholder groups, peer and field groups, and wider society), are found to motivate organisations to undertake particular environmental strategies (Bowman and Haire, 1975; Greening and Gray, 1994; Sharma, 2000; Brammer and Pavelin, 2004; Bansal, 
2005). Internally, prior studies suggest that factors such as organisational size, technological and financial capabilities, and managerial awareness and commitment, the organisation's prior experience and expertise, and organisational culture, drive organisations to pursue particular environmental strategies (Bansal, 1995; Sharma and Vredenberg, 1998; James et al., 1999; Collins et al., 2007). However, these studies consider these drivers independently from each other, and thus few insights are available regarding the interactions and dynamics between internal and/or external drivers in relation to organisations' environmental strategies.

Prior research also indicates that a number of controls and control systems are required in the implementation of organisations' environmental strategies. These include informational scope and budgetary controls, environmental performance management systems, environmental accounting, and communication and coordination systems (Hutchinson, 1996; Judge and Douglas, 1998; Chenhall, 2003). Additionally, these controls and control systems can be used diagnostically or interactively (Simons, 1991), and employed in internal decision-making, as well as for external disclosure purposes to demonstrate environmental responsibility (Bungay and Goold, 1991; Judge and Douglas, 1998). However, these studies fail to distinguish between different roles played by organisational MCS. Thus, little is known about which controls and control systems are primarily used to control existing environmental strategies, and which MCS components/types assist strategy review and planning.

Therefore, in conducting its empirical enquiry, this study is further motivated by five theoretical reasons. First is the potential to understand the choice and change of environmental strategies under the impacts of the GCCPs. Second, utilising an appropriate theoretical lens, a further explanation can be gained about how environmental regulations with strong economic impacts influence environmental strategies and MCS. Third, insights can be gained regarding the impacts of a longitudinal period of GCCP changes on the external and internal environments in which organisations operate and the inter-relationships between external and internal drivers in driving environmental strategies and MCS responses. Fourth, there is an opportunity to further examine the link between environmental strategy and organisational MCS and the role of MCS in strategy planning. The different types of controls and control systems that serve varying roles in relation to environmental strategies can be examined to determine how they are used to fulfil these roles. Fifth, this study is motivated by a potential to develop a theoretical framework to explain organisations' internal adaptation to external environmental regulatory changes. 


\subsection{Research aim and research question}

Based on the background and practical and theoretical motivations discussed above, it can be argued that the GCCPs will impose direct financial costs and potentially change the external economic and social environments in which generators operate. These costs and external changes are likely to affect environmental strategies and have implications for organisational MCS design and use. Therefore, the aim of this study is to examine the impacts of GCCP changes (from 2002 to 2009) on the environmental strategies and organisational MCS of New Zealand electricity generators. This aim leads to the following research question:

\section{What are the MCS implications arising from strategic choices New Zealand electricity generators make in managing economic volatility and societal pressures resulting from a prolonged period of GCCP change?}

\subsection{Research framework}

To answer the research question, this study adopts a multiple-theory research framework that builds on different but complementary insights from transactional cost economics (TCE), institutional theory (IT) and resource-based theory (RBT). These three theories provide a useful basis to examine the GCCPs' multiple impacts on organisations' environmental strategies and MCS. Transactional cost economics considers the implications of GCCP-related economic factors in the generators' external and internal operating environments on organisational transactional costs, which in turn drive strategy and MCS decisions (Williamson, 1975; Covaleski, Dirsmith, and Samuel, 2003). Institutional theory focuses on the social, political and cultural aspects of organisations' environments and argues that these aspects lead organisations to undertake strategy and MCS change for legitimacy purposes (Meyer and Rowan 1977; Covaleski and Dirsmith, 1983). Conversely, RBT highlights that in order to survive, organisations need to pursue differentiation-oriented environmental strategies by developing and utilising unique internal capabilities and resources, including appropriate MCS, that can generate a competitive advantage (Barney, 1991; Hart, 1995). Although providing different insights regarding strategy and MCS, these three theories have a number of commonalities. Firstly, all of them examine organisational performance, though from different perspectives, i.e. competitiveness, costs, or legitimacy (DiMaggio and Powell, 1983; Williamson, 1991; Barney, 1991). Secondly, they all consider the importance of institutional environments and institutional demands 
and expectations (Williamson, 1991; Covaleski et al., 2003). Thirdly, resources are recognised as an important factor in explaining organisational changes driven by legitimacy, cost control, and/or competitiveness concerns (Meyer, Scott, and Deal, 1983; Williamson, 1999; Barney, 2001).

Therefore, using the three theories simultaneously enables the capturing of a wide range of GCCP-related external and internal factors and their impacts on generators. Furthermore, it is possible to understand the multiple objectives and motivations underlying the environmental strategy and MCS choices made by generators in a GCCP-related context. A multiple-theory framework also allows for theory triangulation through assessing the relative importance of different GCCP-related factors to a generator and investigating how such importance is reflected in its strategy choice and MCS design and use. Consequently, the use of the three theories in conjunction provides richer insights than are potentially achievable by using a single theory. For example, IT enriches TCE by considering the institutional factors in costminimising decisions and thus explaining why in some cases, optimal efficiency is not achievable (Roberts and Greenwood, 1997). Resource-based theory enhances IT and TCE insights by explaining why organisations with particular characteristics and capabilities are seen as more legitimate, or are able to achieve a higher degree of internal efficiency (Barney, 2001). Institutional theory extends RBT by suggesting that competitive advantage needs to be generated within the bounds of what is considered acceptable by the institutional environments (Meyer and Rowan, 1977; Hart, 1995).

Based on the three theories, a multiple-theory research framework is developed that also integrates the external and internal drivers of environmental strategies as identified from the review of the industry-related and academic literature (as briefly outlined in Section 1.2). These external and internal drivers are proposed as the relevant factors that influence or drive the generators' choice of environmental strategies under a GCCPfocused context. Based on TCE, IT and RBT, it is suggested that GCCP-related external and internal drivers affect the generators' costs, competitiveness, and legitimacy and provide an organisational motivation for environmental strategy change. The research framework further indicates that a change in environmental strategies requires appropriate modification in organisational MCS. To measure the scope and timing of environmental strategies, this study uses strategy proactiveness as suggested in prior studies (Russo and Fouts, 1997; Jansson, Nilsson and Rapp, 2000). Additionally, it is argued that generators may not adopt new environmental strategies but simply place more strategic emphasis on them (i.e. more top management attention to particular 
current strategies). For organisational MCS design and use, this study follows Simons' (1991) classification of MCS types. The potential objectives underlying MCS design and use are also identified based on previous studies (Brunsson, 1989; Hart, 1995; Otley, 1999; Scott, 2001).

The multiple-theory framework leads to four propositions:

P1: External GCCP-related economic and institutional drivers impact on generators' competitiveness, costs and legitimacy.

P2: Internal GCCP-related characteristics and capabilities impact on generators' competitiveness, costs and legitimacy.

P3: The impacts of GCCP-related external and internal drivers in turn lead to a change in environmental strategies.

P4: The change in environmental strategies requires corresponding modifications in organisational MCS.

\subsection{Research methodology}

To answer the research question and assess the four propositions, this study adopts a longitudinal multiple-case study approach. This approach involves a 10-year analysis of multiple cases within a single industry, the New Zealand electricity generating sector. Such an approach allows an investigation of a contemporary event, e.g. the GCCPs, within a real-life context (Yin, 2003). Multiple cases are utilised due to the significant diversity in organisational characteristics possessed by the generators. This approach has more limited depth than a single case study. However, the advantage is that it generates cross-case patterns that can be generalised to other organisations, sectors and contexts (Lillis and Mundy, 2005). Further, it provides an understanding of changes and movements in environmental strategies and MCS over time within multiple organisations concurrently.

Using the longitudinal multiple-case study approach, the five major generators in the New Zealand electricity generating sector are examined. These five generators in aggregate make up $91 \%$ of the wholesale market share and $95 \%$ of the retail market share. There are significant differences between them in terms of the major fuel choice of generation (renewable versus thermal-based), generation volumes and carbon emission levels, and ownership type (privately-owned versus state-owned). Due to these different characteristics, the five generators will experience differentiated degrees of 
exposure to the volatilities and pressures associated with the GCCPs, and thus, will undertake varying strategic and MCS responses. Further, to enable the longitudinal analysis, the study period covers 10 years from January 2000 to October 2009, split into sub-periods that match the key GCCP changes.

The main data sources are interviews and documents which relate to the environmental strategies and MCS within the five generators from 2000 to 2009. Triangulation between different data sources and between generators and external organisations is used to ensure the reliability of the data and results and to enrich the potential insights gained from analysis (Miller, Dingwall and Murphy, 2004; Flick, 2009). Collected data are coded using a coding system set up within NVivo8. Then, the data are analysed utilising quantitative and qualitative techniques and matrix data displays (Miles and Huberman, 1994; May, 2001; Silverman, 2006). The use of these different analytic tools allows both longitudinal and cross-sectional insights into changes in environmental strategies and organisational MCS, over time and across generators.

\subsection{Thesis structure}

The rest of the thesis is organised as follows. The next chapter, Chapter 2, provides a background overview of the GCCPs and their potential impacts on generators and highlights the need for these organisations to change their environmental strategies and organisational MCS in response. In Chapter 3, prior literature is reviewed to gain an understanding of previous studies' findings regarding the external and internal drivers of organisations' environmental strategies and the design and use of organisational MCS to implement such strategies. Chapter 4 justifies the multiple-theory framework based on the findings from prior literature and insights from the three theories, TCE, IT, and RBT. The use of a longitudinal multiple-case study approach and methods for data collection and analysis will be discussed in Chapter 5. Chapter 6 presents the case-based evidence of changes in environmental strategies and MCS within the five generators across the five time periods from 2000 to 2009. Chapter 7 analyses this evidence and discusses the results regarding GCCP-related external and internal drivers and their impacts on generators' environmental strategies. Following this, Chapter 8 presents the results relating to changes in MCS design, use and objectives to support the generators' environmental strategies, and deduces the implications for organisational MCS in a GCCP-focused context. Lastly, Chapter 9 discusses the key findings, assesses them in relation to the research framework and the propositions, highlights this study's contributions as well as limitations and provides a conclusion to the thesis. 


\section{Chapter 2 - Government climate change policies and Electricity generators}

\subsection{Introduction}

This chapter provides an overview of the study's operational setting and outlines practical motivations underpinning the research question. Specifically, the chapter highlights the practical importance of studying the implications of various GCCPs over a prolonged period from 2002 to 2009 for generators' organisational MCS. The rest of this chapter is organised into four sections. The next section provides an overview of the GCCPs. Following this, the significance of generators from a GCCP perspective and the potential impacts by the GCCPs on these organisations are discussed. The third section reviews practice-oriented literature to understand possible strategic responses and MCS changes that organisations are recommended to undertake in order to effectively respond to the GCCP impacts. The fourth section summarises the industry background, outlines the practical motivations to this study and presents the research question.

\subsection{The Government's climate change policies}

\subsubsection{Climate change and New Zealand's obligations}

Climate change is widely recognised as a global issue. A recent report of the Intergovernmental Panel on Climate suggests that the high level of past and present Greenhouse Gas (GHG) Emissions will cause substantial climate change for the coming century and beyond (New Zealand Government, 2007b). As a result, the world is projected to experience "rises in temperature, increasing sea levels, frequent extreme weather events and changes in rainfall patterns" (New Zealand Government, 2007a, p.ix).

Over time, many countries have begun to recognise that immediate steps need to be undertaken at global and national levels to reduce the GHG emissions caused by human action (NZIER, 2007; MfE, 2008). The Kyoto Protocol is an international agreement linked to the United Nations Framework Convention on Climate Change with an aim to set binding targets for 37 industrialised countries and the European community to reduce GHG emissions. New Zealand ratified the Kyoto Protocol on 19 December 2002 
and committed itself to reducing its average net GHG emissions over the first commitment period (2008-2012) to below 1990 levels or to take responsibility for the difference by purchasing or generating Kyoto-compliant units (MfE, 2008).

The GHG emissions from the New Zealand economy have grown strongly since 1990, mostly due to growth in the economy (MfE, 2009c). Total gross GHG emissions in 2008 were $22.8 \%$ in excess of the 1990 levels, representing an average of $1.3 \%$ growth per year (MfE, 2009c). Contributing primarily to this growth are the energy and industrial process sectors. The energy sector contributed $45.3 \%$ of total emissions in 2008 and emissions from electricity generation in 2008 increased by $140 \%$ when compared to 1990 levels (MfE, 2010). Without appropriate policy measures, strong growth in emission levels would continue and New Zealand would therefore be unlikely to meet its international obligations (New Zealand Government, 2007b).

\subsubsection{An overview of the history of climate change policies}

The New Zealand Government has developed and revised different climate change policies since 2002 in order to meet its Kyoto obligations. On 29 April 2002, the Government released its 2002 Climate Change Policy Package which had the following significant components (MfE, 2008):

- A carbon tax on energy, industrial, and transport emissions, capped at \$25 per tonne of carbon dioxide equivalent ( $\mathrm{CO} 2 \mathrm{e})$, to be applied to most economic sectors and gas emissions, except for agricultural methane and nitrous oxide emissions.

- Negotiated Greenhouse Agreements (NGAs) for large emitters who face potential changes in their competitiveness compared to producers in countries with less stringent or no climate change policies. The NGAs aimed to help these emitters improve their production efficiency and reduce emissions at a low cost.

- Projects to Reduce Emissions (PRE) under which the Government would grant Kyoto units to the projects that generate additional emissions reductions in the first commitment period.

After this announcement, substantial debate emerged domestically questioning the appropriateness and effectiveness of a carbon tax scheme in meeting New Zealand's Kyoto obligations. This led to the review of climate change policy options in 2005. Following the Review of Climate Change Policies and Next Step report (MfE, 2005a), in December 2005 the Government abandoned the proposed carbon tax but made no announcement as to its replacement (MfE, 2008). Additionally, no further NGAs and 
PREs were available after 2005. Consequently, from January 2006 to September 2007 there was no explicit GCCP. However, a draft of the New Zealand Energy Strategy (NZES) released issued in December 2006 indicated a preference for an ETS and identified emissions reduction and renewable investments as among its key objectives (MED, 2006).

In October 2007, the Labour Government announced its new package of climate change policies, including the Cap-and-Trade Emissions Trading System (ETS), a ten-year ban on new thermal generation (thermal ban), and a target to have $90 \%$ of electricity generated from renewable sources by 2025 (MfE, 2008). This ETS (hereafter referred to as the Original ETS) was to be implemented on a nation-wide basis, across all industries and sectors (New Zealand Government, 2007a). The Climate Change Bill, which outlined the operational mechanisms of the ETS and the thermal ban, was released for public consultation in December 2007 and became respectively the Climate Change Response (Emissions Trading) Amendment Act and Electricity (Renewable Preference) Amendment Act on 25 September 2008. The Electricity (Renewable Preference) Amendment Act (2008) effectively stopped generators from building any new base-load fossil-fuelled thermal plants for ten years, except where an exemption was granted to ensure national security of supply (SoS).

Under the Original ETS legislation, the required entry date of the stationary energy sector into the scheme, including generators, was to be 1 January 2010. The generators were required to take $100 \%$ responsibility for their carbon emissions and, thus, would receive no financial assistance from the Government, as the latter considered that these generators would be able to pass the cost of carbon fully to customers. In contrast, large industrial organisations that compete with overseas producers were to be assisted by the Government for up to $90 \%$ of their emissions so as to partially mitigate the impact of electricity and fuel cost increases due to the ETS.

In October 2008, the National Party won the general election and formed a new government. As a result of an agreement with the ACT Party, the National Government set up an ETS Review Select Committee to examine the Original ETS (New Zealand Government, 2008). The National Government also removed the thermal ban in December 2008 as part of this agreement (Brownlee, 2008). The report from the ETS review committee was released on 31 August 2009. Based on this report's recommendations, an ETS Review Bill (its full name being Climate Change Response (Moderated Emissions Trading) Amendment Bill) was drafted and tabled in Parliament on 24 September 2009 (MfE, 2009b). For the transitional period from 1 July 2010 to 31 
December 2012, this revised ETS (hereafter referred to as the Moderated ETS) puts a NZ $\$ 25$ cap on carbon prices and requires participants in the stationary energy and liquid fossil fuel sectors to surrender only one carbon credit for every two tonnes of emissions (50\% obligation). The ETS Review Bill also proposed a new entry date for the stationary energy sector into the Moderated ETS from 1 July 2010. This Bill was passed into law on 25 November 2009 (NZPA, 2009).

Consequently, between 2002 and 2009, the GCCPs underwent several changes. From April 2002 to December 2005, the main GCCPs were the carbon tax plan and the PRE scheme. From January 2006 to September 2007, the carbon tax was abandoned, the PRE scheme discontinued and an ETS was signalled. From October 2007 to September 2008, the announcement of the Original ETS and the passage of its legislation were the key GCCP focus. However, from October 2008 to October 2009, there was a change of government which led to a select committee review of the Original ETS and substantial legislative changes being proposed under the ETS Review Bill. These changes are summarised in Figure 2-1.

Figure 2-1: Summary of climate change policy changes from 2002 to 2009

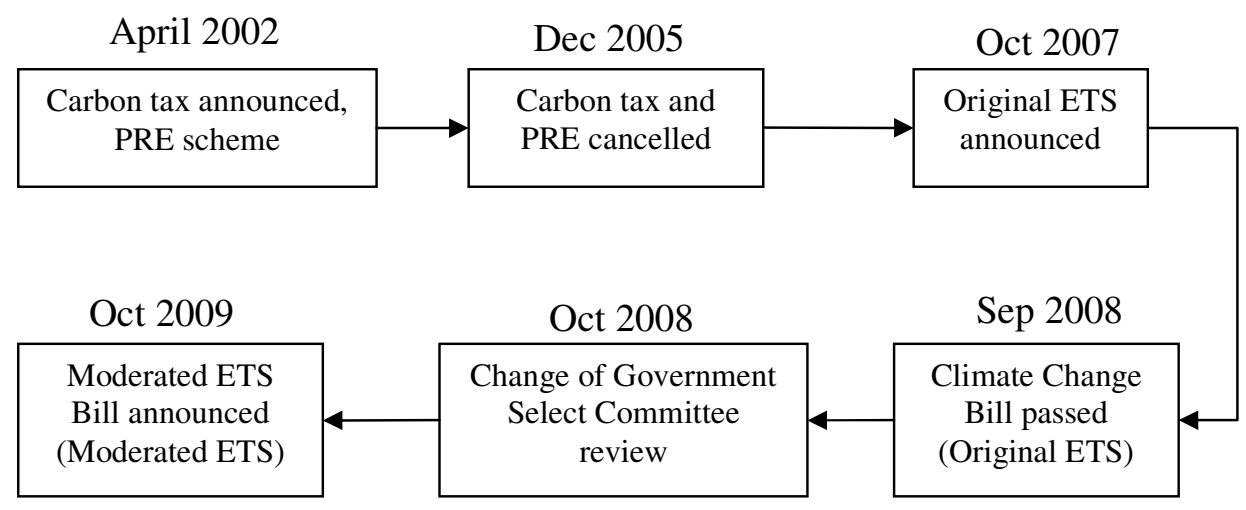

The GCCPs, such as the carbon tax and an ETS, are economic instruments through which the Government and their regulators re-allocate resources in the economy among different sectors to achieve particular environmental, economic and social objectives (Helm, 2005). In the case of the GCCPs, they are designed to ensure that businesses "start taking the environment into account in the economic choices" they make and thus combine economic interests with environmental ones (Hodgson, 2005, p.1). Accordingly, carbon pricing affects the prices of goods and services and makes energyintensive technologies more expensive, while clean technologies become cheaper and more affordable. Additionally, carbon pricing affect both demand and supply sides of the market, thus encouraging climate-friendly investments and behaviour, not only by businesses and other organisations, but also by consumers (Helm, 2005; Parker, 2008a). 
Furthermore, integrating a price for emissions into the economy enables New Zealand to transit smoothly into a future carbon-constrained world where limits are placed on carbon emissions and rights to emit are traded (Hodgson, 2005; Parker, 2008a). Therefore, the GCCPs are not purely environmentally-driven, but are also motivated by the economic management necessary to position the New Zealand economy favourably in the future.

This study chooses to focus upon the impacts of these GCCPs on the generators. The next section explains the reasons for this choice and provides a background to the NZEI.

\subsection{Electricity generators and the potential impacts of the GCCPs}

\subsubsection{The significance of the electricity generating sector from a GCCP perspective}

Though the GCCPs and the associated carbon pricing mechanisms affect all sectors, their impacts on the electricity generators are different and likely to be more significant than in many other sectors. Electricity is essential to the functioning of the economy and society and thus ensuring a secure, affordable and environmentally responsible supply of electricity is very important (MED, 2006). Further, climate change has become a cornerstone issue in the development of the national energy strategy (Clark, 2007; Parker, 2008b). Therefore, how to accelerate the uptake of renewable technologies, and achieve substantial carbon emissions reductions from electricity generation, is one of the key objectives of New Zealand's GCCPs (Parker, 2008b). Consequently, the electricity generating sector is the direct target of many of the GCCPs. Accordingly, it receives more attention and emphasis than most other sectors within the scope of GCCP design.

Further, given the asset-focused and technological nature of electricity generation, generators are likely to be those most significantly affected by any GCCP. First, electricity generation is one of the most emission-intensive sectors in the economy because a high level of carbon emissions from the combustion of fossil fuels is an inevitable product of the generation process (MED, 2007b). Second, electricity generation entails large capital investments with the lives of generating plants spanning decades and requiring a long pay-back period. If a generator chooses to use a thermalbased technology for one of its plants, there is generally a high level of stability in the amount of fuel used over the plant's useful life with little variation in the level of carbon emissions discharged (MfE, 2005a). Third, generators will be among the first to enter 
the ETS (from 1 July 2010) ${ }^{1}$ and accordingly, will incur ETS-related emissions liabilities earlier than many other sectors. These generators receive no financial assistance from the Government, i.e. granting of free carbon credits to help offset carbon costs (New Zealand Government, 2007a). However, under the Moderated ETS, their obligations will be halved until the end of 2012 .

The above factors suggest that not only is the electricity generating sector the focus of many of the GCCPs, it is also likely to incur a higher degree of carbon costs than many other sectors. Such costs have significant implications on organisational profitability and potentially survival in the short and long term. An examination into how generators respond and adapt organisationally to the changing GCCPs potentially provides critical understanding regarding the effectiveness of the GCCPs in triggering businesses' strategic shift towards the adoption of more climate-friendly technologies and processes. Such a strategy shift, in turn, requires appropriate modifications in organisational MCS design and use in order to enable efficient and effective strategy implementation. An investigation of strategy and MCS change also allows insights into how organisations exposed to the impacts of the GCCPs can manage their organisational performance. For these reasons, this study chooses to examine generators and their strategy responses to the GCCPs and the implications for organisational MCS. An overview of the structure and history of the NZEI is provided in the next section.

\subsubsection{History and Structure of the NZEI (1987-2003)}

The New Zealand electricity industry was, until 1987, completely publicly owned and regulated. From 1987 a series of reforms were undertaken to create competitive markets for electricity generation and retailing, while maintaining natural monopolies in transmission and distribution. Figure 2-2 below presents the timeline of the reforms from 1987 to 2003 in NZEI. These reforms led to changes in the structure of the NZEI. This new structure, as described below, remains relevant throughout the period investigated by this study (2000-2009).

\footnotetext{
${ }^{1}$ The forestry sector entered the ETS from 1 January 2008, followed by the transport and energy sectors both of which will enter the scheme from 1 July 2010.
} 


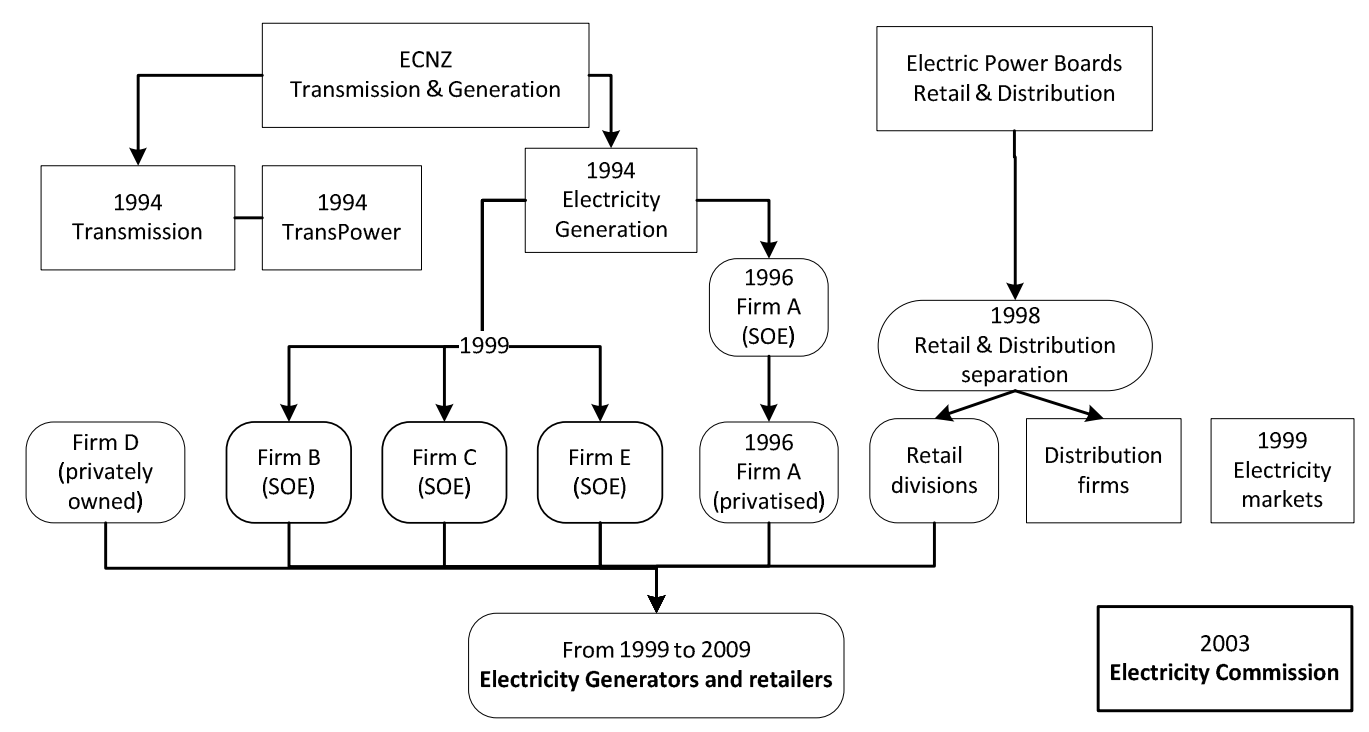

In 1987, the Electricity Corporation of New Zealand (ECNZ) was established as a state owned enterprise (SOE) to operate as a commercial, profit-making organisation and was the sole provider of electricity generation, transmission and retail. Electricity was distributed through local electricity supply authorities, which were later corporatised into line companies. A subsidiary of ECNZ, TransPower, was specifically responsible for electricity transmission. In 1994, TransPower was separated from ECNZ and became an independent SOE. In 1996, ECNZ was further separated into two entities: ECNZ and Firm A (a SOE), and a wholesale market was established. In 1999, ECNZ was further split into three SOEs (Firms B, C, and E) and Firm A was privatised. As a result, many of the generation assets currently owned by these four generators $(\mathrm{A}, \mathrm{B}, \mathrm{C}$ and E) were inherited from the ECNZ.

Coinciding with the break-up of the ECNZ, the Electricity Industry Reform Act 1998 required electricity companies to separate ownership of their line and supply businesses. Taking this opportunity, some generators (Firms A, B, C, D and E) acquired these retail businesses and became vertically integrated. By 2003, all these generators had completed their acquisition activities and became the five biggest electricity generators and retailers in the industry (Murray and Stevenson, 2004; Hogan and Meade, 2007). By 2003, aggregately, these five generators comprised $91 \%$ of national electricity generation, and $95 \%$ of the retailing market share (Figure 2-3). 
Wholesale market share

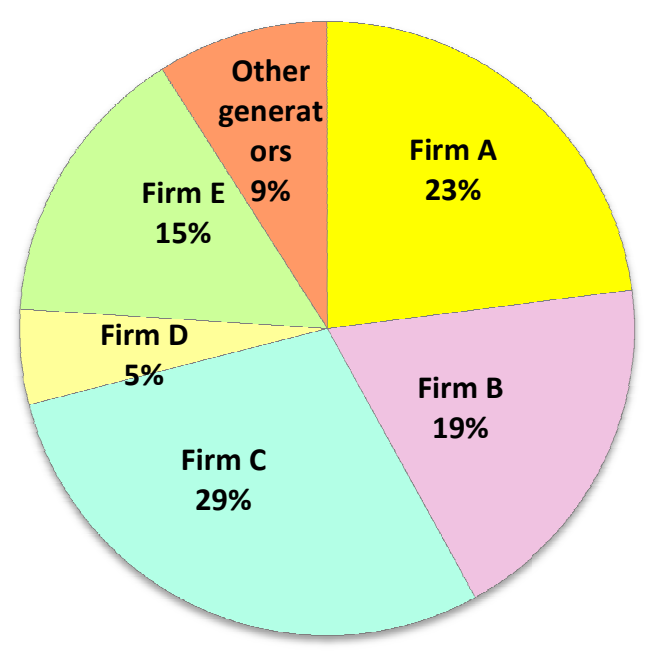

\section{Retail market share}

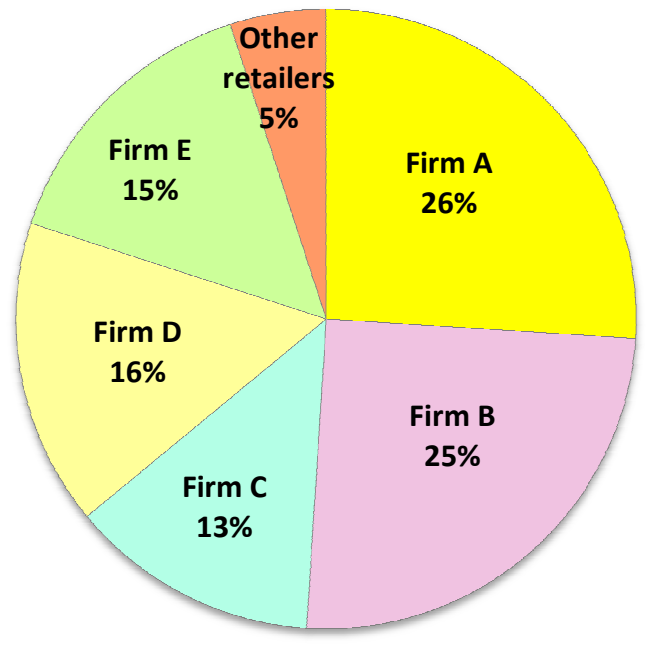

Source: Murray and Stevenson, 2004

It is these five key generators (and retailers) that are the focus of this study. To maintain the confidentiality of the interview data relating to these generators, they will, hereinafter, be referred to as Firms A, B, C, D and E. These firms are the significant 'players' in the generation and retailing markets and are also those responsible for the bulk of the carbon emissions discharged from the electricity generating sector. The generators also differ significantly from each other in terms of ownership structure and asset base. Three generators are state-owned and two are privately-owned and publiclylisted. Additionally, two generators are thermal-based (with the majority of generation volume coming from fossil fuels) and the other three are renewable-based. More details on the internal characteristics of these generators are provided in Chapter 5.

As a result of the NZEI reforms, since 1999, two markets are operational, the wholesale and the retail electricity markets. The wholesale electricity market is the market where generators compete to sell their generated electricity to electricity retailers and other purchasers such as major commercial and large industrial users. The retail market is where retailers compete to sell the electricity purchased from the wholesale market and from embedded generators ${ }^{2}$ to residential customers and small-scale industrial and commercial users who do not participate directly in the wholesale market (EC, 2006). In 2003, the Electricity Commission was set up by the Government to oversee the industry

\footnotetext{
${ }^{2}$ Embedded generators are generators who are connected directly to distribution lines, rather than the national grid.
} 
after the industry failed to regulate itself (Evans and Meade, 2005). The role of the Electricity Commission is to monitor the electricity markets to ensure market participants comply with market rules and that the markets operate efficiently on a dayto-day and long-term basis (EC, 2006).

Electricity is produced at 40 generation plants in New Zealand, using mainly coal, gas, and hydro and to a lesser extent, wind and geothermal energy as fuels. Electricity generated is then transferred to the transmission network owned and operated by TransPower. The national grid transmits electricity from generation plants to the points of distribution (grid exit points), where it is reduced to a lower voltage and transmitted through distribution networks to consumers (EC, 2006). Distribution networks are owned and operated by around 28 line businesses throughout New Zealand. They largely sell their distribution services to retailers who in turn manage electricity supply contracts with end users or sell directly to large commercial and industrial consumers (EC, 2006). These 28 businesses are operated as trusts and, like TransPower, distribute power on a monopoly basis. Due to their monopolistic nature and the low carbon emission levels of transmission and distribution operations, both TransPower and the line businesses are not examined in this study.

Consequently, between 1987 and 2003, NZEI has undergone significant structural and regulatory reforms. These reforms have resulted in an electricity industry characterised by generators having inherited assets from the ECNZ. The generators have high market concentration and are vertically integrated through both the wholesale and the retail markets. Against this background, the GCCPs were proposed. As discussed in Section 2.3.1, many of the GCCPs were designed to target the electricity generating sector directly. Thus although some of the GCCPs (e.g. the carbon tax and the ETS) were not enforced during the period of study (2002-2009), they still potentially affect generators as strategic decisions needed to be made as to how they were going to respond to planned regulatory/policy changes. The next section reviews practice-oriented literature to understand the potential impacts of the GCCPs on the electricity generators.

\subsubsection{Potential impacts of the GCCPs on generators' operating environments (2002-2009)}

The GCCPs are likely to cause significant changes within generators' internal and external operating environments. The impacts of GCCPs on the generators' external environments include: uncertainty related to planned policy and regulatory change, changing fuel economics, carbon market fluctuations, and changes in societal pressures. Internally, the GCCPs impact on the generators' emission profile through the direct 
imposition of carbon emission and compliance costs. These GCCP impacts apply to the 2002-2009 period, during which different GCCPs were being developed and modified. The implications of the GCCPs on the generators in the later period, from 2010, are outside the scope of this study and thus will not be examined. They, nevertheless, represent an interesting area for future research.

Firstly, from an external environment perspective, the changes in GCCPs over a prolonged period potentially result in substantial policy and regulatory uncertainty for the generators. The uncertainty relates to policy shifts from a proposal for a carbon tax to the Original ETS and then to the Moderated ETS. Summaries of the submissions to the Original ETS Bill by MED (2007) suggest that these policy shifts have led to fears of investments being undermined and thus could lead electricity generators to delay investment, especially in renewable energy. Additionally, generators are exposed to regulatory ambiguity relating to each GCCP. With a carbon tax, regulatory ambiguity relates primarily to the level of the tax to be imposed by the Government (Helm, 2005; Kerr, 2007). Conversely, an ETS increases the vulnerability of generators in relation to issues such as the entry point of the electricity sector into the ETS, the requirements and standards for monitoring, verifying and reporting of emissions and carbon credits, and whether there will be a cap on carbon price (PWC, 2008, 2009). However, such regulatory ambiguity/vulnerability only applies to thermal generators, because renewable generators do not emit carbon emissions from their production activities, and thus are not compliance entities under the GCCPs.

Secondly, the GCCPs are likely to change the relative economics between alternative fuels for electricity generation (MED, 2007). As discussed above, many GCCPs are designed directly to encourage renewable investments. Similarly, modelling conducted by the Government suggests that pricing emissions will increase wholesale prices to the extent that they incentivise significant volumes of cost-competitive renewable generation (Parker, 2008a).

Thirdly, under both the Original and Moderated ETS, electricity generators are exposed to carbon market fluctuations. Although there is a lack of certainty about future supply and demand in the international carbon markets, there is a high possibility that the price of carbon will rise and there will be substantial volatility in the market (MfE, 2005a; NZIER, 2007). Furthermore, there are project risks, contract risks and country risks associated with different types of carbon credits available on the international carbon markets (MfE, 2005a). Such variability and fluctuations in carbon markets impacts on compliance costs and influences return on investment (Kerr, 2007; Tyagi, 2009). 
However, a preliminary analysis of the generators' ETS obligations indicates that the level of exposure to the carbon market depends on whether a generator is a net seller or a net buyer of carbon credits. Most generators have some carbon credits awarded to their renewable projects under the PRE scheme in the 2003-2005 periods (MfE, 2009a). By deducting these carbon credits from their ETS obligations (under the Moderated ETS), renewable generators will be net sellers of carbon credits while thermal generators will be net buyers. As net sellers, renewable generators will gain from an increase in carbon prices. In contrast, as net buyers, thermal generators are likely to suffer a profitability loss when confronted with such a price increase.

Fourthly, the planned GCCPs affect, and were affected by, changes in societal perceptions and pressures. From 2004 to 2005, a number of public campaigns were launched by the Government to build awareness of the effects of climate change and what New Zealanders can do to reduce GHG emissions (MfE, 2005b). Societal awareness of climate change has increased as a result. Moxie's (2007) survey found that overall the concern for climate change has grown $22 \%$ from 2005 to 2007 . This concern was argued to correspond to the growing amount of media attention on the issue (Moxie, 2007). Similarly, a national online survey conducted by ShapeNZ (2009) revealed that a majority of New Zealanders believe that climate change is a problem and want the country to respond proactively to climate change. New Zealand society also generally believes that polluters should pay for their own emissions costs and supports an ETS as an economic instrument to make polluters pay (ShapeNZ, 2009). The increased awareness of climate change in turn has led to an increased pressure on generators, especially thermal ones, to take action and mitigate their carbon emissions (Greenpeace NZ, 2007).

From an internal environment perspective, the planned GCCPs impose significant carbon and compliance costs on the generators. As discussed in Section 2.3.1, generators are likely to incur higher carbon costs than other organisations, under both a carbon tax and ETS, because electricity generation is an energy-intensive activity and requires long-term investment. Furthermore, carbon costs vary among the electricity generators due to differing emission profiles. IPENZ (2010) argues that the emission intensity of existing generation plants (i.e. amount of carbon emissions per unit of production) is dependent on the fuel type and the thermal efficiency of a particular generation technology. Hence, generators with higher emission intensity have higher total emissions and are thus exposed to higher carbon costs under the GCCPs (Emissions Trading Group, 2007). 
The introduction of a GCCP will also increase compliance costs for electricity generators, as they will need to comply with emissions-related monitoring and reporting obligations under a carbon tax or an ETS (PWC, 2008, 2009). This is likely to cause additional costs, given that organisations have rarely reported such information before (Milne, Owen and Tilt, 2001; Milne, Tregidga and Walton, 2003). Compliance costs under a carbon tax are likely to be lower because organisations only report their emissions and emissions liabilities. Whereas, under an ETS, they also have to buy and record purchases of carbon credits, and are exposed to the volatility and complexity of the carbon markets (PWC, 2008).

Overall, this section argues that changes in the GCCPs from 2002 to 2009 are likely to have resulted in significant changes in the generators' external and internal operating environments. To ensure survival, generators need to adapt organisationally to these changing operating environments. Specifically, they would need to make strategic responses and corresponding MCS changes to effectively manage their emission profile and reduce their organisation's exposure to the external volatilities and pressures associated with the GCCPs. The next section summarises suggestions from practiceoriented literature on the required changes in organisational strategies and MCS to prepare for the GCCPs.

\subsection{Potential organisational strategic and MCS responses to the}

\section{GCCPs}

Practice-oriented literature makes suggestions as to the possible strategic and MCS responses organisations can adopt in response to these GCCP-induced volatilities and pressures (NZBCSD, 2002; PWC, 2008, 2009; Brown, 2009; Tomkins, 2009). From a strategy perspective, NZBCSD (2002) presents case studies of how six New Zealand companies have formulated and implemented a climate change strategy in response to the Government's Climate Change Policy Package announced in 2002. They argue that this strategy has helped these organisations to benefit in a number of areas including: provision of knowledge and services in renewable energy and carbon trading; energy efficiency and improved resource usage; new climate-friendly technology; and practices and access to new energy products and markets. Further, Brown (2009) and PWC (2009) recommend that organisations formulate a strategy to capture climate change opportunities and gain market competitiveness. Adopting sustainability strategies is also seen to be important in an environmentally-driven mode of business development and provide organisational flexibility to respond to challenges and opportunities presented by a carbon-constrained world (Jansson, Nilsson and Rapp 2000; Brown 2009). Deloitte 
(2007) suggests that companies be creative within their strategy formulation in response to the challenges posed by climate change. They propose that companies can build internal capabilities around emerging carbon trading markets, which not only helps them understand and manage climate change threats, but also can be leveraged and turned into a competitive advantage (Deloitte, 2007).

From a MCS viewpoint, compliance systems need to be put in place to ensure that organisations record, report, and surrender carbon credits on time and accurately to avoid expensive penalties from violating the GCCP requirements (Hunt and Auster, 1990; PWC, 2008, 2009). Further, organisations need their MCS to monitor their emissions profile, and analyse and understand their carbon-related exposure (NZBCSD, 2002; PWC, 2009; Brown, 2009). Appropriate MCS design and use will also enable organisations to monitor external carbon prices and appraise the impacts of price volatility on their operations and compliance costs, based on which emissions reductions initiatives can be identified and implemented (PWC, 2008). Through these MCS changes, organisations can monitor and manage their GCCP-related carbon and compliance costs.

Appropriate MCS design and use can also help organisations maintain their reputation and effectively respond to expectations and pressures by external and internal stakeholders. Hunt and Auster (1990) argue that environmental problems can ruin image and strain relationships with stakeholders and suggest that such problems can be mitigated and prevented by establishing environmental management programmes and systems. The NZBCSD (2002) argues that accurate carbon accounting is important because it enables organisations to identify carbon or cost saving opportunities, demonstrate compliance with GCCP regulations and participate in carbon trading markets. Furthermore, Tomkins (2009) suggests that stakeholders are increasingly requesting companies to provide information regarding their carbon footprint. To ensure accurate reporting of such information, and avoid severe fines and damages to organisational reputation, Tomkins (2009) insists that organisations put in place appropriate controls and control systems to enable comprehensive understanding, measuring and reporting of the organisational carbon footprint.

The practice-oriented literature above prescribes how environment-driven strategies and appropriate MCS can help organisations reduce compliance costs, maintain their reputation and respond to societal pressures. However, no empirical evidence has been provided that examines the extent such strategies and MCS have been implemented by generators in managing the impacts of the GCCPs and preparing organisationally for a 
carbon-constrained world. This literature is also strongly focused on MCS for compliance purposes and lacks insights into the implications for organisational MCS to support and enable the strategic decisions made by generators in a GCCP context. Therefore, there is a need to investigate strategy and MCS changes within generators to understand how they have responded and plan to respond to the economic volatility and societal pressures imposed by the GCCPs.

\subsection{Practical motivations to this study and the Research Question}

The above sections have provided an overview of the changes in the GCCPs from 2002 to 2009. It was emphasised that the electricity generating sector is critical to the achievement of GCCPs and this sector is, and will be, more significantly affected by the GCCPs than most other sectors in the economy. In addition to experiencing a prolonged period of GCCP changes, generators have been exposed to a number of energy-specific Government policies with a climate change objective. These GCCPs are likely to bring about volatilities in the generators' external and internal operating environments. Externally, generators are likely to be exposed to policy and regulatory change, changing fuel economics, as well as carbon market fluctuations and increased societal pressures. Internally, generators will incur significant carbon and compliance costs depending on their organisational emission profile. In order to survive, generators need to change their environmental strategies and associated MCS to properly manage these GCCP-related economic volatility and societal pressures and reduce their impacts on organisational performance.

The potential relationships between the planned GCCPs, the volatilities and changes in the external and internal environments, environmental strategies and organisational MCS of New Zealand electricity generators is illustrated in Figure 2-4, which summarises the arguments presented in this chapter. 
Figure 2-4: A summary of the relationships between GCCPs, operating environments and generators' environmental strategies and organisational MCS

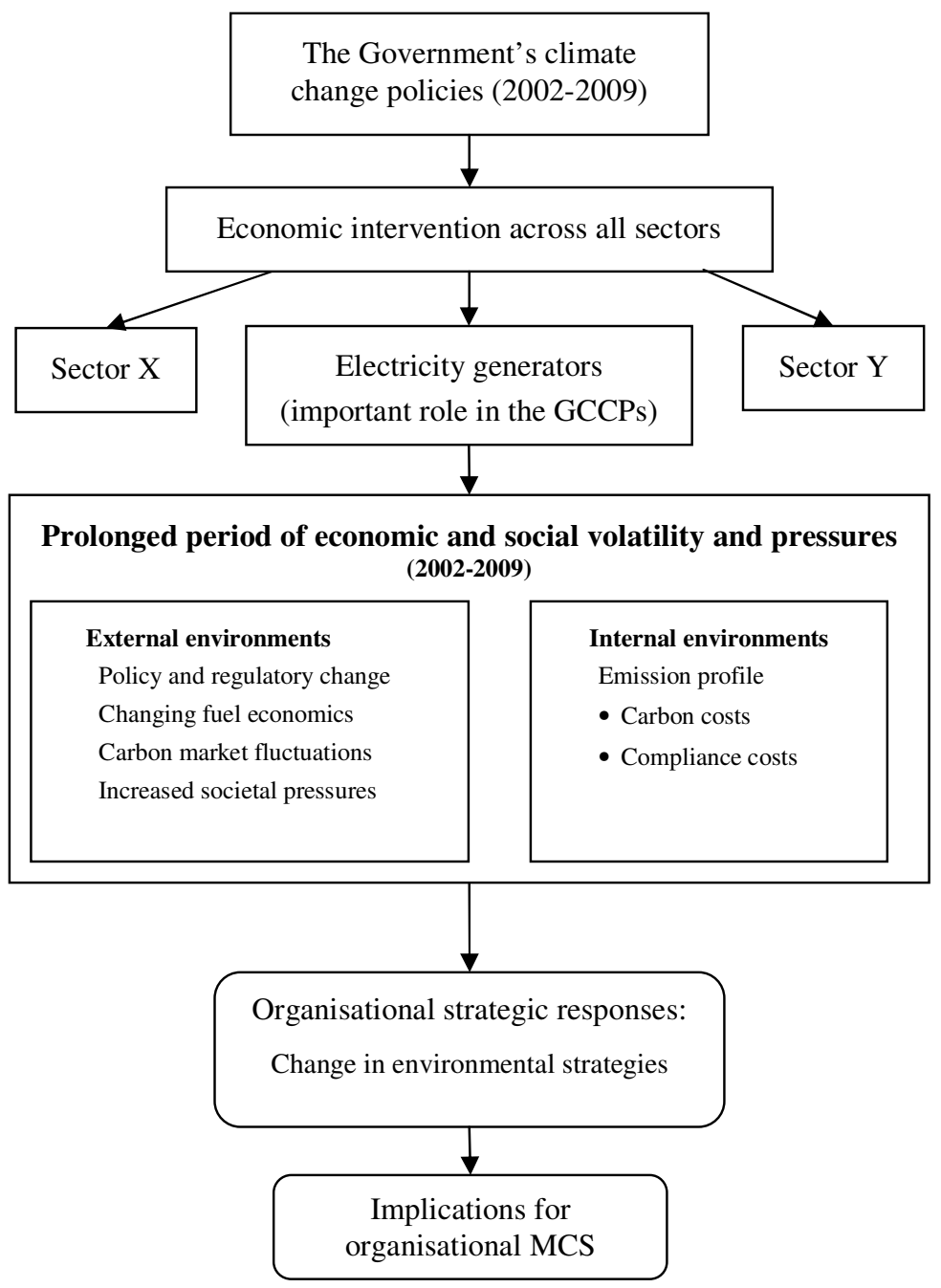

Note: Arrows do not indicate causal relationships

Empirically examining the relationships between the GCCPs, organisational strategies and MCS as illustrated in Figure 2-4 is important for a number of practical reasons. Firstly, there is a need to identify the GCCP-related factors in the generators' external and internal operating environments that drive generators' decisions to change their environmental strategies. In doing so, insights can be generated regarding the role played by not only external drivers but also internal characteristics such as asset and technology base and emission profile in driving the strategy choices and MCS changes generators make in the GCCP-focused context. These insights help practitioners and managers understand and evaluate the sources and the impacts of various volatilities and pressures imposed by the GCCPs on their organisation.

Secondly, this study is motivated by a potential to understand how the generators have responded, and adapted organisationally to the changing operating environments 
associated with the planned GCCPs. This understanding gives practitioners, managers and related stakeholders an insight into what strategy choices organisations can make in order to survive in the changing economic and societal environments associated with the GCCPs.

Thirdly, such an examination helps to assess the implications for organisational MCS so that it enables and supports the strategy choices generators make in relation to the GCCPs. An understanding of these MCS implications is important to accountants, managers and consultants in ensuring compliance with GCCP obligation requirements and preparing their organisations for a carbon-constrained economy. Furthermore, an assessment can be made regarding the extent that generators have changed their MCS to facilitate the uptake of climate-friendly and sustainable strategies. Based on this assessment, regulators and policy makers can evaluate the relative success of the GCCPs in achieving the objectives for which they were originally designed, i.e. more renewable investments and emissions reductions.

Fourthly, practical insights can be gained into why generators made the strategic and MCS changes they did. Accordingly, the objectives of GCCP-related strategy and MCS changes can be identified. Furthermore, the relative importance of these objectives for different generators can be ascertained in terms of whether the strategy and MCS changes are primarily made to reduce carbon and compliance costs, to maintain reputation and respond to stakeholders' pressures, or to enhance market competitiveness. Hence, practitioners and policy-makers can determine whether organisations' internal adaptations are driven by direct financial impacts of the GCCPs or related reputational and competitive concerns.

Based on the practice-oriented literature reviewed in this chapter and these practical motivations, the following research question is formulated:

\section{What are the MCS implications arising from strategy choices New Zealand electricity generators make in managing economic and societal volatility and pressures resulting from a prolonged period of GCCP change?}

To address this research question, in the next chapter, Chapter 3, the relevant literature in environmental management, strategies and MCS is reviewed. This review aims to gain an overview of the findings of prior studies regarding the possible environmental strategies and MCS adjustments that organisations make to respond to environmental issues and regulations. In doing so, external and internal drivers of such strategies and 
MCS adaptations can also be identified. Further, it enables the development of the research framework in Chapter 4. 


\section{Chapter 3: Environmental strategies and MCS}

\subsection{Introduction}

Chapter 2 suggests the GCCPs, with their accompanying legislations and regulations, are likely to affect the generators' external and internal operating environments and have implications for their environmental strategies and MCS. Based on this, a research question was presented that examines the implications for organisational MCS arising from the strategy choices generators make in response to the volatilities and pressures associated with GCCP changes. This chapter reviews the literature to determine what prior academic studies have found regarding the environmental strategies and MCS that organisations can adopt in managing their environment-related organisational performance. This chapter is separated into six sections. The next two sections review research related to the external and internal determinants of environmental strategies and MCS. The following section assesses prior literature to understand the types of environmental strategies that organisations can adopt to respond to environmental issues and regulations. Next, previous studies are examined to identify the design and use of the MCS to support the organisational environmental strategies and the objectives underpinning any environmental strategy-driven change to the MCS. Following this, an overall evaluation of the literature is presented, which highlights the existing gaps and outlines how this study is positioned to address some of these. Lastly, a summary of the chapter is provided.

\subsection{External drivers of environmental strategies and MCS}

\subsubsection{Environmental regulation}

Environmental regulation is one of the most important drivers of organisations' environmental strategy as environmental regulation imposes significant compliance costs on those organisations with high environmental-impacting operations (Garrod and Chadwick, 1996). An intention of this regulation, and the compliance costs it imposes, is to provide an incentive for organisations to adopt more environment-friendly activities and technologies (James et al., 1999; Doonan et al., 2005). However, most of the studies that examine environmental regulation have used an institutional framework. A survey by Garrod and Chadwick (1996) suggests that a shift to environmental concern by organisations is more likely in a regulatory context than in a voluntary one. 
Clemens (1997) argues that environmental issues are considered more important and more institutionalised in those industries with extensive environmental regulation. In two surveys of UK top 400 companies, James et al. (1999) find evidence that legal requirements are the most significant driver of environmental policy formulation across sectors. Similarly, Doonan et al. (2005) and Berrone et al. (2007) suggest that government regulation presents the most significant factor in triggering and promoting organisations' environmental innovation.

The introduction of an environmental legislation or regulation enhances the visibility and urgency of environmental issues. When these issues become visible, they can lead to an outcry from a wide range of stakeholders and put the social legitimacy of organisations under threat. This, in turn, results in a need for organisations to respond promptly and make internal changes (Sobel, 1984). Empirically, Holmes (1976) indicates that managers view the seriousness of social issues as one of the key reasons for organisations to respond to social demands. Dutton and Ducan (1987) argue that organisational response to changing environmental conditions, such as the introduction of an environmental regulation, needs to be based on an assessment of the urgency of these conditions and the organisation's capability to take action. Van Wassenhove and Corbett (1991) suggest that it is possible for a specific organisation to follow a mix of response patterns to different issues depending on their visibility.

The accounting literature also suggests that regulatory pressures are among the most significant external factors that induce similar accounting responses among organisations (DiMaggio and Powell, 1983; Ribeiro and Scapens, 2006). Meyer and Rowan (1977) argue that organisational structures and formal systems increasingly reflect rules institutionalised and legitimated by the state. In some cases, organisational adoption of particular MCS is a direct response to a government mandate, such as the implementation of a pollution control system to conform to environmental regulation (DiMaggio and Powell, 1983). Consequently, environmental regulation produces direct and indirect coercive pressures on organisations to adopt particular controls and control systems that are considered legitimate by the government.

Environmental regulatory change is likely to bring about significant alterations in the economic and societal environments in which organisations operate. Therefore, the introduction of an environmental regulation to deal with climate change (GCCPs), similarly, can change the parameters that define the economic and societal environments (Greenwood and Hinings, 1996; Goodrick and Salancik, 1996). In turn, this can result in a need to make changes to organisational strategy and MCS. The next section reviews 
prior literature to gain an insight into the impacts of other external economic factors on environmental strategy and MCS.

\subsubsection{External economic volatility}

The literature suggests that market-based economic uncertainty/volatility associated with environmental issues can influence the adoption of environmental strategies. Bowman and Haire (1975) suggest that corporate social responsibility (CSR) and sustainability reporting are environmental/social strategies that help organisations to deal with, and reduce external uncertainty. These strategies provide a mechanism through which the organisations can cope with different, and even conflicting, demands from the external environments and, thus, it becomes "an appropriate posture in dealing with a multi-vectored, changing environment" (Bowman and Haire, 1975, p.54). In a similar vein, Azzone and Bertelè (1994) highlight that a proactive environmental strategy enables organisations to quickly adapt to changing environmental standards and regulations by providing flexibility and adaptability and an opportunity to gain green competitiveness.

The prior literature provides empirical evidence of the impact of these external economic volatilities on organisational MCS. Hostility from intense competition is found to be associated with a strong focus on budgets, sophisticated production, and statistical controls (Khandwalla, 1972; review in Chenhall, 2003). In contrast, environmental complexity (related to suppliers and Government) is found to be associated with less focus on budgetary controls (Merchant, 1984). Additionally, prior MCS literature suggests strongly that external uncertainty has a significant impact on organisational MCS (review in Chenhall, 2003). Accordingly, organisations that operate in uncertain environments will adopt a broader-scope MCS and use externally-focused and non-financial MCS information more extensively. Some studies found a combination of tight controls and flexible, interactive controls in conditions of high external uncertainty (Ezzamel, 1990; Merchant, 1990). Simons (1991) argues that formal control systems, such as budgets, can be used interactively in conditions of high uncertainty to encourage new strategic initiatives. Using a different lens, Chapman (1998) suggests uncertainty requires frequent interaction between accountants and other departments/business units to cope with the changing conditions. However, there is little available empirical insight into how external changes associated with environmental regulation, such as the GCCPs, influence MCS design and use. Similarly, the literature has been silent on the impacts of market dynamism (i.e. the speed of changes in external 
market conditions and the availability of opportunities) on organisational MCS (Chenhall, 2003).

Economic volatilities are not the only external drivers of environmental strategies and MCS. Societal pressures also result in environmental strategy change, as discussed in the next section.

\subsubsection{External societal pressures}

External societal pressures pertain to the demands and expectations exerted by external entities (e.g. the Government, professional bodies, and industry groups) on organisations which lead them to make changes to their environmental strategies and associated MCS. These pressures, as examined in the literature, can be grouped into three categories: industry-related factors, stakeholder pressures, and peer and field pressures. Each category is discussed next.

\section{a. Industry-related societal pressures}

Institutional theory has been utilised by many management-based prior studies to investigate the impact of industry on an organisation's propensity to adopt extensive environmental practices. An industry's pollution profile is, in particular, relevant to the environmental strategies of its participating organisations. Industries involving chemicals, natural resources, and transportation are among those perceived by the public as environmental-damaging (Holmes, 1976; Brammer and Pavelin, 2004; 2006). These industries, due to the nature of their business operations, are also more likely to experience more environmental crises/incidents that, in turn, lead to stronger pressures from relevant stakeholder groups for improvement in environmental performance (Greening and Gray, 1994). Thus, externally-driven societal pressures are exerted on these industries to take environmental issues more seriously (Abouzeid and Weaver, 1978; Shetty, 1979) and to take action to correct, or mitigate, negative public perceptions (Brammer and Pavelin, 2004; 2006). Empirically, most studies have found evidence that industry is a significant determinant of the type of environmental strategy adopted by an organisation, or its orientation/attitude towards environmental issues (Holmes, 1976; Sharma, 2000; Brammer and Pavelin, 2004). In the management literature, it has been suggested that organisations operating in the same sectors/fields adopt similar accounting practices and systems to achieve legitimacy (DiMaggio and Powell, 1983; Meyer, 1986; Richardson, 1987). 


\section{b. Stakeholder pressures}

The management literature also considers other external societal pressures, including those exerted by the media, stakeholders and community, to be strong factors influencing environmental strategy and responsiveness. Henriques and Sadorsky (1996) highlight that environmental plan formulation is positively influenced by customer, shareholder, government regulatory, neighbourhood, and community group pressures, but is negatively influenced by other lobby group pressures. In contrast, Bansal (2005) suggests that corporate sustainability development is positively correlated with media pressure and mimicry with competitors and other organisations in the field and that, over time, there is greater uptake of sustainability practices due to increased community concern for social and environmental issues. Céspedes-Lorente, Burgos-Jiménez, and Álvarez-Gil (2003) also indicate a positive correlation between the power of stakeholders and extensive environmental management practices. However, stakeholder pressures are not found to be a significant driver of environmental innovation in a study by Eiadat et al. (2008) of chemical companies in Jordan.

\section{c. Field and peer pressures}

Peer groups and other companies in the organisational field can provide additional pressure for organisations to adopt environmental practices. Goodstein's (1994) study reveals that organisations are more likely to adopt a child care practice if other organisations in the same field/industry have already adopted this practice. Bansal and Roth (2000) argues that high field inter-connectedness reduces organisations' attempts to stand out from others, resulting in less incentive to adopt product environmental lifecycle analysis. Other studies suggest that membership of a particular field group exerts a significant impact on organisations' environmental management. Álvarez-Gil et al. (2001)'s survey of Spanish hotel industry indicates organisations that are part of a hotel chain, or affiliation, are more likely to adopt formal and extensive environmental management practices. Collins et al (2007) survey finds that New Zealand organisations that participate in the Sustainable Business Network (SBN) are more likely to employ sustainability practices, than those that don't. However, Cole, Elliot and Shimamoto's (2006) study of Japanese firms indicate that Keiretsu membership (a group with strict liquidity requirements) limits a firm's ability to employ extensive environmental management practices.

The link between external societal pressures and organisational MCS is examined in a few accounting studies. Brownell (1985) argues that organisations operating in complex environments use broad-scoped, externally-oriented MCS information to meet the 
multiple demands from different stakeholders in their environments. Additionally, the accounting literature suggests that field and peer pressures are also the significant drivers of organisational MCS. Christensen (2005) argues that the adoption of accrual accounting in the public sector is driven by organisations mimicking successful peers, and that private sector accounting firms substantially influence the promotion and implementation of such accrual accounting. Qian and Burritt (2008) also find that organisations adopt similar environmental management accounting systems as peer organisations operating in the same field. The latter two studies use an IT-based framework to argue that organisations adopt particular MCSs in order to satisfy the dominant pressures and expectations within the organisational field and wider society and thus gain or enhance their legitimacy (DiMaggio and Powell, 1983).

The prior literature above therefore suggests that environmental regulation (Section 3.2.1), external economic volatilities (Section 3.2.2) and industry-related societal pressures, stakeholder pressures, peer and field pressures (Section 3.2.3) represent the externally-driven economic and societal factors that influence organisations' environmental strategies and organisational MCS. In addition to these external factors, internal factors are also important drivers of organisations' environmental strategies. These factors are both human- and non-human-related, as reviewed in the next section.

\subsection{Internal drivers of environmental strategies: Internal}

\section{capabilities and characteristics}

\subsubsection{Organisational size}

Utilising IT, a number of studies have suggested organisational size influences how and when organisations respond to environmental issues. Bansal (1995) argues that large organisations are more visible and as they can be easily seen by relevant constituents, they are easily hurt by these constituents' actions. Therefore, these organisations need to respond to constituents' demands and pursue environmental responsiveness in order to maintain legitimacy (Oliver, 1991; Goodstein, 1994; Ingram and Simons, 1995). From a RBT perspective, Bowen (2000) suggests that high visibility gives large organisations a capacity to influence important constituents, such as the government, regulators, the media, and the community. As an example, electricity firms can use their visibility in the media to alert the public and the government to their opinions in relation to GCCPs and its impacts. In doing so, they influence the public's perceptions and potentially manipulate policy design to their advantages. Additionally, larger organisations have more resource availability and can afford a formal environmental plan, providing them 
with a basis to undertake more extensive and advanced environmental management practices (Bowen, 2000; Álvarez Gil et al., 2001).

Size is also a significant determinant in an organisation's environment-related accounting policy and practices. Brammer and Pavelin (2008) suggest the quality of voluntary environmental disclosure is determined by an organisation's size and the nature of its business activities. Specifically, high quality disclosure is found to be associated with larger organisations and those in sectors most closely related to environmental concerns. Similarly, Cole et al. (2006) examine environmental disclosure, as one of several environmental management practices, and find it to be positively associated with organisational size.

All of the preceding studies indicate that larger organisations are exposed to high societal pressures due to visibility and have more resources. Both of these factors appear to lead organisations to adopt more active environmental strategies and undertake voluntary and extensive environmental disclosure. Additionally, the literature also suggests that technological and financial capabilities affect environmental strategies and associated organisational MCS.

\subsubsection{Technological and financial capabilities}

From a RBT perspective, technical and financial capabilities are critical to enabling an organisation's environmental strategy. James et al. (1999) identify the key barriers to innovative environmental management practices as: costs of the practice; technological availability (particularly important for capital-intensive industries); and financial resource availability (particularly important for service and financial sectors). Consistent with findings by James et al. (1999), Tilley (1999) indicates that organisations resist eco-friendly practices for three reasons, including: i) low ecoliteracy of the owner-manager; ii) the perceived high cost of these practices, and iii) inadequate existing infrastructure. Similarly, Collins et al. (2007) provide evidence that the key barriers to adoption of sustainability practices include high costs and other priorities in resource allocation. Berrone et al. (2007) argue that while IT explains how environmental regulation can force organisations to develop environmental innovation, the extent of innovation depends on availability of organisational resources, capital and R\&D intensity, and organisational slack. Therefore, these studies generally support the idea that innovative and sustainable environmental practices require high financial and/or technological availability. 


\subsubsection{Managerial factors}

Prior management literature contains much evidence regarding the importance of managerial factors to environmental strategy formulation and implementation. Holmes (1976) suggests that management interest in social and environmental issues is one of the key drivers to an organisation's involvement in a corporate responsibility programme. Similarly, Hunt and Auster (1990) and Doonan et al. (2005) argue that managerial support and commitment enable an organisation to pursue an innovative environmental profile and achieve high environmental performance. Henriques and Sadorsky (1996) find that managerial perception of stakeholder pressures positively influences an organisation's formulation of a formal environmental plan. Specifically, organisations whose managers consider these pressures to be high are more likely to have an environmental plan. James et al. (1999) similarly consider leadership as the top enabler/facilitator for the formulation of a formal environmental policy. Sharma (2000) suggests that managerial interpretation of environmental issues (as threats or opportunities) influences the choice of environmental strategy significantly. When managers perceive environmental issues as presenting more opportunities than threats, they are more likely to exercise strategic choice and adopt voluntary action for environmental protection. In contrast, when these issues are primarily considered as threats, a conformance strategy to the societal pressures associated with those policies is likely to be preferred (Sharma, 2000). In de Bakker, Fisscher and Brack (2002)'s study, managerial ability to identify and build required capabilities is found to be a key internal resource to enable a product-oriented environmental strategy. Additionally, Eiadat et al. (2008) argue that managers, who believe that environmental issues should be a top priority and possess environmental knowledge and skills, are the agents to trigger organisational adoption of an innovative environmental strategy. All of these studies, while varying in the choice of specific managerial and human factors to examine, agree that management support, commitment, knowledge, and capabilities are the key enablers of a voluntary and innovative environmental strategy.

\subsubsection{Prior experience, organisational learning, and organisational}

\section{culture}

Prior studies suggest that organisational learning and prior experience in previous environmental projects and a supportive organisational culture are important internal capabilities that enable organisations to pursue an appropriate environmental strategy. For example, Marcus (1988) suggests that organisations with wider prior experience in different environmental activities have more discretion and flexibility in responding to 
external pressures. Therefore, they can exercise autonomous choice in relation to their environmental strategy (Marcus, 1988). Sharma and Vredenberg's (1998) comparative study of seven firms in the Canadian oil and gas industry suggests that continuous innovation and organisational learning through continuous knowledge exchange and formulisation enables the pursuit of innovative environmental strategy that can lead to competitive advantages. Céspedes-Lorente et al. (2003) survey 270 hotels in Spain and find that prior experience with environmental projects provides a foundation for organisations to undertake more extensive practices and initiatives. Claver et al. (2007), investigating a single organisation, find that an environmental strategy has a positive impact on organisational performance when it is supported by organisational learning gained through prior emissions-reduction focused projects. However, the literature has little evidence of the impact of organisational culture on environmental strategy, except for study by Henriques and Sadorsky (1996). Henriques and Sadorsky argue that organisations need a supportive organisational culture when pursuing an environmental strategy that focuses upon mitigating the organisation's impacts on the environment.

The preceding literature review suggests that external economic and societal drivers have different impacts on the environmental strategies chosen by organisations. Additionally, variations in internal characteristics and capabilities can lead to different types of environment-related strategic responses across different organisations. Hence, it is important to understand the types of environmental strategies that an organisation can adopt in managing its environmental performance and responding to these external and internal drivers. The next section reviews the literature to gain an insight into these types of environmental strategies.

\subsection{Types of environmental strategies}

Prior studies in the environmental management literature have proposed a number of ways to categorise environmental strategies. Most focus on identifying an organisation's strategic posture/positioning in relation to environmental issues and environmental context. Dutton and Duncan (1987) suggest that organisations can adopt four different strategies in response to issues that have strategic implications for their organisational performance. These strategies include No response, Resistance, Opportunistic, and Strategic change. Hunt and Auster (1990) propose five different stages of development of an organisation's environmental management system (EMS) that have different levels of impact on the reduction of environmental risks. These five stages are: Beginner (no protection); Fire fighter (minimal protection); Concerned citizen (moderate 
protection); Pragmatist (comprehensive protection); and Proactivist (maximum protection). Steger (1993) presents a matrix view of environmental and market opportunities from which four strategies are derived: indifferent; defensive; offensive; and innovative. From a different perspective, Oliver (1991) suggests several possible strategic responses to environment-related societal pressures. These responses include: acquiescence or compliance; compromise or negotiating institutional demands; avoidance or decoupling parts of the firm; defiance or publicly demonstrating resistance; and manipulation or controlling the environment (Oliver, 1991).

Other studies propose simpler categorisations of environmental strategies. Russo and Fouts (1997) use a dichotomy of environmental strategies: reactive and proactive. Hart (1995) provides three types of proactive environmental strategies organisations can employ to gain a green competitive advantage: pollution prevention, product stewardship and sustainable development. Jansson et al. (2000) divide environmental strategies into three types: reactive, active and proactive. Sharma (2000) uses a simpler categorisation that employs 'compliance' and 'voluntary' to describe organisational strategy in response to the perception of environment-related threats and opportunities. In contrast to other studies which focus on either the production or product development functions, Azzone and Bertelè (1994) consider the involvement of other organisational functions in the environmental strategy process. They suggest five corporate strategic responses matching with five environmental contexts: stable, reactive, anticipatory, proactive and creative.

The categorising schemes proposed by the above studies vary in terms of the external and internal factors they take into account to derive the potential environmental strategic postures and responses. However, most categories deal with managerial interpretation of environmental issues as either threats/constraints, or opportunities. Further, they are significantly similar in the respect that they all rank environmental strategies along a continuum from no response, to reactive and to proactive. A no-response environmental strategy indicates that the organisation is doing nothing to control and reduce the impact of its activities and operations on the natural environment. It is also indicative of not reacting to environment-related pressures and legislation/regulation (Dutton and Ducan, 1987). A reactive environmental strategy focuses on passively reacting to, and meeting the minimal requirements of environmental regulations (e.g. pollution control for compliance purposes). Such a strategy also involves taking action only after the legislation/regulation has been enforced (Hunt and Auster, 1990; Azzone and Bertelè, 1994). Conversely, a proactive environmental strategy aims to monitor and reduce 
environmental impacts beyond regulatory requirements and develop low-impact technologies in-house, or in cooperation with strategic partners (Dutton and Ducan, 1987; Azzone and Bertelè, 1994). A proactive strategy requires organisations to anticipate and act early to respond to threats and/or take up opportunities offered by potential environmental legislation/regulation. Therefore, this would involve lobbying activities to delay or accelerate the introduction of environmental regulation, and product design and marketing initiatives to promote green products and enhance market competitiveness (Hart, 1995; Russo and Fouts, 1997). These types of environmental strategies, in turn, will have different implications for organisational MCS, as discussed in the next section.

\subsection{Organisational MCS}

Environmental management studies suggest that the organisational MCS plays a key role in promoting environmentally-driven business development (Jansson et al., 2000). Environment-focused MCS cannot lead a life of their own and therefore must be integrated with other internal control systems when implementing the environmental strategies chosen by the organisation (Willits and Giuntini, 1994). The literature provides theoretical and empirical evidence for different MCS design, use and objectives to support an organisation's environmental strategies.

\subsubsection{Design of MCS to support environmental strategy}

Prior studies suggest that a number of MCS components are relevant when implementing environmental strategies: informational scope and budgetary controls; an environmental performance management system and environmental accounting; and, communication and coordination systems. Each MCS component and its relationship with environmental strategies are discussed below.

\section{a. Informational scope and budgetary controls}

The link between environmental strategies and organisational MCS has not been systematically examined in the management or accounting literature. However, the relationship between business strategy and MCS is a widely researched topic, as reviewed in Langfield-Smith (1997) and Chenhall (2003). Overall, the MCS literature suggests that organisations which follow a prospector, product differentiation strategy, adopt a more open and broader scoped MCS as well as use less rigid budgetary controls. Conversely, organisations that pursue a cost leadership, defender strategy use a formal MCS that incorporates goals, budgets, and budgetary controls. However, these findings have not been examined in the context of environmental issues and regulations. 
Therefore, little is known about how environmental strategies influence the scope of MCS information and use of budgetary controls.

\section{b. Environmental performance management system and environmental accounting}

Some studies focus on the design of performance management and accounting systems to enable efficient and effective implementation of environmental strategies (Christopher, 1977; Hutchinson, 1996). Christopher (1977) describes the key performance management systems required for organisational success. He argues that such a system should include measures and monitoring systems for changes in the economic, social, political, and ecological environments and the audits of programmes implemented to respond to these environmental changes. Additionally, Hutchinson (1996) suggests that when accounting for environmental costs and savings, it is critical to integrate environmental policy into business strategy. Bebbington and LarrinagaGonzalez (2008) discuss the potential performance impacts of climate change policies such as an ETS. They propose that to effectively manage performance, organisations need to change their accounting practices, and not only account for carbon costs, but also capture and monitor the potential market volatilities and opportunities associated with an ETS. However, there is a lack of empirical evidence regarding the extent that environmental accounting and environment-related MCS components are implemented by organisations in practice. In particular, little is understood regarding the degree to which an environmentally-informed MCS might influence an organisation's ability to manage the impacts of environmental legislation/regulation.

\section{c. Communication and coordination systems}

Prior literature suggests a need for communication and coordination systems across functions and management levels to enable efficient implementation of environmental strategy (Gupta, 1995; Hart, 1995; Judge and Douglas, 1998). Gupta (1995) argues, theoretically, that the integration of environmental strategy into operations management is critical for a proactive approach to environmental management. Such integration requires a clear and direct link between the environmental planning process and senior management. There is also a need for a formal channel through which the corporate environmental group can contribute to the strategic planning process associated with environmental management (Gupta, 1995). Different communication systems enable coordination and cooperation across functions and hierarchical levels, thus, improving cost efficiency in environmental management. Similarly, Hart (1995) argues that crossfunctional cooperation and management in green product design and development is 
critical to the success of a product stewardship environmental strategy. Further, Judge and Douglas (1998) find empirical evidence that coordination between the environmental function and other functions is positively associated with the level of involvement of environmental personnel in the strategic process. This, in turn, drives improved environmental and financial performance. Nonetheless, while discussing the importance of communication and coordination systems, none of these studies empirically examines how the importance of such MCS components varies across organisations that have different environmental strategies and are exposed to differing external environment-related volatilities and pressures such as those related to the GCCPs.

\subsubsection{MCS use to enable environmental strategy}

In order to effectively implement chosen environmental strategies, organisations need to not only establish appropriate controls and control systems, but also use them in an appropriate manner (Langfield-Smith, 1997; Chenhall, 2003; Chapman, 2005). The literature provides evidence of different ways MCS can be used to support chosen environmental strategies. These uses range from the style of MCS use to the use of environmental information for internal decision making and external disclosure, as discussed below.

\section{a. Style of MCS use}

Simons (1991) suggests four types of formal control systems that are associated with strategy planning and control: belief system, boundary controls, diagnostic control systems and interactive control systems. Belief systems are used to define basic values, purposes and mission statements of the organisation and communicate them to lower organisational levels. Boundary controls specify the limits, practices and rules that need to be followed and the risks to be avoided. In contrast, diagnostic control systems are formal feedback systems used by managers to monitor organisational performance against the set targets and to undertake corrective action. Simons argues that any of these three types of control systems can be used interactively by top managers (i.e. interactive control systems) to engage with lower management decision-making processes and thereby encourage learning and dialogue that facilitates strategic renewal. Consistent with Simons (1991), the study by Abernethy and Brownell (1999) finds that hospitals which undergo strategic change and move to a more prospector type of strategy use budgets interactively to encourage dialogue and organisational learning. While being used extensively in the MCS literature, Simons' (1991) framework has not been employed and tested in environmental management studies. 


\section{b. Use of environmental information for internal decision-making}

The use of environmental information for internal decision-making such as strategic planning, budgeting and performance evaluation is also an essential element in implementing environmental strategies. Bungay and Goold (1991) argue that organisations need to integrate non-financial performance measures and targets into strategic planning process in order to sustain a long-term competitive advantage. Similarly, Judge and Douglas (1998) and Sharma (2000) find that such integration is positively associated with organisational financial performance and the amount of resources allocated to environmental issues. Further, organisations need to incorporate environmental measures into budgeting so as to give line-managers adequate financial resources and support (Bungay and Goold, 1991). Environmental measures also need to be used in performance evaluation to motivate the consideration of environmental issues in managerial decision-making (Hutchinson, 1996).

\section{c. Use of environmental information in external disclosure}

The use of environmental information and measures in external reporting is another important element to ensure effective implementation of environmental strategy. Hutchinson (1996) indicates that, in order to integrate environmental policy into business strategy, organisations need to disclose environmental performance in annual reports. Orlitzky, Schmidt, and Rynes (2003) provide evidence that the adoption of CSR leads to improved financial performance when organisations disclose their CSR practices extensively and thus, communicate their social and environmental responsibility effectively to external stakeholders. Aerts and Cormier (2009) suggest organisations manage their environmental performance and public perception of such performance through media press releases and annual reports. Their study finds that perceived organisational legitimacy is positively affected by the extent and quality of the economic-based segments of environmental disclosures in annual reports and by reactive environmental press releases. Overall, these studies indicate that environmental disclosure helps organisations manage their environment-related reputation and contributes to the enhancement of organisational financial performance.

\subsubsection{Objectives of organisational MCS}

The literature suggests that organisational MCS can be used to achieve different objectives. The economic objectives of MCS include ensuring internal efficiency, enhancing competitiveness in the market place, and managing external uncertainty (Bowman and Haire, 1975; Hunt and Auster, 1990; Hart, 1995; Otley, 1999). Additionally, MCS can be used to legitimise and rationalise, or to serve political 
purposes (Brunsson, 1989; DiMaggio and Powell, 1991; Scott, 2001). Prior studies also suggest that these objectives are, simultaneously, the motivations underlying the choice of environmental strategies (Bansal and Roth, 2000; Garriga and Melé, 2004; Barnett, 2007). Each of these objectives, in relation to MCS design and use, is discussed next.

\section{a. Ensuring internal efficiency}

Most of the studies that examine technical uses of organisational MCS are based on contingency theory. Contingency theory suggests there needs to be a match between MCS and technical organisational contexts and work characteristics in order to maximise financial performance (Otley, 1980; 1999). Accordingly, organisational MCS is used to provide information to enable control of existing strategy, operations and activities and planning for future strategy and actions (see the review in Chenhall, 2003). Control is achieved through the provision of past performance indicators which allow the existing strategy and performance to be evaluated and corrective actions to be taken where appropriate. Planning is achieved through control systems providing information to support rational resource allocation with an aim to achieve the organisations' strategy objectives (Anthony, 1965; Burchell et al., 1980). In this respect, the role of an MCS is to ensure that an organisation's strategies are implemented in the most efficient and effective manner. However, a common problem with studies that use contingency theory to explain MCS design and use is a failure to provide a theoretical underpinning to explain exactly through which mechanisms MCS can lead to improvement in organisational efficiency (Spekle, 2001).

\section{b. Enhancing market competitiveness}

An organisation's MCS can be used to enable organisational learning and strategic renewal, thereby maintaining and enhancing market competitiveness (Simons, 1991). By monitoring and collecting information relating to environmental changes, organisational MCS helps managers evaluate and make decisions in relation to future strategy choices so as to gain and maintain market competitiveness (Simons, 1991). Furthermore, it is suggested that an appropriate MCS can promote communication and coordination between environmental functions and business units and increase environmental awareness or commitment throughout the organisation (Gupta, 1995; Hart, 1995). This, in turn, improves environmental performance, organisational reputation and brand image, and enables a green competitive advantage to be gained (Judge and Douglas, 1998). 


\section{c. Managing external uncertainty}

While contingency-based studies assume that the use of organisational MCS depends on the level of external uncertainty faced by an organisation, few have explicitly examined how MCS enables the management and reduction of uncertainty. Hunt and Auster (1990) suggest that by having control systems that monitor and assess vulnerabilities beyond government requirements, organisations can anticipate external uncertainty and formulate appropriate strategies accordingly. Bowman and Haire (1975) argue that CSR reporting can help manage external uncertainties by providing information that is likely to be relevant to all key organisational stakeholders. Taking an IT approach, DiMaggio and Powell (1983) and Oliver (1991), among others, argue that in conditions of high uncertainty, such uncertainty is managed by organisations imitating each other. Such mimicking may result in organisations adopting similar accounting practices and MCS (Abernethy and Chua, 1996; Tsamenyi, Cullen, and González, 2006).

\section{d. Legitimising and rationalising}

Employing an IT perspective, researchers have found that organisational MCS, including accounting practices, have non-economic objectives. Accordingly, MCS could be seen as a 'rational myth' that maintains, and confers social legitimacy on the organisation and its participants' actions. This is achieved by legitimising past actions and providing a frame for rationalising future decisions (Meyer and Rowan, 1991; Baxter and Chua, 2003). Burchell et al. (1980) argue that accounting is influenced by the way it is institutionalised in organisations, such as accounting departments and professional bodies, and by the objectification of accounting knowledge through books and training. Miller (1991) examines the use of discounted cash flow techniques in the UK public sector in the 1960s and finds that they provide the language to rationalise government policies and encourage organisations' investment activities. Similarly, Covaleski, Dirsmith and Michelman (1993) demonstrate how different accounting systems were used as ceremonial systems to create a sense of order and meaning in the heavily institutionalised environments of US hospitals. Furthermore, accounting provides a record of activities in a way that is acceptable and reasonable to external constituents such as the government (Covaleski et al., 1993).

\section{e. Serving political purposes}

The literature also suggests that MCS can be used for political purposes. Burchell et al. (1980) suggest that where there are conflicts between values and interests of participants in the decision-making process, accounting arises as a mechanism through which different parties seek to promote their own concerns, interests and positions. Consistent 
with this argument, Brunsson (1989) argues that budgets have relatively loose links with actions and thus are seen more in terms of talks and decisions. This makes budgets "a good instrument for conducting politics and producing hypocrisy" (pp. 105-106). In support, Hopwood (1990) suggests that as a calculative practice, accounting can be used by organisations to enhance the salience and visibility of particular issues and modify public policies for organisational advantage. Similarly, Ogden (1997) finds that new forms of accounting, such as performance measures, can be used to link political objectives to organisational functioning. In doing so, accounting and associated MCS enable indirect control by the government over businesses.

\subsection{Evaluation of prior literature and identification of remaining} gaps

In summary, the body of literature on environmental management is strong theoretically and empirically regarding the impact of external and internal factors on the choice of environmental strategies. It is apparent from this literature that environmental regulation is a significant trigger of environmental management in addition to other economic volatilities. Prior studies also suggest that external institutional factors, such as stakeholder pressures, field and peer pressures, as well as internal capabilities and characteristics are important in making decisions about environmental strategies. Many types of environmental strategies are found in the literature, ranging from non-response to reactive and proactive approaches. Previous studies indicate the MCS required to support efficient and effective implementation of environmental strategies varies in design, use and objectives. Further, the body of literature on MCS, in general, contends that there are alternative uses of organisational MCS in addition to those that serve economic objectives. Those alternative uses include managing external uncertainty, legitimising past actions and rationalising future decisions, and serving political purposes.

However, there appear to be four major gaps in the reviewed literature.

Gap 1: The literature has not considered environmental strategies in relation to, and in the event of the GCCPs, such as a carbon tax or an ETS. The consequence of this is we do not know whether the environmental strategies and the MCS choices organisations make in response to the GCCPs are similar, or different to those which are used in response to other types of environmental regulation. The potential wide-ranging economic, social, and political impacts of the GCCPs, as 
discussed in Chapter 2, suggest that responses undertaken by organisations will be on a different scale and at a different level than what they traditionally would be.

Gap 2: There is a lack of theoretical underpinnings to explain the mechanisms by which economic factors influence environmental strategy and MCS component choice. In other words, the literature does not provide sound theoretical economic explanations of how environmental regulations with strong economic impacts, such as the GCCPs, drive the choice of environmental strategy and MCS components. Further, examination is also required into how internal economic factors such as technology and infrastructure affect environmental strategy. Additionally, little understanding is available regarding how a particular choice of environmental strategy and MCS components enables the achievement of operational efficiency, and thus financial performance.

Gap 3: The literature is predominantly cross-sectional and has considered different external and internal drivers to environmental management in parallel and in isolation from each other. Correspondingly, we know little about the impact of environmental regulation on organisations' external environments (3a). Furthermore, little understanding exists in terms of the potential inter-relationships between external and internal drivers (3b) and the role of such inter-relationships in driving environmental strategies and organisational MCS (3c). For example, do internal characteristics and capabilities influence an organisation's perceived exposure to external environment-related volatilities and pressures? Additionally, there is also a lack of insight into how a longitudinal period of environmental regulation changes such as the GCCPs, affect organisations' environmental strategies and MCS (3d).

Gap 4: The link between environmental strategies and organisational MCS has not been adequately explored. Previous studies examine the MCS design and use that are required to achieve a given environmental strategy at a particular point in time. However, no insight is available regarding the implications of a change in GCCPrelated environmental strategy for organisational MCS. In other words, do MCS design and use need to change to support the new strategy? Further, prior literature fails to identify what types of controls and control systems support strategy implementation and what types of controls and control systems enable strategy renewal. Thus, little is known about whether organisational MCS needs to fit with a given strategy, or serves to assist future strategy planning. Few insights are also available regarding the motivations and objectives underlying MCS design and use 
under a GCCP context. For example, is the MCS used to fulfil all the objectives identified in the previous studies, or just some of them? Also, what drives one particular objective to be more important than others within the organisation?

A summary of what has been found in the literature and what is yet to be explored in relation to environmental strategies and MCS is provided in Figure 3-1.

Figure 3-1: Summary of findings and gaps from the prior literature

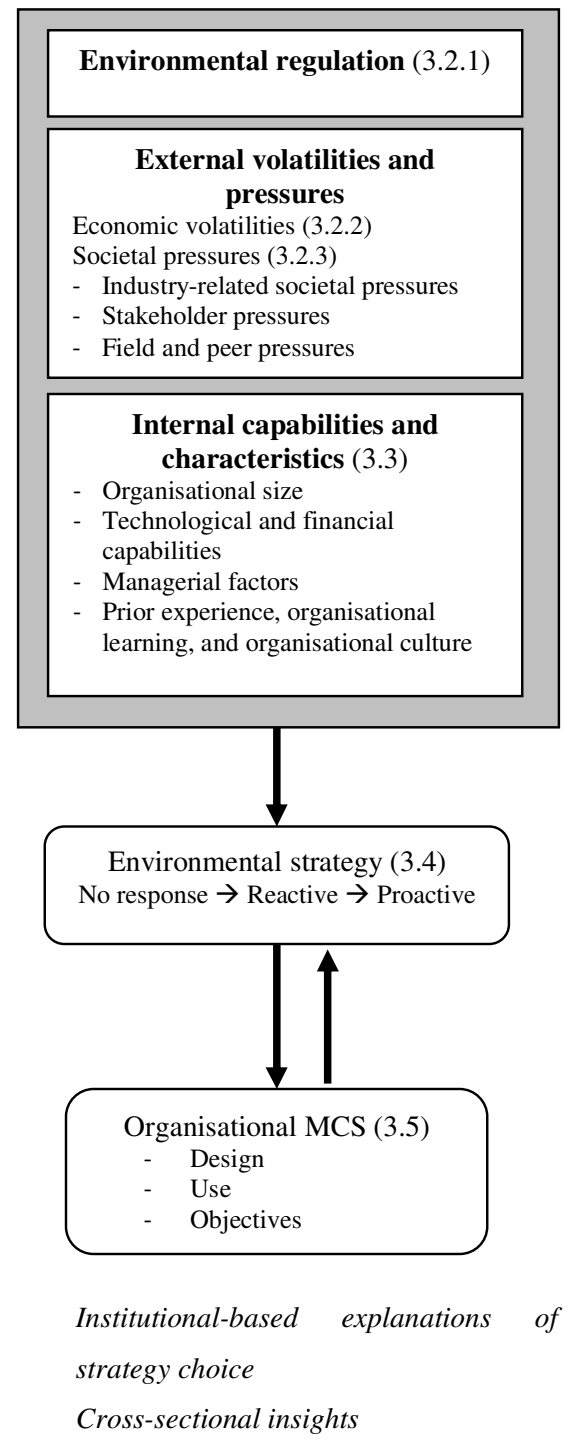

Findings from prior literature

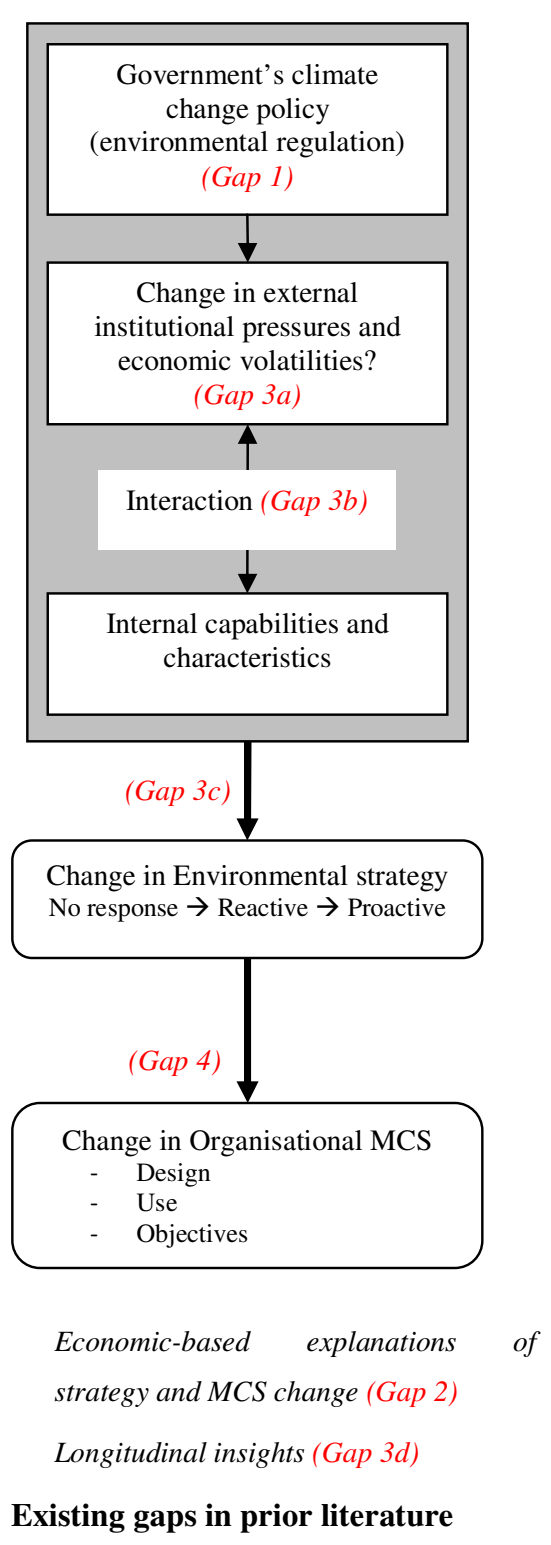

Existing gaps in prior literature

Note: the arrows do not indicate cause and effect relationships

\subsection{Summary}

To summarise, this chapter has reviewed the prior literature and suggested that previous studies have provided limited theoretical and empirical evidence about the implications 
of environmental regulation, and related external and internal drivers for environmental strategies and organisational MCS. This chapter has also identified a number of remaining gaps in the existing literature, thereby providing the theoretical motivations to this study as outlined in Chapter 1 . Specifically, this study contributes to addressing some of the above gaps by choosing the context of the GCCPs to understand the choice and change of environmental strategies under the impacts of environmental regulation (Gap 1). The study also seeks to develop an explanation regarding the influence of environmental regulations with strong economic impacts on strategy and MCS choice, through the application of appropriate economically-based theories in addition to those with an institutional focus (Gap 2). Placing this study in a GCCP context also enables insights to be gained about the potential impacts of the GCCPs on the economic and societal environments in which organisations operate (Gap 3a). Additionally, a basis for improved understanding of the potential interaction between external and internal drivers (Gap 3b) and the impact of such interaction on organisations' environmental strategic and MCS responses (Gap 3c) is provided. Doing this, longitudinal insights into the influence of environmental regulatory change on environmental strategies and MCS can be gained (Gap 3d). There is also a potential to further understand the role of organisational MCS in relation to environmental strategies (Gap 4). This enables the identification of the implications of environmental strategies in response to the changing GCCPs for MCS design, use, and objectives. Furthermore, this study is motivated by a potential to develop a theoretical framework that explains how organisations internally adapt to external environmental regulatory changes.

Based on these theoretical motivations, this study seeks to provide an answer to the following research question:

\section{What are the MCS implications arising from strategy choices New Zealand electricity generators make in managing economic volatility and societal pressures resulting from a prolonged period of GCCP change?}

In order to answer this research question, this study develops a research framework incorporating appropriate theories and the key external and internal drivers, environmental strategy and organisational MCS variables as identified in the literature presented in Chapter 3 above and in Chapter 2. This research framework is developed and presented next in Chapter 4. 


\section{Chapter 4: Research framework}

\subsection{Introduction}

In Chapter 3 the existing research literature was examined in response to the research question identified in Chapter 2. As a result of this examination, four gaps in the environmental strategy and MCS research, as it relates to this study, would appear to exist (refer to Figure 3-1). The purpose of this chapter is to develop a research framework that helps address the research question and narrows the existing gaps in the prior literature. This research framework integrates the external and internal drivers suggested by previous studies as well as the insights from relevant theories to examine the implications for organisational MCS arising from the strategy choices New Zealand generators make in response to the changing GCCPs.

This chapter is organised as follows. The next section outlines the considerations made in choosing the applicable theory/theories and theoretical framework for this study. In developing the theoretical framework, the following section reviews transactional cost economics (TCE), institutional theory (IT) and resource-based theory (RBT) and discusses their inter-relationship and suitability for this study. The research design and framework based on these three theories and prior literature are outlined next. Finally, a summary of the chapter is presented.

\subsection{Considerations in choosing a theoretical framework}

A number of different theories can be employed in organisational research, including contingency theory, agency theory, critical perspectives-based theories, stakeholder theory, TCE, IT and RBT. However, the relationships central to the study and the operating setting within which these relationships exist should determine the choice of theory/theories. The preceding theories are now discussed in those terms in order to identify a framework suitable for this study.

Contingency theory assumes that organisational effectiveness depends on the degree of matching between an organisation's internal characteristics, including strategy and MCS, with contingencies that reflect the economic environments in which the organisation operates (Govindarajan, 1988; Donaldson, 2001; Chenhall, 2003). However, contingency theory does not provide an adequate economics-based explanation as to why organisations have to modify their strategy and MCS choices when external economic environments change. Additionally, where the change is 
externally driven and affects multiple environments, e.g. economic, social and natural environments (which is potentially the case with the ETS), contingency theory does not provide a basis for explaining the interaction between multiple external sources of change as well as assessing the relative pressure that each source may exert on the organisation. Contingency theory views such change from the organisation's perspective, i.e. looking from its internal operating environment out to its external business environment (Donaldson, 2001). This view is organisation-specific and thus will not necessarily capture the variations in transactional and other organisational costs, from one organisation to another, due to each particular source of pressure. Given that these generators differ in generation asset base and ownership structure (Chapter 2), it is expected that GCCP-driven change/pressure will affect each organisation differently. Since this study examines all of the significant generators within the NZEI, it requires a theory that allows a comparative examination of GCCP effects on different organisations and their strategic decision-making. For these reasons, contingency theory is not considered to provide an appropriate framework for this study.

Agency theory explains the existence of MCS as part of an organisation's strategies to monitor and bond the agent's behaviour with shareholders' interests (Watts and Zimmerman, 1986; Yermack, 1995). However, it is not the objective of this study to investigate the changes in organisational MCS to mitigate agency problems. That is, this study is not about assessing the effectiveness of economic interventions, such as the ETS, in providing an incentive for emitters (e.g. generators) to reduce emissions and manage the wider environmental consequences of those emissions. This study examines the impact of regulation change in the form of GCCPs on the strategy choices of generators who are all likely to react differently. Hence, it is not considered to be an agency-constrained study and therefore an agency framework will not be applied here.

Critical perspectives-based theories investigate the use of MCS information for exercising discipline and control over labour (Miller and O'Leary, 1987; Hopper and Amstrong, 1991), or the involvement of micro-politics and internal resistance to the roles of organisational MCS (Wickramasinghe, Hopper, and Rathnasiri, 2004). Labour control and internal politics, albeit important, are not the subject of this study since it investigates the changes in organisational MCS to respond to externally-imposed GCCP-related volatilities and pressures.

Stakeholder theory is interested in identifying who an organisation's key stakeholders are and how its MCS is used to simultaneously address the needs and demands of the different stakeholder groups (Jones, 1995; McWilliams and Siegel, 2001). However, 
this study seeks to examine how organisations modify their MCS to plan for and implement environmental strategies in a GCCP context, rather than to satisfy multiple stakeholder demands and needs. It is noted that stakeholder influences and expectations constitute the institutional environment examined as part of IT. Thus the potential impacts of different stakeholders on organisational MCS are integrated into the ITbased analysis discussed below.

To adequately address this study's research question, the theoretical framework needs to capture the potential and multiple GCCP-driven economic and societal pressures and volatilities in the generators' external and internal environments. The framework also needs to enable a comparative analysis of the differing organisational costs imposed by GCCP-driven changes and pressures on the generators. Further, due to the differences in internal characteristics, the generators are likely to respond to these changes differently, and their strategic and MCS responses may be motivated by different objectives. Nonetheless, a single theory is unlikely to be able to adequately explain the potential multiplicity and complexity in the GCCP-related external environments and their implications for generators' organisational adaptation. Therefore, a multiple-theory framework is required for this study. A multiple-theory framework also allows theory triangulation (Denzin, 2009), which enables an assessment of the relative importance of each change or pressure for an organisation, or the motivations underlying different strategic choices.

Three theories, TCE, IT and RBT, are considered potentially suitable for this multipletheory framework. The implications of changes in the economic environments on organisational costs and internal efficiency are best explained by TCE (Williamson, 1991), while IT provides a basis for understanding GCCP-driven societal changes and pressures and how they affect strategy and MCS choices (Scott, 2001). Further, RBT enables the examination of the impacts of GCCP-driven competitive pressures and internal capabilities on the generators and how strategic choices are made to ensure competitiveness (Barney, 1991). The combined use of these three theories enables coverage of a wider range of GCCP-related economic and societal changes or pressures in the generators' operating environments. It also helps understand how generators react strategically to GCCP-related environments and achieve technical efficiency and market competitiveness while being perceived as legitimate (Hart, 1995; Deephouse, 1999). The next section reviews the three theories and their inter-relationships in order to provide the basis for the development of a multiple-theory framework. 


\subsection{Developing a multiple-theory framework}

The multiple-theory framework developed in this chapter is designed to address the research question, using the different, but complementary, insights from TCE, IT and RBT. These theories are reviewed in the next three sub-sections. Their interrelationships and commonalities are examined next. This is followed by the combination of the three theories to identify the insights they potentially generate in relation to this study's research question.

\subsubsection{Transactional cost economics (TCE)}

Transactional cost economics is an economic theory that argues economising transactional costs is the fundamental driver of organisations' governance structure and associated MCS choice (Williamson, 1975). It explains the optimisation of commercial transactions is based on an assessment of transactions' characteristics and institutional environments. Accordingly, such optimisation is achieved by selecting governance structures and MCSs that match the institutional environments and the characteristics of intra- and inter-organisational transactions.

\subsubsection{Transactional characteristics}

Transactions that are important in understanding governance and MCS decisions are (1) asset specificity, (2) the frequency with which the transactions occur, and (3) the uncertainty or disturbances to which these transactions are subject to. Identifying these different characteristics allows the evaluation of the efficiency of one, or a mixture of modes of governance and MCS types (Williamson, 1996). Furthermore, institutional environments are important considerations in TCE-based strategic and MCS decisions.

\section{Asset specificity}

Asset specificity refers to the degree to which an asset can be redeployed to alternative uses and by alternative users without loss of its productive value (Williamson, 1991). High asset specificity therefore refers to assets whose value will be significantly lost as a result of re-deployment. High specificity assets tend to be purpose built or customised to provide a specific range of benefits to an organisation, or a number of organisations operating in the same industry sector. These types of assets are likely to have higher associated transactional costs due to them requiring a significant investment by the organisation in terms of their design and construction and a reasonably long lifespan. Additionally, the higher the asset specificity, the more likely the values of those assets are exposed to opportunistic expropriation by the parties involved in the transactions, thus causing higher transactional costs. This leads to the need for powerful safeguards 
between the parties. These safeguards may include intensive use of selected interorganisational controls to promote bonding and coordination, and to monitor and prevent opportunism (Spekle, 2001). The use of such controls leads to an increase in transactional costs, but such an increase is more than offset by the avoidance/mitigation of the potential loss in asset value due to opportunism. Therefore, asset specificity is a key determinant in explaining the choice of MCS for economising purposes.

\section{Transactional frequency}

Transactional frequency refers to the intensity with which transactions recur (Williamson, 1985). The more frequently a transaction recurs in relation to an asset of high specificity, the higher the transactional costs incurred due to the increased potential opportunism between the parties involved. In this case, there is an incentive for organisations to adopt elaborate control systems, or internalise the transactions to curb opportunism and ensure smooth coordination in conducting their activities. A high frequency also makes it easier to recover the cost of such elaborate control systems (Williamson, 1985). For example, under an ETS, thermal generators are exposed to higher transactional costs because of the need to regularly measure and monitor generation emissions, and to buy carbon credits to meet ETS compliance obligations. This gives them an incentive to upgrade their emissions monitoring system and establish an in-house carbon trading function. In contrast, when transactions are occasional or idiosyncratic, transactional costs are lower and hence organisations are able to rely on simpler governance and control structures such as market mechanisms and bilateral/trilateral cooperation and trust to govern these transactions (Williamson, 1985). This is potentially the case with some renewable generators who have gained PRE-related carbon credits, but do not have an ETS obligation. To sell these credits, they may use external consultants or brokers or simply utilise current market price systems.

\section{Uncertainty}

Uncertainty can arise from different sources, including market dynamics and complexity, and task unfamiliarity and complexity. A GCCP, such as an ETS, may impose significant uncertainty on thermal generators due to the complexity associated with emissions measurement, monitoring and reporting. They might also experience increased uncertainty due to the lack of expertise if pressured to change from thermal to renewable-based assets. The level of uncertainty is also high for emerging domestic and international carbon markets in which carbon contracts require highly specialised 
knowledge that market participants have not developed. Williamson (1991, 1996) suggests that two possibilities exist when uncertainty increases. The first possibility is that organisations will give up more complex and special design features for more standardised contracts, thereby allowing the continued reliance on market mechanisms, e.g. to monitor their carbon credit buying/selling activities. The second option is to put in place detailed MCS arrangements to enable organisational learning and adjustment to, say, an ETS-focused operating environment. Additionally, organisations can seek compensation from the Government to recover part of the transactional costs they incur as a result of changing their asset and technological base. This may particularly be the case given that the thermally-based generators inherited their thermal assets as part of the commercialisation reforms of the NZEI (Section 2.3).

\section{Institutional environment}

The institutional environment incorporates the "set of fundamental political, social and legal ground rules that establish the basis for production and distribution" (Williamson, 1991, p.287). Williamson (1991) suggests that the institutional environment is a critical factor in economising decisions where the institutional environment dominates governance and controls choices. This suggests that under extreme political, social and economic conditions, efficiency-seeking decisions are not possible and are likely to be replaced with those that are deemed legitimate under the prevailing institutional environments (Roberts and Greenwood, 1997).

Various researchers suggest that it is important to consider organisational MCS design and use within a changing institutional context (Williamson, 1991; Covaleski et al., 2003). Market structures, expectations, rules of competition and forms of transactions become legitimate and require particular types of governance and control choices (Commons, 1934; Coase, 1937). Consequently, when institutional environments change, the definition of economic legitimacy shifts and necessitates appropriate adaptation in organisational MCS (Covaleski et al., 2003). Further, institutional change can lead to changes in transactional characteristics which in turn alters the comparative costs between alternative MCS structures (Williamson, 1991). Hence, under changes in institutional environments, the economic viability of the existing MCS is contested, leading to decisions to replace or modify such an MCS with a new one (Covaleski et al., 2003). 


\subsubsection{TCE in environmental strategy and MCS literature}

As reviewed in Chapter 3 (3.2.1), no study explicitly uses TCE to explain the choice of environmental strategies and organisational MCS in the context of environmental regulation. However, several scholars have argued that TCE is a valuable theoretical tool in examining organisational strategies and MCS (Spicer and Ballew, 1983; Williamson, 1999; Spekle, 2001; Covaleski et al., 2003). TCE argues that economising transactional costs is crucial to achieving economic efficiency, a key condition for organisational survival (Spicer and Ballew, 1983; Spekle, 2001). Thus, a primary TCE theoretical question centres on whether transactions are controlled efficiently through the choice of appropriate strategies and MCS. Consequently, TCE provides a simple framework within which strengths and weaknesses of different strategies and MCS structures can be related and compared (Zimmerman, 1997; Walker, 1998). Additionally, TCE helps identify the key transactional factors that are likely to influence organisational strategy and MCS choices (Spicer and Ballew, 1983; Spekle, 2001). The adoption of TCE also mitigates the current focus of MCS literature on transactions within the organisations and allows a wider coverage of strategy and MCS decisions in both intra and inter-organisational contexts (Covaleski et al., 2003). Therefore, an understanding of how MCS is used to reduce opportunism and optimise efficiency in the transactions involving external organisations, (e.g. carbon credit purchases or partnerships in generation development projects), can be gained.

\subsubsection{The use of TCE to understand GCCP-related strategic and MCS change}

As discussed in Chapter 2, the GCCPs are likely to change the generators' external economic environments, including changing market competition and fuel economics, and expose them to carbon market fluctuations and policy and regulatory change. Additionally, the GCCPs increase the production costs of thermal generators by imposing a carbon charge on their generation activities. Thermal generators will also incur substantial compliance costs associated with the required monitoring, reporting and verification of emissions and carbon credits. Hence, to sustain their existing levels of profitability, thermal generators have to find ways to minimise the production and transactional costs and mitigate the impacts of GCCPs on financial performance. They can do so by formulating appropriate strategies and/or modifying their strategic emphasis. Strategic emphasis refers to the level of importance placed on a particular environmental strategy and hence the amount of resources and personnel channelled into such a strategy to achieve the desired performance outcomes (Ittner and Larcker, 1997). New environmental strategies and/or a strategic emphasis change will lead to 
adaptations in organisational MCS, including the establishment of new control systems, or a change in the use of selected controls.

Strategic and MCS change, in this sense, is the result of rational economic calculations to optimise production and transactional costs and internally adapt to changes in an organisation's economically-focused institutional environments (Williamson, 1991). Furthermore, since this study investigates organisational MCS in a changing institutional environment (i.e. the planned introduction of a GCCP), TCE enables the examination of how existing MCSs remain viable or are changed for internal efficiency purposes (Covaleski et al., 2003). Transactional cost economics also provides a possible explanation to the impacts of GCCP-related transactional characteristics on MCS and strategic change. Consequently, TCE-based economic insights can be gained into the external and internal economic drivers of organisational strategies and associated MCS due to GCCP introduction. However, TCE is not without its disadvantages.

\subsubsection{Disadvantages of TCE}

Despite TCE's focus on efficiency-seeking and transactional optimisation, some researchers argue that cost control and internal efficiency are not always the prevailing concerns in strategy and MCS choices. Instead, organisations may be motivated to adopt controls to conform to institutional expectations (Scott, 2001), or to gain an advantage over their competitors (Porter, 1980; Barney, 1991). Although emphasising transactional characteristics and an economically-focused institutional environment, TCE researchers are criticised for failing to recognise the role of societal and associated institutional pressures on the design and use of organisational MCS (Preston, 1991). The need to respond to institutional pressures is particularly important in the context of the NZEI where the limited number of generators renders them highly sensitive to public scrutiny. Further, the GCCP changes are likely to lead to additional political, social and natural environmental pressures that supplement economic considerations in influencing managerial decisions to change environmental strategy and MCS. Furthermore, competitive electricity markets (Section 2.3.2) require generators to adopt strategies and MCS to maintain competitiveness, in addition to those pressures driven by efficiency-seeking criteria. Furthermore, TCE does not consider how internal resources may be reallocated to enable more effective change management, or how the availability of existing resources may assist some organisations to manage and control their operations better than others.

Institutional theory and RBT allow theoretical explanations that can mitigate the limitations of TCE. Institutional theory potentially provides a means to capture the 
potential impacts of political and social pressures on organisational strategy and its MCS. Resource-based theory is useful because it explains organisational ability to make use of their existing and potential internal resources to optimise internal efficiency, as well as enhance market competitiveness. The next two sections review IT and RBT, and discuss how these two theories add insights that help understand the changes in environmental strategy and MCS of electricity generators under the GCCP.

\subsubsection{Institutional theory (IT)}

There are a number of variants in IT, namely, new institutional (transactional) economics, old institutional economics (Selznick, 1957; Clark, 1960, 1972), and new institutional sociology (Carruthers, 1995). New institutional sociology emphasises legitimacy, the embeddedness of organisational fields, and formal rules, routines and scripts (DiMaggio and Powell, 1991; Greenwood and Hinings, 1996). These rules, routines, and scripts are considered institutions, or "social structures that have attained a high degree of resilience" (Scott, 2001, p.48). This differs from old institutional economics which focuses on issues of power, interests, coalitions and competing values and informal structures (Stinchcombe, 1997; Moll, Burns and Major, 2004). Recently scholars have converged insights from new institutional sociology with those of old institutional economics into neo-institutionalism (hereafter IT) to understand the role played by external institutions as well as intra-organisational routines and institutions in affecting organisational MCS choice and change (Greenwood and Hinings, 1996; Scott, 2001; Fowler, 2009).

Institutional theory argues that organisations exist in a wider environment characterised by a system of societal and institutional values and expectations that shape social reality; commonly referred to as institutional pressures (Meyer and Rowan, 1977; Meyer et al., 1983). Generally, three types of institutional pressures are found at various times and in various combinations. Regulatory or coercive pressures originate from rules and regulations with associated enforcement mechanisms. Normative pressures come from the shared values or norms of a particular group that are internalised by group members and then imposed on others. Cultural-cognitive or mimetic pressures result from the taken-for-granted assumptions made by those within the institutional environment and can result in the mimicking of behaviour and practices that are considered legitimate (Abernethy and Chua, 1996; Scott, 2001; Fowler, 2009). In order to survive, organisations conform to these pressures by shaping their internal structures and systems accordingly, even though such conformity has little to do with organisational efficiency (DiMaggio and Powell, 1983, 1991). 
Institutional theory is often conceptualised as a theory of stability rather than change (Brint and Karabel, 1991; DiMaggio, 1988; Hirsch, 1997). This conceptualisation results from a dominant research focus on the isomorphism commonly found between organisations exposed to strong institutional environments (Orru, Biggart, and Hamilton, 1991). However, other scholars argue that IT can be used to explain organisational change (Dougherty, 1994; Greenwood and Hinings, 1996; Burns and Scapens, 2000). An organisation is assumed to be embedded within its environment and its behaviour is sanctioned and made routine by the norms widely accepted and diffused in such an environment. This embeddedness leads to stability and inertia in organisational structures and arrangements (Tolbert and Zucker, 1983). However, the more environmentally embedded an organisation is, the more uncertainty and instability it will experience when confronting external change, which in turn leads to the need to adapt internally (DiMaggio and Powell, 1991; Greenwood and Hinings, 1996). Additionally, due to the need to maintain legitimacy with external stakeholders, organisations need to react when the wider institutional environment in which organisations are embedded, change (DiMaggio and Powell, 1983, 1991). Such a reaction is primarily realised in the form of adopting new systems and structures that reflect new accepted norms and values (Levi, 1990; Scott, 2001).

\subsubsection{Institutional theory in environmental strategy and MCS literature}

As reviewed in Chapter 3 (3.2), many prior studies use IT to explain the impacts of environmental regulation and societal pressures on environmental strategies and organisational MCS. Prior studies suggest that environmental regulation (Garrod and Chadwick, 1996; Clemens, 1997), industry-related societal pressures (Brammer and Pavelin, 2004; 2006), stakeholder groups (Henriques and Sadorsky, 1996; Bansal, 2005), and peer and field pressures (Bansal and Roth, 2000; Álvarez-Gil et al., 2001) are important drivers of environmental strategies. Further, wider societal expectations, field and peer pressures are found to induce similar accounting practices among different organisations (Meyer, 1986; Ribeiro and Scapens, 2006).

Accordingly, it is argued that organisations adopt the environmental strategies and related MCS that are consistent with institutional and wider societal expectations in order to gain the resources critical for organisational survival. By doing so, they demonstrate conformity to the institutionalised rules and expectations expressed by external constituents and hence gain social legitimacy (Covaleski and Dirsmith, 1983, 1988; Covaleski et al., 1993). In this respect, the adoption of environmental strategies and particular MCSs serve legitimacy purposes rather than organisational efficiency 
purposes (Meyer, 1986; Greening and Gray, 1994). The legitimacy gained from adoption of these strategies and MCS leads to organisational survival, independent of economic performance or internal attributes (Scott, 2001).

In some cases, IT suggests that an organisation's MCS serves a symbolic role. That is, the MCS exists only to signal to external parties and stakeholders organisational legitimacy, and thus adds little to organisational performance improvement (Carruthers, 1995). As the MCS has been adopted to meet institutional expectations without significantly altering the technical processes at the core of the organisation (Meyer and Rowan, 1977), the MCS will become decoupled with actual work activities (Scott, 2001). However, in other cases, MCS changes can be undertaken to gain legitimacy with external stakeholders and to enhance internal efficiency, thereby improving organisational performance (Fernández-Alles and Valle-Cabrera, 2006). Conforming to institutional pressures and expectations and achieving internal efficiency simultaneously are critical requirements for organisations operating in an external environment that is characterised by both institutional and technical elements (Scott, 2001). This is the case with the GCCP-focused environment which exerts strong institutional and economic pressures on generators (Chapter 2).

\subsubsection{The use of IT to understand GCCP-related strategic and MCS change}

As discussed in Chapter 2 (2.3.3), the introduction of the GCCPs is likely to result in significant changes in the generators' institutional environments. Institutional theory predicts that these changes are likely to pose challenges for generators' legitimacy. To remain legitimate, generators should respond to these changes and make choices regarding their environmental strategies and organisational MCS so as to satisfy the GCCP-related societal expectations and demands. Using IT, five possible strategic and MCS responses the generators can use to achieve legitimacy are: acquiescence or compliance; avoidance; compromise or negotiation; defiance; and manipulation or controlling the environment (Oliver, 1991; Scott 2001).

Institutional theory predicts that a conformance strategy is preferred when organisations perceive that the adoption of a particular environmental strategy and associated MCS results in both legitimacy gains and internal cost reductions (Oliver, 1991; Goodstein, 1994). In other cases, organisations may perceive the adoption of particular practices has significant costs, but results in few economic benefits to the organisation (Meyer et al., 1983; Goodstein, 1994). Therefore, organisations may undertake an avoidance strategy, for example, adopting environmental strategies and related MCS ceremonially and within a limited scope. A compromise or negotiation strategy allows a balance to be 
achieved between the elements required by the stakeholders and society and those adopted by organisations (Oliver, 1991). Accounting numbers could be used to support a compromise strategy (Burchell et al., 1980). Another possible strategy is that of defiance where the organisation publicly resists society-imposed institutional pressures (Oliver, 1991). The last potential strategy is one of manipulation where the organisations attain a position of power and are able to manipulate societal expectations and therefore shape what is considered legitimate (Oliver, 1991). Consistent with this, organisations can use accounting to influence and modify public policies and promote their political interests (Hopwood, 1990). However, these different strategic responses have not been subjected to significant empirical testing in institutional research (Poole and Van de Ven, 2004).

\subsubsection{Disadvantages of IT}

Institutional theory is subjected to a number of criticisms. One of the shortcomings of IT is its limited analysis of the process of institutionalisation (Phillips, Lawrence and Hardy, 2004). That is, the process during which routines, scripts and structures become institutions (Scott, 2001). Additionally, most studies have been cross-sectional, measuring isomorphism as outcomes rather than processes (Mizruchi and Fein, 1999). Further, IT has limited capacity to explain incremental change and the co-existence of multiple organisational forms within a field (Kraatz and Zajac, 1996; Fligstein, 1997). Institutional theory-based studies are also criticised as emphasising the channels through which practices are diffused, while underplaying variations in the levels of institutionalisation (Tolbert and Zucker, 1996).

Additionally, while being predominantly focused on social legitimacy, IT-based studies underplay the importance of internal efficiency and external market competitiveness which drive financial performance. Similar to social legitimacy and internal efficiency, market competitiveness is one of the most critical requirements for organisational survival (Hart, 1995; Deephouse, 1996; Kostova, Roth and Dacin, 2008). Resourcebased theory (RBT) explains how organisations can achieve such market competitiveness. It suggests competitiveness is gained through strategic differentiation, which is acquired by possessing and developing strategic resources which are not easily copied or obtainable by other competing organisations (Porter, 1980; Barney, 1991; Lieberman and Asaba, 2006). The next section reviews RBT.

\subsubsection{Resource-based theory (RBT)}

Transactional cost economics explains how organisations choose their environmental strategies and resultant MCS to optimise internal efficiency and cost control, while IT 
emphasises the role of MCS in legitimising organisational operations and activities to the social and political environments in which they operate. However, neither theory considers the need for organisations to stay competitive in their markets as a critical requirement for survival. RBT fills this gap and explains how organisations can maintain and enhance their competitiveness through the appropriate utilisation of their internal resources.

RBT emerges from the concern that a focus on either TCE-based internal efficiency or IT-based social legitimacy is not sufficient to secure sustainable organisational competitiveness. Instead, organisations need to develop resources that are hard to copy or are not easily obtainable by other organisations and adopt isolating mechanisms to create barriers to copying by others (Barney, 1991; 2001). The conditions under which an organisation's resources become competitive capabilities are twofold:

- The first condition is when an organisation nurtures internal competencies and applies them in an appropriate external environment (Barney, 1991). For example, generators can develop staff expertise in renewable energy before an ETS is introduced, and then implement a large-scale renewable investment programme. By doing so, generators build an internal capability that enables them to take advantage of the favourable economics of renewables over fossil fuels.

- The second condition is where existing resources allow an organisation to "exploit opportunities and neutralize threats" in the external environments (Barney, 1991, p.106). For example, a generator with $100 \%$ renewable-based generation assets can take advantage of the opportunities available in a carbon-constrained economy due to its clean and green image.

Therefore, RBT emphasises the fit between what the organisation has the ability to do (internal resources) and what it has the opportunity to do (external environment conditions) (Russo and Fouts, 1997). As discussed in Chapter 2, the GCCPs are likely to create new environmental conditions for generators, such as changing retail competition and changing fuel economics. Based on RBT, it can be argued that generators' competitiveness will depend on whether their existing resources match the new conditions, or whether they can develop appropriate capabilities to respond to changing market conditions.

\subsubsection{RBT in environmental strategy and MCS literature}

As reviewed in Chapter 3 (3.3), many previous studies have used RBT to examine the importance of organisational internal resources and capabilities for environmental 
strategies. These studies indicate that size (Bowen, 1999, 2000; Álvarez Gil et al., 2001) and technological capabilities (James et al., 1999; Collins et al., 2007) are the key nonhuman factors that enable extensive and innovative environmental strategies. Further, human factors are important drivers of the choice and implementation of appropriate environmental strategies. These factors include: prior experience in emissions reduction and other environmental projects (Goodstein, 1994; Marcus, 1988; Céspedes-Lorente et al., 2003), organisational learning (Sharma and Vredenberg, 1998), managerial factors (Hunt and Auster, 1990; Sharma, 2000; de Bakker et al., 2002) and corporate culture (Henriques and Sadorsky, 1996). However, no prior studies appear to use RBT to explain the design and use of organisational MCS, in relation to environmental strategies.

\subsubsection{The use of RBT to understand GCCP-related strategic change}

Resource-based theory is relevant in this study because generators operate in an environment characterised not only by strong institutional pressures, but also by intense competition. In order to ensure their long term survival, organisations need to have a competitive advantage over others in the marketplace (Porter, 1980). Such a competitive advantage is derived from a differentiation strategy that is developed from internal resources and capabilities (Barney, 1991; 2001). Hrebiniak and Joyce (1985) suggest that in regulated and changing industries (such as the NZEI), organisations adopt a differentiated strategic choice. Thus, organisational strategies are chosen based on available organisational resources, but they still conform to external institutional and economic expectations and pressures. Environmental strategies and MCS are considered key mechanisms through which organisations create a competitive advantage while maintaining their external (social) legitimacy and reputation (Westley and Vrendenburg, 1991; Hart, 1995). Hence, organisations, such as the generators, can maximise their revenue flows through competitive differentiation while still being perceived as environmentally responsible by their key stakeholders and the wider community (Deephouse, 1999).

The use of RBT allows the examination of the role played by organisational resources in the choice of environmental strategies and MCS in response to the planned GCCPs. In other words, RBT provides a theoretical base to explain why different generators, with varying resource capacity, may employ different environmental strategies which, in turn, have different implications for organisational MCS. 


\subsubsection{Disadvantages of RBT}

Like the other theories, RBT has its disadvantages. It argues that differences in performance are caused by variations in resources and capabilities across organisations. Thus, RBT is criticised for ignoring industry factors that can have important impacts on organisational performance (Kraaijenbrink, Spender and Groen, 2010). Priem and Butler (2001) argue that sustainable competitive advantage can be turned into a weakness when external environmental conditions change. Others suggest that changing external environments require organisations to develop dynamic capabilities in order to gain a competitive advantage (e.g. Fiol, 2001; Helfat et al., 2007). Connor (2002) contends that RBT applies only to large organisations with significant market power that can rely on stable resources to maintain competitive advantage. In contrast, smaller organisations have to use dynamic resources. Resource-based theory is also criticised for not making a clear distinction between the resources that are inputs to organisations and the capabilities that enable organisations to deploy and utilise those inputs. Additionally, RBT does not address how different types of resources (for example, tangible versus intangible resources, or physical versus human capital) contribute differently to an organisation's competitive advantage (Barney, 1991; Barney and Clark, 2007). Kraaijenbrink et al (2010) suggest that these critiques of RBT can be addressed by the development of RBT to incorporate the concept of dynamic resources and to distinguish between different types of resources and their impacts on organisations' competitive advantage.

\subsubsection{The relationships between TCE, IT and RBT}

In the preceding sections, an overview of TCE, IT and RBT and how their use enables an understanding of environmental strategies and MCS change inside generators in response to GCCPs, has been provided. This section discusses the relationships between the three theories.

\subsubsection{Relationship between TCE and IT}

Institutional theory and TCE are inter-related in a number of ways. Firstly, both theories are interested in institutions and institutional change (Burns and Scapens, 2000). While institutional environments are the key consideration in IT, the importance of socialpolitical dimensions in the institutional environments is implicitly recognised by TCE (Covaleski et al., 2003). Furthermore, many researchers argue that the examination of control choices in an institutional-change context requires the use of TCE and IT perspectives (Williamson, 1991; Oliver, 1991; Goodrick and Salancik, 1996; Covaleski et al., 2003). Institutional change leads to new economic uncertainties and shifts in the 
comparative transactional costs between alternative control choices, thus requiring modifications in organisational MCS in order to maintain efficiency (Williamson, 1991; Goodrick and Salancik, 1996). Institutional change can also generate new social pressures and expectations which demand organisations to adopt appropriate controls and control systems to maintain legitimacy, thus requiring an IT-based explanation (Greenwood and Hinings, 1996). Thus, by employing TCE and IT simultaneously, there is a potential to differentiate the economic versus social significance of MCS change in a GCCP context. Furthermore, it allows the relationship between the GCCPs' economic impacts and societal demands and pressures to be assessed. For example, an assessment can be made as to what extent the desire to achieve a cleaner and lower-emission atmosphere has led society to accept potential electricity price increases due to an ETS.

Secondly, researchers suggest that efficiency and legitimacy are not mutually exclusive, that is, they are two intertwined organisational objectives underlying strategy choice (Fernández-Alles and Valle-Cabrera, 2006) and MCS design and use (Covaleski et al., 2003). In pursuing efficiency, organisations may adopt strategies and practices which, over time, can be diffused to other organisations and businesses and thus gradually become institutionalised and gain legitimacy. Conversely, in seeking legitimacy, organisations undertake strategies that improve trust and relationships with key organisations, thereby facilitating the conduct of their business operations and improving organisational efficiency (Fernández-Alles and Valle-Cabrera, 2006).

Thirdly, while governing/controlling transactions is an economically driven activity, the choice of strategies and MCSs is inevitably constrained by organisations' existing routines, habits and culture as well as the pressures imposed by external institutional environments (Burns and Scapens, 2000; Covaleski et al., 2003). This view is consistent with TCE which suggests that decision makers will choose MCS to achieve the best possible organisational efficiency within institutional, organisational and human constraints, a choice later referred to as constrained efficiency by Roberts and Greenwood (1997). Roberts and Greenwood (1997) argue that organisations are biased towards types of governance and control that are considered legitimate in the institutional environments, but are not rational from an economic perspective.

Consequently, the dual use of TCE and IT in this study is possible because both theories recognise the importance of institutional environments and they provide insights into the relationships between GCCPs' economic impacts and societal pressures/demands within an institutional change context. Furthermore, this use allows the examination of the interaction between legitimacy and efficiency needs and how environmental 
strategies and MCS are established and used to balance these needs. Additionally, potential insights can be gained regarding the impacts of institutional factors and environments on cost-focused economising strategic and MCS decisions.

\subsubsection{Relationship between RBT and IT}

The interface between RBT and IT has been well recognised in the literature. Many researchers suggest that in formulating strategies, corporate management recognises that competitive advantage must be created within a broader scope of social legitimacy (DiMaggio and Powell, 1983; Hart, 1995). This dualism in strategy formulation enables organisations to execute a strategy within the legitimate bounds of their institutional environments. Consequently, the environmental strategies undertaken by organisations are likely to conform to the expectations and pressures of external stakeholders, while being sufficiently differentiated to maintain/gain market competitiveness. This approach is referred to as legitimate differentiation (Deephouse, 1996; 1999). Institutional change potentially modifies the comparative competitiveness between industries and organisations and reduces the strategic discretion available to organisational management (Oliver, 1991; Goodrick and Salancik, 1996). Furthermore, it can be argued that existing resources and capabilities influence the extent to which an organisation achieves social legitimacy (Barney, 1991; 2001). For example, it is easier for a renewable generator to comply with GCCP-focused public pressures for emissions reductions and thus gain social legitimacy than for a thermal-based generator which has substantial generation-related carbon emissions.

\subsubsection{Relationship between RBT and TCE}

The link between RBT and TCE is less well recognised in the literature. Williamson (1999) suggests that TCE can be integrated into strategic management by investigating how organisations, with pre-existing strengths and competencies, organise particular transactions and achieve internal efficiency. This suggestion recognises the role of resource-based concepts in explaining cost-focused strategic decisions and choices of organisational MCS. For example, large generators, with stronger financial and human resource capabilities, are more likely to establish an internal carbon trading team to manage carbon costs. The activities of this team could then be monitored and organised using an extensive MCS containing performance measurement, internal reporting and training systems. Furthermore, existing resources and capabilities can help organisations to achieve organisational efficiency (Barney, 2001). For example, expertise in previous emissions-reduction projects could help generators reduce their emission level and thus lower their carbon-related compliance costs. Similarly, prior investments in wind 
development may help generators gain access to favourable wind sites or low-cost suppliers of wind turbines. Both options can reduce production costs. Therefore, it can be argued that RBT potentially explains why some organisations have lower transactional costs, or can control their production and transactional costs more effectively than others, although they are exposed to a similar external economic environment.

In a GCCP context, TCE supplements RBT by providing an economic dimension to strategic choices that result in a change in internal capabilities and resources. For instance, a thermal generator that decides to develop renewable assets as a result of the GCCPs is likely to experience a shift in transactional costs. While both renewable and thermal assets may have high asset specificity, the move to renewable assets represents reduced GCCP-related compliance costs and relatively lower short-term costs of renewable generation. However, the thermal generator may simultaneously incur increased adaptation and learning costs due to its lack of prior experience in renewable energy. Thus, a RBT-based change in internal capabilities and resources will have economic implications which can be explained from a TCE perspective.

\subsubsection{Commonalities between TCE, IT and RBT}

The above discussion highlights that despite having different assumptions about the objectives underlying the choice of organisational strategies and/or MCS, the three theories, TCE, IT and RBT, share some key commonalities. Firstly, the three theories are concerned with different aspects of organisational performance, whether it is internal efficiency (related to transactional and production costs), competitiveness or social legitimacy. Managing these different aspects of organisational performance is considered critical for organisational survival (DiMaggio and Powell, 1983; Williamson, 1991; Barney, 1991).

Secondly, all the three theories consider the importance of institutional environments in strategic and MCS decisions. Transactional cost economics suggests that strategy and MCS choices need to fit the prevailing social, political and legal environments that provide the basis for production and distribution by organisations (Williamson, 1991; Covaleski et al., 2003). Transactional cost economics also recognises that strategy and MCS decisions for organising purposes are likely to be constrained by institutional factors, such as, existing routines, practices and norms internal to the organisation (Spekle, 2001). Similarly, RBT-based researchers increasingly suggest that competitiveness needs to be created within the legitimate norms of institutional environments (Hart, 1995; Deephouse, 1996). Further, RBT recognises that change in 
institutional environments can change the level of relative competitiveness among organisations, leading to a need for strategy and MCS adaptations (Oliver, 1991; Goodrick and Salancik, 1996).

Thirdly, the importance of internal resources is recognised by all the three theories, though in different ways. Resource-based theory contributes resource-based explanations to how organisations can optimise internal efficiency (from a TCE perspective) and achieve social legitimacy (from an IT view). Transactional cost economics considers that by having different pre-existing strengths and competencies (internal resources), organisations govern and control transactions differently (Williamson, 1999). Additionally, as argued in the previous section, differing resource bases can lead to variations in the level of internal efficiency achieved by different organisations (Williamson, 1999). In a similar manner, some organisations are better at maintaining their social legitimacy than others due to existing internal resources (Barney, 2001). Further, IT contends that organisations need to conform to institutional norms and expectations so that they can maintain/gain access to the resources critical for organisational survival (Meyer et al., 1983; Scott, 2001).

The above discussion highlights that the three theories overlap in three areas: i) organisational performance, ii) institutional environments, and iii) internal resources. However, there are differences between them. In explaining strategy and MCS choices, TCE emphasises transactional cost optimisation and thus internal efficiency as the underlying objective (Section 4.3.1), IT focuses on social legitimacy (Section 4.3.2) while market competitiveness is the key concern in RBT (Section 4.3.3). Further, the three theories differ in the range of factors considered when investigating the drivers of strategy and MCS choice. While the focus of TCE is on transactional characteristics and economic environments, IT captures social and political factors in organisations' external and internal environments and RBT considers that resources and resource availability are the key explanatory factors. Figure 4-1 summarises the overlapping areas of the three theories and highlights their commonalities and differences. 


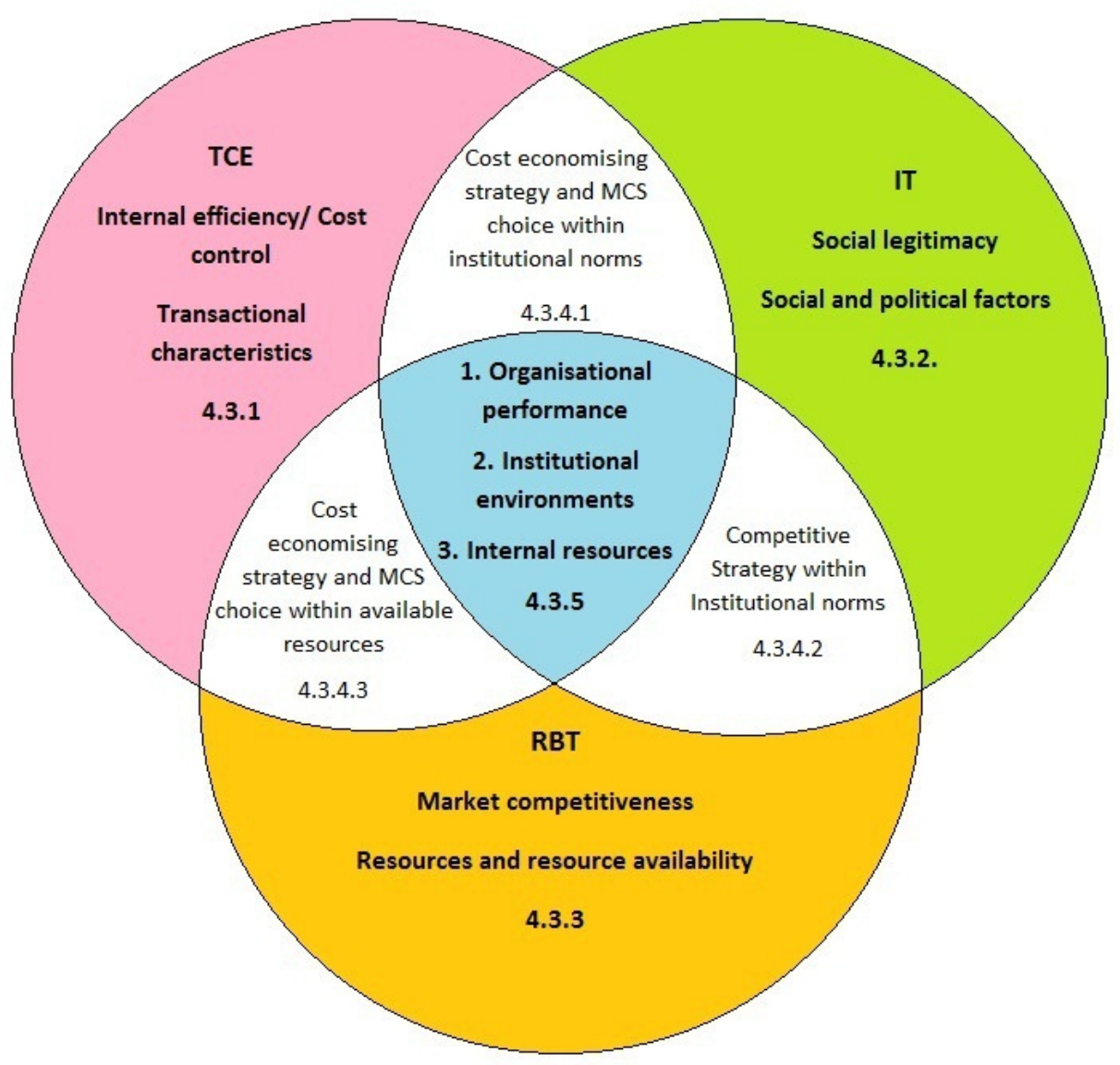

Consequently, it can be argued organisations' strategy and MCS decision-making need to take into consideration the availability of existing internal resources, transactional characteristics and the prevailing conditions and expectations in the economic and institutional environments. Doing this enables organisations to achieve internal efficiency, competitiveness and/or social legitimacy and thus effectively manage organisational performance and ensure survival.

Due to their commonalities and differences, this study adopts TCE, RBT and IT to examine and explain the changes in environmental strategies and organisational MCS to manage the economic and institutional impacts of the GCCPs. The next section discusses this in detail. 


\subsubsection{Integrating TCE, IT and RBT to examine generators' GCCP-related}

environmental strategies and organisational MCS

There are several benefits obtained from integrating TCE, IT and RBT to examine the changes in environmental strategies and organisational MCS generators make in response to the GCCPs. Firstly, like any other business organisation, generators exist within economic and institutional environments. The economic environment exerts technical or competitive pressures, while the institutional environment comprises the wider societal norms, values and requirements which organisations have to conform to in order to be seen as legitimate (Meyer and Rowan, 1977; Granlund and Lukka, 1998; Scapens, 2006). The combined use of TCE, RBT and IT ensures that majority of the different economic, political and social elements in the internal and external operating environments of generators are taken into consideration when answering the research question. Chapter 2 highlights that GCCPs are likely to change the generators' external environments, which will have consequences for their market competitiveness and social reputation. Additionally, the generators' emission profile may lead to high carbon and compliance costs which threaten their internal efficiency. These factors are both economic and societal in nature, and therefore the combined use of TCE, IT and RBT will provide a more comprehensive understanding of the GCCP-related changes in the generators' environments.

Secondly, from an environmental strategy and MCS viewpoint, the integration of these theories allows an examination of the implications of GCCP-related impacts for generators' environmental strategy and MCS choice. Transactional cost economics provides an economic-based explanation of how generators may change their environmental strategies and choose appropriate MCSs in order to minimise transactional costs and optimise internal efficiency. Institutional theory suggests that some generators may choose to undertake particular strategies and MCS decisions in order to secure or enhance their social legitimacy. Resource-based theory indicates that a change in environmental strategies and MCS may be motivated by a need to maintain market competitiveness in a carbon-constrained environment. Resource-based theory also allows an examination of how organisational resources and capabilities influence the ability of a generator to attain internal efficiency (TCE), social legitimacy (IT) and market competitiveness (RBT) in a GCCP context.

Thirdly, by integrating the theoretical insights provided by TCE, IT and RBT, comprehensive coverage of the potential drivers of change in environmental strategy and MCS in a GCCP-focused context can be obtained. Transactional cost economics 
highlights how transactional characteristics influence efficiency-driven strategy and MCS change. Institutional theory emphasises the impact of GCCP-related societal and institutional factors on generators' discretion in changing their environmental strategies and MCS. Resource-based theory examines how the choice of appropriate environmental strategy and MCS is constrained, or enabled, by the generators' internal resources and capabilities. Further, the combined use of TCE, RBT and IT provides an opportunity to examine the relative importance of these economic and institutional drivers in environmental strategy and MCS change decisions throughout a longitudinal period of GCCP change.

Fourthly, using the three theories in conjunction provides an opportunity for theory triangulation. Triangulating the results between TCE, IT and RBT can explain the impact of GCCP-related volatilities and pressures on each generator. Consequently, an assessment can be made as to whether such volatilities and pressures influence internal efficiency, social legitimacy or market competitiveness of different generators. Theoretical triangulation also enables the identification of the primary motivations for each generator's change in environmental strategies. The use of the three theories helps identify the key objectives underlying GCCP-related MCS design and use and explains why such objectives may vary across generators, e.g. why some generators primarily use MCS to focus on enhancing competitiveness while others select MCS mostly to optimise internal efficiency. Therefore, integrating the three theories allows the investigation of how generators seek to balance internal efficiency, social legitimacy and competitiveness needs through strategy and MCS choice (Westley and Vrendenburg, 1991; Hart, 1995; Deephouse, 1999). In doing so, an economic basis for explaining strategic and MCS change can be gained in addition to institutional theoryfocused insights.

In summary, the above sections argue that the three theories, TCE, IT and RBT, are inter-related and compatible with each other. Additionally, their simultaneous use enables a comprehensive coverage of the economic and social factors in the GCCPrelated environments, and the assessment of such factors' impacts on the generators' environmental strategies and MCS. A basis for theory triangulation is also provided to assess the primary perceived impacts of the GCCPs on each generator and the key objectives underlying their GCCP-related strategy and MCS change. Based on TCE, IT and RBT, and the literature reviewed in Chapters 2 and 3, the next section outlines the research design and framework of this study. 


\subsection{Research Design and Framework}

This section first discusses the research design, which incorporates the constructs of the external and internal drivers of environmental strategies, the types of environmental strategies, and organisational MCS. Following the research design, a research framework is presented. Reference to the research gaps presented in Figure 3-5 are made to indicate how this study addresses this gap.

\subsubsection{GCCP-related external drivers}

In Chapter 2, it was argued that the planned GCCPs impose additional economic volatilities and societal pressures within generators' external environments. The external volatilities and pressures identified are policy and regulatory change, changing fuel economics, carbon market fluctuations, and increased societal pressures (Figure 2-4). These volatilities and pressures are consistent with the theoretical insights of TCE, IT and RBT discussed above. Institutional theory and TCE assist in explaining the impact of policy and regulatory change on environmental uncertainty, and the resulting strategic discretion available to organisations in terms of organisational learning and adaptation costs. Transactional cost economics also provides a basis for explaining the implications of changing fuel economics for both internal production costs and transactional costs relating to fuel monitoring and decision-making. Additionally, RBT suggests that changing fuel economics will modify the relative competitiveness between the generators. Further, carbon market fluctuations impose volatilities on carbon and transactional costs for thermal generators (a TCE perspective), and provide potential revenue increases for generators that are net sellers of carbon credits (an RBT perspective). Societal pressures can be viewed from either an IT or RBT viewpoint. While IT indicates that societal pressures affect the generators' social legitimacy, RBT suggests these pressures also affect the relative competitiveness of generators due to the variations in reputation associated with their generation asset and technology base.

Based on a review of the prior literature, Chapter 3 suggests further external factors, including environmental regulation, external economic uncertainty, industry-related societal pressures, stakeholder pressures, and field and peer pressures (Figure 3-5). These have all been found by previous studies to be significant drivers of change in environmental strategies or organisational MCS. The impacts of these external factors on strategy and MCS can be explained from a TCE or IT perspective. The introduction of environmental regulation imposes additional compliance costs (TCE) and/or increases the visibility of environmental issues and hence threatens an organisation's social legitimacy (IT). Environmental regulation may also place direct pressure on 
organisations with regard to adopting environmental strategies and MCS that are considered legitimate by regulators (IT). External economic uncertainty causes volatility in organisational costs (TCE) or market competitiveness (RBT). Such uncertainty is captured by the external factors discussed in the preceding paragraph; policy and regulatory change, changing fuel economics, and carbon market fluctuations. Additionally, organisations operating in some industries may experience stronger societal pressures and thus higher environment-related legitimacy threats than others due to the nature of their business (IT). In order to preserve their social legitimacy (IT), organisations need to also respond to stakeholder, field and peer pressures by changing their environmental strategies and MCS.

To ensure the comprehensiveness of analysis, this study combines the external factors identified in both Chapters 2 and 3 and discussed above in the research design. Therefore the following external drivers of environmental strategies will be examined: the GCCPs as environmental regulation, policy and regulatory change, changing fuel economics, carbon market fluctuations, increased societal pressures (containing industry-related societal pressures ${ }^{3}$ ), field and peer pressures and stakeholder pressures. The examination of these external drivers provides an insight into changes in generators' environmental strategies in a GCCP context (Gap 1). Furthermore, the impacts of GCCP changes on generators' economic and social environments can be assessed (Gap 3a, Figure 3-1).

\subsubsection{GCCP-related internal characteristics and capabilities}

Insights from Chapters 2 and 3 and from the past research application of TCE, IT and RBT are used to identify the internal factors that potentially influence generators' environmental strategies and MCS choice in a GCCP-focused context. Chapter 2 argues that the emission profile associated with asset and technology base is likely to drive each generator's carbon and compliance costs (Figure 2-4), consistent with the TCE predictions. The impact of an emission profile on environmental strategies can also be viewed from an IT perspective. For example, generators with a higher emission profile will be exposed to greater societal criticisms, thereby threatening their social legitimacy. Thus, they are more likely to change their environmental strategies and associated MCS to manage emissions.

\footnotetext{
${ }^{3}$ Since this study examines organisations operating in a single industry, there are no inter-industry differences in societal pressures. Thus, societal pressures by default include public pressures exerted on all the studied organisations due to the nature of their industry (electricity).
} 
Furthermore, based on TCE, this study argues that transactional characteristics determine the level of transactional costs, which in turn require the appropriate environmental strategies and MCS to manage them. Consequently, three transactional characteristics will be examined: asset specificity; transactional frequency; and transactional uncertainty (Section 4.3.1). The transactions analysed will be those relating to the measurement, monitoring, reporting, and verifying of emissions, and the buying and selling of carbon credits to satisfy GCCP obligations, or to generate revenue.

The literature in Chapter 3 further suggests that organisational size, technological and financial capabilities, managerial factors, prior experience and organisational learning, and organisational culture (Figure 3-1) are the internal capabilities and characteristics that drive environmental strategies. Except for organisational size, all the other factors can be explained from an RBT perspective, as organisations can utilise particular combinations of these internal capabilities to pursue particular environmental strategies and gain a competitive advantage. Organisational size can be viewed from either an IT or RBT perspective. Institutional theory suggests that the larger a generator is, the more likely it is to be exposed to political and public pressures to demonstrate its environmental responsibility. Alternatively, RBT suggests that larger organisations have the financial and human resources available to pursue the extensive and wider scope environmental practices that are required to manage the organisational impacts of GCCPs.

In combination, this study will examine the following potential internal drivers of organisational environmental strategies and MCS choice: emission profile; transactional characteristics; organisational size; technological and financial capabilities; managerial factors; prior experience and organisational learning; and organisational culture. The inclusion of these internal drivers provides an insight into their impacts on organisational decisions to change their environmental strategies and MCS in response to GCCP changes. Furthermore, the potential interaction and inter-relationships between internal and external drivers in driving environmental strategic changes can be examined (Gap 3b, Figure 3-1).

\subsubsection{Impacts of external and internal drivers on the generators}

As discussed, the above external and internal drivers are likely to have significant impacts on the generators which in turn trigger strategic and MCS change. These impacts are reflected in the implications for organisational carbon and transactional costs, market competitiveness and social legitimacy. Organisational costs, the 
management of which is the basis for internal efficiency, are influenced by external drivers including policy and regulatory change, changing fuel economics, and carbon market fluctuations (TCE). Internal drivers including emission profile and transactional characteristics also affect organisational costs (TCE). Understanding these impacts of external and internal drivers on costs provides an economic basis for explaining strategic and MCS change (Gap 2, Figure 3-1). Additionally, generators' market competitiveness is likely to be affected by external drivers including changing fuel economics and carbon market fluctuations, and societal pressures. The internal capabilities identified in Section 4.4.3 (organisational size, technological and financial capabilities, and managerial factors) also enable generators to gain a competitive advantage (RBT). External institutional drivers including increased societal pressures, field and peer pressures, and stakeholder pressures, have implications for generators' social legitimacy (IT). Further, organisational size and a high emission profile expose generators to strong societal pressures and legitimacy threats (IT). Thus, not only the influence of individual drivers on generators can be assessed, but the potential combined impact and relationships between external and internal drivers in determining these impacts, can also be identified (Gap 3b, Figure 3-1).

\subsubsection{Change in environmental strategies}

To address the impacts of GCCP-related external and internal drivers on organisational costs, market competitiveness and/or social legitimacy, generators will need to change their environmental strategies. The degree of these impacts will drive the extent that generators change their environmental strategies. For example, a generator exposed to high potential competitiveness loss due to GCCP-related changing fuel economics is more likely to change their environmental strategies significantly. Similarly, a generator having a high degree of internal capabilities, such as renewable expertise and assets, and hence a strong potential to enhance GCCP-related competitiveness is more likely to make substantial changes to its environmental strategies. Additionally, the combined impact of external and internal drivers on the extent of environmental strategic change can be determined (Gap 3c, Figure 3-1). For example, an assessment can be made concerning whether a generator, being exposed to positive environmental conditions and having supporting internal capabilities, modifies its environmental strategies more extensively.

As reviewed in Section 3.4, previous studies suggest a variety of ways to classify environmental strategies, but these classifications are significantly overlapping. Overall, environmental strategic changes/responses vary along a continuum from no response, to 
reactive, and to proactive, and thus organisations pursue differing levels of proactiveness in responding to environmental issues and regulations. Therefore, the first dimension of change in environmental strategy examined in this study is the level of strategy proactiveness. For the purpose of this study, the level of strategy proactiveness is represented by the scope of the initiatives undertaken as part of environmental strategies, the number of organisational functions involved in implementing such initiatives and the amount of financial provision made for them, and the extent such initiatives are undertaken prior to the announcement and/or introduction of a particular GCCP. Thus, strategy proactiveness determines how early generators establish or change their environmental strategies, and how broad such strategic changes are.

Alternatively, in response to the economic and institutional impacts associated with GCCP changes, generators may not adopt new environmental strategies. Instead, they may choose to place different degrees of strategic emphasis on existing strategies. For example, the implementation of an emissions management strategy prior to the ETS announcement may represent an operational concern. However, after the announcement, this strategy may be assigned strategic importance because its effective implementation is critical to the mitigation of potential ETS compliance costs. Therefore, strategic emphasis is the second dimension of change employed in examining generators' environmental strategies. For the purpose of this study, strategic emphasis is determined by the level of attention that top management places on a particular environmental strategy. Such attention is reflected in the amount of information required and discussed at top management levels regarding the performance of an environmental strategy and its associated initiatives.

Consequently, this study examines strategy proactiveness and strategic emphasis as the two dimensions for the change in environmental change made by generators in response to the GCCPs. Doing this enables longitudinal insights into how generators' environmental strategies are modified throughout a prolonged period of GCCP change (Gap 3d, Figure 3-1).

\subsubsection{Change in organisational MCS}

To implement change in environmental strategies, generators need to modify their organisational MCS. The literature suggests that an organisation's MCS is driven by the choice of its strategies. However, no prior study could be identified that has empirically examined the impact of environmental strategy proactiveness on MCS design, use and objectives. Since strategy proactiveness concerns the scope of initiatives and the number of functions involved in strategy implementation, this study argues that more proactive 
environmental strategies require the selection and use of a higher number of and more broader-scoped controls and control systems. Additionally, consistent with prior studies (Simons, 1990, 1991; Abernethy and Brownell, 1999), strategic emphasis can be argued to drive MCS use. Environmental strategies with a strategic emphasis will require an interactive use of selected controls and control systems. An interactive use enables top management engagement and supervision and thus ensures that such strategies are effectively planned and implemented. The analysis of the impact of environmental strategy proactiveness and strategic emphasis on organisational MCS provides an enhanced insight into the role of MCS within the strategy implementation and planning process (Gap 4, Figure 3-5).

MCS is defined in this study as:

a system of organisational information seeking and gathering, accountability and feedback designed to ensure that the enterprise adapts to changes in its substantive environment and that the work behaviour of its employees is measured by reference to a set of operational sub-goals (which conform to overall objectives) so that the discrepancy between the two can be reconciled and corrected for. (Lowe, 1971, p. 5)

This definition is adopted because it provides a holistic view of organisational MCS and focuses particularly on organisational adaptation to a changing environment (Kloot, 1997). It captures both strategic issues, i.e. the interaction between the organisation and its environments, and the formulation of strategic plans, and operational issues (the implementation of strategic plans). The definition also emphasises the need for synergy between the organisation and its environments. It further highlights that MCS are designed and used to influence behaviour to ensure cooperation and goal congruence for the achievement of organisational objectives (Flamholtz, Das and Tsui, 1985).

For the purpose of this study, MCS comprises both controls and control systems. Controls include formal controls such as rules, standards, operating procedures and budgets, and informal controls, which emerge from, or reflect organisational culture (Anthony, 1965; Ouchi, 1979). Control systems are information systems which measure, monitor, and report external and internal information and provide such information to managers to assist their decision-making. Control systems also include communication and feedback systems to enable coordination, organisational learning and change (Otley, 1999). The collection of controls and control systems and their use by managers to ensure the adaptation of organisations to the environments and the 
achievement of organisational objectives constitutes the organisational MCS (Otley, Broadbent and Berry, 1995).

Three aspects of organisational MCS is examined in this study; design, use, and objectives. In terms of MCS design, the MCS literature suggests that consideration of: i) informational scope and budgetary controls; ii) environmental performance management system and environmental accounting; and iii) communication and coordination systems, are required within an MCS to support environmental strategies (Section 3.5). However, this list of controls and control systems is limited and may not capture the full range of MCS components that generators can adopt in relation to their environmental strategies. Thus, to provide a more comprehensive understanding of generators' MCS design, this study utilises Simons' (1991) four broad types of MCS components: belief systems; boundary controls; diagnostic control systems; and interactive control systems (Section 3.5.2).

To capture MCS use, this study examines the degree of use of particular controls and control systems, or MCS component types, by management level and frequency of use. Thus, a higher degree of use indicates regular use of a particular control, control system, or MCS type at a top management level. More detail on how MCS use is ranked in this study is provided in Chapter 5 (5.7.2.4).

Additionally, the prior literature suggests that organisational MCS serves different distinct objectives, namely: ensuring internal efficiency; enhancing market competitiveness; managing external uncertainty; legitimising or rationalising; and serving political purposes (Section 3.5.3). These objectives will be examined as the third and last aspect of generators' MCS.

\subsubsection{Proposed research framework}

A research framework, based on the components/constructs identified above and the potential relationships between the GCCPs, external and internal drivers, environmental strategies and MCS, is developed. This framework (Figure 4-2) integrates the factors found in the literature reviews in Chapters 2 and 3, and theoretical insights from TCE, IT and RBT as discussed in this chapter, in order to examine the implications for organisational MCS due to the strategic changes generators make in response to GCCPrelated volatilities and pressures. 


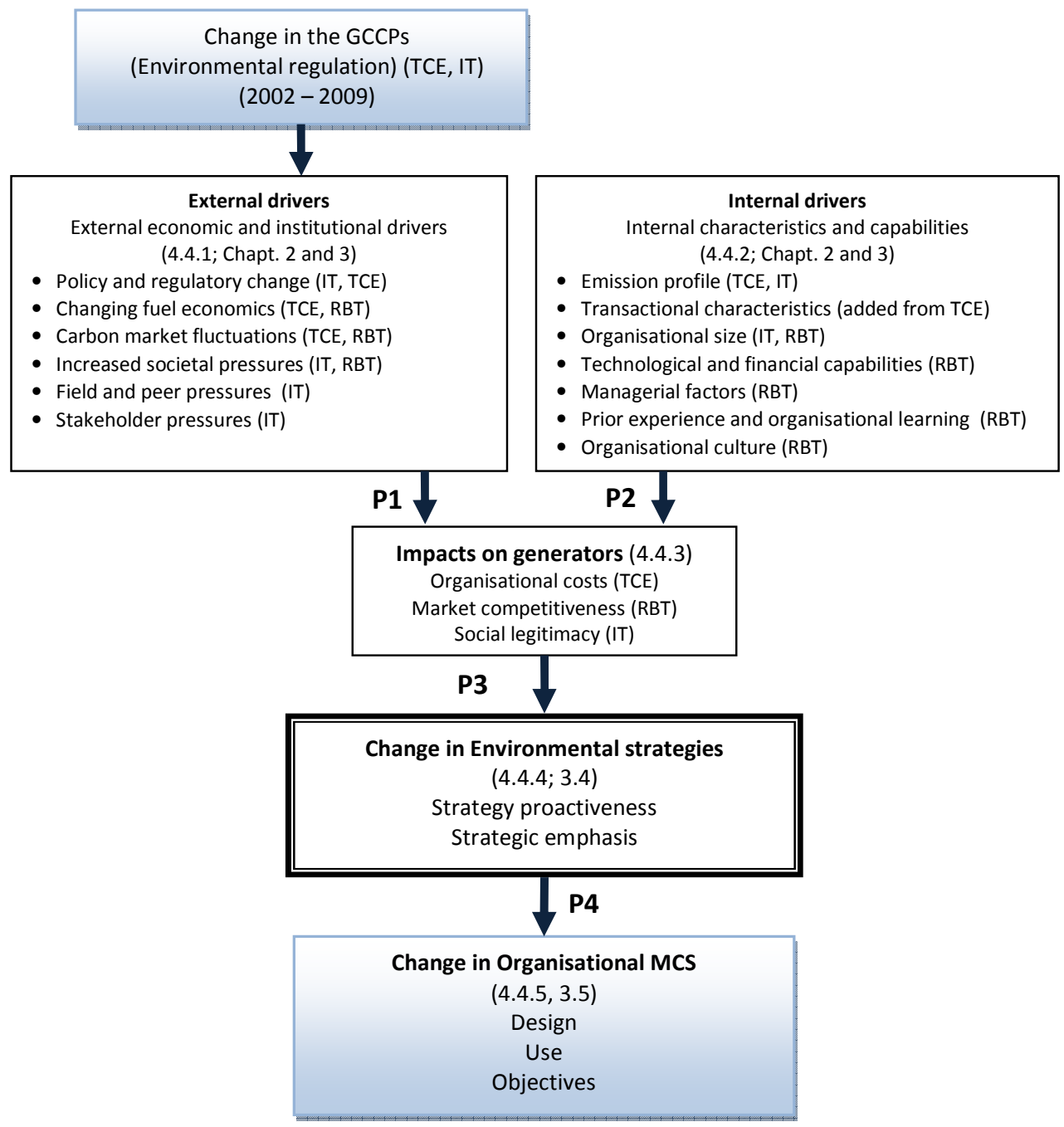

Note: Arrows do not indicate causal relationships

From this framework and the discussion above, four broad propositions are identified to provide a basis for this study's empirical analysis:

P1: External GCCP-related economic and institutional drivers impact on generators' competitiveness, costs and legitimacy;

P2: Internal GCCP-related characteristics and capabilities impact on generators' competitiveness, costs and legitimacy;

P3: The impacts of GCCP-related external and internal drivers in turn lead to a change in environmental strategies; and

P4: The change in environmental strategies requires corresponding modifications in organisational MCS. 
Assessing these four propositions will enable the research question to be addressed. This allows the identification of the implications for organisational MCS arising from strategic choices the New Zealand electricity generators make in managing GCCPrelated economic and social volatility and pressures resulting from a prolonged period of GCCP change.

\subsection{Summary}

This chapter has developed a research framework that will be used to guide the process of data collection and analysis. The framework is built from the theoretical insights suggested by TCE, IT and RBT as well as findings from prior literature. The framework and associated propositions argue that GCCP changes influence generators' external environments, which in turn affects organisational costs, market competitiveness, and social legitimacy. Additionally, generators' internal characteristics and capabilities have the potential to influence their organisational costs, competitiveness and legitimacy in a GCCP context. In response to these externally and internally-driven impacts, generators will need to change their environmental strategies, which are operationalised through two dimensions; strategy proactiveness and strategic emphasis. The change in generators' environmental strategies in turn will have different implications for MCS design, use and objectives. The next chapter outlines the research methodology and discusses the tools and techniques used in data collection and analysis. 


\section{Chapter 5: Research methodology}

\subsection{Introduction}

Chapter 2 outlined how this study examines the implications for organisational MCS arising from the strategy choices electricity generators make in response to the volatility and pressures associated with GCCPs. The study period was from 2000 to 2009 during which several GCCPs were planned and changed. To guide this study, Chapter 4 developed a research framework and four propositions relating to generators' strategic and MCS change in a GCCP-focused context. This chapter identifies the data types required to assess the framework and resultant propositions and explains the tools and techniques that will be used to collect and analyse those data.

This chapter is organised as follows. The next section justifies the main methodology employed in this study, a longitudinal multiple-case study approach. Then, the choice of cases and study period is explained. The following two sections discuss the use of reflection and triangulation methods in order to increase the rigour and reliability of this study's results. In the next two sections, specific techniques for data collection and data analysis are outlined. The last section summarises the chapter.

\subsection{A longitudinal multiple-case study approach (LMC)}

\subsubsection{An overview of a case study approach}

This study positions itself in a case study research tradition. A case study approach is defined as an empirical inquiry that investigates a contemporary phenomenon within its real-life context and in which multiple sources of evidence are used (Yin, 2003). The adoption of a case study approach allows accounting practices, such as MCS, to be investigated within the organisational context in which they operate (Hofstede, 1968; Hopwood, 1972; Otley, 1978). Further, a case study approach is appropriate because this study examines a contemporary event, the planned GCCPs, which have prolonged impacts on generators (Yin, 2003). A case study approach can also reveal rich detail about the complexity and dynamics of organisational strategies, processes, and characteristics (Lillis and Mundy, 2005). In particular, a multiple-case analysis enables the researcher to understand the impacts of important field- and industry-level contextual factors on an organisation's strategies and MCS over time (Hopper, Otley and Scapens, 2001). 
The qualitative research method used in this study is a longitudinal multiple-case study (LMC) approach (Yin, 2003; Lillis and Mundy, 2005). Accordingly, it involves a tenyear study (from 2000 to 2009) of strategic and MCS changes within multiple cases in a single industry, i.e. five generators within the NZEI. This approach has less depth but more breadth than a single case study approach (Yin, 2003).

\subsubsection{The applicability and benefits of LMC to this study}

An LMC approach is adopted in this study for three reasons. First, since the research question focuses on MCS implications throughout a prolonged period of GCCP change, a longitudinal approach is required. Second, a single case study is not appropriate because there are five generators in the NZEI with different organisational histories and characteristics and hence potentially varying strategic and MCS responses to GCCP change. Third, these five generators represent more than $90 \%$ of the industry in generation and sales activity (Section 2.3.2).

A LMC approach has a number of advantages over a single in-depth case study and cross-sectional survey approaches. Although an in-depth single case study can provide rich details on the internal organisational factors and mechanisms, it cannot provide cross-case patterns that potentially enhance the external and internal validity of the study (Lillis and Mundy, 2005). Further, although in-depth single case study places strong importance on the historical, cultural and situational nature of organisational strategies and MCS, it has limited generalisability to other organisations and industries due to the single-case examination (Armstrong, 1985; Miller, 1998; Wallander, 1999). Therefore, a single case study is not appropriate for this study which examines change in environmental strategies and MCS within multiple generators which have different organisational characteristics (2.3.2).

In contrast, a cross-sectional survey approach provides patterns of relationships between the investigated factors and insights into the impacts of external factors such as industry and regulation. However, it allows research only at a particular point in time and cannot provide contextual explanations due to multiple respondents (Lillis and Mundy, 2005). Since a cross-sectional survey does not capture the impact of change over time, it is not suitable for this study which investigates implications for organisational MCS over a prolonged period.

The LMC approach overcomes the limitations of the single-case and cross-sectional survey approaches. It allows some depth of insight into the internal mechanisms and factors influencing the choice of environmental strategies and associated organisational 
MCS. Further, the LMC approach enables the exploration of the impact of external and contextual factors on such choices and how such impact changes over time (Hopper, Otley, and Scapens, 2001). It also provides a longitudinal understanding of MCS change in multiple cases (i.e. electricity generators) throughout a ten-year period (Yin, 2003; Lillis and Mundy, 2005). By adopting a LMC approach, this study has less depth than a single case study but more breadth due to its multi-case analysis. In contrast, it has more depth but is more limited in breadth than a broad-based survey (Lillis and Mundy, 2005). The trade-off between depth and breadth is a conscious choice based on a judgment of the LMC approach's appropriateness for this study's research question.

\subsubsection{Mitigating the potential limitations of qualitative research}

Despite its benefits, the case study approach as a method of qualitative research is often criticised as having limited generalisability, validity, and reliability due to a failure to follow research protocols originally designed within an economics-based quantitativefocused paradigm (Zimmerman, 2001; Ahrens and Chapman, 2006). In response to these criticisms, scholars suggest that case study researchers need to demonstrate they have been rigorous and unbiased in their research by employing verification strategies throughout the enquiry process (Ahrens and Dent, 1998; Morse et al., 2002; Lillis, 2006).

By adopting the LMC approach, this study mitigates the potential limitations of qualitative research. First, through the choice of multiple cases that represent the population (NZEI), this study achieves a degree of generalisability and external validity (Lillis and Mundy, 2005). To add further rigour to the research process and findings, this study adopts a number of verification strategies as suggested by Morse et al. (2002). These strategies include replication logic in choosing cases (Section 5.3.1), concurrent data collection and analysis (as part of reflection strategies in Section 5.4), and audit trails (Section 5.7.1). Additionally, results are verified using data and theoretical triangulation (Section 5.5).

Consistent with the LMC approach, this study examines the environmental strategies and MCS of five generators in the NZEI over a ten-year period of GCCP change. The next section justifies and discusses, in more detail, the choice of cases and study period. 


\subsection{Choice of cases and study period}

\subsubsection{Choice of cases}

Prior research (Arnold, 1970; Yin, 2003; Lillis and Mundy, 2005) suggests that a multiple-case study approach should employ replication logic in choosing its cases. Replication logic involves a non-random and purposive choice of cases to reflect different outcomes argued by theory (Yin, 2003). Further, researchers are advised to choose cases with contrasting organisational-level characteristics (Eckstein, 1975; Hammersley, 1992; Yin, 2003) as it helps validate the chosen theories and any associated propositions, such as those outlined in Chapter 4.

Accordingly, this study will choose cases based on their differences in key organisational-level variables so as to maximise case diversity (Merchant, 1985). Consequently, all the five major generators mentioned in Chapter 2 are selected. As outlined in Chapter 2, these generators are major generators and retailers in the NZEI. In aggregate, they make up $91 \%$ and $95 \%$ of New Zealand wholesale and retail electricity market share respectively. This choice of cases ensures that almost the whole generating and retailing sectors are covered. In doing so, it minimises the potential for selection bias and optimises the assessment of the validity of the research framework. The other generators and retailers in the NZEI generating and retail sectors are significantly smaller in size and are not vertically integrated. Therefore, by focusing on the largest five generators, this study controls for the impact of industry and the nature of the organisation's business on environmental strategies and MCS.

To maintain interview confidentiality, these generators are referred to as Firm A, B, C, $\mathrm{D}$ and $\mathrm{E}$. These generators differ from each other across a number of dimensions, including generation capacity, ownership structure, and the level of wholesale activity. The differing organisational characteristics potentially lead to variations in the external volatilities and pressures each generator perceives in relation to a GCCP, and thus have different implications for environmental strategies and organisational MCS. Therefore, an assessment can be made regarding the drivers of the similarities and differences among these generators in regards to the environmental strategic changes they make in a GCCP-focused context. In turn, this assessment provides a basis for determining the potential impact on each generator's MCS. From this, general patterns relating to change in the generators' environmental strategies and MCS in response to GCCPs can be found, enabling the research question to be answered and the propositions assessed. 


\subsubsection{Choice of external stakeholders}

In order to supplement and support the multiple-case approach, this study also examines the perceptions and perspectives held by external stakeholders related to the electricity industry and GCCP processes and any pressures exerted by them on the generators. These external perceptions and perspectives will primarily be used to verify the data provided and/or generated by the generators and, secondarily, in some cases, to provide additional insights regarding the GCCPs and the strategic responses adopted by generators. Following replication logic (Merchant, 1985; Lillis and Mundy, 2005), this study will choose external stakeholders based on two dimensions:

- Variations in their involvement in generators' carbon-focused projects and strategy choice and change process;

- Variations in the role played by these organisations in relation to the GCCPs processes.

This study targets a number of key external stakeholder groups. Firstly, the Government, other political parties, regulators and government agencies, and an accounting professional body are chosen because they are the policy makers and standard setters whose actions and decisions represent coercive and regulatory pressures that organisations have to monitor and respond to (Scott, 2001; Fowler, 2009). Secondly, accounting and law firms and carbon trading specialists are important because organisations are likely to rely on their advice while evaluating the impacts of government policy and implementing environmental strategy and MCS changes in response (Abernethy and Chua, 1996; Scott, 2001).

Thirdly, industry associations, networks and lobby groups are targeted as they provide key mechanisms through which organisations voice their concerns regarding government policy (Masters and Keim, 1985; Hillman, Keim, and Schuler, 2004). These groups also provide a forum for knowledge dissemination among organisations, which potentially influences the organisational strategic and MCS responses (Barreto, BadenFuller and de Cima, 2006). Fourthly, this study is interested in the opinions of key journalists (e.g. who write on the electricity industry and GCCP-related topics) because their articles represent direct media pressure on organisations to alter their environmental strategies (and associated MCS) to maintain organisational reputation and social legitimacy (Sharma and Nguan, 1999; Bansal, 2005). Finally, major research institutes are included because their research findings potentially influence government 
policy design, and help organisations better understand their exposure under a given policy (e.g. a GCCP) and to formulate their strategies accordingly.

Forty external stakeholders were identified as being the most active in relation to GCCP processes and the generators' potential environmental strategies. All forty organisations were contacted and asked to participate in the study. Twenty eight agreed, representing a $70 \%$ response rate.

\subsubsection{Choice of study period}

To gain a longitudinal view of organisational responses in relation to change in GCCPs, this study collects and analyses interview and documentary data for a ten-year period from January 2000 to October 2009. Chapter 2 (Figure 2-2) outlines the key events and changes in the GCCPs over this period. This period is further separated into five periods, around transition points that determine GCCP change (Phillips, 2002):

- From January 2000 to March 2002 before any GCCPs were announced (to allow for identification of changes in environmental strategies and MCS prior to, and post, any GCCP proposal);

- From April 2002 when the planned carbon tax was announced to December 2005 when the plan was cancelled;

- From January 2006 to September 2007 when there was no explicit GCCP but the draft NZES indicated a preference for an ETS;

- From October 2007 when the Original ETS was announced until September 2008 when the Climate Change Act (2008) was passed;

- From October 2008 when there was a change of government which led to a select committee review of the Original ETS and proposed legislative changes to the ETS, up until October 2009 when the Moderated ETS Bill was introduced.

Data are analysed based on the periods outlined above. Accordingly, evidence regarding the environmental strategies and MCS employed will be presented in a chronological order (i.e. during relevant periods) so that strategic and MCS changes over time in response to the planned GCCPs within each generator can be identified (Chapter 6). The results are then analysed across the five generators to gain longitudinal and comparative insights into GCCP-related external and internal drivers and change in environmental strategies (Chapter 7) and change in organisational MCS (Chapter 8). 
In order to enhance the rigour of the research process and the richness of this study's results, reflection and triangulation methods are employed. Each of these is discussed below.

\subsection{Reflection methods}

Reflection is a flexible and ongoing process in which different stages of the qualitative enquiry, including defining the research problem, choosing (an) appropriate theory(ies), and collecting and analysing data interact and influence each other so as to find a possible fit between the research problem, theory and data (Ahrens and Chapman, 2006). Reflection is employed in this study not only within the processes of data collection and analysis, but also to establish and check the linkages between these and the research framework.

This study adopts an interactive pattern in its research process where the preliminary findings inform decisions about future data collection (Covaleski and Dirsmith, 1988; Miller et al., 2004). Data collection is divided into three phases to enable the reflectivity and interaction between data collection and other steps in the research process. The first phase is where data are collected to gain a general understanding of the field and to help inform the identification of the research question. This phase informs the research context and the establishment of the research question as highlighted in Chapter 2. The second phase focuses upon collecting data related to one generator (Firm A) and changes in its environmental strategies and implications for organisational MCS in the five time periods outlined above. Data from this phase are analysed to discern key trends and patterns, and to evaluate their correspondence to the proposed theories and research framework (Covaleski and Dirsmith, 1990; Ahrens and Chapman, 2006). The

analysis of Firm A's evidence provides insights into the most significant or interesting issues for field participants, which are then used to revise later interview questions and the choice of archival documents (Ahrens and Dent, 1998). The revised interview questions and document choice are applied to the third and final stage, where data relating to the remaining four generators (Firms B, C, D and E) and external stakeholders are collected and analysed.

Reflection is also utilised within each step of data collection. Specifically, within each case, opinions and perspectives gained from earlier interviews and archival data are directly communicated to later interviewees who are asked to comment and reflect on those opinions/perceptions from their own organisational perspectives (Covaleski and Dirsmith, 1988). The purpose of this reflection strategy is twofold. Firstly, direct 
reflection is used to cross-check and validate ideas and tentative findings from earlier data collection/analysis (Covaleski and Dirsmith, 1988). Secondly, comparing and validating internal versus external participants' views provides an assessment of the validity of different theories in explaining environmental strategies and MCS change (theory triangulation, Section 4.3.6) (Ahrens and Chapman, 2006).

Consequently, the use of reflection methods potentially enables research framework validation, verifies the research findings, and provides additional insights and richness to the understanding of environmental strategies and MCS under a GCCP-related context (Covaleski and Dirsmith, 1990; Ahrens and Dent, 1998). Another method, triangulation, is also used to increase the validity and reliability of this study's results, as discussed next.

\subsection{Triangulation methods}

Triangulation is defined by Flick, Kardorff and Steinke (2004) as 'observation of research from (at least) two different points' (p.178). The primary benefits of triangulation are in validating the findings and increasing the richness of descriptions and insights about the subjects under study (Ahrens and Chapman, 2006; Denzin, 2009). Any single method or theory can only explain one aspect of empirical reality and therefore it cannot rule out rival explanations (Denzin, 2009). Triangulation enables the assessment of these rival explanations and thus can increase the validity of interpretation (Bourgeois and Eisenhardt, 1988; Marginson, 1999). Additionally, triangulation can capture a more holistic and contextual picture of the units under study (Jick, 1979).

Triangulating findings gained across different methods, data, theories or researchers can reveal additional insights that may be neglected by a single method, data source, theory or researcher (Denzin and Lincoln, 2003). Hence, there are four types of triangulation: methodological triangulation, researcher triangulation, theoretical triangulation, and data triangulation (Denzin and Lincoln, 2003; Denzin, 2009). These types of triangulation can be used separately or in conjunction with each other. Denzin (2009) suggests that 'multiple strategies of triangulation are proposed to be the preferred line of action' (p.313) Hence, multiple methods, theories and data sources and researchers can be combined in one study to enhance the credibility of the explanations and to better capture the richness and complexity of the social phenomena (Denzin, 2009). 
Methodological triangulation is the combination of multiple methods in a study, and involves 'a complex process of playing each method off against the other so as to maximise the validity of field efforts' (Denzin, 2009, p.304). Since this study follows a case study approach, methodological triangulation is not employed. Researcher triangulation involves two or more researchers who are directly involved in collecting and interpreting data. The purpose of this is to limit, reduce and balance out the potential subjectivity of an individual investigator (Flick et al., 2004). Researcher triangulation is not adopted as the $\mathrm{PhD}$ dissertation is sole authored by necessity.

Theoretical triangulation refers to the use of multiple theories and perspectives in an empirical analysis (Flick, 1992). Using this method, new and additional meanings and definitions attached to the events, actions, or behaviour can be identified, and thus a more holistic understanding of the social phenomena under study can be gained (Denzin, 2009, p.227). This study employs theoretical triangulation between three theories, TCE, RBT and IT, as explained in Chapter 4 (Section 4.3.6).

Data triangulation draws on data from different sources at different times, from different places and from different people (Flick, 1992). This is first to discern consistent patterns and instances across different data sets and to confirm the validity or trustworthiness of a particular explanation, theory, or argument. The second use of data triangulation is to provide a richer and more in-depth story about a particular event, people, context or community (Flick et al., 2004). Data triangulation is operationalised in this study by drawing upon multiple internal and external sources of data and comparing the results between these sources.

The primary sources of data to be collected are interviews, archival and electronic documents from the New Zealand generators and relevant external organisations. Interviews are conducted with internal managers of these generators and representatives from external stakeholders to gain both internal and external views about the GCCPrelated changes made by the generators. The insights of internal and external participants are then compared and contrasted for cross-validation purposes.

Documents produced by the generators and by external stakeholders are also collected. Generator-related documents include annual reports, environmental/sustainability reports and media releases which are publicly available. Externally-generated documents including news and media coverage relating to generators, and financial reviews by the relevant government agencies or ministers are also obtained. These internal and external documentary data sets are compared to identify similarities and 
differences that help verify results and validate the research framework (Miller et al., 2004).

The interview data and documentary data are then triangulated against each other. Documents can confirm and/or disprove interview results (Ferreira and Merchant, 1992; Yin, 2003). Thus, convergence or divergence in the results between different data sources enables an assessment of the research framework's validity and increases the confidence in the results (Hopper and Hoque, 2006). The next section describes the data sources and methods of data collection used in the data triangulation.

\subsection{Data collection}

As mentioned, the two primary sources of data used are interviews and documents. Reliance on interview data supplemented by extensive analysis of documents is a popular and recommended practice in case study research (Yin, 2003). Specific methods employed to recruit interviewees and conduct interviews, as well as methods used to select and collect archival and electronic documents are discussed in this section. This is followed by the presentation of the data demographics.

\subsubsection{Methods of data collection}

\subsubsection{Interviews}

This study identifies potential interviewees by scoping the groups of respondents that are relevant to the study area/ context. The scoping is done by reviewing prior literature and then performing a preliminary analysis of publicly available documents related to the NZEI, and topics of climate change, sustainability and emissions trading. The following groups are identified as relevant: five generators (5.3.1) and forty external stakeholders (5.3.1). About half of these targeted organisations are approached using personal connections and the snow-balling technique (Arksey and Knight, 2002; Flick, 2009). Personal contacts are used to ensure that the person who is contacted will be willing to talk to the researcher (Arksey and Knight, 2002). The snow-balling technique is where the initial respondent subsequent to the interview will suggest further contacts, personally introduce the researcher to them or just allow their names to be mentioned in invitation letters sent to these contacts. The remaining half are contacted based on emails which include an invitation letter and a brief research proposal (Appendix A). The letter is customised so that it appeals to the person contacted. These strategies provide access to the organisations and individuals that are the most relevant to the study (Weiss, 1994; Warren, 2002). 
The study adopts a semi-structured method in conducting the interviews (Yin, 2003; Denzin, 2009). In semi-structured interviews, questions are specified, but the interviewee is free to move beyond these questions to elaborate on their own experiences and perceptions regarding a particular issue or past event relevant to the questions (Schram and Steen, 2001). Therefore, a semi-structured interview method allows more flexibility for the interviewees, as opposed to the standardised answers permitted by the structured interviews (May, 2001). This method also allows the interviewer to understand the interview context, which, in itself, is an important source of information to explain the statements made and views expressed by the interviewee (May, 2001).

The interviews were conducted in an informal manner and interviewees were given opportunities to focus on issues that interested them most, rather than being pressured to comment on every single item (Warren, 2002). For this reason, in most cases, there were common questions that were presented to all groups and customised questions that were only intended for one particular group. Customised questions were designed to fit with the specific roles, functions and areas of interest in relation to the generators and the GCCP processes. The questions were initially formulated based on the prior literature, with the aim to understand the factors and relationships captured in the four propositions. Then, following the reflection strategies described in Section 5.4, the questions are continuously updated to reflect new understanding and insights gained from the analysis of prior interviews. A list of sample semi-structured interview questions is provided in Appendix B.

\subsubsection{Documents}

As discussed in Section 5.5, this study uses data triangulation and thus collects documents from a variety of sources for the period from January 2000 to October 2009. Most of the relevant documents are available electronically and were downloaded from the websites of the generators, external organisations, New Zealand Parliament and media companies. Other documents not available through electronic sources were acquired using techniques including attendance at public hearings, and making formal requests to relevant organisations and government authorities. However, the researcher did not have access to internal and/or confidential documents relating to the generators such as meeting minutes and memos, due to the limited access allowed by top management of these generators.

Further, to gain insights into the changes in economic environments in which the generators operate, information on wholesale and retail market prices, emissions 
inventory of the electricity industry, and the economics of alternative fuels for electricity generation, were collected. To acquire an understanding of the regulatory and political environments, the documents obtained included the various reports issued by government agencies, statements made by ministers and officials, related parliamentary debate and the positions of different political parties on the planned GCCPs. Documents produced by external stakeholders, such as submissions to the planned GCCPs, discussion papers, reports, newsletters, media releases and public commentaries in relation to GCCPs and electricity-related issues, and disclosures relating to the involvement of generators in carbon-related projects were also collected. These documents provide an understanding of the wider social and political environments which were likely to influence generators' decisions to change their environmental strategies and MCS in a GCCP-focused context.

\subsubsection{Demographics of data collected}

\subsubsection{The five cases}

All five generators were invited to participate in this study and all agreed, with varying levels of access granted. In most cases, the researcher was introduced to one or two top managers or directors. The exception is Firm $\mathrm{C}$ in which the researcher was able to access both top and middle management levels.

As mentioned (5.3.1), the five generators examined differ from each other in a number of dimensions: generation bases; ownership structure; and the nature of their wholesale activity. The variations in these organisational characteristics between generators are summarised in Table 5-1.

Table 5-1: Variations in organisation-level characteristics of electricity generators

\begin{tabular}{|c|c|c|c|c|c|c|}
\hline \multirow[b]{2}{*}{ Cases } & \multicolumn{2}{|c|}{ Primary fuel source } & \multicolumn{2}{|c|}{ Ownership type } & \multicolumn{2}{|c|}{ Level of wholesale activity } \\
\hline & $\begin{array}{c}\text { Renewable- } \\
\text { based }\end{array}$ & $\begin{array}{c}\text { Thermal- } \\
\text { based }\end{array}$ & $\begin{array}{l}\text { State- } \\
\text { owned }\end{array}$ & $\begin{array}{c}\text { Privately- } \\
\text { owned }\end{array}$ & Net seller & Net buyer \\
\hline $\mathbf{A}$ & & $\checkmark(63 \%)$ & & $\checkmark$ & $\checkmark$ & \\
\hline $\mathbf{B}$ & & $\checkmark(67 \%)$ & $\checkmark$ & & $\checkmark$ & \\
\hline $\mathbf{C}$ & $\checkmark(100 \%)$ & & $\checkmark$ & & $\checkmark$ & \\
\hline D & $\checkmark(100 \%)$ & & & $\checkmark$ & & $\checkmark$ \\
\hline $\mathbf{E}$ & $\checkmark(87 \%)$ & & $\checkmark$ & & $\checkmark$ & \\
\hline
\end{tabular}

Two generators have more than 50\% thermal-based generation and are therefore labelled as thermal generators (A and B). The other three generators are predominantly renewable-based (more than $80 \%$ of generation capacity coming from renewable sources) and thus will be referred to as renewable generators (C, D and E). Three generators are state-owned $(\mathrm{B}, \mathrm{C}$ and $\mathrm{E})$ and the remaining two (A and $\mathrm{D})$ are privately 
owned and listed on the New Zealand stock exchange. Additionally, four generators have generation output exceeding their retailing commitments and thus they sell the rest on the wholesale market as net sellers. Only one generator (D) is a net buyer in the respect that its retailing commitment exceeds the electricity supplied from its generation assets, and thus has to purchase the rest of the required electricity from the wholesale market.

\subsubsection{Interviews}

Based on the agreement reached with the five generators, the researcher conducted thirteen interviews primarily with directors and senior managers. Interviewing at this organisational level has three benefits. First, these interviewees are the organisational policy makers and strategic planners and thus have the most knowledge about the impacts that the GCCPs will have on their organisations. Secondly, in understanding these impacts, directors and managers are likely to make decisions regarding strategy choices and associated MCS changes, required in response to the GCCPs. Thirdly, the senior managers interviewed are those with direct responsibility for ETS or carbonrelated strategies within these generators. Additionally, in Firm C, two middle managers directly involved in the implementation of GCCP-related strategies were interviewed.

Data collection took place from April to October 2009 when there was a high level of sensitivity and publicity about emissions and carbon trading issues due to the ETS review and potential ETS changes (Chapter 2). This sensitivity is the reason for the limited number of interviews that were able to be conducted with each generator. However, this limitation was offset by the extensive amount of publicly available documents and the interviews conducted with external stakeholders. Further, there is a difference in the number of interviewees participating from the five generators. Figure 5-1 provides a break-down of interviewees by generators. 


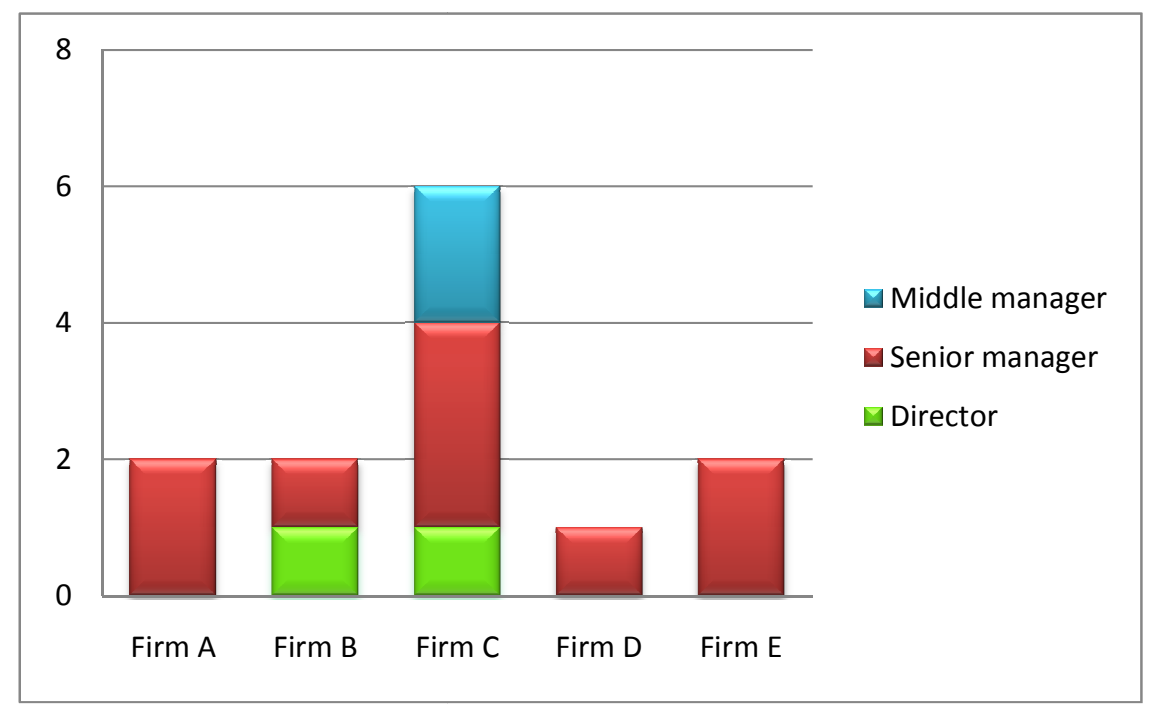

Additionally, 20 of the 40 external stakeholders were willing to be interviewed with one interview being conducted with each stakeholder. This involves a total of 32 interviewees because in some organisations two persons asked to join one interview. Most of these interviewees are either the chief executive or a director of these organisations. In the case of political parties, Members of Parliament representing each party or the senior advisors to the Members of Parliament were interviewed. An interview was also conducted with a journalist who writes regularly on generators and the GCCPs. A break-down of the interviewees by external stakeholders' target-groups is provided in Figure 5-2.

Figure 5-2: Break-down of interviewees by external stakeholders' target group

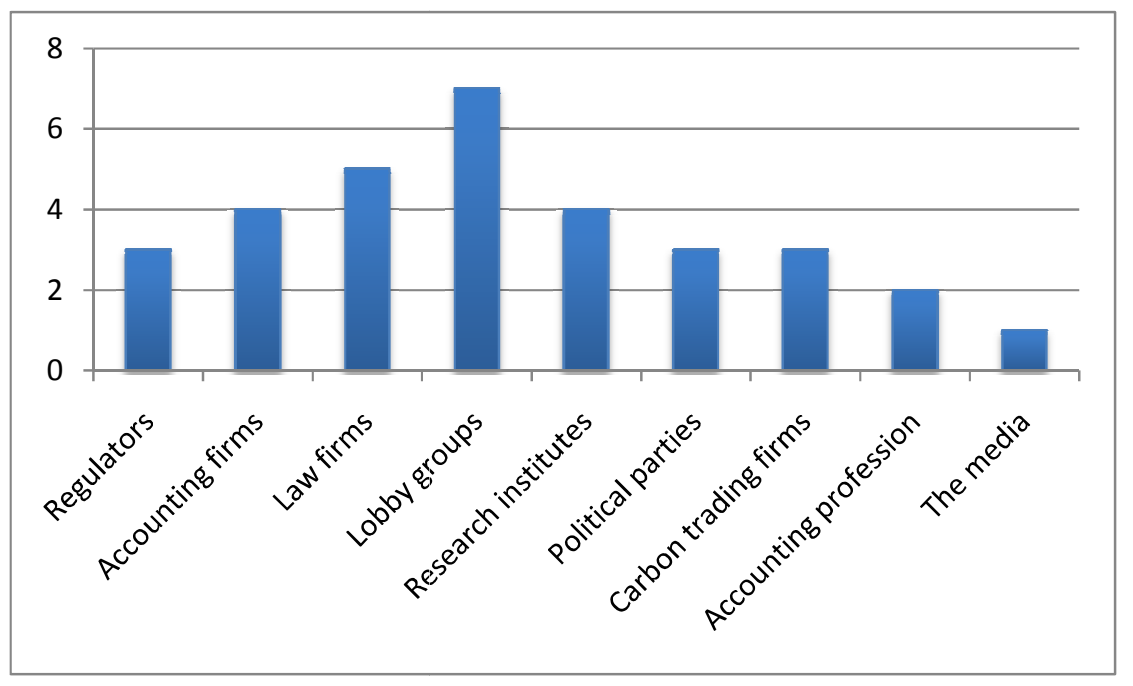

The interviews lasted from one to two and a half hours each and the time and location for the interviews were determined at the interviewee's convenience. All interviews

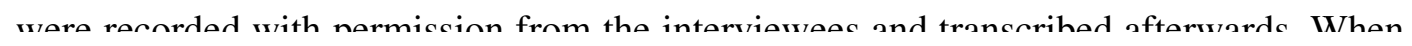


possible, these transcripts were sent back to the interviewees for the checking of accuracy in interpretation and expression. Investigator notes were also taken during and after the interviews. These notes were used as a source of information to collaborate the more detailed information in the interview transcript.

A list of semi-structured and open-ended questions was sent to the interviewee at least two days before the interview to allow time for preparation and thus more comprehensive answers. Most of the interviews were conducted on a one-to-one basis, except for some cases where two people were interviewed together so as to supplement each other's answers. However, it was very difficult to distinguish the differences in perceptions between the two participants since they tend to agree with each other's opinions. It is considered in these cases, that the collective opinions of the two interviewees represent the position of their organisation.

\subsubsection{Documents}

This study collected a total of 736 documents. A break-down of these documents is provided in Figure 5-3.

Figure 5-3: Breakdown of collected documents by target group

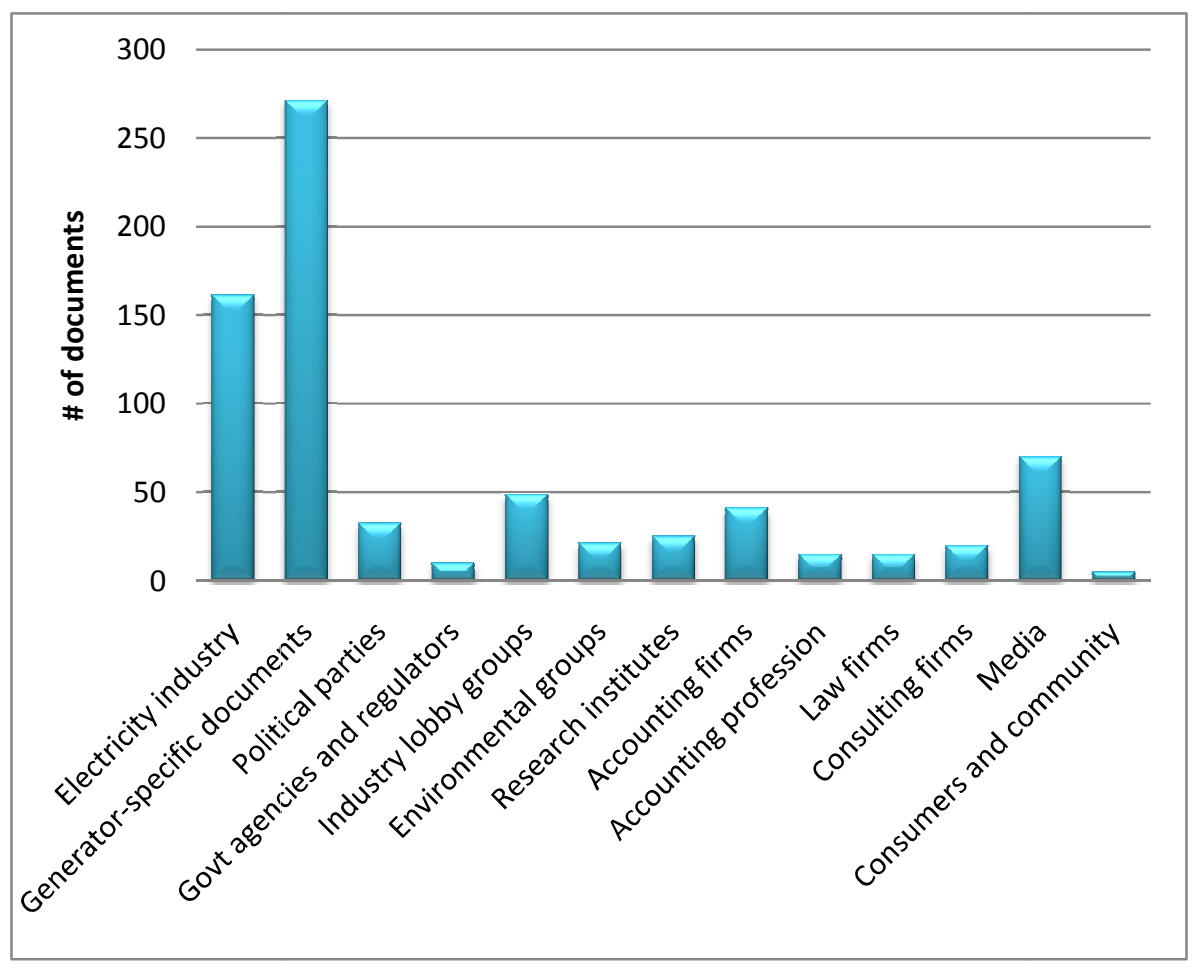

Documents related to the electricity industry (in general) accounted for $22 \%$ of the total documentary dataset and generator-specific documents, $36.8 \%$. The remaining $41.2 \%$ of documents were generated by external stakeholders, of which industry lobby groups, 
accounting firms, and the media represent the largest numbers of documents $(52.8 \%)$. This is attributable to two factors: i) the large number of organisations involved in each of these broad groups, and ii) the level of activity and responsiveness being displayed by these stakeholders during the debate around climate change and the ETS.

\subsection{Data analysis}

This study uses qualitative and quantitative techniques to interpret the collected interview and documentary data. To facilitate this interpretation, the study develops a coding system to classify the whole dataset into the categories (themes) relevant to the research question and propositions. This section discusses the procedures and techniques to be used for data coding and analysis.

\subsubsection{Data coding}

A coding tree is established in Nvivo8, to systematically identify and group recurrent patterns of particular instances, whether it is word, phrase, or large unit of some "meaning", across the data set (Neuendorf, 2002; Silverman, 2004). A qualitative coding software, such as Nvivo8, is valuable in enabling detailed analysis of text, as it enhances data retrieval and maintains an audit trail through data coding, summation and interpretation (Fielding and Lee, 1991). This audit trail can be replicated in other studies, which helps verify this study's results (Lincoln and Guba, 1985; Seal, 1999).

The instances used for coding were firstly developed based on existing theory and prior literature (Malina and Selto, 2001). These instances relate to the study's subjects of interest, for example, different types of environmental strategies, external and internal drivers of strategy change, and MCS design, use and objectives. Each instance was coded as a node in Nvivo8. This coding is based on the identification of key words or phrases and the reading and interpretation of the underlying meanings of each sentence or paragraph of the interview transcripts and documents. During the coding process, additional nodes for the patterns suggested from the reflection process were established (Section 5.4). Simultaneously with being coded to the relevant nodes, data were also classified into different generators and time periods to facilitate longitudinal analysis of the individual cases, as well as inter-generator examination.

\subsubsection{Data analysis}

Once the data were coded and classified, they were analysed using qualitative and quantitative techniques. Qualitative content presents itself as quotations or sentences that were coded in NVivo nodes. The qualitative analysis focuses on interpreting the 
text (i.e. the coded content) to discern the meanings and thinking that underlie the opinions and statements given by particular individuals or organisations, and through which, theoretical explanations can be achieved (May, 2001; Silverman, 2006). Accordingly, qualitative analysis was undertaken in three steps. Firstly, for each generator, coded data were analysed to understand the patterns of, and relationship between, GCCP-related drivers and strategic and MCS changes that occured within each period. Secondly, results were then compared across the five periods to identify potential dynamics and change in such patterns/relationships over time. Thirdly, the patterns and relationships found in one generator (Firm A) were compared to those in other generators (B, C, D and E) to gain insights into inter-generator variations and potential drivers of such variations.

In contrast, the quantitative analysis is based on indicators, which were acquired in two ways. Firstly, some indicators were readily available through the interviews and documents, such as generators' emission profiles, generation volume, retail bases, total assets, employee numbers, and the number of carbon credits owned. These factors were used to assess the GCCP-related exposure of each generator (and how such exposure changed over time) and the availability of organisational resources. Secondly, the factors identified from the qualitative interpretation of interview data and supported by documentary evidence were assigned quantitative values (i.e. measurement); for example, impact of external and internal drivers on each generator, level of strategy proactiveness, and degree of MCS design and use. These values were also coded to relevant nodes in Nvivo8. Quantitative measurement and analysis are discussed in more detail in Sections 5.7.2.2 to 5.7.2.4. Further, using matrix data displays, quantitative analysis is combined with qualitative analysis, as discussed next.

\subsubsection{Matrix data displays}

The combination between quantitative and qualitative analyses has the potential to enhance analysis of the issues/factors being studied and yield richer insights (Lillis and Mundy, 2005). This combination is achieved by using matrix data displays, in which data are displayed in terms of both indicators (quantitative) and quotes (qualitative) (Eisenhardt, 1989; Miles and Huberman, 1994). There are various schemes of data classification in data matrix displays, including classifying by case or organisation studied (case-ordered), by time period (time-ordered), or by pattern or theme (themeordered). To enable both longitudinal and between-firm comparative insights, this study employs all the three schemes of classification: case-ordered, time-ordered, and theme- 
ordered. Tables 5-2 to 5-6 provide extracts from the data matrix displays to be used for data analysis.

Table 5-2: Matrix data display for GCCP-related external drivers

\begin{tabular}{|c|c|c|c|c|c|c|c|c|c|c|c|}
\hline \multirow[b]{3}{*}{ Firm } & \multirow{3}{*}{$\begin{array}{l}\text { Time } \\
\text { period }\end{array}$} & \multicolumn{10}{|c|}{ Major GCCP-related external volatilities or pressures } \\
\hline & & \multicolumn{2}{|c|}{ Jan00 - Mar02 } & \multicolumn{2}{|c|}{ Apr02 - Dec05 } & \multicolumn{2}{|c|}{ Jan06 - Sep07 } & \multicolumn{2}{|c|}{ Oct07 - Sep08 } & \multicolumn{2}{|c|}{ Oct08 - Oct09 } \\
\hline & & Indicator & Quote & Indicator & Quote & Indicator & Quote & Indicator & Quote & Indicator & Quote \\
\hline Firm A & $\begin{array}{l}\text { Policy and } \\
\text { regulatory } \\
\text { change }\end{array}$ & 1 & & 1 & & 2 & & 3 & & 3 & \\
\hline & $\begin{array}{l}\text { Changing fuel } \\
\text { economics }\end{array}$ & 1 & & 1 & & 2 & & 3 & & 3 & \\
\hline & Total impact & $\mathbf{X}$ & & & & & & & & & \\
\hline Firm B & $\begin{array}{l}\text { Policy and } \\
\text { regulatory } \\
\text { change }\end{array}$ & 1 & & 1 & & 1 & & 2 & & 2 & \\
\hline & $\begin{array}{l}\text { Changing fuel } \\
\text { economics }\end{array}$ & $\underline{0}$ & & 3 & & 3 & & 3 & & 3 & \\
\hline
\end{tabular}

Key: 0: not relevant/applicable; 1: low (operational) impact; 2: medium impact; 3: high (strategic) impact

Table 5-3: Matrix data display for GCCP-related internal characteristics

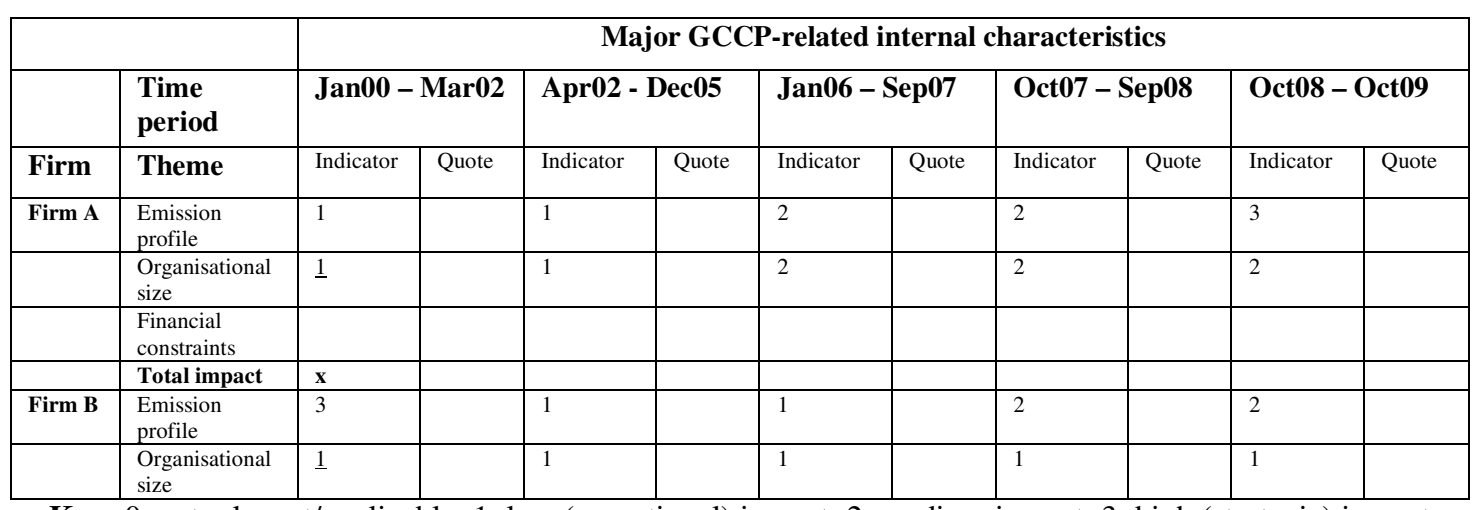

Key: 0: not relevant/applicable; 1: low (operational) impact; 2: medium impact; 3: high (strategic) impact

Table 5-4: Matrix data display for internal capabilities

\begin{tabular}{|l|l|l|l|l|l|l|l|l|l|l|l|l|}
\hline & & \multicolumn{7}{|c|}{ Major GCCP-related internal capabilities } \\
\hline & $\begin{array}{l}\text { Time } \\
\text { period }\end{array}$ & \multicolumn{2}{|c|}{ Jan00 - Mar02 } & \multicolumn{2}{|c|}{ Apr02 - Dec05 } & \multicolumn{2}{|l|}{ Jan06 - Sep07 } & \multicolumn{2}{|l|}{ Oct07 - Sep08 } & \multicolumn{2}{l|}{ Oct08 - Oct09 } \\
\hline Firm & Theme & Indicators & Quotes & Indicators & Quotes & Indicators & Quotes & Indicators & Quotes & Indicators & Quotes \\
\hline Firm A & $\begin{array}{l}\text { Technological } \\
\text { and financial } \\
\text { capabilities }\end{array}$ & 0 & & 1 & & 2 & & 3 & & 3 & \\
\hline & $\begin{array}{l}\text { Managerial } \\
\text { factors }\end{array}$ & 1 & & 1 & & 2 & & 3 & & 3 & \\
\hline & $\begin{array}{l}\text { Total } \\
\text { capabilities }\end{array}$ & $\mathbf{x}$ & & & & & & & & & \\
\hline Firm B & $\begin{array}{l}\text { Technological } \\
\text { and financial } \\
\text { capabilities }\end{array}$ & 1 & & 1 & & 1 & & 2 & & 2 & \\
\hline \\
$\begin{array}{l}\text { Managerial } \\
\text { factors }\end{array}$ & 1 & & 3 & & 3 & & 3 & & 3 & \\
\hline
\end{tabular}

Key: 0: not perceived/ not available, 1: limited capability; 2: some/moderate capability; 3: strong capability 
Table 5-5: Matrix data display for strategy proactiveness

\begin{tabular}{|l|l|l|l|l|l|l|l|l|l|l|l|l|}
\hline & & \multicolumn{7}{|c|}{ Proactiveness of environmental strategies } \\
\hline & Time period & \multicolumn{2}{|c|}{ Jan00 - Mar02 } & \multicolumn{2}{|l|}{ Apr02 - Dec05 } & \multicolumn{2}{|l|}{ Jan06 - Sep07 } & \multicolumn{2}{l|}{ Oct07 - Sep08 } & \multicolumn{2}{l|}{ Oct08 - Oct09 } \\
\hline Firm & Theme & Indicator & Quote & Indicator & Quote & Indicator & Quote & Indicator & Quote & Indicator & Quote \\
\hline $\begin{array}{l}\text { Firm } \\
\text { A }\end{array}$ & $\begin{array}{l}\text { Environmental } \\
\text { policy }\end{array}$ & 1 & & 1 & & 2 & & & & & \\
\hline & $\begin{array}{l}\text { Generation } \\
\text { strategy }\end{array}$ & $\underline{2}$ & & $\underline{2}$ & & $\underline{1}$ & & & & & \\
\hline $\begin{array}{l}\text { Total strategy } \\
\text { proactiveness } \\
\text { Environmental } \\
\text { policy }\end{array}$ & $\mathbf{x}$ & & $\mathbf{y}$ & & $\mathbf{z}$ & & & & & \\
\hline $\begin{array}{l}\text { Firm } \\
\text { B } \\
\text { strategy }\end{array}$ & & & & & & & & & & \\
\hline
\end{tabular}

Key: 0: no response; 1: reactive; 2 : moderately proactive; 3 : very proactive

Table 5-6: Matrix data display for MCS use

\begin{tabular}{|l|l|l|l|l|l|l|l|l|l|l|l|}
\hline & & \multicolumn{7}{|c|}{ Degree of use of controls and control systems } \\
\hline & Time period & Jan00 - Mar02 & \multicolumn{1}{|c|}{ Apr02 - Dec05 } & Jan06 - Sep07 & \multicolumn{2}{|c|}{ Oct07 - Sep08 } & \multicolumn{2}{|c|}{ Oct08 - Oct09 } \\
\hline $\begin{array}{l}\text { Firm } \\
\text { A }\end{array}$ & $\begin{array}{l}\text { Budgetary } \\
\text { controls }\end{array}$ & & & 1 & & 2 & & & & & \\
\hline & $\begin{array}{l}\text { Emissions-related } \\
\text { disclosure }\end{array}$ & 1 & & 2 & & 2 & & & & & \\
\hline & Total MCS use & $\mathbf{x}$ & & $\mathbf{y}$ & & $\mathrm{Z}$ & & & & & \\
\hline $\begin{array}{l}\text { Firm } \\
\text { B }\end{array}$ & $\begin{array}{l}\text { Budgetary } \\
\text { controls }\end{array}$ & & & & & & & & & & \\
\hline & $\begin{array}{l}\text { Emissions-related } \\
\text { disclosure }\end{array}$ & & & & & & & & & & \\
\hline
\end{tabular}

Key: 1: some operational use; 2: used regularly at operational levels with some attention at senior levels; 3: strategic use by top management

The quantitative indicators and qualitative content coded in Nvivo8 will be exported to Excel to enable data display. The use of matrix data displays facilitates the presentation and examination of the factors and relationships between the factors in the cases and across time periods, and thus promotes analysis 'completeness' (Miles and Huberman, 1994). The matrix data displays also help determine the total impact of external and internal drivers on each generator and identifies the degree of change in environmental strategies and organisational MCS design and use, across all the five periods. This in turn allows the research question and the four propositions (P1, P2, P3, P4; Figure 4-2) to be assessed. The measurement associated with each of the factors presented in the matrices (Tables 5-2 to 5-6), is explained next.

\subsubsection{Measurement of GCCP-related external and internal drivers}

Prior studies (reviewed in Sections 3.2 and 3.3) use a variety of scales to evaluate the impacts of external and internal factors on organisations or environmental strategy formulation and implementation, such as, a 5-point scale (James et al., 1999; Eiadat et al., 2008) or a 7-point scale (Henriques and Sadorsky, 1996). Since it is the researcher rather than the respondent who quantifies the factors in this study, it is more appropriate 
to adopt a simpler ranking scale. Applying a similar ranking system to the one recommended by AS/NZS 4360:2004 for risk factor impact (Standards Australia and Standards New Zealand, 2004), a scale from 0 to 3 is used to evaluate the degree of perceived impact for each GCCP-related external volatility/pressure on each generator 0: not relevant/applicable, 1: low (operational) impact, 2: medium impact, 3: high (strategic) impact (P1) (Table 5-2). Using the same scale, internal characteristics are quantified based on the degree of their perceived impact on each generator during each period of GCCP change (Table 5-3). In contrast, for each time period, internal capabilities are assigned a value from 0 to 3, depending on their availability and strength as perceived by managers (P2): as 0 (not perceived or not available), 1 (limited capability), 2 (some/moderate capability), or 3 (strong capability) (Table 5-4). These quantitative values can also be summed to gain an assessment of the total impact of all the GCCP-related external volatilities and pressures, the total impact of all the internal characteristics, and the change in such impact over time (P1, P2). A basis is therefore provided to understand the extent generators change environmental strategies in response to these impacts $(\mathrm{P} 3)$. The summation also provides an indication of the total GCCP-related capabilities possessed by each generator during each time period and their implications for the generators' costs, competitiveness, and legitimacy, and hence their environmental strategies (P2, P3).

\subsubsection{Measurement of change in environmental strategies}

Change in environmental strategies is captured by two proxies: strategy proactiveness and strategic emphasis (Section 4.4.4). The literature in Chapter 3 highlights that environmental strategies are ranked along a continuum from no response, reactive, to proactive. However, there is a need to discern the change in the level of strategy proactiveness within a given strategy type. Hence, a proactive strategy can be further divided into moderately proactive and very proactive. The resultant scale for quantifying strategy proactiveness used in this study is therefore: 0 (no response); 1 (reactive), 2 (moderately proactive), and 3 (very proactive) (Table 5-5). The assigned quantitative values are also summed up across all environmental strategies to gain an aggregate value for the total strategy proactiveness of a generator within a time period. This summation facilitates a quantitative-based analysis of the potential association between a change in total strategy proactiveness and a change in total impact exerted by GCCP-related external and internal drivers, across generators and over time (P3).

While strategy proactiveness is quantified, strategic emphasis is assessed qualitatively to enable the identification of the primary environmental strategies that receive top 
management attention within a generator during a particular time period. This allows the potential linkages between change in strategic emphasis and the variations in the impacts of GCCP-related external and internal drivers, across different time periods, to be analysed qualitatively (P3).

\subsubsection{Measurement of change in organisational MCS}

From an MCS perspective, previous studies in environment-related MCS (reviewed in Chapter 3) are mostly qualitative and do not rank the design and use of MCS systematically. However, it is necessary to quantify MCS in this study so as to discern the total degree of change in organisational MCS across different GCCP periods (P4). Accordingly, the design and use of MCS are quantified in two steps. First, all the controls and control systems identified from interview data and documentary evidence are listed. Then, to represent MCS design within each generator, each control and control system is classified as i) available, i.e. already established (value $=1$ ), or ii) not available or applicable (value $=0$ ).

MCS use is only evaluated for those controls and control systems that are available in the generators. Therefore, depending on the level and frequency of use, the use of a control or control system is quantified as 1 (used occasionally or on an ad hoc basis), 2 (used frequently at operational levels and has some attention at senior levels), or 3 (regular and strategic use by top management) (Table 5-6). Additionally, where applicable, the controls and control systems are classified into relevant MCS component types, following Simons' (1991) four categories. New classifications will be set up for any controls and control systems that do not fit these categories. The assigned values of use will then be summed up across the controls and control systems comprising a MCS component type in order to get the total degree of use of that MCS type within each generator during each time period. This allows both inter-firm and longitudinal comparison of change in the use of different MCS component types. Furthermore, it enables analysis of the potential impacts of strategy proactiveness and strategic emphasis on such change in MCS use over time (P4).

In contrast to MCS design and use, MCS objectives are analysed qualitatively as they represent separate categories rather than a continuum (Section 4.4.5). This enables a qualitative assessment of the impacts of a change in generators' environmental strategies on MCS objectives (P4). 


\subsection{Summary}

The study adopts a LMC approach to examine the changes in environmental strategies and associated MCS of generators from 2000 to 2009. The resultant sample includes five generator cases, representing $91 \%$ of the electricity generation sector (by market share), and 28 external stakeholders. Interviews were conducted with key people in these generators (13 interviewees) and external stakeholders (32 interviewees). More than 700 documents were collected from electronic and archival sources to supplement the interview data. Interview data and documents are coded in Nvivo8 and analysed using qualitative and quantitative techniques and Excel-based matrix data displays. Reflection and triangulation strategies are used throughout the processes of sample selection, data collection and analysis to validate findings and enhance their richness and comprehensiveness.

Applying these different research approaches and techniques, a longitudinal and acrossfirm comparative analysis of changes in generators' environmental strategies and associated MCS as well as GCCP-related drivers of such changes, will be conducted. The next chapter, Chapter 6, presents the case-based evidence regarding the change in environmental strategies and MCS that each generator made in response to various GCCPs announced from 2000 to 2009. The subsequent chapters, Chapters 7 and 8 will present the results from the inter-firm comparative analysis of the evidence in order to understand the impacts of GCCP changes on generators' environmental strategies and organisational MCS. 


\section{Chapter 6 - Firm-based evidence of environmental strategies and organisational MCS (2000 - 2009)}

\subsection{Introduction}

In chapter 5 the methods for data collection and analysis were discussed. This chapter provides evidence of the longitudinal changes that occurred in each generator's environmental strategies and MCS in response to the GCCP changes in each of the five periods identified in Chapter 5. These are: Jan2000-Mar2002 (no GCCP); Apr2002Dec2005 (plan for carbon tax); Jan2006-Sep2007 (the ETS under development); Oct2007-Sep2008 (the ETS introduced and legislated); and Oct2008-Oct2009 (the ETS review). For each generator, evidence is presented relating to the GCCP implications for existing environmental strategies such as their generation investment, retail and political strategies, as well as new strategies formulated specifically in response to the GCCPs, such as emissions-related disclosure, internal and customer energy efficiency, and carbon credit strategies. Further, for each generator and within each time period, evidence relating to changes in the design and use of organisational MCSs to implement these environmental strategies are presented. Such strategy and MCS-related evidence will contribute to the answering of the research question and provide the validation for the four propositions:

P1: External GCCP-related economic and institutional drivers impact on generators' competitiveness, costs and legitimacy.

P2: Internal GCCP-related characteristics and capabilities impact on generators' competitiveness, costs and legitimacy.

P3: The impacts of GCCP-related external and internal drivers in turn lead to a change in environmental strategies.

P4: The change in environmental strategies requires corresponding modifications in organisational MCS.

Throughout this chapter, a reference to these propositions, where relevant, will be made, in short form as $\mathrm{P} 1, \mathrm{P} 2, \mathrm{P} 3$ and $\mathrm{P} 4$. 
The classification of each generator as either thermal or renewable generator depends on their historical generation asset base as at January 2002 (the start of the study period). Accordingly, generators with more than $60 \%$ of their generation volume coming from fossil fuels such as coal and gas are considered thermal generators, whereas renewable generators are those producing more than $80 \%$ of their electricity output from renewable sources, such as wind, water and geothermal. ${ }^{4}$ Following these criteria, Firms A and B are classified as thermal generators whereas Firms C, D and E are renewable generators. Further, the italics in this chapter represent quotes from either publicly available documents or interview data. The need to maintain anonymity for some evidence necessitates that in those cases, the source of the quote is not disclosed. The next section presents the evidence relating to Firm A - one of the two thermal generators.

\subsection{Firm A: a thermal generator}

Firm A was established as an independent state-owned-enterprise (SOE) in 1996, privatised in 1999 and is now a publicly listed firm on the New Zealand Stock Exchange. At its establishment in 1996, the generator had some of the oldest and least efficient thermal-based assets in the industry. In 2002, 63\% of its generation capacity came from thermal sources (gas). Since then, its strategy has been to invest in geothermal development and gas peaker ${ }^{5}$ projects. These efforts have resulted in a more balanced portfolio between thermal and renewable sources and reduced its level of carbon emissions, thus mitigating Firm A's exposure to the GCCPs. During the 19992003 period, the generator acquired a number of retail businesses and became one of the largest integrated electricity generators in New Zealand, with a 30\% and 28\% market share in the wholesale and retail markets respectively. Firm A's generation plants are located on the North Island, but its retail business covers both North Island and South Island. Evidence relating to the environmental strategies and the MCS implications in each of the five time periods from January 2000 to October 2009 is presented below.

\subsubsection{January 2000 to March 2002}

Prior to 2002, the generator's environmental policy was characterised by a primary focus on compliance with the requirements of the Resource Management Act (RMA) (P3). The installation of an environmental management system (EMS) for generation

\footnotetext{
${ }^{4}$ It is noted that geothermal energy is a renewable source (and considered to be so in this study), but it also discharges carbon emissions, though to a smaller degree than fossil fuels.

${ }^{5}$ A gas peaker is a gas-fired generation plant that runs only when there is a high electricity demand.
} 
plants contributed to the minimisation of non-compliance incidents (P4). Simultaneously, Firm A's management claimed that these efforts amounted to environmental improvement above and beyond specific RMA requirements. The generator did not have a separate environmental report, except for the disclosure of its environmental policy. In its annual reports (ARs), total emissions from generation activities were reported and qualitative descriptions of other environmental impacts from the thermal and renewable plants were provided (P4).

\subsubsection{April 2002 to December 2005}

When the Government signalled the intention to ratify the Kyoto Protocol in 2002, the generator recognised the importance of monitoring and mitigating the level of its generation-related carbon emissions in order to manage its social legitimacy (P2). In one of the ARs, Firm A highlighted that it:

[...] is committed to ongoing consideration of options to reduce or avoid the emissions of greenhouse gases from our generating plants, to help meet Protocol obligations.

Additionally, it engaged in discussions with policy makers and politicians regarding the design and implementation of GCCPs (P3). The generator also entered into the NGA, a low cost government-directed initiative to improve energy efficiency around different generation sites (P1, P3). However, in contrast to other generators, it did not enter the PRE scheme to receive carbon credits to support its renewable investments. The focus of Firm A's generation investment strategy remained with fossil fuels, but it began to investigate the economic viability of wind energy (P3). Consistent with this generation strategy, the generator argued in its political activity that thermal fuels remained a key part of New Zealand's future energy mix. In 2004, Firm A lobbied against a carbon tax on the grounds that the uncertainties and costs of guessing the wrong charge would lead energy firms to delay generation investments (P1, P3).

Due to the uncertainty surrounding New Zealand's Kyoto obligations and the nature of the proposed carbon tax, the generator did not consider the implications of a future carbon price or its associated costs in its investment decisions (P1, P3). Instead, the focus of its climate change actions was to "encourage our employees to seek new opportunities to improve energy use and assist customers with demand-side management”.” (Annual Report 2003). From 2003, the generator implemented, on a trial basis, a number of energy efficiency initiatives which included energy audits and 
offering energy efficient products to its customers. Further, in 2004, an energy efficiency strategy was adopted with significant enhancement to the energy efficiencyrelated advisory services provided to business customers (P3). Interviews with managers revealed this strategy was driven by pressures associated with initiatives from a government agency, the Energy Efficiency and Conservation Authority (EECA), rather than short-term internal efficiency needs (P1).

An environmental policy was adopted in 2002 which highlighted the generator's focus on ensuring compliance with the RMA resource consent requirements (P4). From 2003, the environmental objectives and targets set were adopted as an additional measure to improve environmental compliance (P4). It also streamlined its environmental management function, improved its EMS and achieved certification under ISO 14000 (P4). The emissions monitoring system was also updated in order to ensure compliance with the complex conditions of the generator's resource consents (P4). Additionally, in an attempt to show its efforts in reducing emissions, the generator began to monitor and measure the total generation emissions intensity and disclose it externally (P4). Environmental performance was also disclosed in separate Annual Environmental Reports rather than the ARs (P4). However, the environmental policy was reviewed in 2005, resulting in no environmental report being produced between 2005 and 2006 (P4).

\subsubsection{January 2006 to September 2007}

In June 2006, the generator joined the Sustainable Business Network. The SBN seeks to promote environmental quality, social equity, economic prosperity and ethics in business. This participation signalled that the generator was responsible and concerned about the social and environmental impacts of its operations. Simultaneously, SBN membership placed more pressures on the generator to revise the way it conducted business and reported its performance (P1). As a result, from 2007, the generator produced its first Sustainability Report in accordance with the guidelines of the Global Reporting Initiative $(\mathrm{GRI})^{6}(\mathrm{P} 4)$. This Sustainability Report followed a triple bottom line reporting format, which incorporates three aspects of the generator's performance: economic, social and environmental.

After the Government cancelled the carbon tax, a shift in policy was signalled relating to the development of an ETS (P1). This resulted in the generator adopting more

\footnotetext{
${ }^{6}$ GRI reporting is a network-based organisation that develops and disseminates a reporting framework and guidelines based on which organisations can report their sustainability performance (www.globalreporting.org)
} 
definitive measures to respond to climate change and reduce its emissions. A Climate Change Action Plan (the Plan) was announced, including not only efforts to promote internal and customer energy efficiency but also changes to its generation strategy, an active political strategy and increased disclosure of emissions (P3). Customer energy efficiency and internal energy efficiency (as discussed in Section 6.2.2) took on renewed importance in this Plan. In particular, the generator stated a serious intention to pursue improvements in internal energy efficiency by establishing a separate team and making a senior manager responsible for implementing energy efficiency plans (P4). To ensure effective implementation of the Plan, formal reporting systems and crossfunctional coordination systems relating to internal energy efficiency were intensively used at operational and strategic levels (P4). The generator also identified "climate change risks and opportunities" (interview with a senior manager) and integrated them into the company-wide risk register (P4).

The most significant difference in the generator's climate change action plan, when compared to its previous environmental policy (prior to 2006), was the change in generation strategy (P3). The generator publicly announced its launch of a renewable investment programme, with a billion-dollar capital commitment in February 2007. This announcement marked a strategic move from thermal-based generation to the pursuit of renewables (primarily geothermal) for new generation. This was stated as its primary strategy to reduce emissions from electricity generation. However, interviews with senior managers suggest this strategy shift was facilitated by a change in CEO in 2006, with the new CEO strongly supporting geothermal development (P2). Another driver was the anticipation of changing fuel economics as a result of the planned GCCPs (P1):

If you don't go down that direction the government may come and regulate you. Then some of our plants will be uneconomic because government will come along and say "you knew about this problem and you did nothing about this, you built a carbon emitting plant. It's too bad that you're going to make a loss on them (because of the new policy)”. (A Senior Manager)

The managers interviewed also revealed a number of internal capabilities that enabled the generator to implement its environmental strategies effectively (P2). These capabilities included "relationship and cooperation with the majority shareholder", "corporate culture" and "pressures related to the capital markets"." (interview with the wholesale manager). The majority shareholder had "a lot of expertise, good thinking and good management capability" (interview with the a senior manager) which has been 
shared and transferred to the generator through various cooperation projects (P2). The generator also had a corporate culture that was strongly focused on cost control ("every dollar matters") and high performance ("everybody asks whether it adds value to the firm") (P2).interviews with several senior managers) (P2). Having a private ownership structure led to a potentially different approach to generation investment in comparison to the other generators. The stock market continuous disclosure requirements resulted in "a great deal of focus on the company" but were "a tremendous way of discipline" on the generator's approach to generation investment (P2).interview with a senior manager) (P2). As commented by a senior manager:

The tendency in SOEs will be to undertake investments all the way down to investments that have a value that is close to the cost of capital. But we will only do investments that we believe have a margin on the cost of capital.

To implement its new renewable-based generation strategy, the generator modified its external fuel monitoring system to focus on exploring resource sites for geothermal development, as opposed to the concern with gas supply in previous periods (P4). Strategy meetings were held and internal newsletters distributed to build awareness of the new strategy throughout the organisation. The generator also pursued an active political strategy to support its generation strategy (P3). Policy changes were monitored intensively and discussed at top management levels to formulate appropriate strategic and political responses (P4). Accordingly, the generator strongly supported an ETS and the government's making of renewable investments a priority in the NZEI. In its submission to the draft NZES issued in December 2006, the generator challenged the whole industry to an ambitious emissions reduction target and strongly supported the 90\% renewable target in the NZES. It recommended that the Government consider geothermal as a strategic energy option instead of wind due to the wind's unreliability. Accounting-based analysis was employed in its submission to provide support to these recommendations $(\mathrm{P} 4)$.

Further, the generator also indicated its intention to "take a lead" in seeking "green" business opportunities (Annual Report 2006) (P1). Accordingly, it launched a number of retail campaigns to promote the use of energy savings products and won a number of awards for these campaigns (P3). These initiatives enhanced the generator's reputation and competitiveness:

It's all small stuff but for those customers it is great. They are advocate customers who go out there and say our firm is the nicest thing that happens to 
them, like, they did this for my business and saved me this much money... They are ultimately the customers that you want because there's nothing more convincing in selling a product than a customer going "Ah, these guys are brilliant!”. (A Senior Manager)

Despite the competitive benefits, Firm A's managersit was insisted that these strategies were driven by 'a genuine concern about climate change and a perceived need to do something about it'. (interviews with two senior managers). Additionally, they contended that the generator needs to behave ethically by responding to climate change challenges, so as maintain credibility with staff and customers (P1).

Compared to the previous periods, emissions management to prepare for future ETSrelated compliance was accorded a primary focus in the organisational MCS (P4). Compliance with RMA resource consents took on a more subordinate focus. This is partly because improvements undertaken in the last period, to maintain the ISO 14001 accreditation, had enabled the generator to achieve a high level of environmental compliance. Furthermore, updates in organisational MCS, such as the emissions monitoring system, were needed to minimise the potential inaccuracies in emissions monitoring and measurements and to reduce emissions levels (P4). While generation emissions had always been monitored to satisfy RMA requirements, the economic implications of the ETS associated with Firm A's generation emission profile meant that emissions monitoring needed to be more accurate (P1, P2):

Under the RMA, these things were not resolved and did not result in money going out of the door. So you just have to get accurate enough to satisfy local bodies. Whereas with money going out of the door [under an ETS], tens or hundreds of millions of dollars going, that requires a totally different kind of focus. (A Senior Manager)

\subsubsection{October 2007 to September 2008}

In 2008, while the ETS was being considered in the Parliamentary legislative process, the generator announced a new environmental policy with an explicit focus on mitigating environmental risks, including those associated with generation emissions (P2, P4). One manager interviewed suggested that this new policy reflected the increased importance being placed on emissions management, as Firm A recognised its potential ETS-related carbon costs (P3). The sustainability reports continued to include measures of total emissions and emissions intensity, along with the disclosure of other 
environmental performance measures (P4). The generator's non-generation related activities were certified as carbon-neutral for the period 1 July 2007 to 30 June 2008, and thus, total emissions from non-generation activities were not disclosed (P3, P4). Carbon neutrality certification, titled CarboNZero, is awarded by the LandCare Research Institute to organisations that take measures to reduce their carbon emissions and buy carbon credits to offset remaining emissions. The move to carbon neutrality was perceived by external interviewees as a marketing tool in the face of increasing consumer environmental concern (P1):

If you just decide that you will become carbon neutral, what does that mean? No one knows who you are! But there is recognition of CarboNZero; it sees itself as a premium brand. For companies, that [gaining the CarboNZero certification] means they will be able to market themselves as green and carbon and hence can gain extra price and revenue. (An External Consultant)

Internally, managers held different opinions regarding the purpose of pursuing a carbon neutrality programme (P3). While a marketing and brand manager claimed that carbon neutrality would bring customer benefits, a wholesale manager considered it important to maintain its reputation to existing and prospective employees $(\mathrm{P} 1)$ :

Most customers either don't care, or don't care enough to pay extra for it. The ones that do care are probably buying from [renewables generators] anyway. What really matters to me is [carbon neutrality] helps get people on board. I had one experience where a prospective staff said "look, you are wasteful, I don't want to work for you". That was an eye-opener. I realised that if we don't change ourselves, we can't get the most talented people.

Consistent with its new environmental policy, the generator implemented the billion dollar renewable investment programme and publicly announced its cancellation of a thermal generation project due to problems in obtaining resource consents and the economic implications of the ETS (P3). Within its renewable investment programme, geothermal energy is the strategic priority, as the combination of access to resource site (e.g. resource consent), technological opportunities and available internal expertise made geothermal generation efficient $(\mathrm{P} 1, \mathrm{P} 2)$ :

We've got the expertise in geothermal area. There's new technology coming through in that area which really opens up that prospect. It is even cheaper than 
wind. We also have the process of securing resource access to some geothermal fields well underway. (A Wholesale Manager)

The ETS legislation strengthened the geothermal priority by providing the guarantee that geothermal investments would remain attractive despite future fluctuations in the supply of fossil fuels. This is because ETS-driven carbon costs will change the relative economics between alternative fuels and make renewable generation more efficient. The potential increase in wholesale electricity prices due to the ETS will thus bring a windfall gain to renewable assets (P1). A senior manager explained:

Probably what the ETS does is it removes a risk in a sense that if the gas market was to become fuller through new discoveries or reworking of existing fields, [geothermal development] is still worth a go. The ETS is not the driver but is the second layer which reinforces and makes it happen.

Interestingly, the generator also invested in gas peakers and gas storage facilities (P3). By building thermal back-ups, the generator could take advantage of price premiums caused by supply shortages when there are low lake levels or little wind (P1). Additionally, the generator had generation sites that are "close to the gas storage and close to water" and very suitable for developing thermal peakers (Annual Report 2007) (P2). A senior manager saw this as "a unique opportunity" that they could invest in to bring "a competitive advantage that would be extremely difficult for competitors to replicate". Accordingly, carbon-related information began to be incorporated into generation investment decisions through an assessment of the impact of the carbon charge on electricity prices, and thus resulting potential gains/losses from the generator's renewable and thermal peaking assets (P4).

Similar to the last period, the generator's political strategy focused upon supporting an ETS, the renewable target and early entry of the energy sector into the ETS (P3). It advocated an opt-in for gas purchasers to directly manage their emissions liabilities, but opposed the proposed thermal ban, arguing that such intervention distorts incentives to invest in peaking plants. Accounting was used in its governmental submissions to support the arguments presented (P4). Its retail strategy was also modified. In addition to maintaining the customer energy efficiency initiatives from the last period, the generator started to develop energy efficiency products and services for commercial purposes (P3). Thus, the system used for product planning was modified to incorporate and monitor information on customer demands and feedback on these products and services (P4). This new business was "quite a success" and helped improve the 
generator's reputation in the market place. Internally, a corporate-wide waste management strategy was adopted that focused on minimising total paper usage and resultant waste in all offices and generation plants (P3). Accordingly, measures of total waste and waste reduction were reported in the sustainability report (P4).

The level of disclosure relating to the generator's emissions levels and GCCP-related environmental strategies significantly increased (P4). The senior managers interviewed insisted this increased disclosure was not due to increased public concerns and pressures, but reflected a genuine belief in climate change and the need to respond proactively. However, this claim was received with criticisms by the external interviewees who suggested that such disclosure was to reduce customers' resistance when the generator increases its retail prices to recover the ETS carbon costs (P1).

Consumers are going to pay more for their electricity. Particularly appalling, the electricity price has increased $70 \%$ in the last 10 years. That's about to go up again, about another 5 to 10\% due to the ETS. So it's more and absolutely important that the generators communicate their climate change responsibility and ETS liabilities otherwise people will blame individual firms and their market share will suffer. (An Industry Expert) (emphasis by the interviewee)

\subsubsection{October 2008 to October 2009}

Due to the change of Government in October 2008, the Original ETS was put on hold and the Moderated ETS Bill was released for public consultation. Firm A made a submission that in general opposed the reduced carbon price and the $50 \%$ obligations, arguing these concessions delayed changes in economic investments and the adoption of low-emitting technologies (P1, P3). However, it recognised these concessions would give policy certainty and provide time for businesses to learn how to reduce their emissions liabilities. (Submission to Moderated ETS Bill, 2009). While geothermal remained the priority in its generation strategy, the uncertainty around the ETS reduced the rate of its generation development and put some projects on hold (P3):

We currently hold consents to construct a number of geothermal plants. The sequencing and timing of these projects are dependent on a number of factors, including the carbon policies. So we won't make the decisions on these projects until these policies are finalised. (A Senior Manager) 
Additionally, the generator decided to further diversify its fuel options and generation portfolio, by continuing the gas peaker and gas storage projects and starting to investigate potential hydro projects (Annual Report 2009) (P3). Management believed that hydro investment would be the preferred fuel option to meet medium to long term energy needs, especially in the context of increasing climate change concerns.

Interviews with senior managers revealed the internal organisational characteristics that may increase Firm A's ETS-related carbon costs. Accordingly, they were concerned about the lack of internal expertise in carbon trading (P2) and were also worried about not having access to good quality and reasonably-priced carbon credits to meet their ETS liabilities (P2). As a result, the generator's carbon credit strategy was to tap into the carbon trading book of its majority shareholder, which due to the size of its carbonrelated liabilities, would be able to undertake projects (e.g. forestry) that generate their own carbon credits (P2, P3). By doing this, the generator incurs a lower carbon cost in meeting its ETS obligations than by buying on the international market (P2). Firm A did not plan to buy carbon credits early because its managers believed carbon prices could fall in the future and that there were significant uncertainties around the ETS as a result of the change of Government (P1). Although not buying carbon credits, the generator was building internal carbon trading skills, based around valuing the risks attached to carbon contracts $(\mathrm{P} 3)$.

The main thing is that you need to make sure you have competitive tension in the market that you potentially purchase from. You don't need to be an expert in a carbon market and all the details that are going on in the market - they will come to you via those suppliers and the tips that they offer. All you really need is expertise in valuing risks of the contracts they offer. (AWholesale Manager)

Further, the impact of the ETS on retail competition was perceived to be small. Firm A believed there was only a small proportion of its customers who are 'deeply concerned about the environmental issues enough to switch suppliers' and the difference in branding between itself and renewable-based generators was 'not a significant risk' (two senior managers) (P3). However, the managers interviewed agreed that not all customers are aware of the wholesale market operations and therefore could be misled by green marketing campaigns run by renewable generators (P1).

ManagersInterview data revealed many MCS changes, some already implemented and others planned, within the generator to prepare for meeting its ETS obligations and the management of carbon-related compliance costs (P3). First, the level of volatility 
associated with the generation emissions was entered into the risk register for regular measuring and monitoring (P4). The generator planned to base carbon credit purchases on its assessment of the volatilities in its emissions levels (P4):

We will try to match our purchase book as much as possible to the volatilities that we're expecting. For a peaking plant we might have a stock of carbon credits sitting there, or just buy when and as we expect the plant to run. Whereas for the combined cycle for which we know we're going to run at a reasonably high level, we'll try to buy two or three years in advance. (A Wholesale Manager)

Firm A started to measure and account for the carbon trading risk and determine the exposure limits of its carbon trading transactions (Annual Report 2008) (P4). A separate budget for buying and selling carbon credits was established, based on which cost targets could be set and variances tracked (P4). One manager revealed that the actual measurement of emissions was still the responsibility of environmental managers. Further, a separation of duties was implemented where the responsibility for managing carbon trading risk fell on the trading and wholesale team (P4). However, the financial measurement and reporting of carbon trading risk was the CFO's responsibility. In order to better manage these risks, the generator planned to integrate measures of emissions and carbon costs into managers' performance evaluation, based on the principle of controllability (P4).

Additionally, interview data indicate that in order to enable coordination between different functions in monitoring, reporting and managing emissions and carbon trading risks, existing cross-functional information systems and links were extensively used (P4). Accordingly, the trading team started to have access to all other databases, including that of environmental managers (P4). The treasury and accounting staff were able to tap into the trading team's database to enable monitoring and reporting. The treasury and accounting function assigned a dedicated employee to be responsible for "measuring and reporting how much risk is in the carbon book and whether (the traders) are trading within boundaries"." (interview with the Wholesale Manager). From 2009, this employee shared office space with the trading people in the trading room. The interviewed managers indicated the purpose of this was to enable timely and accurate reporting of carbon-related risk, while simultaneously ensuring adequate independence and effective monitoring of trading activities (P4). 
Disclosure of energy efficiency initiatives and emissions was significantly improved, with the reporting of both generation and non-generation emissions (Sustainability Report 2009) (P4). Non-generation emissions were broken down by office and by scope, consistent with the LandCare research's measurement methodology. Furthermore, the CarboNZero certification was maintained for non-generation activities for the period from 1 July 2008 to 30 June 2009. However, this certification was discontinued for 2009/10 period (P4).

\subsubsection{Summary}

In summary, in response to the various GCCPs introduced, Firm A changed its environmental strategies and organisational MCS from 2000 to 2009. Table 6-1 summarises the amount of Firm A-specific evidence that supports the different propositions, as presented in sections 6.2.1 to 6.2.5. It is noted that this table reflects the degree that evidence supports a particular proposition through the count of pieces of evidence. Thus, this count does not necessarily capture the perceived impact exerted by GCCP-related drivers on environmental strategies and organisational MCS.

Table 6-1: Count of Firm A-related evidence availability

\begin{tabular}{|l|c|c|c|c|c|}
\hline & Jan00 - Mar02 & Apr02 - Dec05 & Jan06 - Sep07 & Oct07 - Sep08 & Oct08 - Oct09 \\
\hline $\begin{array}{l}\text { P1: } \\
\text { Economic drivers }\end{array}$ & 1 & 2 & 4 & 5 & 3 \\
\hline Institutional drivers & 1 & 2 & 2 & 3 & 0 \\
\hline $\begin{array}{l}\text { P2: } \\
\begin{array}{l}\text { Internal characteristics } \\
\text { and capabilities }\end{array}\end{array}$ & 1 & 1 & 5 & 2 & 3 \\
\hline $\begin{array}{l}\text { P3: Change in } \\
\text { environmental } \\
\text { strategies }\end{array}$ & 1 & 6 & 5 & 7 & 7 \\
\hline P4: Change in MCS & 2 & 7 & 7 & 8 & 11 \\
\hline
\end{tabular}

The table suggests that Firm A perceived multiple economic drivers associated with an ETS from January 2006 (P1). These drivers, including changing fuel economics, carbon market fluctuations and changing retail competition, had implications for Firm A's competitiveness and costs. Additionally, Firm A was exposed to a number of additional institutional drivers, such as government pressures for energy efficiency, and societal and customer pressures for environmental responsibility, during the middle periods (Apr2002 - Sep2008). During the ETS development period (Jan2006 - Sep2007), Firm A started to recognise that it had many internal characteristics and capabilities that could potentially provide a market advantage or facilitate effective carbon cost management under an ETS. These include geothermal experience and expertise, possession of suitable generation sites, and a close relationship with the majority shareholder (P2). In response to the GCCP-related drivers, from April 2002 to October 
2009, Firm A made many strategic changes (P3) and modified its organisational MCS (P4). Firm A changed its generation investment strategy, adopted a carbon credit strategy, and implemented internal energy efficiency and carbon neutrality strategies. To support the chosen environmental strategies, significant changes to organisational MCS were made, such as the establishment of an emissions monitoring system, and carbon trading systems, and a carbon neutrality certification process. From October 2008, as Firm A prepared for the upcoming ETS, it implemented the highest number of MCS changes (P4). These MCS changes ensured that Firm A could control its ETSrelated compliance carbon costs as well as maintain its competitiveness and social legitimacy.

\subsection{Firm B: A thermal generator}

Firm B was established as a SOE in 1999 and remains state-owned. At establishment, the generator's generation portfolio was driven by fossil fuels. In 2002, its thermal sources (gas and coal) accounted for $67 \%$ of its total generation capacity and hydro generation accounted for the other $33 \%$. The unreliability of gas supply and low rain levels in recent years, resulted in increased reliance on coal for its generation. Consequently, compared to the other generators, Firm B had a high carbon emissions profile. Since establishment, the generator has focused its strategy on growing its thermal generation assets. Recently, however, it has started to investigate potential renewable development projects. Firm B is vertically integrated with a substantial share in both the wholesale and retail markets (19\% and 25\% respectively in 2003). Both its generation plants and retail business are based in the North Island. Evidence related to the changes in environmental strategies and organisational MCS in Firm B in response to the GCCPs in each of the time periods from January 2000 to October 2009 is presented below.

\subsubsection{January 2000 to March 2002}

During this period, with no announced GCCP, the primary focus of Firm B's environmental policy was on compliance with the RMA and resource consent requirements $(\mathrm{P} 1, \mathrm{P} 3)$. To improve its environmental compliance, the generator installed a number of EMSs in its generation plants, including an emissions monitoring system (Annual Report 2000) (P4). Additionally, the generator undertook a number of cooperative initiatives with community groups so as to mitigate its environmental impacts and improve environmental conditions in the areas that its plants were located (P3). In a number of public documents, Firm B acknowledged the aim of these 
initiatives was to increase the local community's acceptance of its generation operations and enhance its ability to extend existing resource consents and apply for the new ones (Annual Reports 2001 and 2002) (P1, P3).

Firm B's generation strategy was focused on thermals and they commenced building of a major efficient gas-fired plant. Additionally, management did not recognise the influence of potential GCCPs on its choice of generation projects (P3). Therefore the impacts of carbon costs or carbon prices were not integrated into its investment planning decisions:

We asked what influence a carbon tax or carbon trading would have on the company's behaviour. The [generator's] chairman said it would not influence its behaviour, but agreed that it would result in electricity prices increasing. (a senior officer in a government authority (2001/2002 Financial Review by the Commerce Commission)

Environment-related MCS in this period was predominantly compliance-focused (P4). The generator's EMS design was broadly aligned with the ISO 14001 standards. As part of the EMS, the resource consent database was upgraded to ensure compliance with relevant environmental regulations and resource consents (Annual Report 2002) (P4). Environmental reporting was integrated as a section in the ARs, in which carbon emissions from the thermal plants were measured and reported to comply with RMA resource consent requirements $(\mathrm{P} 4)$.

\subsubsection{April 2002 to December 2005}

Once the Government announced the carbon tax in April 2002, Firm B recognised a need to reduce emissions from thermal plants in order to manage its potential carbon costs (P1, P2). A strategic diagnosis was undertaken to identify the "risks and opportunities” brought about by climate change. (Annual Report 2003). Consequently, “climate change risks and opportunities" were assessed and entered into the companywide risk management system (P4), as noted in an interview with a Senior Manager.

One key risk associated with the carbon tax was the significant carbon costs Firm B would incur due to its high emission profile, thereby threatening organisational profitability (P2). Therefore, in 2004, continuous emissions monitoring system units were installed in all the thermal plants to provide real-time measures for different types of emissions, including carbon emissions (Annual Report 2005) (P4). Additionally, 
senior management was briefed regularly on total carbon emissions from generation activities, with a focus on coal-fired plants (P4). However, these generation emissions were not disclosed externally (P4). A Director recalled:

We had total emissions reported monthly in the Board papers, for quite a while, to understand and monitor the implications of a carbon tax on the firm. However, we didn't talk about carbon emissions publicly and the emissions numbers were not disclosed in detail to the public. There was insufficient public demand for such reporting at that time.

Furthermore, Firm B recognised that traditional means of ensuring efficiency gains at generation plants were not sufficient to reduce GHG emissions and associated carbon costs and thus, started to look for more innovative approaches. Accordingly, carbon capture and storage (CCS) technology was investigated as a potential solution (Annual Report 2004) (P3). Further, while keeping the focus on thermal generation, the generator started to investigate potential renewable investment projects (P3). From 2003 to 2004 , the generator received more than 330,000 Kyoto carbon credits for its proposed renewable projects under the PRE scheme (MfE, 2009d) (P3). The granting of these carbon credits was a direct subsidy from the Government to enhance the economic viability of renewable projects (P1). Further, the generator participated in the local and national policy processes to argue for changes in RMA processes to facilitate new renewable investments (Annual Reports 2003 and 2004) (P3). These changes would enable Firm B to change its generation infrastructure to a stronger renewable base with lower carbon costs.

Energy efficiency was recognised as important in meeting future energy needs and reducing carbon emissions. From 2004, under EECA energy efficiency pressures, the generator provided advice to small business customers on conserving energy (P1, P3). As indicated by a senior manager, "we understand the responsibility we have in encouraging our customers, stakeholders and staff to use energy and resources wisely" (P1). Simultaneously, the economic downsides of such customer energy efficiency initiatives were recognised:

You can only lead customers so far, by giving them advice. If anybody does not want to turn lights off or insulate their hot water, not any amount of persuasion will make them do it. Furthermore, if we try to persuade people to use less power, obviously it means less revenue [for us]. (A Senior Manager) 
Internally, Firm B developed guidelines and trialled some measures to improve waste management and energy efficiency practices (P4). From 2004, a whole-of-company structure was established to allocate responsibilities for internal emissions reduction initiatives, and specific financial provision was assigned for each initiative (Annual Report 2004) (P4). Non-generation emission monitoring systems were established to measure and report emissions from non-generation activities (P4). Similar to the previous period, emission reporting was integrated into the AR. However, a difference was that the environmental section featured a discussion on climate change and highlighted the actions taken by the generator to reduce its generation and nongeneration emissions (P4).

Firm B was also exposed to increasing Government and societal pressures regarding its generation emission profile (P1). Therefore, in 2005, with approval from the board, management began to adopt a more proactive approach to climate change and GCCPs (Annual Report 2005 and interview with a director). An internal strategic planning process for responses to climate change was initiated, following a participative approach between the different levels of management. As commented by one Director (P4):

The senior managers were involved in the planning process. And they report to the Board on what's happening. It's a top down approach but also bottom-up in terms of the staff coming up with ideas for non-generation carbon savings. It is really another component that has been added to many people's jobs.

Consequently, a climate change plan was announced around mid-2005 (P3). This plan incorporated areas of generation emissions management, climate change policy oversight, customer energy efficiency, internal waste and energy efficiency, and emissions monitoring and reporting (Annual Report 2006). As part of this plan, previous initiatives in customer and internal energy efficiency were emphasised as part of Firm B's response to climate change. Generation emissions management was considered critical because of the generator's high emission profile and thus the associated carbon cost implications were of prime importance. Further, the generator believed substantial experience in emissions reduction developed in the past due to RMA carbon-related compliance would enable it to manage carbon costs effectively (P2). Further, emissions monitoring and reporting was assigned high importance, because it allowed management to monitor emissions-reduction initiatives and demonstrate the generator's responsibility in managing its emissions to the public (interviews with senior managers and directors) (P4). 
However, the most significant component in Firm B's environmental strategy was a shift in focus relating to generation investments (P3). Management increasingly recognised that continued reliance on coal-fired generation would result in significant emission levels and compliance costs under the carbon tax (interview with a director) (P2). Instead, to effectively reduce its generation emissions and carbon costs, Firm B needed to develop renewable-based plants to replace thermal ones. Consequently, the generator announced its intention to increase its renewables portfolio in almost all commercialisable sources, including wind, geothermal, hydro and tidal energy (Annual Report 2006). It also began to incorporate the expected value of the avoided carbon costs in assessments of potential renewable investment projects (P4). Simultaneously with increasing renewable investments, the generator started a thermal peaking plant project. Interview data and publicly available documents suggest this dual investment strategy created the operational and strategic flexibility required to respond to volatilities arising from GCCP changes (A media release by Firm B in 2005) (P1). Furthermore, this thermal investment was possible because the generator had a relatively secure fuel supply, due to its shareholding interests in a number of gas exploration projects (interview with a Senior Manager) (P2).

\subsubsection{January 2006 to September 2007}

Once the carbon tax was cancelled in December 2005 and the Government began to develop an ETS, senior management was updated regularly on its generation emissions level and carbon prices on international markets (interview with a director) (P4). Due to this information, Firm B recognised the substantial impacts that an ETS would have on the costs and profits of existing generation, as well as the viability of its thermal projects (P1). In response to the draft NZES (issued in December 2006), it argued against the proposed thermal ban and considered the $90 \%$ renewable target "unaffordable" (P3). Using accounting-based methods, Firm B quantified the impacts of the proposed NZES emissions reduction model and argued this model would lead to high price increases and threaten SoS (P4). Thus, the NZES would result in "extremely high costs to the electricity system and consumers" (Submission to the draft NZES, February 2007). Additionally, the generator emphasised the importance of fossil fuels in NZ energy future and argued for more recognition of CCS technology and its role in future emissions reduction. This would protect Firm B's current thermal assets and CCS investments in the face of Government preference for renewable energy (P1). 
In this period, Firm B began to implement the climate change plan announced in mid2005. While continuing previous initiatives relating to customer and stakeholder energy efficiency, it also sought to differentiate itself from other generators (P3). Instead of developing emissions calculators, Firm B chose to focus on educational tools such as an on-line game that taught children about sustainability. Furthermore, to achieve its internal energy efficiency objectives, a whole-of-company structure was set up; the CEO was named "the Climate Change Champion" and cross-functional teams were formed to implement emissions reduction initiatives (Annual Reports 2006 and 2007) (P3). Each initiative was given a financial provision but required no additional personnel and a company-wide emission calculator was used to keep track of nongeneration emissions (P4).

Measuring and reporting emissions was accorded a high level of importance in this period, as it formed one of the five key pillars of Firm B's climate change plan (P3). Therefore, Firm B implemented real-time monitoring of generation emissions and adopted an emission intensity measure as the key performance indicator used to monitor the performance of the emissions management strategy (Annual Report 2007) (P4). Targets were also set for generation and non-generation emissions reductions and a framework for emissions reporting was established (Annual Report 2007) (P4).

Despite the adoption of the emission intensity measure, the Board continued to place emphasis on total emissions levels (P4). Interviews with managers suggested the emission intensity indicator served to demonstrate the generator's environmental responsibility and thus was merely considered a response to increasing societal pressures (P1). Total emission levels remained “the key driver" of Firm B's future compliance costs and thus a priority from the senior management perspective (comment by a senior manager) (P2). In its external reporting, Firm B disclosed total emissions and emission intensity levels, from generation and non-generation activities (P4). Furthermore, in alignment with increasing governmental and societal focus on sustainability (P1), the generator prepared a corporate social responsibility (CSR) report in 2006 and a sustainability report in 2007 and included them in its ARs (P4). Its 2007 sustainability report was reviewed for completeness and materiality by an external consultancy company, demonstrating a further effort to gain legitimation (P4).

\subsubsection{October 2007 to September 2008}

As the ETS was passed into law, Firm B for the first time publicly acknowledged that "the impact of climate change is one of the foremost strategic challenges facing the 
company" (Annual Report 2008) (P1). An internal estimate of Firm B's ETS position (i.e. potential carbon costs) led the generator to review the 2005 climate change plan and divide it into a CSR Strategy and a Carbon Strategy (Annual Report 2008) (P3). The CSR Strategy dealt with the voluntary aspects of the climate change plan, including those initiatives previously under the internal and customer energy efficiency areas (P3). In contrast, the Carbon Strategy's aim was to manage the generator's carbon-related obligations and compliance costs in the short and long term (P3). In the long-term, the generator would reduce its emissions by increasing renewable investments and integrating the CCS technology into generation activities (P3). However, in the short term, its strategy was to buy carbon credits early to meet its ETS obligations. Interviews with managers suggest that the separate Carbon Strategy was a move to prioritise organisational resources into managing the carbon costs associated with the generator's prospective ETS obligations (P3). This was a necessary strategic choice because Firm B had the highest emissions level in the industry and needed to manage its emission levels and carbon costs effectively in order to remain profitable (P2).

To implement the Carbon Strategy, a budget for carbon trading was established (P4) and executives were sent overseas to buy "large lumps" of carbon credits "as they become available through forward contracts" (Annual Report 2008) (P3). This strategy was facilitated by the appointment of a carbon manager and the establishment of a carbon trading team, a carbon price monitoring system and carbon-related spending limits (P4). The team conducted intensive training for senior and middle management about how the ETS would operate and its economic impacts on organisational performance. A Director recalled:

We had some training... we spent a couple of days where we looked specifically at carbon trading, carbon unit pricing, and that sort of thing, which I understand, at that stage no other electricity firm's board has done. We have a number of carbon traders who understand the system and we don't need consultants to explain that to us.

Firm B's political efforts focused on making a submission to the ETS Bill. Its submission was the longest of all submissions, and was supported by extensive scenario analysis highlighting the impacts and consequences of the Original ETS on future electricity prices and generation investments (P4). In particular, it was contended that the Original ETS would result in a 'disproportionate loss' for Firm B compared to renewable generators (P1). Firm B argued strongly for an allocation of free carbon 
credits and a price cap to compensate for this loss. This indicated an attempt by the generator to externalise the emission reduction responsibility and carbon-related costs to the Government instead of managing them internally through technological and infrastructure change (P3).

Firm B reported its generation emissions in total and by intensity levels. Additionally, for the first time, in 2008, Firm B prepared its sustainability report using the GRI reporting guidelines $(\mathrm{P} 4)$. One interviewee noted an increase in managerial emphasis on emissions-related disclosure during this period (P4), and explained that such increase was driven by societal pressures (P1):

Why more reporting of bad numbers? I don't know that such disclosure brings any benefit. I think it is just a public expectation that we report our environmental performance, and in particular, our generation emissions. (A Director)

Firm B claimed to achieve carbon neutrality status for its non-generation activities in 2008, but this status was not externally verified (Annual Report 2008) (P4). An examination of archival data revealed little reduction in non-generation emissions through internal efficiency initiatives. Instead, carbon credits were purchased to cover most of these carbon emissions (P3). Further, cross-functional teams for internal energy efficiency were disbanded in 2008 due to a perception by top management that these teams had failed to motivate significant behavioural changes (Annual Report 2008) (P4).

\subsubsection{October 2008 to October 2009}

Due to the change of Government in September 2008, there was substantial uncertainty relating to the future of the ETS, coupled with strong Government pressures on the SOEs to deliver a higher rate of return (P1). In its submission, Firm B supported the changes in the Moderated ETS Bill, especially the proposed carbon price cap and the $50 \%$ obligation, since these ETS changes potentially halved its carbon costs (P1, P3). However, Firm B argued strongly that carbon costs may not be passed fully through to electricity prices and formally acknowledged that due to its high emission levels, it was "the company in the electricity sector most challenged by changes in the energy market place" (Submission to the Moderated ETS Bill, October 2009) (P1, P2). An estimate of its ETS carbon liability of a few hundred million NZ dollars per annum was disclosed in 
this submission (P4). A Director explained the "huge business challenge" faced by Firm B:

The main impact is on the balance sheet, because of the sheer dollar value of carbon credits that will have to be bought. You've got hundred million dollars in carbon costs and at the same time the minister of the SOEs said he wants a better return out of the power generators. So it is a challenge. If you suddenly have that huge chunk of costs, what do you do, would you put the prices up and lose customers to other power companies?

An interview with a Senior Manager revealed that emissions management was difficult, because of the growth in electricity demand $(\mathrm{P} 2, \mathrm{P} 3)$ :

One of the things we find with New Zealand is that, the demand volumes are becoming less and less seasonal by season, which put the pressures on all of our thermal plants. There is a little bit of opportunity to back off from coal and favour gas, but it's not quite as much choice as you might think. (A Senior Manager)

Further, the generator publicly admitted its concerns with "losing its acceptability to society" due to its thermal generation plants and associated emissions (P2). One Director expressed their frustration with the negative publicity the generator had received regarding its generation activities $(\mathrm{P} 1)$ :

We are only generating power because people are running appliances, businesses, and factories. But the newspapers are saying that the generator is a dreadful company because it's generating an awful lot of carbon emissions. Well, we are only doing that because people want to use power to do stuff.

Firm B decided to use the PRE carbon credits to offset its ETS surrendering obligations, with "the difference being sourced from domestic and international markets" (Annual Report 2009) (P2, P3). This strategy would enable the generator to manage its ETS compliance costs as well as reduce its exposure to carbon market fluctuations. Furthermore, in anticipation of the loss under the ETS, the generator wrote off a few hundred million dollars from its thermal plants' asset value, which led to an overall loss for the 2009 year (Annual Report 2009) (P4). However, a past senior manager revealed that it was a "smart strategic move" to mitigate the costs associated with future asset transformation from thermal to renewable-based. Additionally, this "Big Bath" helps 
satisfy the Government's return requirements and protects managers' bonus interests in the future:

With this write-off, the firm effectively depreciated almost all the historical cost of its thermal plants. The running of these plants in the future will deliver net revenue since there will be no depreciation expenses and wholesale prices only have to cover short run marginal costs such as the carbon charge and the cost of fossil fuels. (a retired Director)

Additionally, to further avoid carbon costs, Firm B decided to put some of its thermal plants into early retirement, arguing that "it is not our responsibility to provide SoS without adequate commercial compensation” (Annual Report 2009) (P3). Accordingly, its organisational mission statement was changed to emphasise the importance of making an adequate return for the shareholders (P4). Its generation investment strategy was further revised; renewables became the new focus and the renewable development programme was accelerated (P3). Management emphasised that "where it makes economic sense, renewables have to be the preferred option for new generation". This strategy change was motivated not only by the need to significantly reduce the generator's emissions profile and thus ETS liabilities, but also by a prospect of "gaining greater social acceptance as an operating entity" (Annual Report 2009) (P3). Consistent with this, estimated carbon costs became one of the most important considerations in all investment decisions (interviews with Senior Managers) (P4).

The delay in the ETS announced in October 2008 gave Firm B time for "learning-bydoing" and for re-evaluating its strategic position (Submission to the Moderated ETS, October 2009). To address the business challenges it was facing, a sustainable business model was adopted as an underlying principle to guide Firm B's business decisions (P3). Company-wide sustainability objectives, one of which was to respond to climate change, were established with an aim to move sustainability from being just an add-on to being integrated in core business (Annual Report 2009) (P4). These objectives were to be reviewed annually as part of the business strategy planning process. Internal waste and energy efficiency initiatives were also incorporated into the new sustainability objectives and maintained on an on-going basis (P4).

The new sustainability objectives led to a number of MCS changes. Energy audits were conducted for both direct and indirect energy use across all offices and operations. This led to energy consumption being measured, monitored and reported regularly to top management levels (interview with a senior manager) (P4). An environmental 
management framework was established to create a holistic approach to environmental management, including the monitoring and management of emissions (Annual Report 2009) (P4). For the first time, in 2009, Firm B had the sustainability content of its AR externally verified by one of the Big 4 accounting firms. Its managers believed that external verification would add further credibility to the generator's claimed commitment to sustainability (P4).

However, governmental pressures on SOEs to improve financial performance (P1) led to an internal restructuring (interviews with senior managers) (P3). A strategic review was undertaken for all of the generator's environmental strategies and as a result, financial performance became the predominant objective and assessment criteria for these strategies (P3). Therefore, internal cost control was tightened to improve operational efficiency, including a stronger use of budgetary controls (P4). As fewer resources were allocated to internal energy efficiency initiatives (P2, P3), related MCS such as non-generation emissions monitoring system received less strategic and operational emphasis (interview data) (P4). Simultaneously, despite the tight cost control, externally-oriented MCS such as emissions-related disclosure and AR verification were maintained since they were considered critical to mitigating the negative public perception associated with Firm B's emissions profile (P4).

\subsubsection{Summary}

In summary, the evidence presented above indicates that Firm $\mathrm{B}$ changed its environmental strategies and MCS significantly in accordance with its recognition of the impacts of the GCCPs on its business. 
Table 6-2 provides the count of evidence from 6.3.1 to 6.3.5 that supports the four propositions. 
Table 6-2: Count of Firm B-related evidence availability

\begin{tabular}{|c|c|c|c|c|c|}
\hline & Jan00 - Mar02 & Apr02 - Dec05 & Jan06 - Sep07 & Oct07 - Sep08 & Oct08-Oct09 \\
\hline P1: Economic drivers & 0 & 3 & 1 & 2 & 4 \\
\hline Institutional drivers & 2 & 3 & 3 & 1 & 3 \\
\hline $\begin{array}{l}\text { P2: Internal } \\
\text { characteristics and } \\
\text { capabilities }\end{array}$ & 0 & 4 & 1 & 2 & 3 \\
\hline $\begin{array}{l}\text { P3: Change in } \\
\text { environmental strategies }\end{array}$ & 3 & 7 & 4 & 6 & 7 \\
\hline P4: Change in MCS & 3 & 9 & 6 & 7 & 10 \\
\hline
\end{tabular}

The table suggests that Firm B recognised GCCP-related economic drivers most strongly in two periods: Apr2002-Dec2005 and Oct2008-Oct2009, when the preference for a GCCP (the carbon tax or the ETS) was clear. These drivers, including changing fuel economic and carbon market fluctuations, affected Firm B's competitiveness, and production and carbon costs (P1). It is also during these two periods that the implications of internal characteristics and capabilities were the most evident (P2). Accordingly, Firm B recognised that its high emission profile would lead to a significant amount of carbon costs. However, it also realised that its internal capabilities such as PRE carbon credits and experience in emissions reductions would enable the management of such costs (P2). Additionally, Firm B was exposed to many institutional pressures, such as Government pressures for energy efficiency and increasing societal pressures, primarily due to its high emission profile, during the middle periods (Apr2002-Sep2007) and the last period (Oct2008-Oct2009).

Table 6-2 also indicates that Firm B undertook many strategic changes during the periods with a defined GCCP presence (Apr2002-Dec2005 and Oct2007-Oct2009) (P3). In particular, it changed its generation investment strategy, adopted an active political strategy, and pursued emissions management and carbon credit strategies. Significant MCS modifications were implemented to support these strategic changes (P4). The highest number of MCS changes was observed from Oct2008 to Oct2009, as Firm B prepared for its compliance obligations under a prospective ETS. Hence, most of its MCS changes were to enable the building of internal capabilities for carbon cost management (P4). Additionally, some MCS changes were aimed at maintaining its competitiveness or responding to institutional pressures (P4). 


\subsection{Firm C: A renewable generator}

Firm $\mathrm{C}$ was one of the three state-owned generators established from the split of the ECNZ in 1999 and until now has remained state-owned. At establishment, the inherited generation assets were $100 \%$ renewable-based. Its generation investment strategy has remained renewable-focused since, moving from hydro projects in the 2000-2005 period to wind farms in later years. This focus led to the building of strong internal expertise in wind development. Additionally, a renewable-only generation portfolio results in no carbon costs under either a carbon tax or an ETS. Firm C is a vertically integrated generator, accounting for about $13 \%$ of the retail market and $29 \%$ of the wholesale market as at 2003. Its generation plants and retail business are based in both the North Island and South Island. The changes in Firm C's environmental strategies and MCS in response to GCCP changes from 2000 to 2009 are summarised below.

\subsubsection{January 2000 to March 2002}

Before any GCCP was introduced, Firm $\mathrm{C}$ was the first electricity firm to recognise the importance of climate change issues and New Zealand's potential obligations under the Kyoto Protocol in its annual reports. Its generation strategy was to optimise the operation of the hydro plants and wind farms inherited from the ECNZ's split-up (Annual Reports 2001 and 2002) (P3). Internally, it focused on staff training to increase staff awareness of environmental issues. In 2001, it joined the NZBCSD, an organisation set up by the Government to encourage sustainability practices (P1). This led to the adoption of a sustainability policy which emphasised a responsible use of natural resources and RMA compliance (P4). Consistent with its sustainability policy, the generator became the first in the NZEI to measure and reports its non-generation emissions (P4).

\subsubsection{April 2002 to December 2005}

This was a period of significant strategic reorientation in Firm C's generation investment strategy. Due to the announcement of the carbon tax in April 2002, it decided not to invest in coal (Annual Report 2003) (P3), because of the potential carbon tax implications of coal-fired generation (P1) and a lack of internal expertise (P2) (interview with a retired Director). During late 2002, it applied for resource consents for a major hydro project while simultaneously investigating possibilities for wind farms and peaking gas-fired plants to hedge the risk of hydro generation. In 2004, the hydro project was cancelled due to increasing unforeseen costs and the uncertainty associated 
with access rights to water. Consequently, Firm C's generation investment focus shifted to wind, because wind-based generation has low operating costs and no carbon emissions (P1, P3). Furthermore, wind was considered complementary to hydro and thus helped to manage the risk of a supply shortage in dry years (P3). A Project Manager recalled:

In 2004 when the [hydro] project was closed down, there was a reshuffle of our directorates and the Generation and Development directorate was set up to commercialise wind. By that stage, the core opportunity was with wind and the corporate and development arm felt that we are in for a much bigger proposal for wind.

In 2005, a strategic decision was made to exclusively focus upon renewables for generation investments (P3). Prior to this, senior management believed it was imprudent to focus only on renewables due to the unreliability of their supply. However, the Board took an active role in the planning process and convinced senior managers that investing $100 \%$ in renewable generation could create a green brand and enhance organisational market competitiveness and differentiation (P2). Furthermore, they foresaw the cost of carbon coming into future electricity prices which would make renewable investments economic (P1). A retired Director revealed:

We were always aware, in our planning as the Board, that the cost of carbon as a margin will be inevitable, through some mechanism, be it an ETS, or the carbon tax.

The decision to commit totally to renewable resources also reflected the internal awareness of climate change and "the need to do something about it", while simultaneously improving Firm C's social reputation in the face of increasing societal concerns regarding carbon emissions (interview with a retired Director) (P1). More importantly, it represented managerial recognition of the potential market advantage arising from a $100 \%$ renewable asset base (P2):

Being $100 \%$ renewable from the start was good positioning. Not everyone in the market can claim themselves as green, even though they could have invested in renewable generation quite a lot. (A Senior Manager)

As perceived by a senior manager, the combination of external and internal factors leading to this strategic decision is "a beautiful synergy" since it provided a distinctive 
opportunity for Firm C to gain a "green" competitive advantage (interview with a retired Director). Consequently, it started to pursue a green branding retail strategy (P3). Simultaneously, internal initiatives were implemented to encourage energy efficiency behaviour (P3). In response to the EECA pressures, Firm $\mathrm{C}$ developed tools and provided advice on energy efficiency for corporate and industrial customers and promoted sustainability practices among its partners and suppliers (P1, P3).

As part of its renewable-only generation investment strategy, Firm $\mathrm{C}$ initiated various wind development projects in New Zealand and Australia. In 2003 and 2004, under the PRE scheme, it was awarded over one million carbon credits for the potential reduction and avoidance of carbon emissions due to its proposed renewable projects (MfE, 2009d) (P3). These carbon credits bring additional income and enhance the viability of the proposed investments (P2). To further support this generation strategy, Firm C's political activities focused upon emphasising the importance of renewables for future energy needs and lobbying for improvements in RMA processes to facilitate renewable investments (Annual Reports 2003 and 2004) (P3).

Interview data suggest that the organisational MCS was modified to fit the renewablefocused environmental strategies. Top management used the HR system interactively to develop internal expertise in wind development (P4). A revision of the sustainability policy was undertaken to incorporate social and economic responsibility as opposed to the sole focus on environmental issues in the previous period (P4). Consequently, internal training and cross-functional communication systems were used extensively to promote staff awareness of sustainability issues and ensure the cooperation between different business units during the policy implementation (P4). The EMS was updated to enable the monitoring of both environmental compliance and environmental improvement initiatives (P4). Sustainability-related disclosure was initially incorporated as a separate section in the ARs. However, in 2005, sustainability became a guiding principle in preparing the AR (P4).

The scope of non-generation emissions monitoring and measurement was extended to include emissions from its subsidiary companies (P4). Thus, Firm C was the first generator to set emission reduction targets on a whole-of-company basis (P4). An interview with a middle manager revealed that these measures and targets were driven by climate change concerns and an internal need to control the increase in operational costs and emission levels due to rapid organisational growth (P2). Total non-generation 
emissions were also reported regularly to the Board and executive team, but not on a comparative basis with other generators (P4):

The emissions were in totals, but not on a comparative basis though. I would not be able to tell you how the non-generation assets were performing relative to compatible entities. That was possibly due to no other firm having published/disclosed the information at that time. We would be among the first to account for and measure that sort of thing. (a Director)

Although monitoring and reporting internal emissions, Firm $\mathrm{C}$ did not put an emphasis on the external disclosure of such data (P4). Its carbon footprint only had been published in the ARs since 2004. This was attributed to the lack of public and community demand at that time for emissions-related reporting (P1).

\subsubsection{January 2006 to September 2007}

With the cancellation of the Government's proposed carbon tax, Firm C acknowledged that the uncertainty relating to the GCCP presented "one of the major threats" to its business (P1). However, the international regulatory environment was becoming positive for renewable investments. To take advantage of this, Firm $\mathrm{C}$ decided to pursue a global energy generation strategy with a focus on wind development (P3). Large investments in renewable investments in the previous periods were seen as the enablers of this strategy $(\mathrm{P} 2)$ :

The scale of our investment in wind generation, and our continuing commitment to wind energy has given us a substantial market strength and allowed us to negotiate very favourable supply and equipment guarantees. (A Project Manager)

These investments and the renewable-only inheritance assets allowed the generator to develop a strong expertise in renewable development, putting it in "a powerful position in the market" (interview with a senior manager) (P2). Further, external technological advances had also reduced the unit cost of renewable generation, thus making it an economic choice compared to thermal projects (P1). Consistent with this generation strategy, Firm C lobbied the Government to price carbon through an ETS and provide further incentives for renewable investments (Submission to draft NZES, February 2007) (P3). 
From a MCS perspective, a Climate Change Framework for Action was developed (P4). Initially this was independent of the sustainability policy. A climate change team was established in parallel with this Framework and had "a short term mission to integrate into the business the capacity to identify and respond to risks and opportunities of climate change" (interview with climate change manager). However, one of the team's tasks was to incorporate climate change issues into the broader sustainability principles. Consequently, the sustainability policy from 2001 was replaced in 2006 with a new sustainable development policy that emphasised " $a$ commitment to being carbon neutral and to being a leader in climate change" (interview with climate change manager) (P4). The climate change team became the sustainability group and was in charge of implementing and monitoring energy efficiency initiatives and achieving carbon neutrality for the operations and activities across the organisation (P4).

Firm $\mathrm{C}$ became the first energy company in New Zealand to gain the CarboNZero certification (P3). This certification was consistent with Firm C's renewable-generation focus and reinforced its green brand. Managers considered certification to be " $a$ more tangible recognition than the renewable attributes" (interview with a senior manager). Thus, the CarboNZero certification was extensively advertised and integrated in the generator's retail strategy (P3). Additionally, in order to "transform customer experience" and tap into the increasing customer appetite for energy efficiency products (P1), a separate subsidiary was established to accumulate knowledge and develop products and services based on smart metering technology (Annual Report 2007) (P3). Energy efficiency advice was provided to residential customers and corporate/industrial customers. Firm $\mathrm{C}$ also extended the scope of its sustainability policy by developing sustainability guidelines for its partners and suppliers (P4).

This strategic and operational focus on sustainability was enabled by a strong concern for climate change amongst staff and an "entrepreneurial corporate culture" (interviews with managers) (P2):

There's genuine thinking about how to play our part in moving NZ to a low carbon future... People felt like they were part of something that was making a difference, which distinguishes us from other New Zealand corporates. (A Senior Manager)

That translates into their corporate culture being very committed to goals and to achieving success for the company. People who have worked for the company or have been involved with it have a lot of praise for it... It has this track record of 
doing stuff first, and well, and differently. Carbon neutrality is part of that ongoing innovation. (a retired Director)

Interview data suggest that the process of pursuing certification developed internal experience in emissions accounting and management in advance of an ETS (P4). Emissions were measured and reported to senior management quarterly (P4). Emissions intensity was adopted internally as a key performance indicator to manage and reduce emissions as part of CarboNZero certification process (P4). The generator disclosed the direct and indirect emissions for the core businesses (generation and retailing activities) following LandCare Research's methodology. In response to growing societal concerns (P1), the environmental section in its ARs featured a separate section highlighting the "risks" and "opportunities" associated with climate change and the GCCPs and the generator's actions to address them (interview with climate change manager) (P4). Additionally, the emissions disclosure often used words such as "sustainability", "commitment", "renewable energy", "emissions reductions", which were terms also used by the Labour Government in its policy documents and speeches (Annual Report 2007) (P4).

\subsubsection{October 2007 to September 2008}

In October 2007, subsequent to the announcement of an ETS by the Government, Firm $\mathrm{C}$ undertook a strategic assessment of climate change opportunities (interview with a senior manager). As a result, management recognised that climate change would bring about an opportunity to develop and deliver energy efficiency and carbon abatement products and solutions (P1). Further, an ETS was perceived to make renewable generation as efficient as, or even more efficient, than thermal generation (P1). The ETS also provided the context for Firm $\mathrm{C}$ to take advantage of its renewable-focused capabilities and increase its market competitiveness (P2):

The ETS is simply a symptom of what we have already tapped into which is generally greater awareness of environmental concerns and the ability to have, quite honestly, sold ourselves as the renewable generator and the ETS is just going to help us further in this area. The ETS provides us the context but we already have the core assets to enable that. (a Senior Manager)

To capture these opportunities, the generation investment strategy was extended beyond hydro and wind to investigating other potential technologies such as solar energy (Annual Report 2008) (P3). In its ARs, Firm C emphasised how its renewable 
generation strategy contributed to emissions reductions and thus was consistent with the goals of the planned ETS (P4). A green-brand marketing campaign was launched to reinforce its commitment to renewable energy (various media releases and television and online advertising materials) (P3). Additionally, Firm C started to sell efficient heating boilers in overseas markets, considering these boilers an innovative product that helped users to avoid or reduce carbon emissions while meeting increasing energy needs (Annual Report 2008) (P1, P3). In its political activities, to protect its renewable-based interests, Firm C strongly supported the ETS and urged the Government to provide further incentives for renewable investments so as to achieve a low-carbon energy mix in the future (Submission to the Original ETS Bill, February 2008) (P3).

As part of its vision to become a climate change leader, Firm $\mathrm{C}$ launched a trial online auction of carbon credits and participated in the development of New Zealand's first carbon credit trading platform (Annual Report 2008) (P3). Managers identified that the purpose of these initiatives was to gain experience in carbon trading and raise public awareness of climate change issues, which enhanced its organisational reputation and green competitiveness (interviews with managers). Additionally, Firm C successfully re-certified its electricity production and retail activities as carbon neutral under the CarboNZero programme (P3). Management saw carbon neutrality as reinforcing the credibility of its renewable energy commitment and as a way to retain and attract customers, especially with increasing retail competition (Disclosure of CarboNZero certification, 2007/2008) (P1). The Climate Change Manager summarised the benefits of carbon neutrality:

We integrate our retail strategies with CarboNZero branding. Every year when we think about renewing our certification, we ask ourselves whether our customers value this brand. Some of our customers do really value it. Many of our customers go for CarboNZero themselves and having a carbon neutral electricity company means that the emissions related to their purchased electricity are taken care of. We see the certification as a way to increase customer loyalty thing, say, trying to persuade customers from switching to other retailers. But we also want more people to switch to us. As more people become concerned to do something themselves for the environment they will potentially see switching to us as part of the solution.

A primary opportunity Firm C perceived with an ETS was the potential to enhance its profitability if electricity prices increased to reflect the cost of carbon (interview with 
managers) (P1). However, this windfall gain came at a cost. Public documents and interviews indicated there were "significant adverse public reactions" to its windfall gains, and the resulting pressures on it to return such gains to the consumers, without the recognition that Firm $\mathrm{C}$ had "responded to the environmental responsibility quite a bit earlier than anyone else" (climate change manager) (P1). Further, management was concerned that it would face intense competition from thermal generators in relation to carbon trading, thereby reducing its retail competitiveness (P1). As stated by the Climate Change Manager:

Our thermal competitors have an obligation under the ETS, and thus they have a stronger incentive to invest in carbon trading expertise. They are already quite active in this space. We see a threat of them developing that expertise and potentially taking away some of our customers due to their ability to offer advice and services related to carbon trading to help customers manage their ETS obligations and costs.

In realising its vision to become a climate change leader, a number of MCS changes were made ( $\mathrm{P} 4)$. The generator began to integrate climate change issues such as emission levels into the "business-as-usual" decision-making process (Annual Report 2008). To enable this, emission monitoring was conducted throughout the organisation and reported quarterly to the executive team and the Board (P4). Hard targets of emission reductions were set and soft initiatives were specified to support these target achievements (P4). In contrast to the previous period, emissions reduction targets were set in terms of emissions intensity rather than total emissions, as reducing total emission levels was considered not achievable due to organisational growth (interview data and Annual report 2008) (P2, P4). The generator had an overall budget for internal energy efficiency and CarboNZero certification programme, but not for specific emissionsreduction initiatives (P4). There were plans for setting up a "visual carbon budget" for each department/business unit so that unit managers could be held responsible for that budget and emission reduction targets (interview with the climate change manager) (P4). Emissions measures were also used extensively for staff training (P4):

We have a pretty good internal communications system on issues related to climate change and emissions. We have pages on our intranet. Our senior managers sometimes do talks around the business about this. We have online learning modules: Carbon 101 and Carbon 102. We do many training courses 
for the new employees, talking about why it's important and how we do it. (A Climate Change Manager)

Separation of duties and formal cross-functional reporting were important MCS aspects in implementing the internal energy efficiency initiatives and CarboNZero certification programme $(\mathrm{P} 4)$. Emissions data were collected by the procurement team and then submitted to the accounting team, where an accountant was in charge of developing the GHG inventories for the purpose of CarboNZero certification. These inventories were in turn submitted to the Climate Change Manager for sign-off who in turn submitted it to the executive team for review. The Climate Change Manager was also responsible for the certification budget and the sale and purchase of carbon credits.

There was "a single risk and opportunity document" for the whole organisation which identified the risks and opportunities of climate change. However, climate change risks and opportunities were monitored separately, through an opportunity register and a risk register for climate change (a Senior Manager) (P4). The latter fed into the companywide risk management system. As part of the CarboNZero certification process, emissions management plans were implemented by environmental managers and staff through the use of reduction targets and an emissions monitoring system. Information on actual non-generation emissions was transmitted to the financial management system where the accountants would use this information to calculate the organisation's carbonrelated exposure (interview data) (P4). In contrast, the opportunity register was part of a carbon-business development system that ensured opportunities were monitored and responded to by the business development team. Additionally, this team maintained a carbon market monitoring system to facilitate decision making related to carbon credit sales and purchases (interview data) (P4).

The method for measuring emissions was changed as part of the CarboNZero certification process. While previously this measurement only included air travel and the vehicle fleet, it was extended in this period to cover a wider scope of non-generation emissions (Annual Report 2008) (P4). Emissions measures started to be integrated into the company-wide balanced scorecard and linked to managerial compensation. Nevertheless, while using emissions intensity measures for its reduction targets, Firm C only disclosed total emissions in its ARs. It also started using GRI Sustainability Reporting guidelines to prepare the ARs but did not get the sustainability content of these ARs externally verified (P4). 


\subsubsection{October 2008 to October 2009}

From October 2008 to early 2009, before the Original ETS was suspended, Firm C continued the same environmental strategies as in the previous period. It acquired CarboNZero certification for the whole group (the organisation and its subsidiaries) for the third time in 2009 (P3). Further, the emissions of the whole electricity product lifecycle were accounted for, including shipping of wind turbines, construction of generation plants, purchased electricity on the wholesale market, and travel and fuel use by subcontractors (Disclosure of CarboNZero certification, 2008/2009) (P4). Total emissions and emissions intensity were measured and externally reported for the whole group (P4), and the emissions inventory was externally verified for the first time in 2009 (P4).

This comprehensive approach to accounting for emissions was seen by its managers as evidence of Firm C's commitment to and leadership in the climate change area which drove its ETS-related competitive advantage (interviews with managers) (P4). Additionally, the experience and learning gained from the ongoing certification process enabled the generator to become independent of external consultants:

For our first GHG inventory, [one Big 4 accounting firm] did it. The second time they helped us do it. The third time they advised us on a couple of logical issues. And this time round we did it all ourselves. (A Climate Change Manager)

From February 2009, in response to the ETS delay and proposed changes, Firm C undertook a comprehensive assessment of its organisational exposure to the ETS. It recognised that these ETS changes would dilute the potential ETS-driven cost efficiency of renewable generation and reduce the potential margins it could gain on the wholesale market (interview data) (P1). However, the proposed price cap was anticipated by its management:

Fortunately for us our carbon price forecasting has been pretty good, pretty accurate. We foresaw the cap and things. You'll recall the reaction earlier in the year to electricity price rises; it was absolute resistance even though that's what's necessary to enable the new renewable investments. So with the difference between where the carbon market might go and the political pressures to depress retail price rises, the cap seems inevitable. (A Senior Manager) 
Expecting such changes to the ETS, Firm C reduced its involvement in ETS policy processes and maintained a policy oversight function instead (interview data) (P3). It also reconfirmed its commitment to renewable-only generation investments in mid-2009 (Annual Report 2009). However, the uncertainty associated with the lack of agreement between the two major political parties (Labour and National) over the ETS design was recognised as a significant barrier in making long-term investment decisions (P1). Consequently, later in 2009, Firm C announced a delay in the issue of renewable notes to finance its planned renewable projects (P3). A senior manager revealed why they had postponed making decisions on some renewable projects:

Our decisions are made based on the policy provided by the Government of the day. But electricity generation investments are long-term assets, spanning 20 to 30 years. Not providing long term policy certainty results in substantial difficulty for us in making our investment decisions. (A Senior Manager)

From mid-2009, the Government put significant pressures on SOEs to increase their financial performance (P1). In his letter to all the SOEs' board chairpersons, the Minister of SOEs Simon Powell, stated:

The relatively poor (and declining) financial performance of SOEs, coupled with the challenging economic conditions, has brought us to the conclusion that change in the SOE portfolio is urgent and essential. [...] I think shareholders representing \$24 billion of taxpayers' money have an obligation to make sure entities are obtaining a decent financial return. (Trevett and Bradley, 2009)

In response, Firm $\mathrm{C}$ reviewed its strategic thinking and market positioning, the result of which was to focus on the core business of electricity generation and retailing and prioritise economic sustainability over environmental sustainability (P3). One Manager commented:

We are unwavering in our commitment to sustainability but looking at it in broader terms than just renewable generation and just emission management. It is about the fact that we need to ensure the sustainability of our business going forward. It is more about competitive positioning now.

Additionally, this strategic review led to a change in the sustainable development framework, with economic sustainability becoming the overriding objective for climate change initiatives $(\mathrm{P} 3)$ : 
One of the pillars of our strategy is to sustainably improve our earnings. It underlies the operational efficiencies that we get from reducing emissions and embracing energy efficiency. It's also the purpose why we want to attract customers who buy carbon zero certified electricity. (A Senior Manager)

From mid-2009, internal restructuring was initiated in an attempt to improve operational efficiency and thus lift the generator's financial performance (P3). The organisation's MCS was changed to focus upon internal cost control rather than on reducing emissions or implementing climate change initiatives (P4). Consequently, the role and importance of the climate change team changed:

Because we're supposed to focus on the core business and we don't have a compliance obligation under ETS at the moment, the management decided that we've got excess experts in carbon and climate change and we just don't need them. (A Climate Change Manager)

From September/October 2009, the financial resources allocated to climate change initiatives substantially reduced (P2). Consequently, the climate change team was disestablished, with existing team members being moved to different business units. Instead of having people specialised in carbon neutrality and carbon trading, these responsibilities became "just one attribute the person who does the mainstream function needs to have” (interview with the Climate Change Manager) (P3).

With these strategic and operational changes, a number of controls and control systems related to climate change initiatives were cancelled or used less (P4), including: the link of emissions measures into senior managers' compensation structure; the budget for carbon trading; and, emissions monitoring and reporting (interview data). Furthermore, the generator's 2009 AR focused on discussing economic sustainability, financial performance, productivity and survivability, which are the policy themes of the National Government (P4). This contrasted with the generator' predominant use of words such as "emissions reduction", "leader in climate change" in its AR disclosure in the previous periods under the Labour Government.

\subsubsection{Summary}

Evidence suggests that over the ten year period from 2000 to 2009, Firm C underwent significant changes in its environmental strategies and MCS to respond to the changes 
in the GCCPs. Table 6-3 provides the count of evidence for Firm $\mathrm{C}$ that support the four propositions as presented from 6.4.1 to 6.4.5.

Table 6-3: Count of Firm C-related evidence availability

\begin{tabular}{|l|c|c|c|c|c|}
\hline & Jan00 - Mar02 & Apr02 - Dec05 & Jan06 - Sep07 & Oct07 - Sep08 & Oct08 - Oct09 \\
\hline P1: Economic drivers & 0 & 4 & 2 & 6 & 2 \\
\hline \multicolumn{1}{|l}{ Institutional drivers } & 1 & 3 & 2 & 1 & 1 \\
\hline $\begin{array}{l}\text { P2: Internal } \\
\text { characteristics and } \\
\text { capabilities }\end{array}$ & 0 & 4 & 3 & 1 & 1 \\
\hline $\begin{array}{l}\text { P3: Change in } \\
\text { environmental } \\
\text { strategies }\end{array}$ & 1 & 7 & 5 & 6 & 6 \\
\hline P4: Change in MCS & 2 & 8 & 8 & 11 & 8 \\
\hline
\end{tabular}

The highest number of economic drivers (P1) was recognised during two periods of high GCCP certainty, Apr2002 - Dec2005 (the carbon tax) and Oct2007 - Sep2008 (the ETS). These drivers, including regulatory uncertainty, changing fuel economics, and carbon market fluctuations, affected Firm C's competitiveness. During the early period (Apr2002 - Dec2005), Firm C was exposed strongly to additional institutional pressures, such as government pressures for energy efficiency, and societal and employee pressures for environmental responsibility (P1). Further, Firm $\mathrm{C}$ recognised early on (during Apr2002-Sep2007) that its renewable-focused internal capabilities resulted in a significant GCCP-related market advantage (P2) Hence, since April 2002, Firm C has changed its environmental strategies significantly in the areas related to generation investment, carbon neutrality, internal energy efficiency and emissions-related disclosure (P3). These strategic changes were supported by a corresponding high number of MCS modifications from April 2002 (P4). Organisational MCS was changed most substantially from Oct2007 to Sep2008, when the ETS was announced and legislated and its significant economic implications were recognised (P4). The MCS modifications enhanced Firm C's green-focused competitiveness as well as improved its social legitimacy (P4).

\subsection{Firm D: a renewable generator}

Firm D is, and always has been a privately owned company. It became a publicly-listed company on the New Zealand Stock Exchange in the mid-1990s. Its traditional generation portfolio is $100 \%$ renewable-based with a predominance of hydro generation. From 2002 to 2009, a renewable-only generation investment strategy was pursued, focusing particularly on wind development and hence it has developed strong internal expertise in renewable technology. Firm D's business is vertically integrated between generation and retailing bases, accounting for $5 \%$ and $16 \%$ of the wholesale 
and retail market share in 2003. The generation plants and retailing business are located in both the North Island and South Island. In contrast to the other generators, Firm D is a net buyer, i.e. its retailing commitments exceed its generation capacity and thus it has high exposure to wholesale market fluctuations. Presented below is a summary of the changes in Firm D's environmental strategies and MCS associated with the changes in the GCCPs from 2002 to 2009. Due to a lack of data from publicly available sources and the interviews with managers, the period from 2000 to 2002 is not covered.

\subsubsection{April 2002 to December 2005}

Consistent with its renewable-based infrastructure, Firm D was a strong advocate of the carbon tax when it was announced in April 2002 (P3). It was acknowledged that the tax would have a positive impact on its decisions to proceed with renewable projects (P1). However, the generator considered the tax as only "the first step down the path to a market driven reduction of greenhouse gas emissions", and indicated its support of carbon pricing, i.e. an ETS, in the longer term (interview with a Senior Manager).

With a history of renewable-only generation and strong top management commitment to renewable development, Firm D started to investigate various renewable investment opportunities in New Zealand and Australia (P2; P3). They intended to proceed with these investments when they "meet or exceed its strict minimum rate of return criteria" (Annual Report 2004). The strategic focus was on wind development, since internal expertise in managing "wind project risks" provided a competitive advantage over other generators (P2). Based on the potential emission reductions from its proposed renewable projects, Firm D was granted over one million carbon credits by the Government under the PRE scheme (MfE, 2009d). Management revealed this grant had enhanced these projects' economic viability (P2). Additionally, some internal and customer energy efficiency initiatives were undertaken in response to the EECA pressures (Annual Reports 2002 to 2005) (P1, P3). However, these initiatives were limited in scope and received minimal top management attention (interview data).

As a net buyer on the wholesale market, Firm D was strongly exposed to wholesale price fluctuations (P1). Being a privately-owned generator also required in a higher rate of return on investment than state-owned ones (P2). Consequently, in 2003, Firm D exited some residential markets where there were an incumbent SOE retailer with a lower acceptable margin, to focus on "higher socio-economic market segments" (Annual Report 2004) (P3). This strategic shift also reduced the difference between its generation and retail bases and thus helped mitigate organisational wholesale exposure. 
Firm D recognised that its generation operations had the potential to significantly affect the environment and therefore a comprehensive EMS was developed with the ambition to "set a new industry standard in environmental excellence" (Annual Report 2004) (P4). A new environmental policy was developed in 2005 incorporating the concept of sustainability (P4). Accordingly, energy audits were conducted from early 2005, starting at head office and gradually rolling out to other offices and sites (P4).

Environmental measures were mostly qualitative in this period (annual reports and interview data) (P4). Due to its $100 \%$ renewable-based generation, Firm D was not required under its RMA resource consents to disclose carbon emissions. However, to enhance its reputation, the generator disclosed the emissions avoided with its proposed wind development projects. From 2002 to 2004, the generator did not produce a separate sustainability report. Instead it disclosed its environmental performance as part of the ARs. When the new environmental policy was released in 2005, a sustainability report was incorporated in the AR, but included the same sub-sections of social, environmental and economic performance as in previous years (P4).

\subsubsection{January 2006 to September 2007}

Significant GCCP changes were announced in this period, including the cancellation of the carbon tax plan and the termination of the PRE scheme. Firm D perceived these changes as a "double blow" to its renewable generation (Annual Report 2007) (P1). Further, no guidance from the Government on future GCCPs made it "difficult for many generation development proposals currently under consideration to be progressed" (Annual Report 2007) (P1). While Firm D had sufficient funding for these projects, it was concerned that the regulatory environment did not give a positive enough signal for them to proceed (interview with a senior manager). Additionally, the cost of imported wind turbines and hydro plants quickly escalated in this period mainly due to foreign exchange rate volatility, which altered fuel economics (P1). In response to these regulatory and economic factors, Firm D chose to be flexible in its strategic direction and continued to identify new potential investment projects and progress them to a point where "a commitment to construction can be quickly made when the current Government and regulatory uncertainties have been resolved" (Annual Report 2007) (P3). In Australia, where the regulatory environment was relatively more positive (P1), the generator continued its wind farm development and was granted renewable energy credits for its wind projects (P2, P3). 
Firm D began to publicly acknowledge the opportunities related to the GCCPs (Annual Report 2007) (P1). Management assessed that due to the $100 \%$ renewable focus in its generation development, it would be "in an excellent position to take advantage of the opportunities that a carbon constrained economy will bring" (interview with a senior manager) (P2). Firm D also developed carbon trading skills and expertise by entering into a number of contracts to sell its PRE carbon credits (Press release, November 2007) (P3, P2). Additionally, its renewable development projects were registered to gain other types of carbon credits (verified emissions reductions - VERs) that could be sold to voluntary markets (Annual Report 2007) (P2).

An active political strategy was also pursued to support its renewable investments. In its submission to the draft NZES, Firm D strongly advocated for NZES's focus on emissions reductions and urged the Government to develop policies to ensure that the cost of carbon was reflected in electricity prices (P3). Furthermore, the generator submitted to the Government a proposal that required thermal generators to surrender carbon credits to renewable generators to support the latter's new renewable projects (P3). Unsurprisingly, this proposal was strongly opposed by thermal generators (Submissions to draft NZES by thermal generators).

From a MCS perspective, a carbon market monitoring system was implemented to monitor carbon prices for carbon selling contracts $(\mathrm{P} 4)$. Due to the uncertainty about GCCP direction, the generator did not assess and integrate potential impacts of a GCCP into its investment planning (interview data). However, the potential changes in the GCCPs were monitored regularly and accounting-based analyses were used extensively by top management as they actively lobbied over the design of a future GCCP (interview data) (P4). Furthermore, the EMS was updated and appropriate personnel put in place to improve compliance with increasing resource consent requirements (Annual Report 2007) (P4).

Internally, the generator acknowledged that energy efficiency was increasingly becoming an integral part of an effective response to climate change (P3), rather than being merely a response to EECA pressures (P1). However, there was no company-wide monitoring system for non-generation emissions and no concrete targets to reduce these emissions (interview data) (P4). Further, its internal energy initiatives were limited in scope and only focused on electricity usage (P3). An energy efficiency guide was issued to encourage its customers to manage their electricity consumption (Annual Report 2007) (P4). Externally, there was cursory discussion of internal and customer energy 
efficiency as part of the sustainability report. However, the generator did not disclose the level of non-generation emissions nor the specific initiatives undertaken to mitigate such emissions (P4).

\subsubsection{October 2007 to September 2008}

Firm D strongly supported proposals for an ETS and the ten year moratorium on thermal generation (the thermal ban) when these GCCPs were announced in October 2007 (P3). Managers perceived that these GCCPs would bring about a positive regulatory environment for its renewable-only investment programme (P1, P2). To further its reputation, the generator stated publicly that its investment programme was designed "to help meet the sustainability targets set by Government, but, at the same time provide our present and future customers with a secure electricity supply" (Annual Report 2008). However, interviews with its managers and external industry experts revealed that the pursuit of these investment projects was driven primarily by the economic impacts of the renewable-favouring GCCPs (P1). Such GCCPs included the Government's financial assistance through the granted PRE carbon credits and the imposition of a carbon cost on fossil fuels which made renewables a competitive fuel choice for electricity generation $(\mathrm{P} 1)$.

With the introduction and subsequent enactment of the ETS, Firm D recognised the opportunity for "green branding", which aligned with its renewable-only generation assets (interview with a senior manager) (P1). It achieved CarboNZero certification for the first time in 2007. In 2008, this certification was extended to include some subsidiaries (P3). The motivation underlying the pursuit of the CarboNZero certification was that "certification provided the methodology and framework we felt was required to measure, understand and mitigate our emissions" (Disclosure of CarboNZero certification 2007/2008). More importantly, the generator considered that carbon neutrality was "a driver of shareholder value" by providing a competitive advantage in a carbon-constrained market (interview with a senior manager) (P3). Its management recognised that carbon-neutral electricity would attract corporate and industrial customers who also wanted to certify their businesses as carbon neutral (changing retail competition) (P1). Furthermore, the generator could sell the VERs received for its renewable projects to customers to help the latter offset emissions and achieve their climate change goals (Annual Report 2008) (P2).

To support the CarboNZero certification process, an emissions management plan was developed which established specific reduction targets in the five areas identified as the 
biggest contributors to the generator's total non-generation emissions (P4). These were fleet vehicle petrol and diesel, domestic air travel, international air travel, electricity, and waste to landfill (Disclosure of CarboNZero certification 2007/2008). Despite these efforts, there was a significant increase of $40 \%$ in emissions from domestic and international air travel, and electricity use, between 2007 and 2008 (Annual Report 2008). To achieve carbon neutrality certification, these emissions were offset using carbon credits generated from its own renewable projects (P2, P3). Additionally, a carbon trading system was established in the Trading and Risk Department to monitor carbon credit inventory and carbon market fluctuations (P4). The use of the carbontrading MCS helped develop carbon trading skills and provided information for decision-making relating to the sales of carbon credits (interview with a senior manager).

The sustainability policy was revised to reflect climate change issues and expand beyond environmental compliance to sustainability and economic objectives (Annual Report 2008) (P4). Consistent with the new sustainability policy, there was an increase in the disclosure of initiatives related to "sustainability" and "climate change action plan" in the ARs (P4). The generator claimed that the sustainability content in its ARs was prepared in accordance with GRI guidelines. However, a closer examination reveals this claim to be unsubstantiated since many measures reported were purely descriptive, and there were no quantitative measures for total non-generation emissions or waste, staff travel, and electricity use (P4).

\subsubsection{October 2008 to October 2009}

During this period there was a less positive environment for renewable investments due to the change of Government, the Original ETS review and the proposed carbon price cap (P1). Management perceived such environmental changes "influenced the timing of new investments and increased the difficulties and costs associated with bringing our investments to completion” (Annual Report 2009) (P3). A Senior Manager explained the impact of these ETS changes on the generator's investment strategy (P3):

We remain committed to $100 \%$ renewable generation investment. Therefore, we would only change our position when circumstances dictate that there is no other viable option. But we want to be flexible in relation to future regulatory changes. Due to the current policy and regulatory uncertainty, we have to delay making decisions on some of our renewable projects. 
Interviews with managers revealed that they expected changes to the ETS to occur. Consequently, they decided not to invest resources in ETS-related lobbying activities and instead shifted their focus to internal operations and activities in order to "enhance the long-term value of our company, and deliver improved returns to our stakeholders" (Annual Report 2009) (P3). To increase organisational profitability, the organisational MCS focused on internal cost control and revenue management in the wholesale and retail markets (P4). However, a policy oversight system was maintained to monitor and provide information on the legislative and regulatory changes to the ETS (interview with a senior manager) (P4). This information was reported to the executive team who would consider the implications of such information for the generator's environmental strategies (P4).

Additionally, Firm D perceived a change in retail competition due to "customers increasingly insisting that companies actually "walk the talk" in terms of claims about sustainability and eco-friendliness" (interview with a senior manager) (P1). Further, management recognised that Firm D had a significant market advantage associated with its assets and internal capabilities (P2):

I think our competitiveness lies in the ability to support customers' sustainability initiatives. We have gained a lot of voluntary carbon credits such as VERs from our renewable development. These credits can be sold to our existing corporate customers to help them achieve their carbon neutrality objectives. We also have significant knowledge in carbon trading, which we can share with our customers. (A Senior Manager)

Consequently, a marketing campaign was launched to reinforce its green brand and commitment with a purpose to enhance its carbon-related competitiveness (Various advertising materials) (P3). This campaign was believed by management to have attracted new corporate customers to the generator.

However, despite its announced commitment to sustainability, Firm D did not disclose the specific energy efficiency initiatives undertaken in this period (Annual Report 2009) (P4). It also failed to renew its CarboNZero certification for 2009 (P3). Furthermore, customer energy efficiency initiatives were reduced since management perceived that these initiatives had reduced Firm D's sales volume and exerted a negative impact on its short-term profitability (interview with a senior manager) (P3). The primary measure for the generator's environmental sustainability continued to be the level of compliance with RMA resource consents (Annual report and interview data) (P4). Similarly to the 
previous period, while claiming to follow GRI reporting guidelines, Firm D provided only superficial and descriptive discussion of its environmental performance (P4). It also failed to establish a company-wide emissions monitoring and reporting system in order to achieve the emissions reduction targets which were set as part of 2008 CarboNZero certification process (interview data) (P4). Therefore, Firm D's climate change initiatives were limited and narrow in scope, which contrasts with the climate change practices undertaken by Firm $\mathrm{C}$ which also claimed a commitment to sustainability. An interview with one internal manager suggests that this is partly due to Firm D's small size, which led its management to perceive a low exposure to institutional pressures and thus little need to employ extensive climate change initiatives to maintain social legitimacy.

\subsubsection{Summary}

In summary, the evidence presented from 6.5.1 to 6.5.4 above suggests that Firm D changed its environmental strategies and organisational MCS in response to GCCP changes. Table 6-4 provides the count of evidence related to Firm D that supports the four propositions.

Table 6-4: Count of Firm D-related evidence availability

\begin{tabular}{|l|c|c|c|c|}
\hline & Apr02 - Dec05 & Jan06 - Sep07 & Oct07 - Sep08 & Oct08 - Oct09 \\
\hline P1: Economic drivers & 2 & 4 & 5 & 2 \\
\hline \multicolumn{1}{|c|}{ Institutional drivers } & 1 & 1 & 0 & 0 \\
\hline $\begin{array}{l}\text { P2: Internal characteristics } \\
\text { and capabilities }\end{array}$ & 3 & 4 & 3 & 1 \\
\hline $\begin{array}{l}\text { P3: Change in } \\
\text { environmental strategies }\end{array}$ & 4 & 6 & 4 & 6 \\
\hline P4: Change in MCS & 4 & 6 & 5 & 6 \\
\hline
\end{tabular}

The table shows that Firm D perceived strong GCCP-related economic drivers from Jan2006 to Sep2008, when the ETS was developed and legislated. These drivers, including regulatory uncertainty, changing fuel economics, and international regulatory environment, had implications for its competitiveness (P1). In contrast, Firm D had limited exposure to institutional pressures throughout the whole period, possibly due to its small size and private ownership structure (2000-2009) (P1). Firm D also recognised early (from Jan2002) that it had a strong market advantage under the GCCPs because of its renewable-focused internal capabilities (P2). Consequently, since 2002, Firm D has made many changes to its environmental strategies and organisational MCS to reflect this advantage (P3, P4). Its environmental strategies were modified in areas relating to generation investment, retail, carbon neutrality and internal energy efficiency (P4). From a MCS perspective, to implement the strategic changes, Firm D integrated carbon 
cost in its generation investment decisions and set up control systems to facilitate carbon trading, to maximise its competitiveness. However, its emissions-related external disclosure was limited, corresponding to the low exposure to institutional pressures.

\subsection{Firm E: a renewable generator}

Firm E was one of the three SOEs established as the result of the separation of the ECNZ's generation assets in 1999 and currently is in state ownership. The generator inherited hydro and geothermal assets of which renewable-based generation accounted for $87 \%$ of its total capacity in 2000 . Therefore, it has a lower level of carbon emissions and associated carbon-related costs under the GCCPs than thermal generators (A and B), but more than other renewable generators (C and D). It acquired various retailing businesses between 1999 and 2001 and became vertically integrated, accounting for $15 \%$ of the total wholesale and retail electricity market share in 2003. Its generation plants are located in the North Island but its retail bases are in both the North Island and South Island. At establishment, generation development efforts were focused on hydro schemes. However, from 2002, it began to diversify into other fuels, including geothermal, gas and wind. Later on, Firm E refocused its generation investment strategy on geothermal energy only. The changes in its environmental strategies and organisational MCS in association with the changes in the GCCPS are presented below.

\subsubsection{January 2000 to March 2002}

During this period, before any GCCP was introduced, Firm E focused on hydro generation. Top management decided that in the future, its competitive advantage would be in renewable development (geothermal, small hydro and other renewable energy) (interview with a past manager) (P3). Additionally, Firm E was one of the first in the industry to discuss climate change and its impacts on electricity generation operations (Annual Report 2002). The generator emphasised that its current hydro-focused generation strategy was consistent with the Government's probable Kyoto position and that its planned renewable investments would provide significant climate change benefits.

The generator took a lead in encouraging customer energy efficiency in response to the energy shortage in winter 2001 (P3). It was the only retailer that offered customers payment for their reductions in energy consumption (Media release 18 December 2001). This is because its managers recognised that "energy efficiency benefits both Mighty River and the customer - they save money on their power bill and we conserve water for 
later use" (Stakeholder Newsletter, December 2001). However, no initiatives were undertaken to encourage energy efficiency internally (P3).

Initially, Firm E's sustainability policy had a strong focus on RMA compliance and there was no consideration of climate change issues in this policy (P4). However, the generator joined the NZBCSD, which exerted pressures on participants to adopt a sustainability-oriented business model (P1). As a result, Firm E reviewed its sustainability policy and sought ways to integrate sustainability into business decisions (P4). Further, it considered that "it is no longer appropriate to confine the picture of ourselves to just financial or economic performance" and that the company needs to be viewed from "across the spectrum of issues that impact on the way we do all our business" (Sustainability Report 2001). Sustainable development was seen as the key to "hold the community's license to operate" (Annual Report 2002). Environmental performance standards were set internally but not disclosed externally, as the company was in the process of consulting with different stakeholders on the appropriateness of the different performance measures that align with its sustainability reporting (Sustainability Report 2001). Instead, Firm E chose to disclose overall compliance with environmental regulation as the key indicator of its environmental performance (P4).

\subsubsection{April 2002 to December 2005}

With the announcement of the carbon tax in April 2002, Firm E undertook a strategic diagnosis of "climate change risks and opportunities", focusing predominantly on the opportunities (Annual Report 2003) (P1). It was stated:

We identified a number of opportunities with climate change, including green branding, renewable resource use, niche generation, innovative energy solutions and, further into the future, prospects such as the hydrogen economy, where our hydro generation plant provides immediate real advantages. (Annual Report 2003)

Firm E took advantage of the PRE scheme to lever its renewable investment (P1, P3). It was granted an entitlement to over one million carbon credits for its geothermal projects from 2003 to 2004 (MfE, 2009d). Similar to other renewable generators, the potential revenue from the sale of these credits enhanced the financial viability of its geothermal projects (P2). Further, to gain a competitive advantage in geothermal energy, the generator invested heavily in developing geothermal-related human and resource capabilities (P2). It recognised that "geothermal, as the only reliable source of 
renewable power, is crucial to offsetting the weather related risks associated with hydro and wind generation" (Annual Report, 2002). Thus, the bottom line of this strategic choice was to optimise the efficiency of generation plants and thus minimise organisational exposure to wholesale market volatility (P3). However, this strategy was enabled by firm-level capabilities, as evidenced by the following quote:

With long experience in geothermal generation, its recent creation of a worldclass team of geothermal specialists, and its healthy profits, it is confident that there is no company better placed to successfully undertake this exploration in New Zealand. (Financial Review 2003/2004)

Firm E also began to investigate wind and small hydro possibilities from 2005. Simultaneously, it pursued thermal projects to diversify its fuel choices and thus "manage the supply shortage risks associated with renewable generation" (P3). The generator entered a joint venture for gas exploration and decided to apply for resource consent to re-commission a coal-fired plant (P3). Despite enormous opposition from the public, it defended this decision on the grounds of the need for security of supply, the availability of coal reserves and cheap operating costs due to plant already existing (P2) (Financial Review 2003/2004). One manager interviewed indicated that the economic impact of the carbon tax was considered when making thermal investment decisions (P1).

Rather than merely conforming to EECA's pressures for energy efficiency, Firm E considered this a market opportunity and undertook industry-leading initiatives in the customer energy efficiency area (P3). Accordingly, a substantial budget was committed to developing and marketing smart metering and energy efficiency products ( $\mathrm{P} 4)$. Furthermore, supported by top management commitment to sustainability, the environmental policy was changed to reflect an aim "to become a leader in sustainable development” (Sustainability Report 2004) (P2; P4). Managers suggested this new policy enabled the generator to realise a "green branding" market opportunity and distinguish itself from other electricity firms (Interview with a senior manager). However, this policy was not delegated down to the business unit level, where each unit maintained a separate environmental policy. Consistent with its sustainability policy, Firm E's political strategy focused on discussing and proposing further sustainability initiatives (Submissions to various draft regulations and bills) (P3). To protect its thermal interests, the generator also argued that fossil fuels were still needed to ensure a reliable electricity supply (P3). 
Interview data with internal managers suggest that Firm E's MCS was modified to support its environmental strategies. To realise its environmental policy to become a leader in sustainable development, the generator decided to set an industry standard for the internal EMS. Therefore, the Enviro-Mark Standard was used to design the EMS and associated environmental measures which enabled environmental performance to be managed (P4). Top management used the human resource management system interactively to identify and develop internal human skills and expertise related to sustainable development (P4). Furthermore, the generator developed a comprehensive MCS for managing internal energy efficiency and non-generation emissions. Crossfunctional teams were established to set environmental measures and manage environmental performance for each department/business unit (P4). Reduction targets were set for resource consumption in the component areas (i.e. waste and staff travel) that made up non-generation emissions, but no target was specifically determined for total non-generation emission levels (P4). Further, an environmental intranet was established to communicate non-generation emissions levels at an organisationally-wide level and monitor the achievement of the reduction targets (P4). This intranet was also used extensively to raise climate change awareness and encourage behaviour change (P4). These energy efficiency initiatives and MCS changes resulted in the generator receiving a number of external environmental awards (P4), as well as reinforcing an organisational culture of environmental responsibility (P2). The receipt of these awards and the environmentally-focused culture were heavily advertised by Firm E, thus enhancing its market reputation.

Between 2002 and 2005, there was a significant increase in emissions-related disclosure and a move from a compliance-focused environmental reporting to voluntary reporting of non-generation emissions. Additionally, Firm E was among the first in the industry to disclose its generation and non-generation emission levels in detail (Annual Reports 2004 and 2005). Generation carbon emissions were disclosed in the ARs as totals and by type and plant, while non-generation emissions were disclosed for the retail business, in total and broken down into the separate activity areas (P4).

\subsubsection{January 2006 to September 2007}

From early January to December 2006, due to the cancellation the carbon tax, Firm E decided to pursue a diversification generation strategy. Hence, it expanded its generation development activities across a wide range of fuel sources, both renewable and thermal-based. However, due to its internal geothermal expertise, geothermal 
development remained the "core strength" and strategic focus of its generation portfolio (Financial Review 2006/2007) (P2, P3).

From early 2007, as it became increasingly clear that the Government was developing an ETS, Firm E decided to narrow its strategic focus. It reduced gas exploration and wind development activities, discontinued the coal-fired project, and focused its investment programme on geothermal energy (P3). The scale-back of gas exploration was caused by the low probability of finding gas to support the generator's gas-fired plants (P2), while the delay in its wind development projects was associated with high increases in wind turbine prices (P1) (interview data and Annual Report 2007). However, the cancellation of the coal-fired project was potentially not driven by a purecost rationale. A Greenpeace New Zealand interviewee indicated that a possible reason for cancellation was strong public opposition to coal-fired generation (P1). Firm E itself acknowledged that "climate change implications have made coal an undesirable source of generation" (Annual Report 2007). However, it defended it decision on an economic basis:

Since that decision [apply for resource consent of the coal-fired plant] was made, we have commissioned additional geothermal capacity and successfully consented and begun construction on other projects... As a result of this work and other developments within the industry we no longer see scenarios where Marsden B will be cost effective in the next decade or so. (Media Release 7 March 2007)

]Following this coal-fired plant cancellation, the generator placed increased importance on carbon footprint considerations when evaluating future investment projects (P1) (interview with a senior manager). Therefore, in subsequent governmental submissions and public documents, it emphasised that its emissions management strategy was to develop renewable power stations (P3). This strategy was also undertaken because the company perceived little discretion in controlling the emission levels from its existing generation plants:

The aggregate level of emissions is a function of demand and hydrology. If there is an extended dry or calm (wet or windy) spell, emissions from our gas fired station will increase (decrease). Our other stations with lower emission intensity run baseload and are unaffected by the weather. As Mighty River Power does not control the weather we are unable to affect changes in gas fired station emissions. (Financial Review Questionnaire 2006/2007) 
Firm E's political activity during 2006-2007 focused around the draft NZES. It lobbied strongly against the NZES sole focus on emission reductions and argued it was unfair to place the burden of emissions reduction only on the electricity sector (P3). Using accounting-based analysis, the generator cautioned that the Government's ambitious emissions reduction target would require impossible achievements in renewable generation development and would put security of supply at risk (Submission to draft NZES) (P4). Additionally, the generator gave conflicting messages to the Government regarding a possible change in its retail strategy as a result of a potential ETS (P3). In its response to Financial Review Questionnaire 2006/2007, it underplayed the impact of carbon charge on the retail prices, stating that "we will only set prices sufficient to support our investments". However, in its submission to the draft NZES, the generator admitted that an ETS would significantly influence its price setting decisions, but did not provide a quantitative estimate of the influence (P1).

Interviews with managers indicate that organisational MCS underwent significant changes internally. To ensure compliance with complex resource consent conditions, Firm E installed a continuous generation emissions monitoring and reporting system as part of the EMS (P4). A new measure, generation emissions intensity, was adopted to monitor the carbon footprint of generation plants using different fuel types (P4). However, no targets or specific initiatives were implemented to reduce generation emissions or emissions intensity. For non-generation emissions, a new whole-ofcompany environmental policy was developed to replace the individual environmental policies of each business unit (P4). Consequently, a central team was established to replace the previous cross-functional teams and non-generation emissions were monitored across the whole company (P4). A budget was assigned to support the implementation of climate change initiatives (P4). Additionally, the EMS in all business units attained Diamond status for the Enviro-Mark Standard, making Firm E the only New Zealand energy company to have the whole organisation certified to this high standard by an external agency (P4). The achievement of this certification was heavily advertised as the evidence of its commitment to environmental excellence.

Externally, environmental disclosure was integrated in the ARs and gained a new label, "Climate change, energy efficiency and renewables", which aligned with the dominating themes of the Labour Government's GCCP at that time (P4). However, the level of external disclosure related to generation emissions decreased compared to the previous period (P4). A Senior Manager explained that its low emission profile drove this reduction $(\mathrm{P} 2)$ : 
Compared with the other thermal generators, we have a lot lower emission level. So we feel that we are exposed to low public pressures with respect to managing and disclosing generation emissions. On top of that, we choose to focus efforts on increasing renewable generation, rather than managing existing emissions...

\subsubsection{October 2007 to September 2008}

Once the Original ETS Bill was released for public consultation in December 2007, Firm E undertook further active lobbying activities (P3). In its submission, the generator supported the ETS and the opt-in flexibility for gas and coal purchasers to directly manage their emissions, but opposed the thermal ban. Using accounting-based analysis, it also argued for the exclusion of emissions from geothermal fields, thus reducing its potential ETS liabilities and compliance costs (P3, P4). These arguments were primarily economically-driven, based on its ownership of both renewable and thermal generation assets (P2). Its generation investment strategy remained focused on geothermal development (P3). Additionally, it increased its stake in a wind turbine company as wind generation had become more economic due to the ETS's carbon charge on fossil fuels (P1, P3).

Building upon its previous experience in customer energy efficiency (P2), Firm E established a separate smart metering business and launched a multi-million dollar retail campaign to promote smart meters (Annual Report 2008) (P3). This was acknowledged as a strategic move to capture the market opportunities associated with increasing demand from domestic customers for products that monitored consumption and allowed them to manage their energy costs (P1). Internally, energy efficiency initiatives undertaken in previous periods were continued, with a particular focus on encouraging recycling and reducing waste to landfills (Interview data) (P3). In contrast to the thermal generators, Firm E did not plan to purchase carbon credits to prepare for its future ETS compliance obligations (P3). Analysis of public documents indicates that the generator's carbon credits granted under the PRE scheme were more than those needed to satisfy its ETS obligations (due to its low level of generation emissions) (P2). Additionally, managers perceived that managing existing generation emissions was difficult because the use of thermal plants and associated emissions levels were driven by uncontrollable factors, e.g. external weather conditions (P2, P3). A calculation of the emissions intensity disclosed in annual reports indicates Firm E's generation emissions intensity fluctuated significantly because of year-to-year changes in hydro levels. 
Consequently, the measure of generation emissions intensity adopted in the previous period was dropped in 2008 (P4). Instead, top management focused on measures of total emissions and the carbon cost avoided from new renewable development (Interview data) (P4).

Interviews with managers also suggest that carbon prices and external fuel economics were monitored and integrated in investment planning systems (P4). As a result, the plan for an ETS had discouraged thermal investments, even though the ETS had not been introduced (Submission to Original ETS Bill) (P3). Firm E also established a database to monitor changes in customer demand and expectations and used this database extensively to enable coordination between different functions in implementing its smart-metering retail campaign (Interview data) (P4).

In contrast to the other generators, Firm E decided not to pursue CarboNZero certification (P3). Interviews and documents suggest that top management considered such certification programmes as "snake oil", a tool to avoid responsibility without direct and open consultation and engagement with external stakeholders and wider community to solve the real problems. Instead, the generator announced its commitment to sustainability was born by "many years of maintaining the highest status of the Enviro-Mark external accreditation" for its EMS and environmental initiatives (Annual Report 2008) (P4). Furthermore, emissions-related external disclosure improved, with total emissions being reported publicly for both generation and non-generation activities (P4).

\subsubsection{October 2008 to October 2009}

The change of Government in late 2008 delayed the introduction of the Original ETS. Management foresaw this delay and other proposed changes to the ETS, for example, the carbon price cap (Interview data) (P1). As a result, Firm E's political activity was substantially curtailed and it chose not to participate in the policy process related to the Moderated ETS (P3). Senior managers perceived that the lack of political consensus between the two main political parties would be a disincentive to carbon management activity and renewable generation investments (Interview data) (P1). However, the generator did not place on hold its investments in renewables. In contrast, it established a global geothermal strategy and committed a substantial budget to the expansion of its geothermal business overseas (Media Release 19 November 2008) (P3, P4). This strategy was timed to capture the market opportunities brought about by changing international regulatory environments in favour of renewable energy (P1). Further, the 
strategy was feasible, as Firm E was a market leader in geothermal energy because of prior geothermal investments and the previous development of human capabilities and expertise in this area (P2). One Manager commented:

The extensive geothermal development programme is supported by significant investment decisions in exploration we made years before... It is a courageous approach, but one that we have the people, expertise, experience and foresight to make... In total, our people have worldwide experience in more than 30 developed geothermal fields across 10 countries and more than 100 exploration projects. This experience and geothermal know-how is invaluable and underlines our commitment as New Zealand's number one geothermal operator.

It was revealed that Firm E's exposure to future carbon emissions costs was "limited" due to the "90\% renewable portfolio" (Annual Report 2009) (P2). A carbon credit strategy was established in which the PRE carbon credits would be used for both compliance and trading purposes (P2). This strategy also focused on building internal capabilities in carbon trading to enable effective management of ETS carbon-related costs and revenue (P3). Its retail strategy in this period continued to focus on customer energy efficiency and smart metering products and services (P3).

Interview data indicate that to support its global geothermal strategy, top management used the HRM system more interactively to expand the geothermal expertise and skill base among the employees (P4). Consistent with its carbon credit strategy, formal controls and control systems were established to measure, assess and manage its ETS compliance costs and exposure (P4). Additionally, Firm E planned to reduce and mitigate its carbon costs through technological innovation, changing management practices, and accumulating carbon credits (P3). To implement this plan, internal carbon trading human and non-human capabilities were developed (P3). The generator also decided to outsource carbon trading transactions that required complex knowledge and expertise that was not available among existing staff (P3). A carbon trading information system was set up to monitor changes in carbon price to assess the impact of such changes on the generator's ETS emissions costs (P4). The emissions monitoring system was assigned more importance as it provided information for monitoring emissions levels and the initiatives undertaken to reduce and offset emissions (P4). Further, a climate change risk register was established in which carbon-related risks and opportunities were identified, and their impacts evaluated and monitored (P4). The monitoring of these risks and opportunities was assigned to the existing trading 
platforms and associated systems that were part of the wholesale trading department. Additionally, inter-system linkages were established between the databases of different functions and departments to ensure that managers could acquire relevant carbon-related information for their decision-making needs (P4).

Under performance pressures from the Government (P1), Firm E restructured internally to achieve efficiency gains in its operations and activities (P3). Interview data indicate that this restructuring resulted in less financial resources being dedicated to internal energy efficiency areas (P2). Consequently, organisational MCS related to internal energy efficiency, such as the non-generation emissions monitoring system and environmental intranet, was used less at strategic and operational levels (P4). Nonetheless, due to the need to prepare for the upcoming ETS enforcement, the emphasis on generation emissions monitoring and carbon trading activities was not reduced $(\mathrm{P} 4)$.

In this period, there was an increase in the amount of emissions-related disclosure, as Firm E's waste and electricity use began to be measured and reported (P4). However, it divided external disclosure between the AR and the company website (P4). The 2009 $\mathrm{AR}$ included the aggregate measures of generation and non-generation emission, while the website listed the break-down of generation measures by fuel type and of nongeneration measures by activity (P4). Firm E also claimed to follow the GRI guidelines in preparing the sustainability content in its 2009 AR (P4).

\subsubsection{Summary}

In summary, the evidence presented above suggests that Firm E's environmental strategies and organisational MCS changed from 2000 to 2009 in accordance with GCCP changes. Table 6-5 summarises the count of evidence related to Firm E that support the four propositions.

Table 6-5: Count of Firm E-related evidence availability

\begin{tabular}{|c|c|c|c|c|c|}
\hline & Jan00 - Mar02 & Apr02 - Dec05 & Jan06 - Sep07 & Oct07-Sep08 & Oct08-Oct09 \\
\hline $\begin{array}{l}\text { P1: } \\
\text { Economic drivers }\end{array}$ & 0 & 3 & 3 & 2 & 3 \\
\hline Institutional drivers & 1 & 1 & 1 & 0 & 1 \\
\hline $\begin{array}{l}\text { P2: } \\
\text { Internal characteristics } \\
\text { and capabilities }\end{array}$ & 0 & 3 & 3 & 4 & 4 \\
\hline $\begin{array}{l}\text { P3: Change in } \\
\text { environmental } \\
\text { strategies }\end{array}$ & 3 & 6 & 5 & 7 & 8 \\
\hline P4: Change in MCS & 3 & 10 & 9 & 8 & 12 \\
\hline
\end{tabular}


The table suggests that Firm E perceived little institutional pressures from the GCCPs. Those that did exist were predominantly societal pressures due to its generation emission profile (P1, P2). However, Firm E recognised the potential implications of GCCP-related external economic drivers as early as Apr2002, when the carbon tax was announced (P1). These drivers, including changing fuel economics, regulatory uncertainty, and international regulatory environment, affected Firm E's competitiveness and carbon costs. Simultaneously, Firm E realised that its renewablefocused internal capabilities would enable it to gain a competitive advantage under the GCCPs (P2). Consequently, since 2002, Firm E has made consistent changes to its environmental strategies to match the changing environment (P3). The key strategic changes included a modification in its generation investment and retail strategies, and the adoption of customer and internal energy efficiency initiatives (P3). Many organisational MCS changes were also made from 2002, primarily to support these competitiveness-driven environmental strategies (P4). The highest number of MCS changes was implemented from Oct2008 to Oct2009, as Firm E prepared for the management of its carbon costs under the upcoming ETS (P4).

\subsection{Summary}

To summarise, this chapter has provided case-based evidence of changes in environmental strategies and organisational MCS made within the five electricity generators in response to GCCP changes. Where applicable, support for P1, P2, P3 and P4 has been indicated. Figure 6-1 summarises the evidence for the four propositions across the five generators as presented from sections 6.2 to 6.6 above. It should be noted that the numbers illustrated in this figure represent the count of evidence relating to the propositions, rather than the degree of impact exerted by external and internal drivers or the scope or extent that strategic and MCS changes were undertaken, which are to be analysed and discussed in Chapters 7 and 8. Additionally, the count of evidence indicates the number of new external and internal drivers recognised by a generator in a given period (compared to the previous period), the number of environmental strategy changes and MCS changes made in response to these new drivers. 
Figure 6-1: Count of evidence of change across the five generators

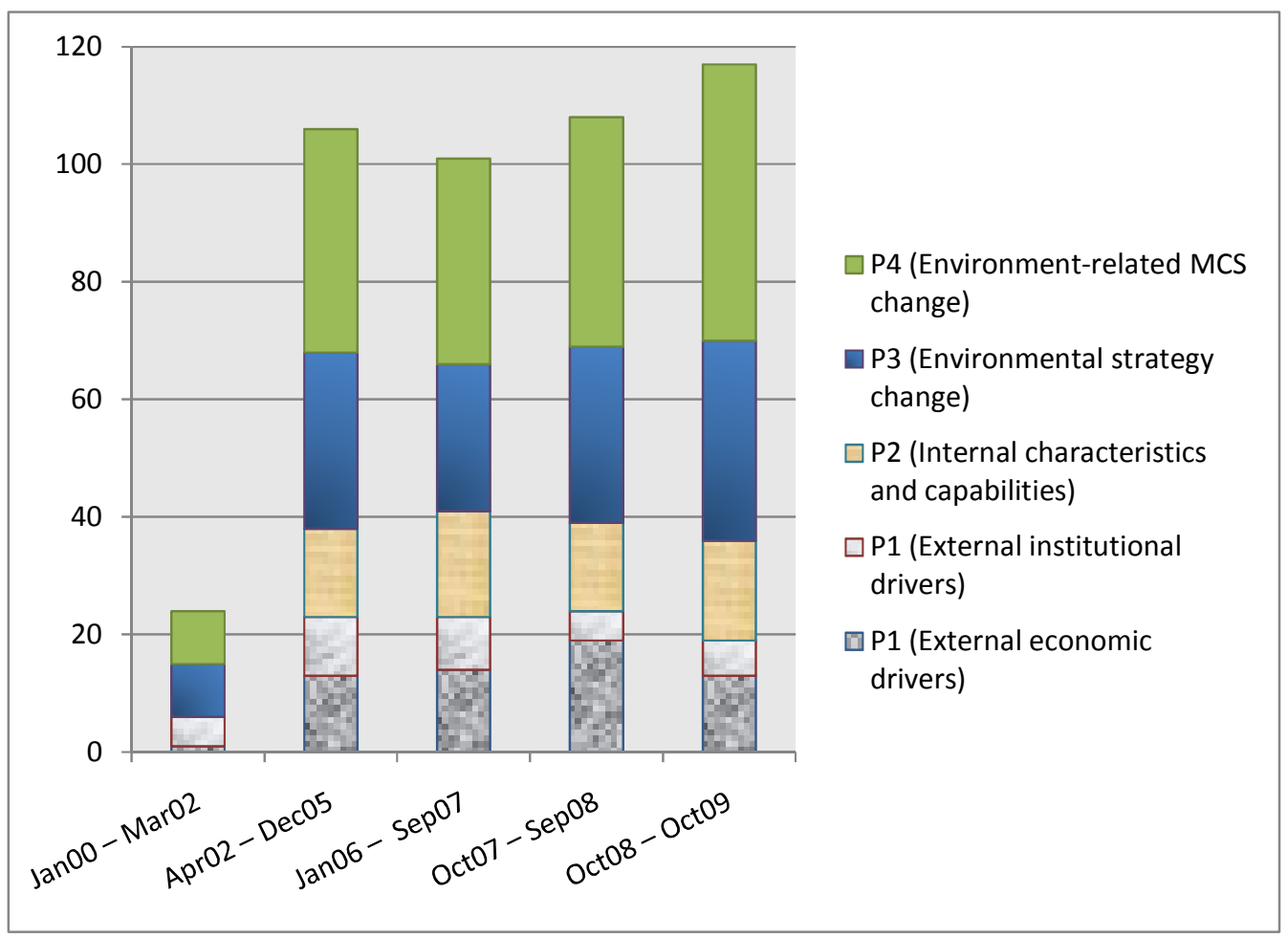

In the absence of any GCCP, there was little recognition of potential climate changerelated impacts from January 2000 to March 2002, leading to few changes in environmental strategies and organisational MCS. The five generators perceived the highest number of new institutional pressures during the carbon tax period from April 2002 to December 2005, as the society started to become aware of climate change issues $^{7}$. In contrast, these generators recognised an increasing number of additional economic drivers between the carbon tax period (Apr2002-Dec2005) and the Original ETS development and legislation period (Jan2006-Sep2008), as the diagnosis was undertaken within these organisations to understand the potential economic impacts of the GCCPs. However, the number of changes in external economic drivers decreased between October 2008 and October 2009, mostly due to the policy and regulatory uncertainty associated with the ETS review (P1). Additionally, since the carbon tax announcement, generators recognised that internal characteristics and capabilities had implications for their organisational exposure to the GCCPs, which in turn affected the choice of their environmental strategies (P2, P3).

\footnotetext{
${ }^{7}$ A lower count of evidence related to institutional pressures in the subsequent periods does not indicate that generators perceived less exposure to institutional pressures. Instead, the count only highlights that generators perceived a lower number of new types/sources of institutional pressures. The pressures recognised in the previous periods might still apply, and the degree of their impacts on the generators might increase over time, e.g. the case with societal pressures associated with emission reductions.
} 
The generators made the most changes to their environmental strategies during the periods of certainty in the GCCPs, Apr2002-Dec2005 and Oct2007-Oct2009 (P3). This is potentially because the impacts of external GCCP-related drivers on the organisation were clear during these periods and hence required appropriate strategic responses. To implement these strategic changes, the generators made many modifications to their organisational MCS within the same periods (P4). The highest number of changes to MCS design and use were observed during the last period (Oct2008-Oct2009), as the generators prepared organisationally for the upcoming ETS enforcement (P4).

The next chapter analyses the longitudinal evidence presented above to understand the impacts of the GCCP-related external and internal drivers on environmental strategies across the five generators and the variations in strategy proactiveness and strategic emphasis pursued by these generators in response to the GCCPs (P1, P2, and P3). Following this, Chapter 8 will analyse the evidence related to organisational MCS change to gain insights into the modifications in MCS design, use, and objectives across the five generators to support the environmental strategic changes (P4). 


\section{Chapter 7: GCCP-related external and internal drivers and their impacts on environmental strategies}

\subsection{Introduction}

Chapter 6 provided the evidence regarding the changes in environmental strategies and associated organisational MCS within each of the five generators from 2000 to 2009. It also highlighted how such evidence supports the four propositions on an individual case basis. The main aim of Chapter 7 is to provide a cross-case empirical analysis in order to validate the first three propositions that relate to GCCP-related external and internal drivers and environmental strategies (P1, P2, and P3). Where applicable, it will draw out new insights that are not captured in the propositions or the research framework. Accordingly, this chapter analyses the evidence presented in Chapter 6, identifies the possible impacts of the GCCP-related external and internal drivers on the generators and examined how they changed their environmental strategies in response. The results presented in this chapter are gained from a comparative analysis of the qualitative evidence presented in Chapter 6 and other relevant interview and documentary data, the coding and analysis of which is discussed in Chapter 5. The tables and figures (i.e. charts) provided are derived from Excel-based data matrix displays (5.7.2). ${ }^{8}$ Where applicable, a reference to a section in Chapter 6 will be made to highlight the source of evidence that supports a key argument or finding (e.g. 6.2.1).

The rest of the chapter is organised into six sections. The next section provides an overview of the five generators' characteristics and the types of environmental strategies adopted in response to the GCCPs. The external volatilities and pressures and their impacts on environmental strategies are identified and examined next. Following this, results related to the influence of GCCP-related internal characteristics and capabilities on environmental strategies by different generators are presented. The results to changes in environmental strategies, as reflected by the level of strategy proactiveness and strategic emphasis, are discussed next. This is followed by the analysis of the objectives motivating such environmental strategic change. The chapter is concluded with a summary.

\footnotetext{
${ }^{8}$ These matrix displays are available on request provided they do not breach the confidentiality agreements with the interviewees
} 


\subsection{Overview of the five generators and the types of environmental strategies}

Table 7-1 provides a comparative overview of the key internal characteristics of the five generators using data from publicly available documents. This table confirms that there is significant diversity and variation in the internal characteristics of the five generators, which will be examined in more detail in section 7.4. Understanding these internal characteristics facilitates the explanation of the drivers of the change in generators' environmental strategies in a GCCP-focused context.

Table 7-1: Demographics of the five generators

\begin{tabular}{|c|c|c|c|c|c|}
\hline \multirow[b]{2}{*}{$\begin{array}{l}\text { FIRM-LEVEL } \\
\text { CHARCTERISTICS }\end{array}$} & \multicolumn{2}{|c|}{ Thermal generators } & \multicolumn{3}{|c|}{ Renewable generators } \\
\hline & Firm A & Firm B & Firm C & Firm D & Firm E \\
\hline Ownership structure & $\begin{array}{l}\text { Publicly- } \\
\text { listed }\end{array}$ & State-owned & State-owned & $\begin{array}{l}\text { Publicly- } \\
\text { listed }\end{array}$ & State-owned \\
\hline \multicolumn{6}{|l|}{ Generation } \\
\hline Plant location & NI & NI & $\mathrm{NI}+\mathrm{SI}$ & $\mathrm{NI}+\mathrm{SI}$ & NI \\
\hline Generation sources (2002)* & $\begin{array}{c}\text { Thermal: } \\
63 \% \\
\text { Renewable: } \\
36 \%\end{array}$ & $\begin{array}{c}\text { Thermal } \\
67 \% \\
\text { Renewable } \\
33 \%\end{array}$ & $\begin{array}{c}\text { Renewable: } \\
100 \%\end{array}$ & $\begin{array}{c}\text { Renewable: } \\
100 \%\end{array}$ & $\begin{array}{c}\text { Renewable: } \\
87 \% \\
\text { Thermal } \\
13 \%\end{array}$ \\
\hline $\begin{array}{l}\text { Integration between } \\
\text { generation and retail bases }\end{array}$ & Net seller & Net seller & Net seller & Net buyer & Net seller \\
\hline \multicolumn{6}{|l|}{ Retail } \\
\hline Retail areas & $\mathrm{NI}+\mathrm{SI}$ & NI & $\mathrm{NI}+\mathrm{SI}$ & $\mathrm{NI}+\mathrm{SI}$ & $\mathrm{NI}+\mathrm{SI}$ \\
\hline $\begin{array}{l}\text { Retail market share } \\
\text { (customer number) (2003)** }\end{array}$ & $26 \%$ & $25 \%$ & $13 \%$ & $16 \%$ & $15 \% *$ \\
\hline \multicolumn{6}{|l|}{ Emissions profile (2008) } \\
\hline $\begin{array}{l}\text { Generation emissions } \\
\text { (ktonnes) }\end{array}$ & 2100 & 3500 & 0 & 0 & 591 \\
\hline $\begin{array}{l}\text { Non-generation emissions } \\
\text { (ktonnes) }\end{array}$ & 3.5 & 1.0 & 5.8 & 2.8 & 1.6 \\
\hline \multicolumn{6}{|l|}{ Organisational size (2008) } \\
\hline Number of employees & 1,000 & 534 & 568 & 455 & 752 \\
\hline Total assets (mil NZ\$) & 5,203 & 2,707 & 7,197 & 2,351 & 4,058 \\
\hline $\begin{array}{l}\text { Wholesale market share } \\
\text { (Gwh) (2003) }\end{array}$ & $23 \%$ & $19 \%$ & $29 \%$ & $5 \%$ & $15 \% * *$ \\
\hline
\end{tabular}

Key: NI: North Island; SI: South Island

*: to preserve anonymity of the case generators, it is considered appropriate not to disclose the exact proportions of the different types of fuels that make up a generator's total generation capacity.

**: the market shares of the five generators do not add up to $100 \%$. There are other small generators and retailers which make up $9 \%$ and $5 \%$ of the generation and retailing market shares respectively.

Further, the analysis of the evidence from Chapter 6 suggests that eleven different environmental strategies were used by the generators to respond to the GCCPs. Some of these strategies were already adopted before any GCCPs, such as the generation (investment) strategy, retail strategy, political strategy, environmental/sustainability policy, and these were modified when the GCCPs were announced or introduced. Other environmental strategies were formulated specifically to respond to the volatilities and 
pressures associated with the GCCPs. They include internal energy efficiency, customer energy efficiency, carbon credit, carbon neutrality, emissions management and emissions-related disclosure strategies and internal restructuring. The eleven environmental strategies are influenced to varying degrees by the GCCP-related external and internal drivers identified through the interviews and documentary data related to the five generators (Chapter 6). The eleven strategies are listed and coded in Table 7-2.

Table 7-2: Coding for environmental strategies

\begin{tabular}{|l|l|l|l|}
\hline GI & Generation (investment) strategy & EP & Environmental/sustainability policy \\
\hline RS & Retail strategy & EM & Emissions management strategy \\
\hline IEE & Internal energy efficiency & ED & Emissions-related disclosure strategy \\
\hline CEE & Customer energy efficiency & CN & Carbon neutrality strategy \\
\hline CC & Carbon credit strategy & PS & Political strategy \\
\hline IR & Internal restructuring & & \\
\hline
\end{tabular}

\subsection{External GCCP-related drivers}

This section addresses P1 \& P3 by presenting the findings related to the external volatilities and pressures associated with the various GCCP changes from 2000 to 2009. Specifically, how each GCCP-related external volatility and pressure (driver) was applied to or perceived by each generator and how it influenced generators' environmental strategies are examined. Then, all the drivers are combined and quantified in a matrix data display to represent the degree of total external exposure of each generator to the GCCPs. For the purpose of this study, the degree of total external exposure represents the aggregate degree of impacts exerted by all the GCCP-related external drivers on each generator.

Most of the external volatilities and pressures are related to all the GCCPs, including the carbon tax, the PRE scheme, the proposed thermal ban, and the ETS. However, some emerged from a specific GCCP, as indicated by the brackets ( ) in Tables 7-3 and 7-4. To enhance the clarity of the analysis, the volatilities and pressures are separated into Economic and Institutional ones. External economic volatilities and their impacts on the generators' environmental strategies are analysed first.

\subsubsection{Economic volatilities}

External GCCP-related economic volatilities and pressures are classified into four categories: policy and regulatory change, changing fuel economics, carbon market fluctuations, and changing competition, as summarised in Table 7-3. The first three 
categories are consistent with economic drivers in the Research Framework presented in Figure 4-2 while the last group - changing competition is an additional group identified through the analysis of interview and documentary data.

\section{Table 7-3: External economic volatilities and their impacts on environmental strategies (P1, P3)}

\begin{tabular}{|c|c|c|c|}
\hline \multicolumn{2}{|c|}{ External economic volatilities } & $\begin{array}{l}\text { Apply in } \\
\text { generator }\end{array}$ & $\begin{array}{c}\text { Influence on } \\
\text { strategy }\end{array}$ \\
\hline \multirow{4}{*}{$\begin{array}{l}\text { Policy and } \\
\text { regulatory } \\
\text { change }\end{array}$} & Regulatory uncertainty and political instability & All & GI, PS \\
\hline & \multirow{2}{*}{ ETS delay and cap on carbon price (ETS) } & All & PS \\
\hline & & $\mathrm{A}, \mathrm{C}, \mathrm{D}$ & GI \\
\hline & International regulatory environment & $\mathrm{C}, \mathrm{D}, \mathrm{E}$ & GI \\
\hline \multirow{3}{*}{$\begin{array}{l}\text { Changing } \\
\text { fuel } \\
\text { economics }\end{array}$} & Renewable investment encouragement (PRE) & $\mathrm{B}, \mathrm{C}, \mathrm{D}, \mathrm{E}$ & $\mathrm{CC}, \mathrm{GI}$ \\
\hline & Increase in wholesale prices (ETS) & All & GI \\
\hline & External technological development & $\mathrm{A}, \mathrm{C}, \mathrm{E}$ & GI \\
\hline \multirow{2}{*}{$\begin{array}{l}\text { Carbon } \\
\text { market } \\
\text { fluctuations }\end{array}$} & Carbon price fluctuations (ETS) & All & $\mathrm{CC}$ \\
\hline & $\begin{array}{l}\text { Development of carbon trading expertise based } \\
\text { on PRE carbon credits (PRE) }\end{array}$ & $\mathrm{B}, \mathrm{C}, \mathrm{D}, \mathrm{E}$ & $\mathrm{CC}, \mathrm{RS}$ \\
\hline \multirow{3}{*}{$\begin{array}{l}\text { Changing } \\
\text { competition }\end{array}$} & Changing retail competition (green branding) & All & $\mathrm{RS}, \mathrm{CN}$ \\
\hline & $\begin{array}{l}\text { Carbon trading competition from thermal } \\
\text { generators }\end{array}$ & $\mathrm{C}, \mathrm{D}$ & $\mathrm{CC}$ \\
\hline & Energy efficiency market opportunities & $\mathrm{A}, \mathrm{B}, \mathrm{C}, \mathrm{E}$ & CEE, RS \\
\hline
\end{tabular}

\section{Policy and regulatory change}

Regulatory uncertainty surrounding various changes in the GCCPs and political instability due to the lack of consensus between the two major parties (National and Labour) on GCCPs from 2002 to 2009, represented major external pressures for all generators. Such uncertainty and political instability were perceived to indicate a lack of a long-term commitment to a carbon charge by the Government. This in turn creates ambiguity about the level of carbon-related costs generators would incur under a given GCCP. Furthermore, the GCCP changes led to uncertainty about the viability of alternative fuels and thus the competitiveness of the generators that strategically focused on a particular fuel type (renewables or thermals). Consequently, such uncertainty resulted in delays or disruptions to new generation investment decisions (e.g. 6.2 .5 \& 6.4.5). Many generators undertook a political strategy to participate actively in the GCCP policy processes (e.g. 6.2.4 \& 6.3.4). This participation not only helped them understand potential policy and regulatory changes but also allowed them to potentially influence policy design to maintain their own organisational advantages.

The ETS delay and the National Government's decision to put a cap on carbon prices in 2008/2009 were perceived to reduce the attractiveness of renewable sources and hence 
affect the competitiveness of different generators. In turn, such policy changes significantly influenced the generators' generation investment and political strategies. Three generators (A, C, D) proceeded either cautiously with their renewable investments, or put them on hold (e.g. 6.2.5 \& 6.4.5). Nonetheless, because all generators had anticipated these ETS changes before they were announced, they reduced their political activity in response. ${ }^{9}$

Renewable-based Firms C, D and E also perceived opportunities within the international regulatory environment which increasingly supports renewable generation since such environment had the potential to enhance these generators' competitiveness $(6.4 .2,6.5 .2$ $\&$ 6.6.5). Furthermore, these generators had substantial expertise and economies of scale in a specialised type of renewable energy (wind and geothermal) which enabled them to expand their renewable investments overseas to take advantage of this changing international regulatory environment.

\section{Changing fuel economics}

Changes in the relative economics between alternative fuels for electricity generation were perceived as one of the most significant external volatilities for all generators. These changes were caused by three factors: i) renewable investment encouragement through the PRE scheme (2003-2005), ii) increase in wholesale electricity prices due to the ETS (from September 2007), and iii) external technological development. These factors had implications for generators' production costs and revenue (competitiveness).

All generators, except Firm A (thermal, privately-owned), utilised the Government's PRE scheme to gain carbon credits associated with their proposed renewable investments ${ }^{10}$. The potential revenue from selling these carbon credits enhanced the financial viability of renewable projects, making them as desirable as, or even more attractive, than thermal projects. The PRE scheme thus contributed to a shift in the generators' generation strategy towards renewable sources.

Impacts of an increase in wholesale prices due to the ETS were also perceived by all five generators (e.g. 6.2.3, 6.4.4., \& 6.5.3). Within the New Zealand electricity markets, all generators are paid (every half an hour) the same wholesale price set by the most expensive generation. Under an ETS, the wholesale prices will therefore reflect the carbon costs of thermal generators, thus leading to significant windfall gains for

\footnotetext{
${ }^{9}$ See Sections 6.2.5; 6.3.5, 6.4.5, 6.5.4, and 6.6.5.

${ }^{10}$ Firm A perceived significant uncertainty with carbon pricing and potential carbon revenue and thus did not participate in the PRE scheme to gain carbon credits (6.2.2).
} 
renewable generators. Additionally, since such wholesale price increase will make renewable generation relatively more efficient than thermal generation, all five generators revised their investment strategies to put a stronger focus on renewables.

Furthermore, external technological development was perceived by three generators (A, $\mathrm{C}$ and $\mathrm{E})$ as an incentive to large-scale renewable investments (e.g. 6.4.3). Technological developments have improved the operational efficiency of renewable plants, reducing their production costs, and therefore enhancing the viability of renewable investments.

\section{Carbon market fluctuations}

Fluctuations in carbon prices are also a significant external volatility for all the generators. For generators who sold the carbon credits gained from the PRE scheme and voluntary emissions reduction standards $(\mathrm{C}, \mathrm{D}$, and $\mathrm{E})$, interview data suggests such fluctuations affected their ability to sell carbon credits at premium prices or find appropriate buyers, thus influencing their competitiveness. Generators that need to acquire carbon credits to meet their ETS obligations (A, B, and E) will be exposed to variations in the credibility of carbon credit contracts and increases in carbon prices which would make their purchases more costly (6.2.5).

Furthermore, for some generators (B, C, D and E), owning PRE-related carbon credits enabled them to take part in carbon markets to develop carbon trading skills ahead of the ETS (e.g. 6.4.4). They can use these skills to reduce their compliance carbon costs when the ETS comes into force, or to maximise revenue from carbon credit sales (carbon credit strategy). The generators also believed they can deploy these skills to assist industrial and business customers in meeting the latter's ETS compliance obligations or sustainability objectives (6.5.4). By doing so, generators' retail competitiveness is improved.

\section{Changing competition}

All generators also recognised that greening consumer expectations and public pressures associated with the GCCPs were changing retail competition within the electricity markets. To take advantage of this changing competitive environment, two $100 \%$ renewable-based generators $(\mathrm{C}$ and $\mathrm{D})$ extensively marketed and differentiated themselves as "clean and green" electricity suppliers (6.3.3 \& 6.5.4). The green branding and marketing campaigns undertaken by these renewable generators, to some extent, damaged the thermal generators' reputation (A, B and E) and reduced their market competitiveness. Furthermore, due to the cost of carbon, thermal generators 
might not be able to set retail charges at a competitive level (6.2.4). Thus, the changing retail market was perceived to affect the relative competitiveness between the five generators and thus the retail strategies they pursued. Changing retail competition also motivated some generators (A, C, D) to pursue carbon neutrality programmes to demonstrate their environmental responsibility in a GCCP-focused environment (6.2.4; $6.4 .3 ; 6.5 .3)$.

Additionally, two renewable generators ( $\mathrm{C}$ and $\mathrm{D})$ experienced a different kind of competitive pressures. They do not have a compliance obligation under the ETS and thus have less incentive and capacity to develop carbon trading expertise. Consequently, they were concerned that the thermal generators would be able to offer better carbonrelated skills and services and potentially take away some of their customers, thus reducing their market competitiveness (6.4.5).

The emerging market for energy efficiency products due to increasing climate change concerns and the GCCPs provided another opportunity for the generators to enhance their revenue and competitiveness. In response, most generators ( $\mathrm{A}, \mathrm{B}, \mathrm{C}$, and $\mathrm{E})$ invested substantial resources to develop and deliver energy efficiency products, particularly those based on smart metering technology (e.g. 6.2.5; 6.4.4; 6.6.5). By doing so, customer energy efficiency products become increasingly important in the generators' retail strategy.

In summary, this section has discussed the economic volatilities perceived by the generators in relation to the GCCPs and their impacts on the environmental strategies undertaken by these generators. Supporting P1, results suggest that policy and regulatory change and changing competition had implications for generators' competitiveness, while changing fuel economics and carbon market fluctuations affected their organisational (carbon and production) costs and hence, competitiveness. In turn, these external volatilities-related impacts predominantly drove changes in generation (investment), carbon credit and retail strategies. These results further provide support for $\mathrm{P} 3$, in that the impacts of these external drivers led to change in environmental strategies. The next section presents results related to the institutional pressures associated with the GCCPs.

\subsubsection{Institutional pressures}

The institutional pressures perceived by generators and their impacts on environmental strategies are presented in Table 7-4. These pressures can be classified into three categories that are broadly consistent with the institutional drivers proposed in the 
Research Framework (Figure 4-2): societal pressures; stakeholder pressures; and field and peer pressures. Additionally, a new category of institutional pressures is identified, namely: regulatory and government pressures.

\section{Table 7-4: External institutional pressures and their impacts on environmental strategies (P1, P3)}

\begin{tabular}{|c|c|c|c|}
\hline \multicolumn{2}{|c|}{ Institutional volatilities and pressures } & $\begin{array}{l}\text { Apply to } \\
\text { generator }\end{array}$ & $\begin{array}{l}\text { Influence on } \\
\text { strategy }\end{array}$ \\
\hline \multirow{2}{*}{$\begin{array}{l}\text { Regulatory } \\
\text { and } \\
\text { government } \\
\text { pressures }\end{array}$} & $\begin{array}{l}\text { RMA/ Government pressures for energy } \\
\text { efficiency and emissions reductions }\end{array}$ & All & $\begin{array}{l}\text { EP, CEE, } \\
\text { EM, ED }\end{array}$ \\
\hline & $\begin{array}{l}\text { Government preference for renewable } \\
\text { investment }\end{array}$ & All & GI, EM \\
\hline \multirow{3}{*}{$\begin{array}{l}\text { Societal } \\
\text { pressures }\end{array}$} & Associated with generation emissions & $\mathrm{A}, \mathrm{B}, \mathrm{E}$ & GI, ED \\
\hline & Associated with non-generation emissions & $\mathrm{A}, \mathrm{B}, \mathrm{C}, \mathrm{E}$ & IEE, CN, ED \\
\hline & Negative perception of windfall gains (ETS) & $\mathrm{C}$ & ED \\
\hline \multirow{3}{*}{$\begin{array}{l}\text { Stakeholder } \\
\text { pressures }\end{array}$} & Shareholder pressures & All & GI, IR \\
\hline & Greening consumer expectations & $\mathrm{A}, \mathrm{C}, \mathrm{E}$ & $\mathrm{CN}, \mathrm{EP}, \mathrm{RS}$ \\
\hline & Employee pressures & $\mathrm{A}, \mathrm{C}, \mathrm{E}$ & CN, IEE \\
\hline \multirow{3}{*}{$\begin{array}{l}\text { Field and } \\
\text { peer } \\
\text { pressures }\end{array}$} & Pressures from other generators & $\mathrm{A}, \mathrm{B}, \mathrm{E}$ & RS \\
\hline & Sustainability industry groups & $\mathrm{A}, \mathrm{C}, \mathrm{E}$ & $\mathrm{EP}, \mathrm{CN}$ \\
\hline & External consultants & All & $\begin{array}{l}\text { IEE, ED, } \\
\text { CN, EM }\end{array}$ \\
\hline
\end{tabular}

\section{Regulatory and government pressures}

The RMA as a form of environmental regulation had significant implications for the operations and development activities of all generators. Environmental compliance was a key component of all their environmental policies across all time periods. Interview data further indicate that governmental pressures for energy efficiency in the earlier periods (2000- 2005) were seen by four generators (A, B, C, D) as negative since customer energy efficiency initiatives resulted in short-term revenue decreases. Nonetheless, these generators adopted customer energy efficiency strategies primarily to comply with these government pressures. Furthermore, government pressure to reduce emissions was one of the key drivers for generators to establish emissions management and emissions-related disclosure strategies, since doing so ensured that the generators were perceived legitimate by the Government, thus gaining them governmental legitimacy.

Although not identified in the Research Framework, the interviewees argued that the Labour Government's preference for renewable generation investments was evident through GCCPs such as the PRE scheme, the NZES and the ban on thermal investment. In response to this preference, all generators announced they planned to increase renewable investments as the key strategy to manage and reduce generation-related emissions $(6.2 .3 ; 6.6 .3)$. However, once the thermal ban was removed by the National 
Government in late 2008, the institutional pressures for renewable investments reduced to some extent. However, it is not clear from the data whether such reduction in pressure led to generators putting less focus on renewables investment.

\section{Societal pressures}

The three generators with thermal generation (A, B and E) were exposed to increasing public pressures related to their generation emissions. Thermal-based Firms A and B gained a negative reputation due to the high carbon emission levels from their generation activities. This negative reputation contributed to these two generators' decisions to focus on renewable generation development, from 2007 for Firm A (6.2.4) and from 2009 for Firm B (6.3.5). Additionally, documentary data suggests that Firm E, a renewable generator with some thermal assets, was publicly criticised for its proposed thermal development which had the potential to increase total emissions significantly. In response to strong public criticism, Firm E cancelled its planned thermal project (6.6.3). All three generators (A, B, E) also increased the level of disclosure relating to emissions and emissions reduction initiatives so as to manage their social legitimacy in face of these adverse societal perceptions.

Furthermore, four generators (A, B, C, E) perceived societal pressures for nongeneration emission reduction. The generators considered that managing non-generation emissions was critical in maintaining their social legitimacy. This motivated them to undertake internal energy efficiency initiatives and achieve carbon neutrality for their non-generation activities in some years (e.g. 6.2.3; 6.42; 6.6.2). These generators also increased the scope and quality of their emissions-related disclosure to demonstrate their environmental responsibility to the wider public.

Firm C (renewable, state-owned) was also exposed to negative public perception related to its potential windfall gains under an ETS (6.4.4). This gain would come from having no carbon cost and a high electricity generation volume, allowing the generator to enhance its income significantly when wholesale prices increased. To mitigate these public perceptions, Firm $\mathrm{C}$ undertook extensive disclosure to highlight that such windfall gains originated from its proactive preparation for the GCCPs and climate change leadership in the earlier periods.

\section{Stakeholder pressures}

All generators were exposed to different shareholder pressures related to their financial performance. Privately-owned generators (A and D) operate in capital markets which require a higher rate of return from generation investments (e.g. 6.2.3). In contrast, from 
October 2008, state-owned Firms B, C and E were exposed to direct pressures from the Government, as their shareholder, to increase their financial performance (e.g. 6.3.5; 6.4.5). This resulted in these generators restructuring internally in order to improve internal efficiency and thus, maintain their legitimacy to the Government.

Furthermore, three generators (A, C, and E) perceived increasingly greening consumer expectations and employee pressures. These were considered the key drivers to their pursuit of a carbon neutrality programme in Firms A and C, since doing so was believed to increase the organisation's reputation and help attract and retain customers and talented staff $(6.2 .4 ; 6.4 .3)$. Firms $\mathrm{C}$ and $\mathrm{E}$ adopted sustainability policies and undertook green-focused retail campaigns to respond to greening consumer expectations and reinforce their green brand. Additionally, environmentally-aware employees motivated all three generators to undertake extensive internal energy efficiency initiatives (e.g. $6.4 .3 ; 6.6 .2)$.

\section{Field and peer pressures}

Field and peer pressures were significant triggers for change in generators' environmental strategies. Competitive pressures resulting from the green branding campaigns undertaken by the two renewable generators ( $C$ and $D)$ led to changes in retail strategies of other generators (A, B and E). For example, in their retail advertisement, these latter generators started to publicise their initiatives to encourage customer energy efficiency and promote internal energy efficiency. Additionally, membership in sustainability industry groups such as the SBN and NZBCSD brought about pressures on Firms A, C, E to formulate a sustainability policy and pursue a carbon neutrality programme $(6.2 .3 ; 6.4 .1 ; 6.6 .1)$. However, these pressures were not particularly strong or consistent across the different generators and different time periods.

Conversely, the impacts exerted by external consultants were evident in all generators. Landcare Research, the primary verifier and certifier of the CarboNZero programme, was an active agent in promoting particular internal energy efficiency, emissions measurement and disclosure practices and carbon neutrality strategies among the generators. Some generators also relied on advice from accounting firms to assess their potential ETS liabilities and exposure, and to formulate appropriate emissions management strategies. However, as all generators have now invested significantly in developing in-house skills and competencies to prepare for an ETS and voluntary emissions-related initiatives, the importance of external consultants is decreasing (e.g. 6.4.5). 
In summary, this section has discussed the GCCP-related external institutional pressures and identified how they affect the generators' environmental strategies. In addition to the institutional drivers suggested in the Research Framework, the results suggest a new category, namely regulatory and government pressures. In relation to $\mathrm{P} 1$, the institutional pressures primarily had implications for the generators' legitimacy, either to the Government, society, or particular stakeholder groups. However, pressures from other generators affected organisational competitiveness. To respond to these pressures, the generators employed predominantly carbon neutrality and emissions-related disclosure strategies. Nonetheless, the institutional pressures presented in this section and the economic volatilities presented in Section 7.3.1 did not influence all five generators equally. The next section analyses differences between the generators in terms of the total impacts exerted by GCCP-related external volatilities and pressures over time.

\subsubsection{Degree of total external volatility and pressure exposure}

The drivers identified in Tables 7.3 and 7.4 are combined to provide a comprehensive picture of the total external volatility and pressure exposure of the five generators to the GCCPs. Consistent with the scoring system outlined in Section 5.7.2.2, the impact of each volatility or pressure on each generator within a time period was assigned a value from 0 to 3, where 0 is not relevant/applicable, 1 is low/operational impact, 2 is medium impact, and 3 is high or strategic impact. The impacts were then summed across all the external volatilities and pressures in order to obtain the degree of total external exposure of a generator to the GCCPs during a particular time period. This summation was undertaken for all five generators across the five periods from 2000 to 2009 . The results are illustrated in Figure 7-1. 


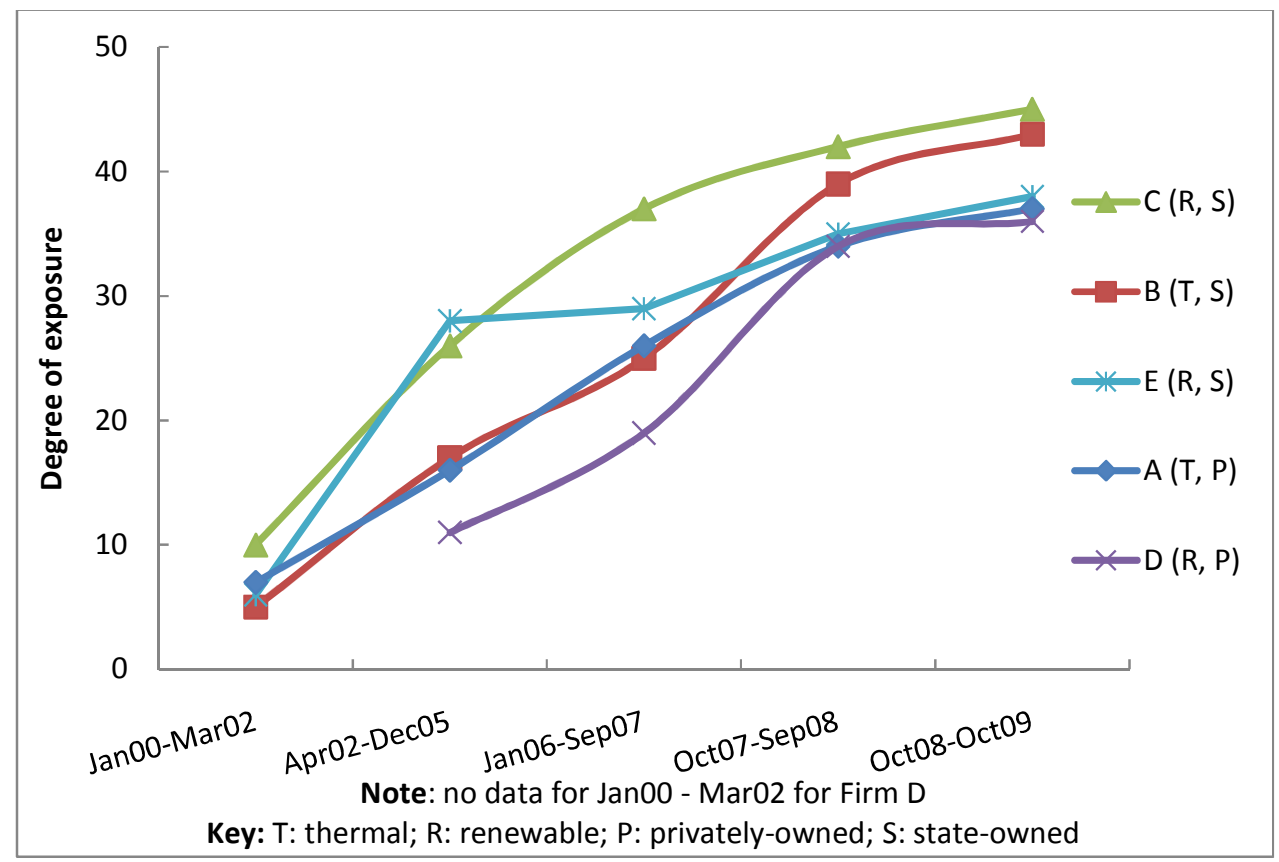

Figure 7-1 indicates that over the investigated period, the degree of exposure of the generators to the external volatilities and pressures associated with the GCCPs consistently increased. However, there are differences in the degree of exposure across the generators within each time period. From January 2000 to March 2002 when there were no GCCPs, all the generators perceived a similar and low degree of external exposure. During 2002-2006, renewable-based Firms C and E were the first generators to recognise the opportunities associated with the planned carbon tax and the PRE scheme $(6.4 .2 ; 6.6 .2)$, resulting in higher external exposure than other three generators. Due to more extensive diagnosis of GCCP-related impacts during 2006-2007, Firm C recognised an increase in the external exposure while Firm E's perceived external exposure remained unchanged. Thermal-based Firms A and B had similar degree of external exposure from 2000 to 2007, because the GCCPs were not a strategic concern in either generators $(6.2 .1$ to $6.2 .3 ; 6.3 .1$ to 6.3 .3$)$. Due to its small size (especially in terms of wholesale market share), Firm D's top management perceived little pressure or volatility associated with the GCCPs from 2002 to 2007 (6.6.4), resulting in the lowest level of exposure among the five generators.

Since October 2007, with the proposal for the Original ETS, there was a sharp increase in the external volatility and pressure exposure perceived by Firm B (thermal, stateowned) and Firm D (renewable, privately-owned). With the proposal of an ETS in 2007, Firm B recognised that its high carbon emission profile would not only cause significant carbon costs, but also expose the generator to a high level of multiple ETS-related 
economic and institutional pressures (6.3.4). In contrast, the significant increase in Firm D's external exposure is attributed to the strategic diagnosis of ETS-related impacts undertaken in 2007 which led Firm D to identify the many external market opportunities it could capture based on its renewable portfolio and expertise (6.5.3).

From October 2007 to October 2009, Firms C and B had the highest degree of external exposure while the external exposure of Firms A, D and E converged. Firm C's high GCCP exposure primarily relates to the positive impacts associated with its renewable asset advantages, which led to the decision to respond to the GCCP-related volatilities and pressures proactively and earlier than most other generators (6.3.2). Conversely, Firm B's GCCP exposure was dominated by the potential negative impacts related to its generation emissions profile (6.3.5). In contrast, while being a thermal generator historically, Firm A did not recognise a high degree of external exposure due to its efforts in the previous period to commit to a large-scale renewable investment programme and heavily publicise its commitment (6.2.4). This mitigated some of the thermal-related negative impacts it would otherwise have been exposed to. Firms D and E, although also being renewable generators, perceived a lower degree of external exposure than Firm C, mostly due to their lower wholesale market shares (Table 7-1). Further, the evaluation of GCCP-related volatilities and pressures undertaken by Firms $\mathrm{D}$ and $\mathrm{E}$ was potentially not as comprehensive and broad in scope as that of Firm C, resulting in fewer positive impacts being recognised. Figure 7-1 also indicates that the three state-owned generators $(\mathrm{C}, \mathrm{B}$ and $\mathrm{E})$ had a higher degree of external exposure than privately-owned A and D (2007-2009). This could potentially be due to a higher level of social responsibilities being perceived by the state-owned generators because of the nature of their ownership structure.

\subsubsection{Discussion: external volatilities and pressures}

Overall, the analysis reveals that the five generators were exposed to four broad categories of external economic volatilities and pressures, including policy and regulatory change, changing fuel economics, carbon market fluctuations, and changing competition. Additionally, the five generators perceived four broad categories of institutional volatilities and pressures associated with the GCCPs, including regulatory and government pressures, increased societal pressures, stakeholder pressures, and field and peer pressures. Therefore, empirical support for the six categories of economic and institutional volatilities and pressures suggested in the Research Framework was provided and evidence for two additional groups: changing competition; regulatory and government pressures, were found. Further, the results indicate that some volatilities 
and pressures were perceived by all five generators, while the others were recognised by some generators. Generally, these results suggest that the five generators are exposed to relatively homogenous GCCP-related external economic and institutional environments.

External economic volatilities were found to have implications impact on generators' competitiveness, and production and carbon costs, while the institutional pressures primarily affected the generators' social legitimacy. These results provide empirical support for P1, that GCCP-related external drivers impact on generators' competitiveness, costs, and social legitimacy. Consistent with P3, these impacts were found to drive the different environmental strategies adopted by the generators. Of the different strategies, generation (investment), carbon credit and retail strategies were the most influenced by GCCP-related external economic volatilities. This is in response to the primary impacts of such volatilities on generators' competitiveness and costs. In contrast, due to their impacts on generators' legitimacy, institutional drivers led the generators to primarily utilise carbon neutrality and emissions-related disclosure strategies in response.

Further, longitudinal analysis indicates that all generators experienced an increase in the degree of total external volatility and pressure exposure from 2000 to 2009. However, the degree of exposure varied among the generators within each time period. Two renewable state-owned generators recognised the highest exposure in the earlier periods while one thermal and one renewable generator ranked the highest in the later periods. Among these latter two, the exposure of the renewable generator was primarily associated with the advantages related to its renewable asset base, while the exposure of the thermal generator was driven by negative impacts associated with its generation emission profile. Results also indicate a higher degree of external exposure in the three state-owned generators than the two privately-owned towards the end of 2009, possibly because of their higher societal responsibilities from being state-owned.

The variations between the generators in the degree of total external exposure suggest that this exposure is not driven solely by the homogenous external environments. The differing internal characteristics and capabilities such as emissions profile and ownership structure may also have an impact. The next section presents the results regarding internal characteristics and capabilities and examines how they influenced generators and their environmental strategies. 


\subsection{Internal GCCP-related drivers}

This section addresses P2 and P3 by presenting findings related to the impacts of internal drivers on the generators and their associated environmental strategies. Based on the interpretation of Chapter 6-based evidence, these internal drivers can be classified into two groups. The first group (7.4.1 and 7.4.2) is comprised of the internal characteristics that increase the negative impacts of the GCCPs on the generators. The second group (7.4.3 and 7.44) relates to the internal capabilities that result in positive impacts by the GCCPs on generators and enhance the generators' ability to undertake environmental strategies.

\subsubsection{Internal characteristics}

Table 7-5 lists the internal characteristics that result in negative impacts from the GCCPs on the generators and identifies the environmental strategies that were driven by these characteristics. All characteristics can be broadly grouped into the categories proposed by the Research Framework, namely: emissions profile, transactional characteristics, and organisational size. However, the third category is renamed as organisational size and growth in order to include an additional internal driver, i.e. organisational growth. A new category is also found and named technological and financial constraints, to reflect their GCCP-related negative impacts on the generators and to distinguish from technological capabilities which have positive organisational impacts (7.4.3). Each category of internal characteristic is discussed next.

Table 7-5: Internal characteristics and their impacts on environmental strategies (P2, P3)

\begin{tabular}{|c|c|c|c|}
\hline \multicolumn{2}{|c|}{ Internal characteristic } & $\begin{array}{l}\text { Apply in } \\
\text { firms }\end{array}$ & $\begin{array}{l}\text { Influence } \\
\text { strategy }\end{array}$ \\
\hline \multirow{3}{*}{ Emissions profile } & Generation emissions & $\mathrm{A}, \mathrm{B}, \mathrm{E}$ & $\begin{array}{l}\text { EM, GI, } \\
\text { CC, ED, PS }\end{array}$ \\
\hline & \multirow{2}{*}{ Non-generation emissions } & All & IEE, ED \\
\hline & & A, C, D & $\mathrm{CN}$ \\
\hline \multirow{4}{*}{$\begin{array}{l}\text { Transactional } \\
\text { characteristics }\end{array}$} & Generation emissions measurement and monitoring & $\mathrm{A}, \mathrm{B}, \mathrm{E}$ & EM \\
\hline & $\begin{array}{l}\text { Non-generation emissions measurement and } \\
\text { monitoring }\end{array}$ & All & IEE, ED \\
\hline & Carbon trading transactions & $\mathrm{A}, \mathrm{B}, \mathrm{E}$ & $\mathrm{CC}$ \\
\hline & Carbon-related cross functional coordination & All & $\begin{array}{l}\mathrm{EM}, \mathrm{CC} \\
\mathrm{IEE}, \mathrm{CN}\end{array}$ \\
\hline \multirow{2}{*}{$\begin{array}{l}\text { Organisational } \\
\text { size and growth }\end{array}$} & Organisational size & $\mathrm{D}$ & IEE, ED \\
\hline & Organisational growth & All & $\mathrm{IEE}, \mathrm{CN}$ \\
\hline \multirow{2}{*}{$\begin{array}{l}\text { Technological and } \\
\text { financial } \\
\text { constraints }\end{array}$} & $\begin{array}{l}\text { Technological difficulty in reducing generation } \\
\text { emissions }\end{array}$ & $\mathrm{A}, \mathrm{B}, \mathrm{E}$ & EM, GI \\
\hline & Financial constraints & $\mathrm{B}, \mathrm{C}, \mathrm{E}$ & IEE, CC \\
\hline
\end{tabular}




\section{Emissions profile}

As expected, generation emissions were a major concern for all the generators with thermal assets (A, B and E) (Table 7-1). The level of generation emissions determines the degree of emissions liabilities and costs under the ETS or a carbon tax. Generation emissions were found to drive emissions management strategy within Firms A, B and E. Firm B initially focused on managing the fuel use within its thermal plants to reduce generation emissions (6.3.2) while Firms $\mathrm{A}$ and $\mathrm{E}$ increased renewable investments to replace its existing thermal plants $(6.2 .3 ; 6.6 .4)$. However, due to strong societal pressures, Firm B decided to shift to a renewable-focused generation strategy from October 2008 (6.3.5). Furthermore, these generators set up internal carbon trading functions to ensure effective management of carbon credit purchases and sales and hence, the management of their ETS compliance costs $(6.2 .5 ; 6.3 .4 ; 6.6 .5)$. These three generators (A, B and E) also adopted appropriate emissions-related disclosure to demonstrate their environmental responsibility and mitigate adverse public reputation related to their emission profile (e.g. 6.2.5; 6.3.3). Having a high emission profile motivated these generators to participate in the policy processes with an aim to reduce their potential obligations under the GCCPs (e.g. 6.6.4).

A different story is gained in relation to non-generation emissions. While nongeneration emissions do not contribute to a generator's carbon tax/ ETS liabilities, they were perceived by all generators to contribute to the public perception of organisational climate change responsibility and hence were related to societal pressures for emissions reductions. Consequently, the five generators undertook various internal energy efficiency initiatives to reduce their non-generation emissions. As well, they disclosed these initiatives publicly to maintain social legitimacy (e.g. 6.4.3; 6.6.4). Furthermore, due to their high levels of non-generation emissions (Table 7-1), Firms A, C and D incurred high costs because of the need to pay for carbon offsets when pursuing their carbon neutrality certification.

\section{Transactional characteristics}

The three generators with thermal generation (A, B and E) experienced difficulties in measuring emissions. These generators need to monitor and report their emissions for compliance purposes under an ETS. However, the rate of fossil fuel use for generation varies not only across seasons or months, but also across hours and days and generation plants. This results in varying levels of generation-related carbon emissions which made accurate measurement and monitoring difficult (representing high transactional costs), which in turn affects the emissions management strategy of these generators. 
Non-generation emissions, for all the generators, were also hard to measure and monitor, because such emissions are associated with a wide range of operational activities and functions that occur throughout the organisation. This results in high transactional complexity which in turn hinders the generators' ability to implement their internal energy efficiency strategies effectively (high transactional costs). Interview data indicate this complexity contributed to some generators (A, B, D) not being able to disclose their non-generation emissions on at an organisationally-wide or detailed level $(6.3 .4 ; 6.5 .2)$.

Further, the three generators with ETS compliance obligations (A, B, E) perceived a high level of volatility associated with carbon trading transactions. These generators were concerned about their lack of expertise in trading carbon credits and evaluating the risks inherent in carbon contracts. Carbon trading thus involves both specialised knowledge (high asset specificity) and a high level of market volatility (transactional uncertainty), which led to high transactional costs for generators participating in carbon markets for compliance or trading purposes (e.g. 6.6.5). To manage this lack of expertise, these generators begun to develop their internal carbon trading capability, for example, through establishing a carbon trading function (6.4.3).

All the generators experienced difficulties with carbon-related cross-functional coordination. To meet their compliance obligations under the ETS, Firms A, B and E need input and coordination from multiple organisational functions. Specifically, coordination is required between the environmental management function which monitors and manages generation emissions, the trading function which buys and sells carbon credits, and the accounting function which records the carbon credits inventory and reports the organisational financial exposure given the current emission levels and carbon prices (e.g. 6.2.5). These generators (A, B and E) also perceived a need for cross-functional coordination to achieve organisation-wide measurement, monitoring and the reduction of non-generation emissions (internal energy efficiency strategy). For the renewable generators $C$ and $D$, it is essential for the accounting function to keep a record of existing carbon credits (i.e. gained from PRE scheme), for the trading function to sell these credits, for each operational or non-operational function to monitor and report their non-generation emissions, and for the sustainability function to offset total organisational emissions to achieve carbon neutrality certification (6.4.4). Consequently, such complex internal coordination results in high transactional costs when implementing the various environmental strategies pursued by the generators. 


\section{Organisational size and growth}

Organisational size also influences a generator' exposure to the GCCP-related external volatilities and pressures. Firm D is smaller than other generators (Table 7-1). Because of its smaller size (and being 100\% renewable), Firm D is not as "visible" as other generators in the public arena and thus, was exposed to a lower level of societal and governmental pressures for emissions reductions. This explains why Firm D's internal energy efficiency initiatives and emissions-related disclosure were of a more limited scope and received less managerial attention, than in other generators (6.5.4).

Additionally, all generators experienced organisational growth (to varying degrees) over time, which resulted in increases in non-generation emissions. For example, an increase in customer numbers required additional staff travel to service and maintain customers; or the construction or commissioning of a new generation plant led to recruitment of new employees, resulting in increased use of electricity and paper use, and waste. Such organisational growth led to increasing operating costs associated with the additional resource requirements (e.g. 6.4.2). Furthermore, interview data indicates that the resultant increase in total non-generation emissions put pressure on the internal energy efficiency strategy of all generators and required higher offset payments for those emissions in order to gain carbon neutrality certification (Firms A, C and D).

\section{Technological and financial constraints}

The technological constraints inherent in thermal generation assets represented a significant pressure for three generators (A, B and E). Firms A and E's plants either had a low emissions level (gas-fired) or had a fixed fuel use, resulting in significant difficulties in reducing generation emissions. In contrast, the technology in Firm B's thermal plants allowed it to switch from coal to gas to reduce the level of carbon emissions. However, Firm B was constrained by growing electricity demand and the need to operate all of its thermal plants to meet supply shortages caused by low hydro levels in dry years (6.3.5). This demand/supply constraint led Firm B to increase its reliance on coal and thus substantially curtailed its ability to switch between fuels to reduce generation emissions. Overall, these technological constraints represent high asset specificity and resulted in high transactional costs, which drove all three generators to change their emissions management strategy and generation strategy towards a preference for renewable investments.

This study did not find any evidence that the generators experienced any financial constraints when implementing their environmental strategies in the earlier periods. 
This could be explained by the fact that most generators are large firms (Table 7-1) and thus have sufficient financial resources to support organisational changes in response to the GCCPs. However, from October 2008 to October 2009, under performance pressures from Government, the state-owned generators (B, C and E) experienced a substantial reduction in spending discretion and thus were forced to decrease the scope of their internal energy efficiency initiatives in an attempt to cut costs $(6.3 .5 ; 6.4 .5$; 6.6.5). Government-imposed financial constraints also resulted in Firm C moving away from developing a carbon trading business to focus on its core operations (electricity generation and retailing) (6.4.5).

To summarise, the above analysis indicates both similarities and differences between the generators' internal characteristics. Some internal characteristics were perceived by all five generators as driving their GCCP exposure and influencing their environmental strategies. Other internal characteristics were experienced by some or only one generator. Supporting P2, results indicate that the generators' emission profile and organisational size had implications for organisational carbon and operating costs and legitimacy, while transactional characteristics, organisational growth, and technological and financial constraints influenced transactional and operating costs. These internal characteristic-driven impacts predominantly affected emissions management, internal energy efficiency, carbon credit and emissions-related disclosure strategies (P3).

Furthermore, these results indicate that internal characteristics including emissions profile (generation and non-generation related) and organisational size increase the generators' exposure to external economic and institutional drivers, such as carbon market fluctuations and societal and Governmental pressures and hence, affect their social legitimacy. In this respect, internal characteristics moderated generators' exposure to external drivers by increasing the negative impacts of such drivers on the organisation. This is a new insight not available from the prior literature.

\subsubsection{Total impact exerted by internal characteristics}

Following the scoring system outlined in Chapter 5 (5.7.2.2), the degree of impact exerted by the internal characteristics (identified in 7.4.1) on the generators' exposure to the GCCPs was assigned a value from 0 to 3: 0 : not relevant/applicable, 1: low/operational impact, 2: medium impact, 3: high/strategic impact. In a data matrix display, the scores were then totaled to obtain the degree of total impact exerted by internal characteristics, on each generator within each time period. Figure 7-2 shows this impact across the five generators from 2000 to 2009. 


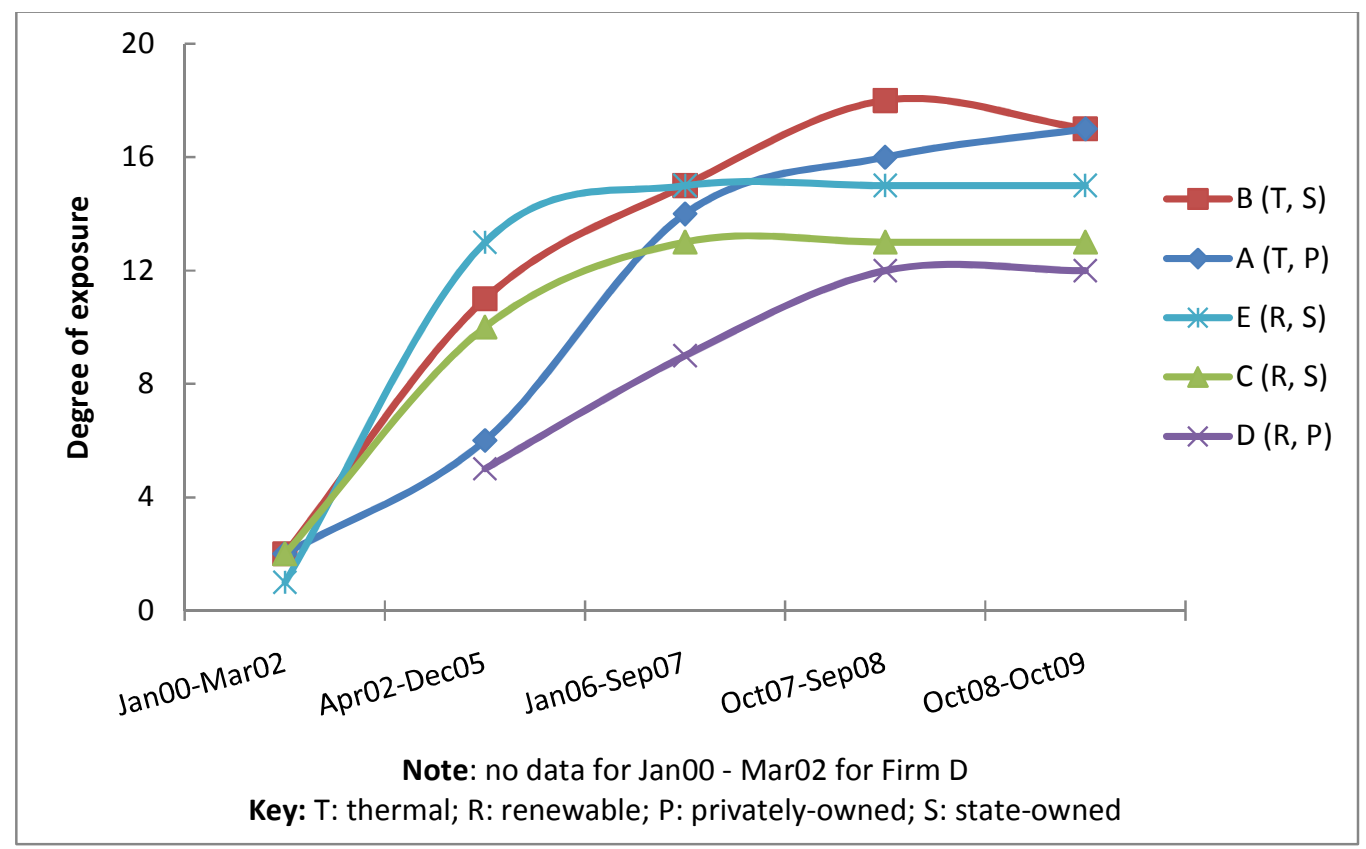

In 2000, most of the generators had a similar and low level of impact from the internal characteristics. From April 2002 to December 2005, the state-owned generators (B, C, and E) experienced relatively similar increases which are higher than privately-owned A and D. This is possibly due to a higher need of the state-owned generators to maintain legitimate to the Government and society through the undertaking of climate change initiatives, a process that led them to recognise a stronger influence by internal characteristics on organisational costs and legitimacy. Firm E experienced the highest level of impact due to the complexity associated with the early initiatives to improve its environmental management system and its focus on internal energy efficiency to achieve the external Enviro-mark certification (6.6.2). Firm B had the second highest level, which is potentially attributable to the emissions management and internal energy efficiency initiatives it undertook to demonstrate its climate change responsibility (6.3.2). Firm $\mathrm{C}$ conducted an extensive internal energy efficiency programme, to show its leadership in climate change issues (6.4.2). In contrast, consistent with their private ownership structure, Firms A and D were not exposed to strong institutional pressures during the early period. Consequently, they did not undertake many climate change initiatives and thus, perceived a lower level of impact associated with internal characteristics during the implementation of these initiatives.

From January 2006, the impact exerted by internal characteristics on Firms C and E stabilised, while that of the thermal generators (A and B) and renewable-based Firm D continued to increase. By October 2009, due to their higher emission profiles and 
resultant carbon costs, Firm A and B experienced the highest degree of impact. This is because both generators perceived the technological difficulties associated with reducing generation emissions and the complex internal coordination required to enable emissions monitoring, and carbon credit purchases to meet ETS compliance requirements. The other three generators had either no ETS compliance obligations (Firms C and D), or a low level of emissions liabilities (Firm E). Therefore they experienced a lower degree of impact associated with the internal characteristics.

The generators differed from each other not only in terms of internal characteristics' impact but also in relation to impact of internal capabilities. The next section discusses the key internal capabilities possessed by the different generators and their impacts on the generators (P2) and implications for their environmental strategies (P3).

\subsubsection{Internal capabilities}

The evidence presented in Chapter 6 suggests that various internal capabilities were perceived by the managers of different generators as being critical in the management of the organisation's exposure to GCCP-related volatilities and pressures. In particular, possessing these capabilities increased the advantages that the generators could gain under the GCCPs. These capabilities also influenced the generators' ability to pursue and implement environmental strategies. They can be classified into the broad categories suggested in the Research Framework: technological capabilities, managerial factors, prior experience and organisational learning, and organisational culture. Data analysis also indicates a new category: ownership-related capabilities. Table 7-6 lists these categories of internal capabilities, specifies which generators they applied to, and identifies the environmental strategies they influenced. Each category of internal capability is discussed below. 


\begin{tabular}{|c|c|c|c|}
\hline \multicolumn{2}{|c|}{ Internal capabilities } & $\begin{array}{l}\text { Apply in } \\
\text { firms }\end{array}$ & $\begin{array}{l}\text { Influence } \\
\text { strategy }\end{array}$ \\
\hline \multirow{7}{*}{$\begin{array}{l}\text { Technological } \\
\text { capabilities }\end{array}$} & Possession of carbon credits & $\mathrm{B}, \mathrm{C}, \mathrm{D}, \mathrm{E}$ & $\mathrm{CC}$ \\
\hline & \multirow{2}{*}{ Net generation volume } & $\mathrm{A}, \mathrm{B}, \mathrm{C}, \mathrm{E}$ & $\mathrm{RS}$ \\
\hline & & $\mathrm{D}$ & GI, RS \\
\hline & Access to renewable resource sites & $\mathrm{A}, \mathrm{C}, \mathrm{D}, \mathrm{E}$ & GI \\
\hline & Availability of fuel supply & $\mathrm{B}, \mathrm{E}$ & GI \\
\hline & Economies of scale in renewable development & $\mathrm{A}, \mathrm{C}, \mathrm{D}, \mathrm{E}$ & GI \\
\hline & Inheritance assets & $\mathrm{C}, \mathrm{D}$ & RS, PS \\
\hline \multirow{5}{*}{$\begin{array}{l}\text { Managerial } \\
\text { factors }\end{array}$} & Top management support of renewables & A, C, D & GI \\
\hline & Top management commitment to sustainability & $\mathrm{C}, \mathrm{E}$ & $\mathrm{EP}, \mathrm{IEE}$ \\
\hline & Board involvement & $\mathrm{C}$ & GI \\
\hline & \multirow{2}{*}{$\begin{array}{l}\text { Management recognition of external volatilities } \\
\text { and pressures }\end{array}$} & $\mathrm{B}$ & EM, ED \\
\hline & & $\mathrm{C}, \mathrm{E}$ & $\mathrm{RS}, \mathrm{GI}, \mathrm{CEE}$ \\
\hline \multirow{3}{*}{$\begin{array}{l}\text { Prior } \\
\text { experience and } \\
\text { organisational } \\
\text { learning }\end{array}$} & Experience in emissions reduction & $\mathrm{B}$ & EP, EM \\
\hline & Renewable expertise & $\mathrm{A}, \mathrm{C}, \mathrm{D}, \mathrm{E}$ & GI, PS \\
\hline & Experience in customer energy efficiency & $\mathrm{E}$ & CEE, RS \\
\hline $\begin{array}{l}\text { Organisational } \\
\text { culture }\end{array}$ & Environmental/climate change awareness & $\mathrm{C}, \mathrm{E}$ & IEE, GI \\
\hline \multirow{2}{*}{$\begin{array}{l}\text { Ownership- } \\
\text { related } \\
\text { capabilities }\end{array}$} & Risk appetite and cost consciousness & $\mathrm{A}, \mathrm{D}$ & GI, EM, IEE, CC \\
\hline & Relationship with majority shareholder & A & $\mathrm{CC}, \mathrm{GI}$ \\
\hline
\end{tabular}

\section{Technological capabilities}

Different technological capabilities were found in the five generators. Four generators (except Firm A) participated in the Government's PRE scheme and were granted carbon credits, which allowed them to develop early carbon trading skills and gain carbon revenue (e.g. 6.3.4; 6.4.3). Firms B and E planned to use these carbon credits to offset their future ETS obligations, thus reducing their exposure to carbon market fluctuations (e.g. 6.6.5).

Net generation volume (i.e. the difference between total generation volume and retailing commitments) is a capability that enabled four generators (A, B, C and E) to benefit from a probable increase in wholesale electricity prices due to the ETS (Table 7-1). Due to their net generation volume, these generators are able to undertake retail campaigns to attract and service more customers. However, Firm D is a net buyer, and therefore a wholesale price increase is perceived as a negative impact. To reduce its exposure to wholesale market fluctuations, Firm D sought to increase its generation-retail balance by developing further generation capacity and reducing its retail customer base where possible (6.5.1). 
Further, access to resource sites, for example, a geothermal field, was considered by four generators (A, C, D, E) as critical to their ability to take advantage of GCCPrelated changing fuel economics and build additional generation capacity. Accordingly, these generators focused their generation investments in areas where they already had secure access to, for example, through a resource consent (e.g. 6.2.4). In contrast, the availability of (fossil) fuel supply was an important consideration for the generators pursuing thermal investments $(\mathrm{B}, \mathrm{E})$. These generators were involved in gas exploration activities and thus earned the entitlements to future gas supply that would ensure operational efficiency of their proposed thermal plants $(6.3 .2 ; 6.6 .2)$.

Economies of scale in renewable development helped most generators (except Firm B) to respond positively to the changing fuel economics under the GCCPs. Such economies of scale enabled these generators to pursue further renewable investments at a lower cost. For example, these generators were able to negotiate favourable deals with suppliers to reduce the costs of generation equipment, such as wind turbines (6.4.3). Furthermore, economies of scale improved operational efficiency, which in turn potentially reduces production costs (e.g. 6.4.3). Additionally, renewable-only inheritance assets were a significant advantage for Firms C and D since these assets enhanced these generators' reputation and enabled them to undertake green-branding retail strategies and enhance their market competitiveness, in a changing competitive environment. The renewable asset base also exposed these generators to less pressures from society to reduce emissions, compared to thermal-based ones. This inherited renewable asset base also provides an incentive for Firms C and D to strongly support the ETS and even recommend additional policies to maximise potential gains from their assets at the expense of thermal generators (6.4.4.; 6.5.2).

\section{Managerial factors}

Top management support for renewable development was an internal capability in three generators (Firms A, C, D). Firm A was historically a thermal generator but in recent years has focused its generation investments on renewable sources (6.2.3). In contrast, top management support had always been a key driver of Firms C and D's renewableonly generation strategy and thus their development of renewable-focused competitive advantage (e.g. 6.5.1). Furthermore, top management commitment to sustainability enabled Firm $\mathrm{C}$ and $\mathrm{E}$ to adopt a sustainability policy early on and undertake wider scope internal energy efficiency initiatives (e.g. 6.6.2). Additionally, active board involvement in the strategic planning process was considered an important driver of Firm C's decision to pursue a renewable-only generation (investment) strategy (6.4.2). 
Management recognition of external volatilities and pressures are also important drivers of environmental strategies in three generators (Firms B, C and E). Firm B's managers perceived negative impacts from GCCP-related volatilities and pressures. Consequently, its environmental strategies were aimed primarily at mitigating these impacts, i.e. reducing generation carbon emissions, and adopting environmental disclosure to respond to the societal pressures (6.3.3). In contrast, Firm C's top management interpreted external volatilities and pressures as primarily opportunities and thus more proactive strategies were pursued, i.e. green marketing and branding, and early investments in renewable assets (6.4.2). Similarly, top management in Firm E also recognised the opportunities associated with emerging energy efficiency markets early and thus undertook customer energy efficiency initiatives as part of its retail strategy (6.6.2).

\section{Prior experience and organisational learning}

Due to its experience in emissions reduction, Firm B was able to integrate emissions management as a key objective in its environmental policy (6.3.2) and reduce its generation emissions and thus carbon costs more effectively than Firms A and E. In contrast, prior experience in renewable energy provided a substantial advantage for the other four generators (A, C, D, E) since it enhanced their competitiveness in a GCCP operating environment which incentivises renewable investments. Thus, these generators were able to effectively respond to GCCP-related changing fuel economics and follow a differentiation strategy in generation investment. ${ }^{11}$ Renewable expertise also motivated these generators to politically support the ETS and the Government's preference for renewable energy. In particular, Firms $\mathrm{A}$ and $\mathrm{E}$ who have compliance obligations under the ETS lobbied the Government to reduce their potential carbonrelated compliance liabilities (for example, by suggesting the exclusion of geothermal emissions from the ETS) (6.6.4). Additionally, Firm E's early experience in customer energy efficiency enabled it to develop innovative energy efficiency services and products (6.6.3), based on which its retail competitiveness was enhanced, especially in an emerging energy efficiency market.

\section{Organisational culture}

Organisational culture was considered an important internal capability for some generators. Firm $\mathrm{C}$ had a corporate culture built around internal innovation, entrepreneurship and high environmental awareness (6.4.3). Similarly, Firm E

\footnotetext{
${ }^{11}$ Firm A, while being historically a thermal generator, invested heavily in geothermal projects and developed substantial geothermal expertise.
} 
developed a strong culture associated with environmental responsibility (6.6.2). In both generators, such environmentally-focused culture provided significant support and motivation for the pursuit of extensive internal energy efficiency initiatives and largescale renewable investments. A supportive corporate culture also increased the effectiveness with which Firms $\mathrm{C}$ and E deployed other internal capabilities, such as prior experience in renewable energy and energy efficiency, in implementing their environmental strategies. Additionally, the environmentally-focused organisational culture was well publicised (e.g. 6.6.2) and thus contributed to an enhancement of these generators' social legitimacy.

\section{Ownership-related capabilities}

In addition to the drivers proposed in the Research Framework, this study found that the generators' ownership structure had implications for their environmental strategies. The privately-owned generators ( $\mathrm{A}$ and $\mathrm{D}$ ) had a different risk appetite from that of stateowned ones, as the privately-owned generators require a higher rate of return on generation investments $(6.2 .3 ; 6.5 .1)$. Furthermore, there was higher cost consciousness among their managers and employees because of strong performance pressures associated with the capital markets. As a result, their environmental strategies (emissions management, internal energy efficiency and carbon credit strategies) featured strong cost control components. Additionally, the close relationship with a majority shareholder enabled Firm A to access this organisation's expertise and resources in carbon trading and generation development (6.2.5). Such access allowed Firm A to pursue lower-cost carbon credit and generation strategies, as well as facilitate organisational learning and adaptation to changes in external carbon trading and investment environments (i.e. reducing transactional cost). It also reduced Firm A's exposure to carbon market fluctuations when sourcing the credits required to meet its ETS compliance obligations.

Overall, the results from analysing generators' internal capabilities provide support for the drivers suggested in the Research Framework. However, a new category is found, ownership-related capabilities. The four generators with large renewable investment programmes (A, C, D, and E) share a number of similar internal capabilities, including access to renewable resource sites, top management support or commitment, and renewable expertise. Other capabilities were only relevant to either one, two or three generators. In support of $\mathrm{P} 2$, the analysis suggests that the internal capabilities identified impact on the generators' competitiveness, costs, and legitimacy. Specifically, technological capabilities and prior experience and organisational learning improved 
generators' competitiveness or enabled them to control organisational costs. Managerial factors primarily had implications for generators' competitiveness, while organisational culture enhanced their social legitimacy. In contrast, ownership-related capabilities led to more effective cost control. In response, the generators formulated and modified environmental strategies to take advantage of their internal capabilities (P3). The most predominant GCCP-related environmental strategies affected by internal capabilities were generation investment and retail strategies.

Furthermore, the results suggest that a number of these internal capabilities moderated the generators' exposure to external drivers. For example, possession of carbon credits and a relationship with a majority shareholder enabled some generators with ETS compliance obligations to be less vulnerable to carbon market volatility. Renewablebased inheritance assets reduce some generators' exposure to societal pressures and improve their organisational reputation, while prior experience enabled them to gain a competitive advantage from changing fuel economics and the emerging energy efficiency market. Consequently, possessing internal capabilities enhanced the positive impacts of external drivers on the generators. This moderating impact of internal capabilities is a new insight that is not available from the prior literature.

\subsubsection{Degree of total internal capabilities}

Using an Excel-based matrix data display, each of the internal capabilities identified in 7.4.3 were quantified based on the degree of their availability and strength within each generator (from 0: not perceived or not available; to 3: strong capability) (5.7.2.2). The degree of total internal capabilities implies the total availability and strength of GCCPrelated internal resources possessed by a generator within a particular time period. The degree of total capabilities possessed by the five generators from 2000 to 2009 is illustrated in Figure 7-3. 


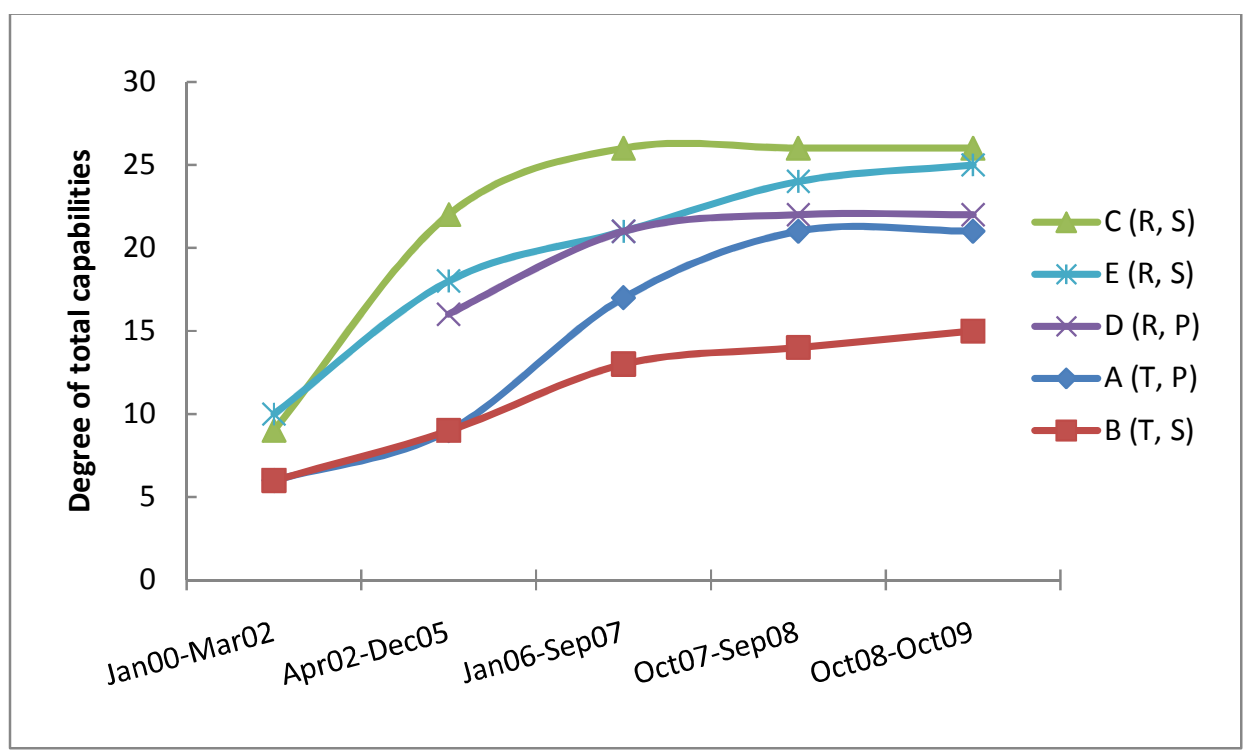

From January 2000 to September 2007, there was clear distinction in the degree of total internal capabilities between renewable generators $(\mathrm{C}, \mathrm{D}, \mathrm{E})$ and thermal ones $(\mathrm{A}, \mathrm{B})$ with the renewable generators having a higher degree of total capabilities. Among the renewable generators, Firm $\mathrm{C}$ had the highest degree of internal capabilities because it had the strongest internal capabilities including: early managerial recognition of GCCPrelated external volatilities and pressures; active board involvement in strategy planning process; renewable assets and expertise; and top management support and commitment (6.4.2). The degree of internal capabilities within Firms D and E was also higher than the thermal generators because they were developing significant renewable expertise and had top management support and commitment to renewable investment. Between January 2000 and December 2005, Firms A and B had a similar degree of internal capabilities, reflected by their net generation volume, inheritance assets, experience in emission reduction (B), and some renewable expertise (A). Since during that time, they were still highly thermal-focused, Firms A and B did not develop the same degree of renewable-based GCCP-related internal capabilities as the renewable generators.

Interestingly, from January 2006 to October 2009, the degree of total capabilities possessed by Firm A increased at a high rate, and reached almost the same level as that of Firm D. This increase is associated with the change in generation strategy of Firm A in 2007 to focus on large-scale geothermal development (6.2.4). The result was the channelling of organisational resources to develop strategic renewable-related capabilities. In this period, Firm E also experienced a large increase in its internal capabilities. This is potentially attributed to its strategic decision to adopt renewablefocused generation investment and retail strategies in order to take advantage of GCCP- 
related market opportunities $(6.6 .4 ; 6.6 .5)$. The degree of total capabilities in Firms C and D increased during Jan2006-Sep2007 to support their renewable-only generation strategy, but stabilised from October 2007 to October 2009. This is because these two generators had developed all of their available internal capabilities to a strong level during the previous periods, and thus any incremental change in the strength of each capability during 2007-2009 was not shown.

By October 2009, there was a degree of convergence between Firms A, C, D and E in the degree of their total internal capabilities. These capabilities primarily enabled these four generators to enhance their competitiveness and pursue renewable-focused environmental strategies. In contrast, Firm B ranked the lowest because it was still strongly thermal-based throughout the period and thus, had few renewable-related capabilities to build on. However, it did develop other capabilities, including increasing generation volume, obtaining PRE carbon credits, possessing a secure fossil fuel supply, and gaining experience in emissions reduction. These capabilities primarily enabled Firm B to manage the carbon and production costs associated with the implementation of its environmental strategies.

\subsubsection{Discussion: internal characteristics and capabilities}

The above sections have analysed the impacts of internal characteristics and internal capabilities on the generators and their environmental strategies. The results provide broad support for all the internal drivers identified in the Research Framework. Additionally, the results suggest a number of new drivers that have not been examined in the prior literature or the Research Framework. They are organisational growth and financial and technological constraints (as internal characteristics), and ownershiprelated capabilities (as internal capabilities).

The results suggest that the generators' internal characteristics impact on their costs and legitimacy, but not competitiveness. All internal characteristics affect generators' organisational costs, except for emissions profile and organisational size, both of which had implications for organisational costs and social legitimacy. In contrast, internal capabilities enhance the generators' competitiveness and legitimacy as well as enabling better cost control. Consequently, these results suggest that the internal drivers affect the generators' competitiveness, costs, and legitimacy, thereby largely supporting P2.

Consistent with P3, the impacts exerted by internal drivers led to changes in the generators' environmental strategies. Due to their influence on organisational costs and social legitimacy, internal characteristics predominantly affected emissions 
management, internal energy efficiency, carbon credit and emissions-related disclosure strategies. In contrast, the predominant strategies affected by internal capabilities were generation investment and retail strategies, because such capabilities had a strong impact on generators' competitiveness (alongside costs and legitimacy).

In addition to the support found for propositions and the Research Framework, new insights are found. Internal characteristics and capabilities moderate the generators' exposure to the external GCCP-related drivers. Internal characteristics increase the negative impacts of the external drivers by exposing the generators to external economic and institutional drivers and hence imposing higher costs and/or threatening their legitimacy. In contrast, internal capabilities enhance the positive impacts of the external drivers on the generators by enabling them to take advantage of these external drivers to improve their competitiveness, legitimacy, or reduce costs more effectively.

Furthermore, the differences found in the set of internal characteristics and capabilities (drivers) that characterised the different generators suggest that the generators were operating in relatively heterogeneous internal environments (Tables 7-5 \& 7-6), as opposed to the relatively homogenous GCCP-related external environments (Tables 7-3 \& 7-4). The results also indicate that from 2000 to 2009 all generators perceived an increasing degree of total external exposure and impact by external volatilities and pressures, as well a growing level of total capabilities. Additionally, the thermal generators (A, B) were found to have the highest degree of GCCP-related exposure driven by their internal characteristics, while the renewable generators (C, D) experienced lower exposure. Further, a higher degree of total internal capabilities was found in generators with renewable expertise and assets $(\mathrm{A}, \mathrm{C}, \mathrm{D}$, and $\mathrm{E})$, than that in thermal-based Firm B. In response to such variations in external and internal drivers over time and across generators, the generators changed their environmental strategies differently, as reflected by the level of strategy proactiveness and changes in strategic emphasis, as discussed in the next section.

\subsection{Change in environmental strategies}

It is clear from the preceding sections that the five generators changed their environmental strategies in response to the GCCP-related external and internal drivers. To further understand the process of change in environmental strategies, this section analyses the dynamics and variations in the way strategic changes were employed and implemented across the different generators over time. Doing so also provides a further basis to examine the association between environmental strategies and the impacts of 
external and internal drivers (P3). As outlined in Section 4.4.4, this study analyses change in environmental strategies from two aspects: strategy proactiveness and strategic emphasis. Firstly, to assess how early (timing) environmental strategies were formulated and how broadly (scope) they were implemented within the organisation, the level of strategy proactiveness is investigated. Secondly, changes in strategic emphasis are examined to understand how much top management attention was assigned to environmental strategies within each generator and whether this attention changed over time. Thus, these two aspects of change in environmental strategies indicate i) how proactive the change was, and ii) how much attention management paid to such a change.

\subsubsection{Strategy proactiveness}

To facilitate the analysis of strategy proactiveness, a high level of environmental strategy proactiveness is considered to capture three characteristics:

i. the strategy was formulated to respond to GCCP-related volatilities and pressures early and ahead of the introduction of any GCCP-associated regulations,

ii. the initiatives undertaken were extensive and substantial financial provision was made for them; and,

iii. multiple organisational functions were involved in implementing these initiatives.

In contrast, a low level of proactiveness (i.e. a reactive environmental strategy) implies mere conformance to existing environmental regulations and other institutional pressures that prevail in the existing operating environments, and involves narrowscoped initiatives and few organisational functions. In a data matrix display, each of the eleven environmental strategies listed in Table 7-2 was assigned a score of 0, 1, 2 or 3, depending on the perceived level of proactiveness during each time period (5.7.2.3). The scores were then added across the eleven environmental strategies to obtain a total strategy proactiveness value for each generator in each time period. For each generator, the trend of change in this value over time was then analysed in relation to the change in the degree of total external volatility and pressure exposure, degree of total impact by internal characteristics, and degree of total capabilities (P3).

The analysis did not find any direct association between a change in total strategy proactiveness and a change in total impact of internal characteristics over time. This is unexpected, given that internal characteristics were found to affect individual 
environmental strategies (Table 7-5). Future research can further examine this relationship to confirm whether an association between the total impact of internal characteristics and strategy proactiveness exists. However, there appears to be significant similarities between change in strategy proactiveness levels and changes in degree of total external volatility and pressure exposure (Figure 7-1) and the degree of total internal capabilities (Figure 7-3). These similarities are illustrated in the graphs in Figure 7-4.

Figure 7-4: GCCP-related external exposure, internal capabilities and strategy proactiveness (2000-2009) (P3)

Firm A

(thermal, privately-owned)

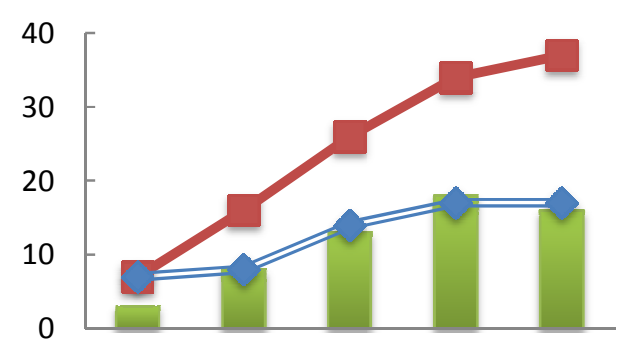

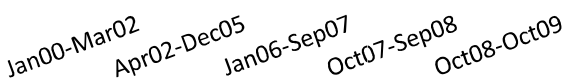

Firm B

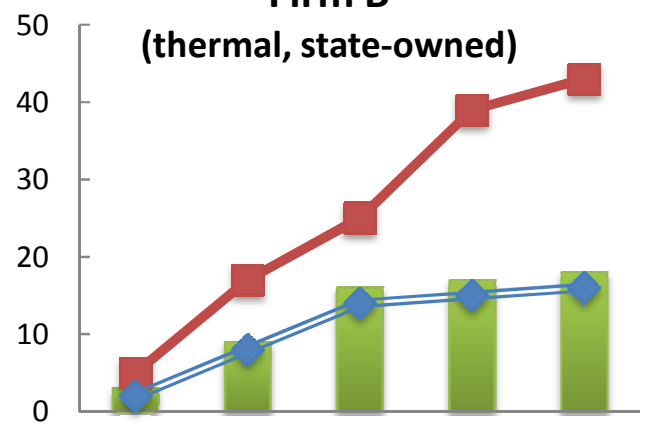

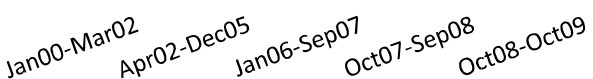

Firm C

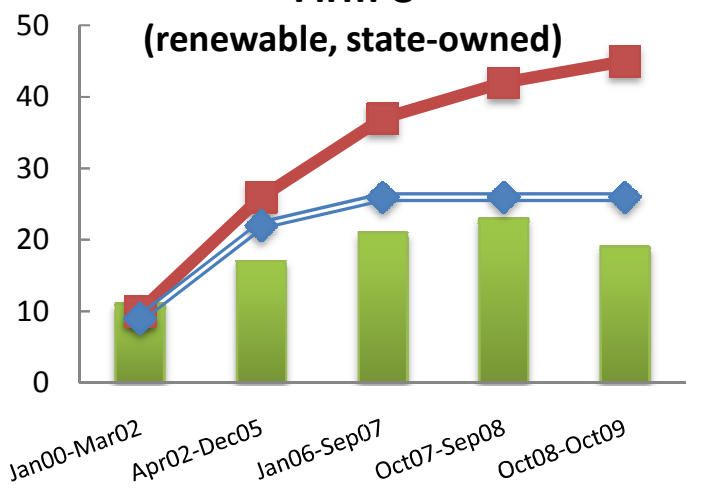

Firm D

(renewable, privatelyowned)

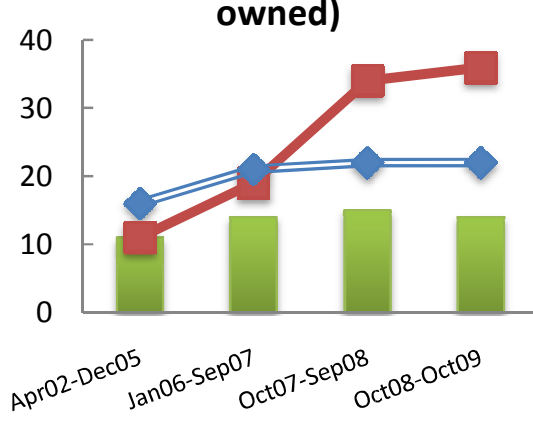

Firm E

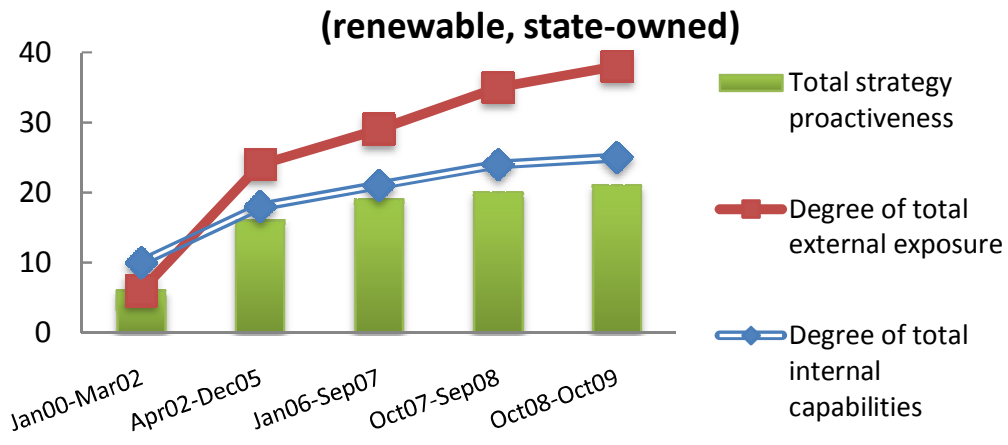


Figure 7-4 indicates that for the period April 2002 - September 2008 the strategy proactiveness of all generators increased. However, strategy proactiveness of Firms A, C and D decreased from October 2008 to October 2009, despite overall increases in both the external volatility and pressure exposure and degree of internal capabilities. This can be explained by the presence of the external regulatory uncertainty and less positive environment for renewable investment associated with the ETS delay and review (7.3.1). In contrast, during this period Firm E decided to pursue a global geothermal strategy, drawing upon its prior experience to tap into favourable international regulatory environments (6.6.5). Therefore its level of proactiveness increased. Further, due to its substantial potential ETS liabilities, from October 2008, Firm B decided to switch to renewable-only generation investments to radically reduce its emissions (6.3.5), thereby increasing its level of proactiveness.

Further, Figure 7-4 indicates that in the earlier periods (Apr2002-Sep2007), a rise in strategy proactiveness was broadly consistent with increasing internal capabilities in all the generators. In the later periods (Oct2007-Oct2009), the association between internal capabilities and strategy proactiveness was only evident within Firms B and E where more proactive environmental strategies were accompanied by a higher degree of internal capabilities. In contrast, while strategy proactiveness decreased in Firms A, C and $\mathrm{D}$, the degree of their internal capabilities stabilised. This is because despite having a high level of internal capabilities, these generators chose to reduce their strategy proactiveness to respond to regulatory uncertainty.

Additionally, qualitative analysis of interview data supports a two-way relationship between strategy proactiveness and internal capabilities. In some cases, generators possessed existing internal capabilities (e.g. top management support of renewables) which enabled the pursuit of proactive environmental strategies (e.g. renewable-only generation strategy). In turn, the choice of proactive environmental strategies facilitated the development of further internal capabilities, i.e. renewable expertise built through the implementation of large-scale renewable investment programmes. This result is consistent with the graphs in Figure 7-4 and explains why most generators experienced an increase in the level of total capabilities, when they adopted more proactive GCCPrelated environmental strategies.

Therefore, in support of P3, the generators increased the level of environmental strategy proactiveness to respond to an increase in external volatility and pressure exposure associated with the GCCPs. Additionally, the results suggest that a high level of strategy proactiveness appears to both enable and be enabled by a higher level of total internal 
capabilities (a RBT view) and that the impact of external exposure and internal capabilities on strategy proactiveness varied across generators and across different time periods. These insights are not captured in the Research Framework or P3. Figure 7-5 provides more detail on the variations in strategy proactiveness between the five generators over the five time periods from 2000 to 2009 and supports the analysis presented above.

Figure 7-5: Change in strategy proactiveness (2000-2009)

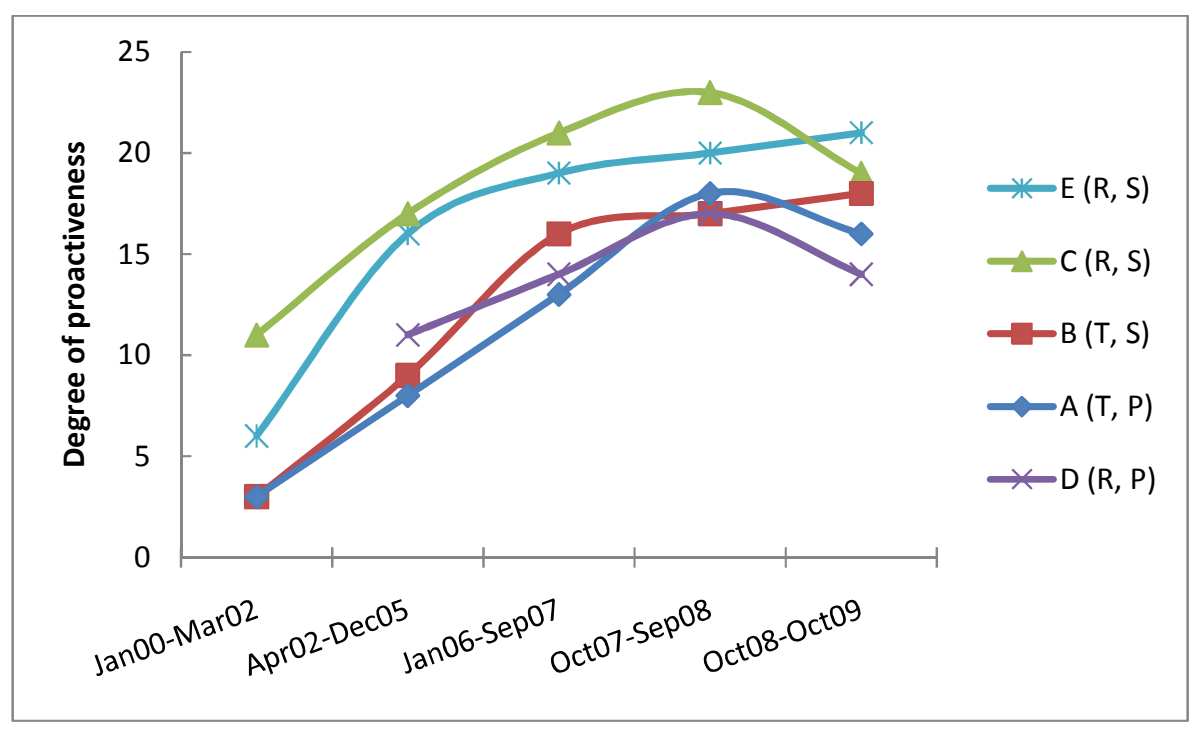

Additionally, Figure 7-5 suggests key differences exist between the generators in the degree of strategy proactiveness pursued within each period. From January 2000 to September 2008, there were two separate clusters in terms of strategy proactiveness, one comprising Firms $\mathrm{C}$ and $\mathrm{E}$ and the other including Firms A, B and D. Strategy proactiveness levels within Firms $\mathrm{C}$ and $\mathrm{E}$ were higher than within the other three generators because both perceived a high degree of GCCP-related external exposure and possessed strong internal capabilities in these earlier periods. From October 2008 to October 2009, Figure 7-5 highlights that there were major changes in strategy proactiveness within all five generators (Firms B and E increasing their proactiveness while the other three generators adopting more reactive strategies). As mentioned, this change coincides with the associated regulatory uncertainty surrounding the ETS.

Overall, all five generators perceived they were increasingly exposed to GCCP-related volatilities and pressures from 2000 to 2008. Accordingly, in response, they adopted more proactive strategies that were supported by the generators' internal capabilities. However, during the 2008-2009 period, as regulatory uncertainty dominated, the generators with significant renewable investments (A, C, D) undertook more reactive strategies. These results support P3 in that the impacts of GCCP-related external drivers, 
as reflected in the degree of external volatility and pressure exposure, lead to a change in the level of strategy proactiveness. Proposition 3 is further supported because the results suggest that inter-generator differences in strategy proactiveness are related to variations in internal capabilities, i.e. stronger capabilities enabled a generator to implement more proactive environmental strategies. This is because internal capabilities enhance generators' competitiveness, legitimacy and cost control under the GCCPs (7.4.3). However, as mentioned, P3 is not supported in that the internal characteristics appear to have no impact on strategy proactiveness. However, internal characteristics seem to affect strategic emphasis, as discussed next.

\subsubsection{Strategic emphasis}

Tables 7-3 and 7-4 highlight that the five generators adopted a range of different environmental strategies to respond to the GCCP-related volatilities and pressures. However, based on the qualitative analysis of NVivo-coded data (including evidence as presented in Chapter 6), this study found that not all these environmental strategies received equal strategic attention (or emphasis) at top management levels across different the time periods and different generators. Table 7-7 illustrates that the strategic emphasis within the five generators and how it changed from January 2000 to October 2009. Specifically, for each period, the major environmental strategies that received strategic emphasis within each generator are listed. Since the previous section (7.5.1) has highlighted and explained inter-generator differences in strategy proactiveness levels, the discussion of Table 7-7 focuses only on the key changes in strategic emphasis across the five generators over time. 
Table 7-7: Change in strategic emphasis of five generators (2000-2009) (P3)

\begin{tabular}{|c|c|c|c|c|c|}
\hline & $\begin{array}{l}\text { Jan00 - } \\
\text { Mar02 }\end{array}$ & Apr02 - Dec05 & Jan06 - Sep07 & Oct07 - Sep08 & Oct08 - Oct09 \\
\hline $\begin{array}{l}\text { Key } \\
\text { GCCP }\end{array}$ & $\begin{array}{l}\text { RMA but } \\
\text { no GCCP }\end{array}$ & $\begin{array}{l}\text { Carbon tax } \\
\text { proposal, PRE } \\
\text { scheme }\end{array}$ & Draft NZES & $\begin{array}{l}\text { Original ETS proposal } \\
\text { and legislation }\end{array}$ & $\begin{array}{l}\text { ETS delayed and } \\
\text { modified }\end{array}$ \\
\hline Firm A & $\begin{array}{l}\text { Operational } \\
\text { emphasis }\end{array}$ & $\begin{array}{l}\text { Political } \\
\text { strategy }\end{array}$ & $\begin{array}{l}\text { Political strategy, } \\
\text { Generation strategy, } \\
\text { Emissions disclosure }\end{array}$ & $\begin{array}{l}\text { Political strategy, } \\
\text { Generation strategy, } \\
\text { Emissions disclosure }\end{array}$ & $\begin{array}{l}\text { Carbon credit strategy } \\
\text { Emissions disclosure }\end{array}$ \\
\hline Firm B & $\begin{array}{l}\text { Operational } \\
\text { emphasis }\end{array}$ & $\begin{array}{l}\text { Political } \\
\text { strategy, } \\
\text { Generation } \\
\text { strategy }\end{array}$ & $\begin{array}{c}\text { Political strategy, } \\
\text { Generation strategy, } \\
\text { Emissions } \\
\text { management, } \\
\text { Emissions disclosure }\end{array}$ & $\begin{array}{c}\text { Political strategy, } \\
\text { Generation strategy, } \\
\text { Carbon credit strategy, } \\
\text { Emissions disclosure }\end{array}$ & $\begin{array}{l}\text { Generation strategy, } \\
\text { Internal restructuring, } \\
\text { Carbon credit strategy, } \\
\text { Emissions disclosure }\end{array}$ \\
\hline Firm C & $\begin{array}{c}\text { Operational } \\
\text { emphasis }\end{array}$ & $\begin{array}{l}\text { Political } \\
\text { strategy, } \\
\text { Generation } \\
\text { strategy }\end{array}$ & $\begin{array}{c}\text { Political strategy, } \\
\text { Generation strategy, } \\
\text { Retail strategy, } \\
\text { Carbon neutrality } \\
\text { strategy, } \\
\text { Carbon credit strategy } \\
\text { Emissions disclosure }\end{array}$ & $\begin{array}{c}\text { Political strategy, } \\
\text { Generation strategy, } \\
\text { Retail strategy, } \\
\text { Carbon neutrality } \\
\text { strategy, } \\
\text { Carbon credit strategy } \\
\text { Emissions disclosure } \\
\end{array}$ & $\begin{array}{l}\text { Internal restructuring, } \\
\text { Retail strategy, } \\
\text { Emissions disclosure }\end{array}$ \\
\hline Firm D & & $\begin{array}{l}\text { Political } \\
\text { strategy, } \\
\text { Generation } \\
\text { strategy }\end{array}$ & $\begin{array}{l}\text { Political strategy, } \\
\text { Generation strategy }\end{array}$ & $\begin{array}{l}\text { Political strategy, } \\
\text { Generation strategy, } \\
\text { Carbon neutrality } \\
\text { strategy } \\
\text { Retail strategy }\end{array}$ & $\begin{array}{c}\text { Retail strategy, } \\
\text { Carbon credit strategy }\end{array}$ \\
\hline Firm E & $\begin{array}{c}\text { Operational } \\
\text { emphasis }\end{array}$ & $\begin{array}{l}\text { Political } \\
\text { strategy, } \\
\text { Generation } \\
\text { strategy, } \\
\text { Customer } \\
\text { energy } \\
\text { efficiency }\end{array}$ & $\begin{array}{l}\text { Political strategy, } \\
\text { Generation strategy, } \\
\text { Customer energy } \\
\text { efficiency, } \\
\text { Internal energy } \\
\text { efficiency, } \\
\text { Retail strategy, } \\
\text { Emissions disclosure }\end{array}$ & $\begin{array}{c}\text { Political strategy, } \\
\text { Generation strategy, } \\
\text { Customer energy } \\
\text { efficiency, } \\
\text { Internal energy } \\
\text { efficiency, } \\
\text { Retail strategy, } \\
\text { Emissions disclosure }\end{array}$ & $\begin{array}{c}\text { Generation strategy, } \\
\text { Internal restructuring, } \\
\text { Carbon credit strategy } \\
\text { Retail strategy, } \\
\text { Customer energy } \\
\text { efficiency, } \\
\text { Emissions disclosure }\end{array}$ \\
\hline
\end{tabular}

It is apparent from Table 7-7 that there was an increase in the number of environmental strategies receiving management attention from January 2000 to 2008. However, from 2008 to 2009, the number of environmental strategies emphasised reduced in Firms A, C, D, and E, but not in Firm B.

From January 2000 to March 2002, since the key external pressures came from RMA resource consent requirements, all the generators focused upon complying with these requirements to manage environmental compliance costs. However, the focus was operational and no attention was required at the top management level.

From April 2002 to September 2008, through different phases of GCCP changes (from the carbon tax to the Original ETS), all generators took part in the lobbying process (political strategy) to seek to protect or enhance their economic interests. Lobbying was particularly strong from October 2007 to September 2008 when the Original ETS was proposed and legislated, as this legislation had significant implications for fuel economics and thus generators' competitiveness and production costs. Further, most generators (except Firm A) also participated in the PRE scheme to gain the carbon 
credits to assist their renewable investments (generation strategy) during the 2002-2005 period. Generation strategy continued to be the strategic focus in all five generators from 2006 to 2008. In response to such drivers as changing fuel economics, governmental and societal pressures associated with the ETS and organisational generation emission profiles, all generators had plans to pursue larger scale renewable investments (Tables 7-3 to 7-5). These investments were also enabled by multiple internal capabilities (Table 7-6).

Among the two thermal generators, Firms A had lower emissions and carbon costs and thus, decided to reduce its generation emissions by increasing its investment in renewable generation. In contrast, due to its high emissions profile and potential high carbon costs, Firm B's strategic focus was on emissions management within existing thermal plants in 2006/2007 and then it changed to buying carbon credits to prepare for future ETS compliance obligations in 2007/2008. Furthermore, to manage their social legitimacy in the face of high societal pressures associated with their generation emissions from 2006 to 2008, both generators placed strategic importance on appropriate emissions-related disclosure to manage their reputation.

In contrast, the three renewable generators emphasised different environmental strategies. From 2006, Firm C launched a green branding retail campaign and pursued CarboNZero certification to take advantage of its renewable-only assets and hence, enhancing its competitiveness. It also began trading its carbon credits to develop carbon management expertise. From 2007, Firm D followed Firm C and undertook CarboNZero certification and thus integrated carbon neutrality into its green-focused retail strategy. Firm E's management paid strategic attention to customer energy efficiency very early on (2002), developing this area with a vision to make it one of the generator's key competitive advantages. From 2006, based on its prior experience in customer energy efficiency, Firm E improved its internal energy efficiency performance to the highest industry standard and marketed its environmental responsibility. Firms $\mathrm{C}$ and $\mathrm{E}$ also employed extensive emissions-related disclosure, considering it critical to the legitimacy of their green brand. In contrast, due to its smaller size when compared to other renewable generators and little exposure to societal pressures, Firm D did not perceive emissions-related disclosure to be strategically important.

From October 2008 to October 2009, having anticipated the potential ETS changes, most generators reduced political activity and Firm D ceased to participate in the policy processes totally (6.5.4). Due to the less positive incentives proposed in the Moderated ETS and thus a reduced potential to enhance their competitiveness, three generators (A, 
C, D) progressed their renewable investments with caution. In contrast, Firms B and E underwent significant changes in their generation strategy in this period. As mentioned previously, to manage its carbon costs, Firm B shifted towards a renewable preference in its generation strategy and wrote off the value of its thermal assets (6.4.5). Firm E decided to pursue a global geothermal strategy to take advantage of its geothermal expertise and favourable international regulatory environments (6.6.5). Simultaneously, in response to the National Government's financial performance pressures, state-owned generators $(\mathrm{B}, \mathrm{C}$ and $\mathrm{E})$ undertook internal restructuring to improve operational efficiency. Additionally, the generators with ETS obligations (A, B, E) started to pay more attention to building carbon trading expertise to manage future compliance cost (carbon credit strategy). Despite the proposed ETS changes, the renewable generators $(\mathrm{C}, \mathrm{D}, \mathrm{E})$ still placed emphasis on a green-focused retail strategy in order to take advantage of their renewable-focused capabilities. Firm D differentiated itself by selling carbon credits to customers and thus enhancing its competitiveness. Additionally, Firm E continued to promote its customer energy efficiency products and services as part of its retail strategy. Similar to the previous period, emissions-related disclosure was considered important in most generators (except for Firm D), as they needed to respond to the increasing societal pressures so as to maintain their organisational legitimacy.

In summary, strategic emphasis within all the five generators changed over time. This is to respond to the varying impacts of external volatilities and pressures emerging from GCCP changes and the generators' internal characteristics and capabilities on organisational costs, competitiveness and legitimacy within each time period. This provides further support for P3 in that the impacts of GCCP-related external and internal drivers lead to changes in generators' environmental strategies. Additionally, such change in environmental strategies is motivated by a number of objectives, as discussed in the next section.

\subsection{Objectives of change in environmental strategies}

The results of analysing interview and documentary data using matrix data display indicate that there were multiple objectives underlying the change in the eleven environmental strategies found within the five generators. These objectives can be classified into three groups: competitiveness, cost control, and legitimacy. This analysis further suggests that among the eleven environmental strategies identified in Table 7-2, some were driven primarily by competitiveness, while others were driven by legitimacy or cost control objectives. Table 7-8 lists these eleven different environmental 
strategies, categorising them by the primary objective that underlie the changes in them. For each strategy, the external volatilities and pressures (Table 7-3), internal characteristics (Table 7-5), and internal capabilities (Table 7-6) found to drive it are also listed. This categorisation of environmental strategies and the inclusion of the external and internal drivers indicate why some strategies were revised to achieve particular objectives when the others were not. It is also noted that changes to generation investment and carbon credit strategies are aimed at two objectives simultaneously: cost control and competitiveness.

The analysis of strategy objectives also helps understand the relationships between the external and internal drivers and change in environmental strategies through the theoretical perspectives of TCE, IT and RBT as suggested in Chapter 4. Additionally, an understanding of these objectives explain why external and internal drivers impacted on the generators' competitiveness, costs, and legitimacy (P1, P2) and thus why changes to environmental strategies were made in response (P3). 
Table 7-8: Strategy objectives and strategy's external and internal drivers

\begin{tabular}{|c|c|c|c|}
\hline & $\begin{array}{l}\text { External volatilities and pressures } \\
\text { (institutional ones are in italics) }\end{array}$ & Internal characteristic & Internal capability \\
\hline \multicolumn{4}{|c|}{ Strategic change driven by competitiveness } \\
\hline $\begin{array}{l}\text { Generation } \\
\text { strategy }\end{array}$ & $\begin{array}{l}\text { Regulatory uncertainty and political instability } \\
\text { ETS delay } \\
\text { Cap on carbon price } \\
\text { International regulatory environment } \\
\text { Renewable investment encouragement } \\
\text { Increase in wholesale prices due to the ETS } \\
\text { Government preference for renewable investment } \\
\text { Societal pressures associated with generation } \\
\text { emissions } \\
\text { Shareholder pressures }\end{array}$ & & $\begin{array}{l}\text { Net generation volume } \\
\text { Access to renewable resource sites } \\
\text { Top management support of renewables } \\
\text { Board involvement } \\
\text { Managerial recognition of external } \\
\text { volatilities and pressures } \\
\text { Renewable expertise } \\
\text { Environmental/climate change awareness }\end{array}$ \\
\hline $\begin{array}{l}\text { Political } \\
\text { strategy }\end{array}$ & $\begin{array}{l}\text { Regulatory uncertainty and political instability } \\
\text { ETS delay }\end{array}$ & Generation emissions & $\begin{array}{l}\text { Inheritance assets } \\
\text { Renewable expertise }\end{array}$ \\
\hline Retail strategy & $\begin{array}{l}\text { Development of carbon trading expertise based } \\
\text { on PRE carbon credits } \\
\text { Changing retail competition } \\
\text { Carbon trading competition from thermal } \\
\text { generators } \\
\text { Energy efficiency market opportunities } \\
\text { Pressures from other generators } \\
\text { Greening consumer expectations } \\
\end{array}$ & & $\begin{array}{l}\text { Net generation volume } \\
\text { Inheritance assets } \\
\text { Management recognition of external } \\
\text { volatilities and pressures } \\
\text { Experience in customer energy efficiency }\end{array}$ \\
\hline $\begin{array}{l}\text { Carbon credit } \\
\text { strategy }\end{array}$ & $\begin{array}{l}\text { Renewable investment encouragement (PRE) } \\
\text { Carbon price fluctuations } \\
\text { Development of carbon trading expertise based } \\
\text { on PRE carbon credits }\end{array}$ & Financial constraints & Possession of carbon credits \\
\hline \multicolumn{4}{|c|}{ Key theoretical insight: $R B T \Leftrightarrow I T:$ competitiveness within institutional norms and resource availability } \\
\hline \multicolumn{4}{|c|}{ Strategic change driven by legitimacy } \\
\hline $\begin{array}{l}\text { Customer } \\
\text { energy } \\
\text { efficiency }\end{array}$ & $\begin{array}{l}\text { Energy efficiency market opportunities } \\
\text { RMA/ Government pressures for energy } \\
\text { efficiency and emissions reductions }\end{array}$ & & $\begin{array}{l}\text { Prior experience in customer energy } \\
\text { efficiency }\end{array}$ \\
\hline $\begin{array}{l}\text { Environmental } \\
\text { policy }\end{array}$ & $\begin{array}{l}\text { RMA/ Government pressures for energy } \\
\text { efficiency and emissions reductions } \\
\text { Greening consumer expectation } \\
\text { Sustainability industry groups }\end{array}$ & & $\begin{array}{l}\text { Top management commitment to } \\
\text { sustainability } \\
\text { Organisational culture }\end{array}$ \\
\hline $\begin{array}{l}\text { Carbon } \\
\text { neutrality } \\
\text { strategy }\end{array}$ & $\begin{array}{l}\text { Changing retail competition } \\
\text { Societal pressures associated with non- } \\
\text { generation emissions) } \\
\text { Greening consumer expectations } \\
\text { Employee pressures } \\
\text { Sustainability industry groups } \\
\text { External consultants }\end{array}$ & $\begin{array}{l}\text { Non-generation emissions } \\
\text { Organisational growth } \\
\text { Carbon-related cross functional } \\
\text { coordination }\end{array}$ & \\
\hline $\begin{array}{l}\text { Emissions- } \\
\text { related } \\
\text { disclosure }\end{array}$ & $\begin{array}{l}\text { RMA/ Government pressures for energy } \\
\text { efficiency and emissions reductions } \\
\text { Societal pressures associated with generation } \\
\text { and non generation emissions } \\
\text { Negative perception of windfall gains (ETS) } \\
\text { External consultants }\end{array}$ & $\begin{array}{l}\text { Generation emissions } \\
\text { Non-generation emissions }\end{array}$ & $\begin{array}{l}\text { Management recognition of external } \\
\text { volatilities and pressures }\end{array}$ \\
\hline \multicolumn{4}{|c|}{ Key theoretical insight: IT $\Leftrightarrow R B T$ : legitimacy within resource availability } \\
\hline \multicolumn{4}{|c|}{ Strategic change driven by cost control } \\
\hline $\begin{array}{l}\text { Emissions } \\
\text { management } \\
\text { strategy }\end{array}$ & $\begin{array}{l}\text { RMA/ Government pressures for energy } \\
\text { efficiency and emissions reductions } \\
\text { Government preference for renewable investment } \\
\text { External consultants }\end{array}$ & $\begin{array}{l}\text { Generation emissions } \\
\text { Generation emission } \\
\text { measurement and monitoring } \\
\text { Carbon-related cross functional } \\
\text { coordination } \\
\text { Technological difficulty in } \\
\text { reducing generation emissions }\end{array}$ & Experience in emissions reduction \\
\hline $\begin{array}{l}\text { Internal energy } \\
\text { efficiency }\end{array}$ & $\begin{array}{l}\text { Societal pressures associated with non- } \\
\text { generation emissions } \\
\text { Employee pressures } \\
\text { External consultants }\end{array}$ & $\begin{array}{l}\text { Non-generation emissions } \\
\text { Non-generation emission } \\
\text { measurement and monitoring } \\
\text { Organisational size } \\
\text { Organisational growth } \\
\text { Financial constraints } \\
\end{array}$ & $\begin{array}{l}\text { Organisational culture } \\
\text { Top management commitment to } \\
\text { sustainability }\end{array}$ \\
\hline $\begin{array}{l}\text { Internal } \\
\text { restructuring }\end{array}$ & Shareholder pressures & & \\
\hline $\begin{array}{l}\text { Generation } \\
\text { strategy }\end{array}$ & $\begin{array}{l}\text { Increase in wholesale prices due to the ETS } \\
\text { External technological development }\end{array}$ & $\begin{array}{l}\text { Generation emissions } \\
\text { Technological difficulty in } \\
\text { reducing generation emissions }\end{array}$ & $\begin{array}{l}\text { Risk appetite and cost consciousness } \\
\text { Relationship with majority shareholder } \\
\text { Availability of fuel supply } \\
\text { Economies of scale in renewable } \\
\text { development }\end{array}$ \\
\hline \multirow[t]{2}{*}{$\begin{array}{l}\text { Carbon credit } \\
\text { strategy }\end{array}$} & $\begin{array}{l}\text { Carbon price fluctuations } \\
\text { Development of carbon trading expertise based } \\
\text { on PRE carbon credits }\end{array}$ & $\begin{array}{l}\text { Carbon-related cross functional } \\
\text { coordination }\end{array}$ & $\begin{array}{l}\text { Possession of carbon credits } \\
\text { Relationship with majority shareholder }\end{array}$ \\
\hline & $\begin{array}{r}\text { Key theoretical insight: TCE } \Leftrightarrow I T \& \\
T C E: \text { cost } c\end{array}$ & $\begin{array}{l}\text { cost control within resource a } \\
\text { ol driven by external economic }\end{array}$ & $\begin{array}{l}\text { ability and/or institutional norms } \\
\text { tilities }\end{array}$ \\
\hline
\end{tabular}




\subsubsection{Strategic changes driven by competitiveness}

Changes in generation strategy, political strategy, retail strategy and carbon credit strategy are designed to enhance market advantages emerging from the GCCPs or to protect the generators' existing economic interests from these policies' negative impact. The data indicate that the focus of the generation, retail and political strategies were either on electricity products and services or generation investments that form the core components of their revenue and profits. The trading of carbon credits received for renewable generation could also create significant revenues (carbon credit strategy).

As evident from Table 7-8, the majority of the external drivers of changes in these strategies were economic volatilities, which, as discussed in Section 7.3.1, primarily had strategic implications for generators' competitiveness (in addition to costs). Therefore, it is unsurprising that these four strategies had the objective of enhancing or protecting generators' existing competitive advantages under the GCCP-focused environments. Furthermore, as discussed in Section 7.4.3, these strategies were enabled by many different internal capabilities, while being affected by only few internal characteristics. This partially supports a RBT view that the choice and implementation of competitiveness-driven environmental strategies are supported primarily by the internal capabilities because the deployment of such capabilities can result in a market advantage for the generators.

However, as shown in Table 7-8, these strategic changes were simultaneously influenced by a number of institutional pressures, especially in relation to generation strategies. This indicates that while pursuing strategies for competitiveness, generators also took into consideration the impacts exerted by institutional environment, i.e. the norms and expectations by organisational stakeholders such as the Government, the society and employees. Accordingly, generators were aware that their competitiveness needs to be generated and maintained within the boundaries as defined acceptable by the environmentally-concerned institutional environment. This result supports a combined RBT and IT view suggested in Section 4.3.4.2 that generators undertook differentiation strategies within a broader scope of social legitimacy to achieve and protect their competitiveness.

\subsubsection{Strategic changes driven by legitimacy}

Changes in customer energy efficiency, environmental/sustainability policy, carbon neutrality and emissions-related disclosure strategies were primarily motivated by legitimacy needs. As evident from Table 7-8, the majority of the external volatilities and 
pressures that drove these strategies were institutional. They include pressures from the Government, the society, employees, sustainability industry groups, and external consultants. In order to remain legitimate, the generators needed to satisfy and conform to the expectations and pressures of these stakeholder groups.

Therefore, the different environmental strategies were revised and changed by generators with the primary purpose to legitimise themselves to these stakeholder groups. Customer energy efficiency initiatives did not help the generators reduce costs or increase their revenue in the short term, though there were suggestions that they may enhance long-term organisational reputation and customer loyalty. Therefore, these customer energy efficiency initiatives were probably undertaken to maintain the generators' social legitimacy. Similarly, the environmental/ sustainability policy was formulated to conform to RMA compliance requirements and the pressures from the Government and sustainability industry groups, and hence protect the generators' legitimacy. Adopting a carbon neutrality strategy represents efforts to respond to climate change concerns of internal and external stakeholders (e.g. employees and consumers) and this strategy is strongly influenced by external consultants' advice and expertise. Conversely, the adoption of emissions-related disclosure was largely to demonstrate the generators' environmental responsibility in the face of increasing societal pressures associated with emissions reduction. Consequently, although having two external economic drivers, these four strategies were primarily driven by legitimacy objectives, thus providing support for an IT-based explanation (4.3.2).

These legitimacy-driven strategic changes were also associated with a small number of internal characteristics and capabilities. Carbon neutrality and emissions-related disclosure strategies were predominantly affected by internal characteristics, including generation and non-generation emissions, organisational growth, and carbon-related cross functional coordination. These internal characteristics generally led to an increase in organisational exposure to emissions-focused societal pressures, which in turn motivated generators to undertake broader-scoped environmental strategies in order to maintain legitimacy. In contrast, customer energy efficiency and environmental policy strategies were influenced primarily by internal capabilities including prior experience, top management commitment and awareness, and organisational culture. Such capabilities facilitated the implementation of these strategies and enabled the generators to maintain or enhance their legitimacy more effectively. Consequently, while the impacts of internal characteristics on legitimacy-focused environmental strategies can 
be explained from an IT-based view, the influence of internal capabilities on these strategies fits a RBT explanation. This supports a combined IT and RBT view that generators adopt some environmental strategies to preserve or further their legitimacy and their ability to do so depends on internal resource availability (4.3.4.2).

\subsubsection{Strategic changes diriven by cost control}

Changes in emissions management, internal energy efficiency and internal restructuring strategies were primarily driven by cost control objectives rather than competitiveness or legitimacy. Thermal generators, driven by their generation emissions, needed to reduce their potential ETS carbon costs through an emissions management strategy. In contrast, renewable generators undertook internal energy efficiency strategies to reduce the resources and money consumed by internal non-generation activities which was especially important if the generators were pursuing carbon neutrality programmes. Internal restructuring was conducted within state-owned generators (from October 2008) to control costs and improve operational efficiency. In addition, within some generators $(\mathrm{A}, \mathrm{B}, \mathrm{E})$, generation investment and carbon credit strategies were driven by a need to control carbon costs.

Consistent with this, Table 7-8 illustrates that internal characteristics were the dominant drivers of these cost-driven strategies. High generation emissions (high asset specificity) lead to high ETS compliance carbon costs. Carbon-related cross functional coordination and technological difficulty in reducing generation emissions (representing transactional uncertainty) increases the transactional costs associated with emissions management. Similarly, non-generation emissions (asset specificity) and organisational growth (transactional uncertainty) results in high operating costs associated with resource consumption and carbon offsets purchases. Thus, these internal characteristics caused an increase in costs which need to be controlled through the adoption of costdriven strategies. Support for a TCE perspective is therefore provided (4.3.1).

Table 7-8 also indicates that these changes in emissions management and internal energy efficiency strategies and internal restructuring were influenced by institutional pressures including those from the Government, society, consumers, employees, sustainability industry groups, shareholders, and external consultants, rather than economic ones for efficiency reasons. These pressures provided an additional incentive for generators to pursue emissions management, internal energy efficiency strategies and internal restructuring because the failure to do so will result in a loss of legitimacy for the generators to its stakeholders. This suggests that while some strategies can be 
motivated by cost control objectives, they are simultaneously designed to fit the predominant norms and expectations in the institutional environments. Consequently, this result supports a combined TCE and IT view suggested in Chapter 4 (4.3.4.1) that cost-economising decisions need to be made within institutional norms and constraints.

In contrast, cost-focused generation investment and carbon credit strategies were driven by a number of external volatilities such as changing fuel economics, external technological development and carbon market fluctuations. These external economic drivers had implications for generators' production and carbon costs (7.7.1), which led the generators to revise their generation investment and carbon credit strategies to optimise production and transactional efficiency. This result supports a TCE view in that cost control strategies are driven by external economic volatilities.

Cost control for efficiency reasons was also related to internal capabilities. A number of internal capabilities consisting of prior experience, organisational culture, and top management commitment to sustainability, enabled the generators to implement emissions management and internal energy efficiency strategies. These strategies were aimed at reducing the levels of generation and non-generation emissions and controlling carbon and operating costs respectively. Additionally, generation investment and carbon credit strategies were enabled by internal capabilities, including the availability of fuel supply, economies of scale in renewable development, having a relationship with a majority shareholder, and the possession of carbon credits. These capabilities helped reduce production and carbon costs, and enabled internal efficiency optimisation to be achieved. This supports a combined TCE and RBT view that the availability of internal resources influences the effectiveness of cost control (4.3.4.3).

\subsubsection{Relationships between external and internal drivers and strategy objectives}

The analysis of strategic change objectives shows that changes in environmental strategies made by generators were motivated by three primary objectives: maintaining/enhancing competitiveness; reducing costs; and maintaining these generators' social legitimacy in a GCCP-focused context. These differing objectives were driven by the impacts of GCCP-related external volatilities and pressures, internal characteristics and capabilities on the generators' competitiveness, costs, and legitimacy. The impacts of external and internal drivers further provide an incentive for generators to change their environmental strategies so that their effects can be managed 
(enhancing competitiveness, controlling costs, or maintaining legitimacy). Thus, further support for $\mathrm{P} 1, \mathrm{P} 2$ and $\mathrm{P} 3$ is provided.

The empirical evidence for the relationships between RBT, IT and TCE are also found (Table 7-8). Results strongly suggest that changes in competitiveness-driven strategies are made to utilise internal capabilities or resource availability and match the institutional pressures (RBT and IT). Furthermore, strategic changes for cost control are strongly driven by institutional pressures (TCE and IT) and influenced to a limited extent by internal capabilities (TCE and RBT). Additionally, some evidence is provided for the argument that strategies designed to achieve legitimacy objectives were facilitated by available internal capabilities (IT and RBT). Figure 7-6 summarises the results above and highlights the relationships and interactions between TCE, IT and RBT. This framework is consistent with Figure 4-1 (Chapter 4) which proposes that relationships exist between TCE, IT and RBT and these can be used to explain environmental strategy and MCS choice. As illustrated in Figure 7-6, the key common insight that can be made is that environmental strategies are changed within the boundaries that are defined as acceptable by institutional pressures and resource availability as represented by internal capabilities. 


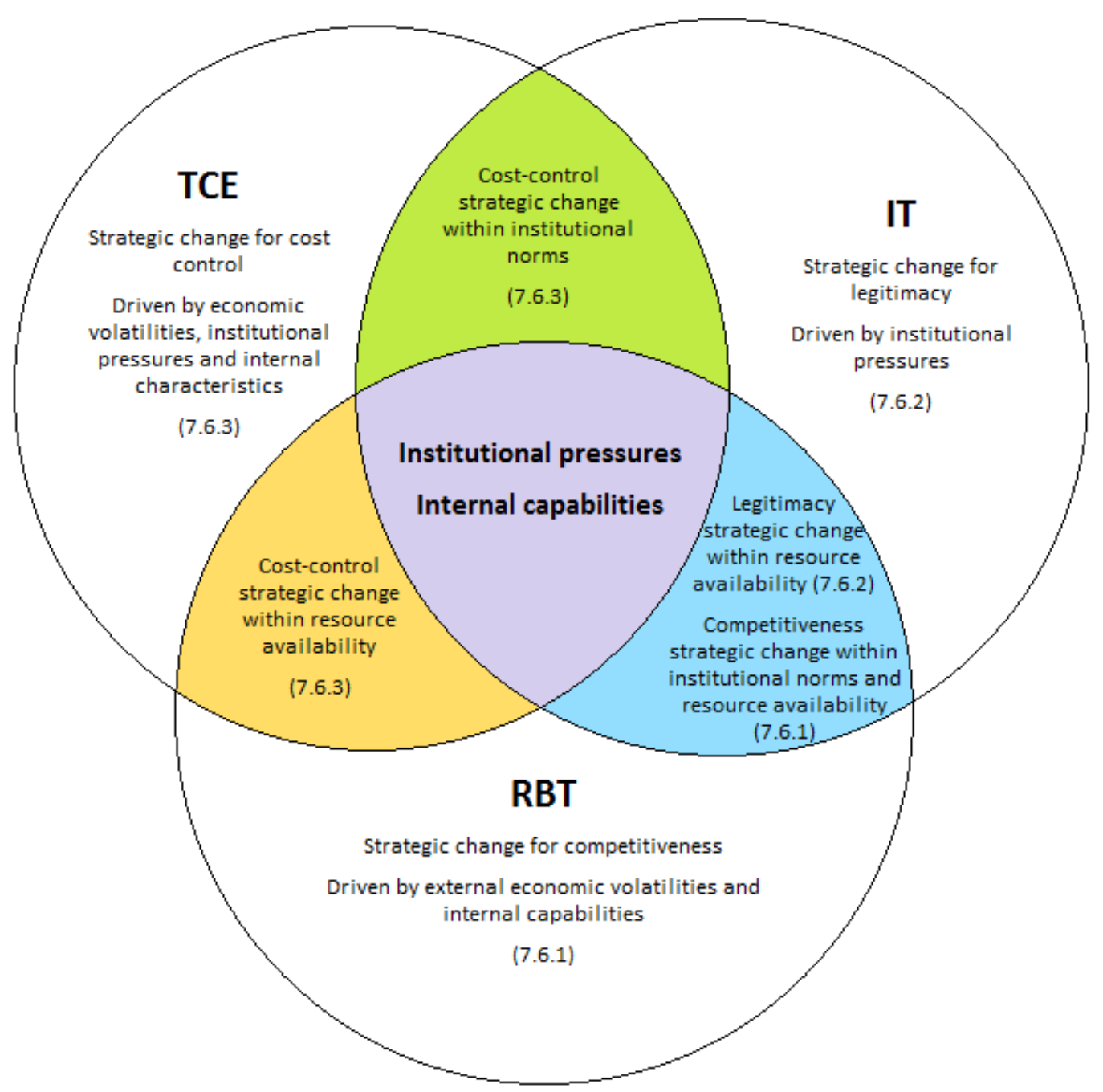

Thus, it can be argued that the generators made change to their environmental strategies for three major objectives, to: manage competitiveness; ensure cost control; and achieve social legitimacy in a GCCP-focused context. However, these objectives were not equally important across the five generators, as presented next.

\subsubsection{Variations in the importance of strategy objectives}

Analysis indicates that there were differences between the generators in the key objectives of each generator when establishing and implementing their environmental strategies, as illustrated in Table 7-9. These objectives are ranked by the frequency with which they motivated different environmental strategies within each generator. 


\begin{tabular}{|c|l|l|l|l|l|}
\hline $\begin{array}{c}\text { Ranking by } \\
\text { frequency }\end{array}$ & \multicolumn{1}{|c|}{ Firm A } & \multicolumn{1}{|c|}{ Firm B } & Firm C & Firm D & Firm E \\
\hline 1 & $\begin{array}{l}\text { Carbon cost } \\
\text { control }\end{array}$ & $\begin{array}{l}\text { Carbon cost } \\
\text { control }\end{array}$ & Competitiveness & Competitiveness & Competitiveness \\
\hline 2 & Legitimacy & Legitimacy & Legitimacy & Legitimacy & Legitimacy \\
\hline 3 & Competitiveness & Competitiveness & $\begin{array}{l}\text { Operational cost } \\
\text { control }\end{array}$ & $\begin{array}{l}\text { Transactional cost } \\
\text { control }\end{array}$ & $\begin{array}{l}\text { Carbon cost } \\
\text { control }\end{array}$ \\
\hline 4 & $\begin{array}{l}\text { Transactional } \\
\text { cost control }\end{array}$ & $\begin{array}{l}\text { Transactional } \\
\text { cost control }\end{array}$ & $\begin{array}{l}\text { Transactional cost } \\
\text { control }\end{array}$ & $\begin{array}{l}\text { Transactional } \\
\text { cost control }\end{array}$ \\
\hline
\end{tabular}

The environmental strategies adopted in thermal generators (A and B) had the similar objectives of reducing carbon costs and ensuring legitimacy. The strategic focus on carbon cost control is self-explanatory, because these two generators had the highest levels of generation emissions and thus carbon costs in the industry under the ETS. Maintaining legitimacy was also very important for them since they were exposed to negative societal perceptions associated with their generation emissions. Additionally, competitiveness had some importance as an objective for generation, retail and political strategies within both generators. For Firm A, strategic choices were made to develop and reinforce its competitive advantage in geothermal energy. In contrast, Firm B's strategies were undertaken to protect its traditional competitive advantage in thermal generation.

The predominant strategic choice in the environmental strategies adopted by renewablebased Firms C, D and E was differentiation in order to maintain or enhance market competitiveness. Having a substantial renewable asset base and expertise, they developed environmental strategies to take advantage of the potential positive opportunities associated with the GCCPs. Two renewable generators (C \& D) were exposed to societal pressures associated with non-generation emissions and hence they needed to demonstrate their climate change responsibility. Therefore, a number of strategies, e.g. customer and internal energy efficiency initiatives and emissions-related disclosure, were undertaken to ensure or enhance their social legitimacy. Firm E, although a low thermal emissions profile, was still seen by the public as having thermal generation. It responded to these societal pressures through adopting a sustainability policy and emissions-related disclosure.

Operational cost and carbon cost control were of some importance within Firms $\mathrm{C}$ and $\mathrm{E}$ respectively. Firm $\mathrm{C}$ also adopted differentiation strategies through implementing broad-scoped internal energy efficiency initiatives and a carbon neutrality programme to reduce the operating costs associated with its rapid organisational growth. Carbon cost was a concern in Firm $\mathrm{E}$ due to its low emissions profile and the sufficient carbon 
credits it granted from the PRE scheme to meet its ETS compliance obligation. Therefore, it adopted an emissions management strategy, but did not assign significant strategic attention to that strategy.

Transactional costs arising from GCCP-related external and internal drivers, e.g. carbon market fluctuations, emissions measurement and monitoring, and carbon trading transactions, presented some concern for all generators. Consequently, a number of environmental strategies (i.e. emissions management, internal energy efficiency and carbon credit strategies) were established with transactional cost control as one of the objectives. However, it is noted that transactional cost control is of secondary importance, as opposed to carbon cost and operating cost control which were the primary objectives that drove the adoption of these strategies.

\subsection{Summary}

To summarise, results presented in this chapter provide empirical support for P1, P2 and P3. External volatilities and pressures can be economic or institutional in nature, with economic volatilities affecting the generators' competitiveness and costs, and institutional pressures having implications for their legitimacy (P1). Internal characteristics had an impact on the generators' costs and legitimacy while internal capabilities enhanced generators' competitiveness and legitimacy and enabled better cost control (P2).

Additional insights are gained regarding the external and internal drivers. Results indicate that the internal drivers moderated the generators' exposure to external drivers. Internal characteristics increased the negative impacts of the external drivers on the generators, e.g. by imposing additional costs and exposing them to stronger governmental and societal pressures. In contrast, internal capabilities enhanced the GCCP-related positive impacts by providing an advantage for the generators to control cost, and enhance competitiveness or legitimacy in the face of the external drivers. New drivers are found in addition to those suggested in the Research Framework. They are: regulatory and government pressures (external drivers); changing competition (external driver); organisational growth (internal characteristic); technological and financial constraints (internal characteristics); and ownership-related capabilities (internal capabilities).

In support of P3, this study identified eleven different environmental strategies that generators changed in response to GCCP-related external and internal drivers. External 
volatilities and internal capabilities are the most predominant influence of generation, retail, political and carbon credit strategies. Institutional pressures exerted the most significant impacts on emissions-related disclosure, carbon neutrality, customer energy efficiency, environmental policy, and internal restructuring strategies while internal characteristics primarily affected emissions management and internal energy efficiency strategies (Table 7-8).

Additionally, changes in environmental strategies are reflected in the level of strategy proactiveness and changes in strategic emphasis. The level of strategy proactiveness of generators increased over time to respond to the increasing level of GCCP-related external exposure. The more proactive strategies were generally supported by a higher level of internal capabilities (P3). Further, top management assigned differing levels of emphasis to environmental strategies over time, corresponding to the changing GCCPs and associated volatilities and pressures experienced by the generators, and the impacts exerted by internal characteristics and capabilities (P3).

In addition to supporting the propositions (P1, P2, and P3), the results suggest that changes in different environmental strategies were made to achieve the objectives of maintaining competitiveness, controlling costs or ensuring legitimacy. However, each generator focused on one (or two) objective(s) in choosing and implementing their environmental strategic changes. The results also provide evidence to support the use of three theoretical perspectives (TCE, IT and RBT) in explaining the impacts of external and internal drivers on generators' environmental strategies, as well as the dynamics and variations in the use of these strategies across time and generators.

The environmental strategies undertaken in response to GCCP-related external and internal drivers in turn had significant implications for organisational MCS. The next chapter - Chapter 8 presents and discusses the results of analysis into organisational MCS design, use and objectives that were adopted by the generators to implement their GCCP-related environmental strategies. 


\section{Chapter 8: Change in organisational MCS}

\subsection{Introduction}

The results presented in Chapter 7 provide support for P1, P2, and P3 and suggest that generators modified eleven environmental strategies in response to the GCCP-related external and internal drivers. These environmental strategies were underpinned by three objectives (competitiveness, cost control, and social legitimacy) and used differently across generators and time, as reflected by the dynamics of and variations in the level of strategy proactiveness and changes in strategic emphasis. These environmental strategies and their associated aspects (i.e. proactiveness and emphasis) are likely to have different requirements and implications for organisational MCS, to ensure that these strategies are effectively planned and implemented. This chapter analyses MCS design, use and objectives in the five generators to understand how their MCS changed to support GCCP-related environmental strategies. This addresses the study's research question and assesses the validity of $\mathrm{P} 4$ : The change in environmental strategies requires corresponding modifications in organisational MCS.

The results in this chapter are based on the analysis of the evidence presented in Chapter 6 and other relevant interview and documentary data. Specifically, the classification of controls and control systems into their relevant MCS component types and groups are achieved using data mapping based on Excel-based matrix data displays, as discussed in Chapter $5(5.7 .2)^{12}$. The tables and figures (i.e. charts) presented are derived from the analysis and resulting matrix displays. Where applicable, a reference to a section in Chapter 6 will be made to highlight the source of evidence that supports a key argument or finding (e.g. 6.2.1). Relevant figures or sections in Chapter 7 that link MCS changes to the changes in environmental strategies will also be mentioned (e.g. 7.5).

As outlined in Chapter 4 (4.4.5), this study considers MCS to be the collection of controls and control systems and their use by managers to ensure the adaptation of organisations to the environments and the achievements of organisational objectives. Therefore, the term "control" and "control system" will be used interchangeably with

\footnotetext{
12 These matrix displays are available on request provided they do not breach the confidentiality agreements with the interviewees.
} 
"MCS" throughout this chapter. To further the analysis in this chapter, controls and control systems related to environmental strategies were firstly identified from interview and documentary data and classified into appropriate MCS component types and groups. For the methods used to analyse MCS design, use and objectives, see Chapter 5 (5.7.2.4).

This chapter is organised as follows. The next section examines MCS design and use as captured in different MCS component types or groups that were identified from the data, and discusses the changes and cross-firm variations in the degree of use of each MCS component type or group from 2000 to 2009. This is followed by an analysis of the objectives underlying the design and use of organisational MCS and inter-generator differences in the choice of key MCS objectives. Lastly, a summary of the chapter is presented.

\subsection{Change in MCS design and use}

The analysis of interview and documentary data suggests that one environmental strategy can be supported by multiple control and control systems. Similarly, one control or control system can support multiple environmental strategies. In this study, MCS design is reflected in the existence or establishment of particular controls and control systems, as listed in Tables 8-1 and 8-2, while MCS use refers to the employment of these controls and control systems to implement or plan environmental strategies. Additionally, this study examines the degree of use of a particular control/ control system at different levels of management. As discussed below, the degree of MCS component use depends on the level of strategy proactiveness and/or strategic emphasis placed on one or some environmental strategies in a given time period.

A diverse range of controls and control systems were found that relate to GCCP concerns and the eleven environmental strategies that were undertaken by the generators in response. Where possible, the MCS components are classified into the four main types, following Simons (1991) categories, as discussed next. However, some controls and control systems did not fit these categories, as presented in Section 8.2.2.

\subsubsection{Simons (1991) control systems}

As outlined in Chapter 3 (3.5.2), the four MCS component types suggested by Simons (1991) are: boundary controls, belief systems, diagnostic controls and control systems and interactive control systems. The controls and control systems identified from 
Chapter 6 evidence and matrix data displays are classified into their component types as shown in Table 8-1. Each type is discussed below.

Table 8-1: Simons (1991) types of controls and control systems

\begin{tabular}{|l|l|l|}
\hline Boundary controls & $\begin{array}{l}\text { Diagnostic controls and control } \\
\text { systems }\end{array}$ & Interactive control systems \\
\hline $\begin{array}{l}\text { Environmental/ sustainability policy } \\
\text { Carbon-related spending limits }\end{array}$ & Generation emissions measures & Non-generation emissions measures \\
Required rate of return & Emissions reduction targets & $\begin{array}{l}\text { ETS position information system } \\
\text { Carbon price monitoring system }\end{array}$ \\
Sustainable procurement policy & Emissions monitoring system & Investment planning systems (with \\
Energy efficiency guide & Emissions key performance indicators & integration of climate change issues) \\
Separation of responsibilities & Carbon-related budgetary controls & $\begin{array}{l}\text { Internal communication system } \\
\text { Profit planning system }\end{array}$ \\
\hline Belief systems & Link to managerial compensation & Use of accounting in political strategy \\
\hline Mission statement & Integration of emissions measures in & \\
Unwritten corporate values & balanced scorecard & Budgetary controls \\
\hline
\end{tabular}

\section{Boundary controls}

Boundary controls define the limits, practices and rules to be expected and the risks to be avoided by organisational members in conducting organisations' operations and activities (Simons, 1991) (3.5.2). An environmental policy highlights the approach a generator undertakes in relation to environmental issues and the mechanisms and practices through which they limit their operational impacts on the natural environment. A sustainability policy is wider, prescribing the processes/mechanisms by which a generator should implement its environmental strategies to ensure the achievement of economic performance in a sustainable manner within a carbon emissions-constrained context. In some generators, carbon-related spending limits are specified to ensure that adequate control is in place to avoid undertaking climate change projects (e.g. carbon credit purchases or internal energy efficiency initiatives) whose costs exceed the organisation's risk appetite (6.2.5). A required rate of return on investment is a popular boundary control for all generators, although it appears to be set higher in publiclylisted generators because of the capital market pressures (6.2.3). This control ensures that the generation projects that are pursued potentially provide a sufficient return to the generators' shareholders. Sustainable procurement policy and energy efficiency guides are mechanisms that help organisational members change their procurement practices and resource consumption behaviour to ensure consistency with the generators' internal energy efficiency strategies. Furthermore, there was a clear separation of responsibilities between the management, measurement and reporting of emissions and carbon market fluctuations and costs in some generators (6.4.4). Each of these responsibilities belonged to a separate department or team. 
Figure 8-1 illustrates the change in the total degree of use of the boundary controls of the five generators from 2000 to 2009. It is evident that the degree of boundary control use has increased over the period. However, the degree of increase varies across the generators, as explained next.

Figure 8-1: Use of boundary controls (2000-2009)

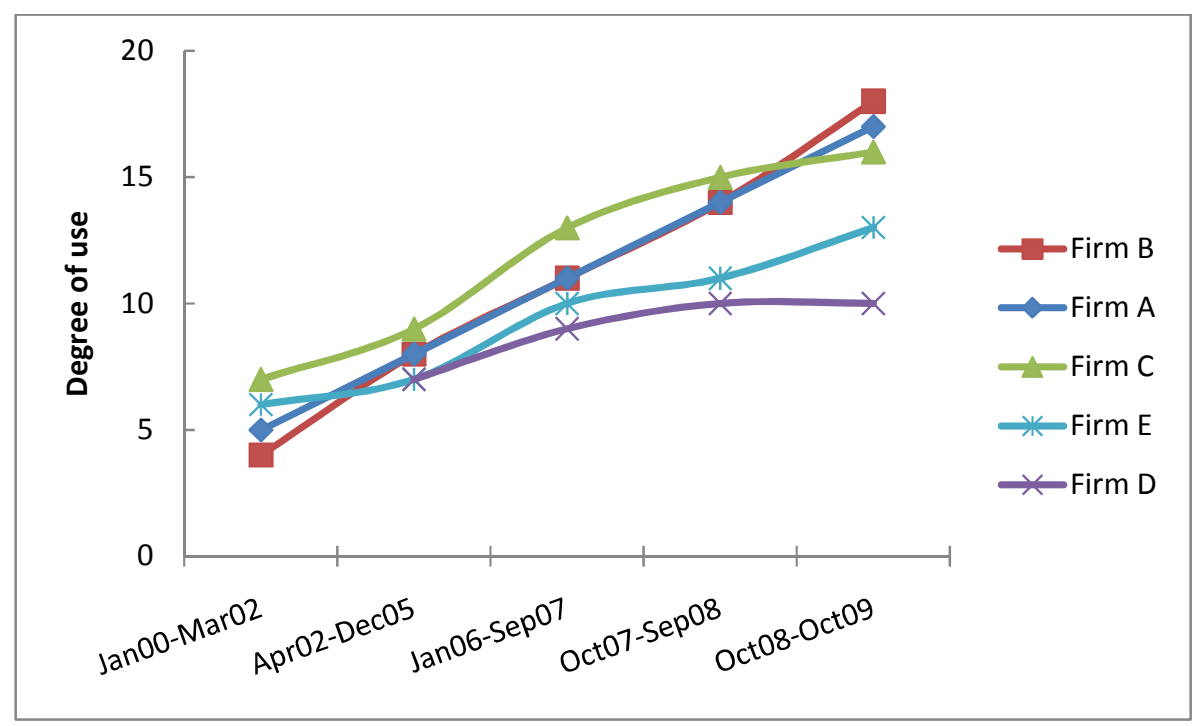

From 2000 to 2002, Firm C and Firm E had a higher use of boundary controls than the other generators, because they were the first to adopt a sustainability policy, and sustainable procurement and energy efficiency guides. Firm $\mathrm{C}$ also had the highest use of boundary controls in all periods (except from October 2008 to October 2009), as their environmental strategies were more proactive and wider in scope than other generators (Figure 7-5). This led to Firm $\mathrm{C}$ employing all the boundary controls discussed above, while other generators only used some of these controls. Interestingly, thermal-based Firms A and B, were almost identical in their use of boundary controls over the different periods, mainly because both generators had high carbon emission costs under the GCCPs. Their boundary control use grew at a consistent rate from 2000 to 2008 , reflecting their increasing emphasis on the carbon credit and emissions management strategies and thus, these control types as the generators prepared for their ETS obligations. From October 2008 to October 2009, due to an increase in strategic emphasis on renewable generation investment, Firm B integrated its environmental policy within the strategic planning process. This made emissions reduction a critical factor in its renewable investment decisions.

Since April 2002, Firms E and D have lagged behind the other generators. Firm E's carbon credit and emissions management strategies did not receive much top 
management attention as in Firms A, B, and C. Similarly, Firm D didn't place much strategic emphasis on internal energy efficiency initiatives throughout the period (Table 7-7). This led to only an operational use of boundary controls, which explains their low level of total use within Firms E and D.

\section{Belief systems}

Belief systems work as informal control systems within the generators that potentially affect the behaviour of organisational members. They can be written documents (i.e. a mission /vision statement), or unwritten values and common understanding (i.e. "unspoken" corporate culture) (3.5.2). The degree of use of belief systems in the five generators is illustrated in Figure 8-2.

Figure 8-2: Use of belief systems (2000-2009)

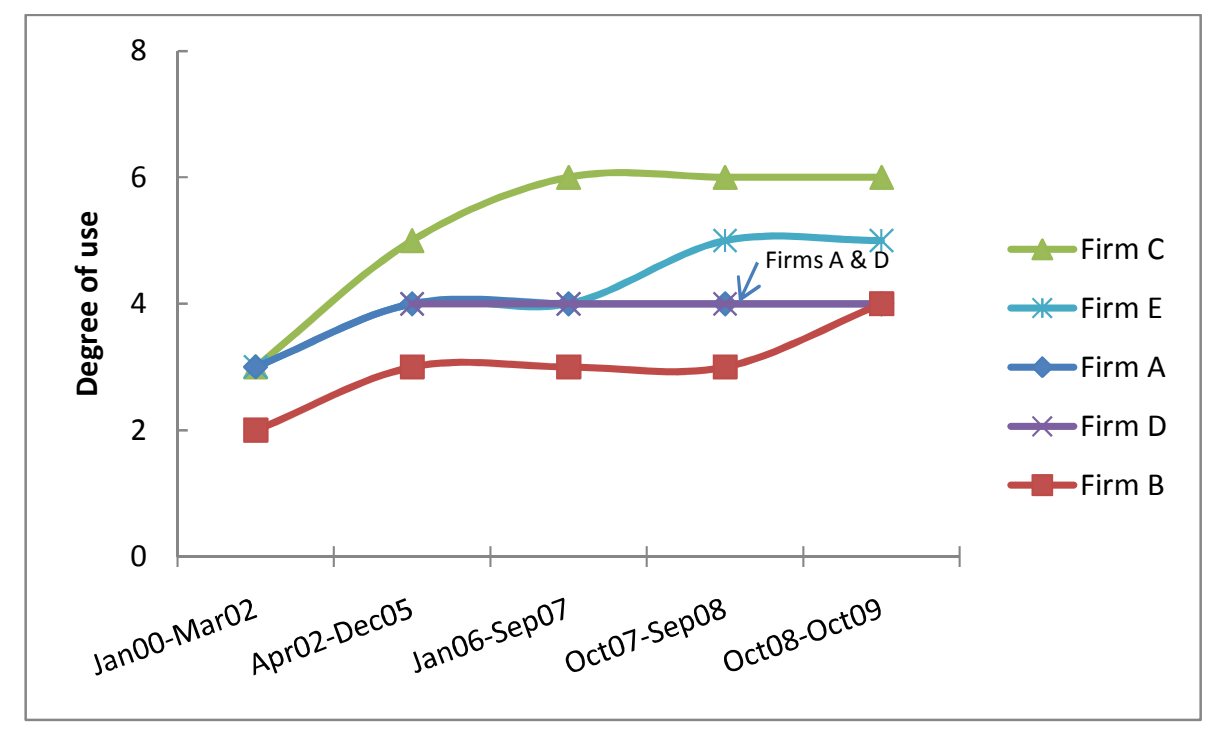

Firm $\mathrm{C}$ had a corporate culture of innovation and high environmental awareness that enabled it to undertake proactive renewable investments and internal energy efficiency strategies. Its vision was to "become a leader in climate change issues" and this vision was formalised into a mission statement that was communicated and discussed frequently at operational and strategic levels to support its renewable-only generation investment strategy (6.4.2). This explains why the degree of use of belief systems in Firm C was higher than other generators. Similar to Firm C, Firm E's corporate values were built around environmental responsibility (6.6.2). However, during the earlier periods, such values were only important at an operational level as they enabled more effective implementation of its internal energy efficiency initiatives. However, in the later periods, these values were incorporated into the strategic planning process as Firm 
E moved from a diversified generation strategy to a renewable-focused one. Thus, the degree of belief system use has increased from October 2007.

In contrast, Firms A and D, being publicly listed generators, had a similar corporate culture that focused upon financial performance and cost control (6.2.3; 6.5.2). This corporate culture strongly featured in the formulation and implementation of generation investment strategies, resulting in a similar degree of belief system use in Firms A and D (Figure 8-2). This culture also influenced the design and use of other control systems, such as the budgetary controls for carbon trading managers and the formal separation of duties in relation to environmental strategy implementation. Conversely, Firm B had the lowest level of belief system use before 2008. However, since October 2008, Firm B revised its mission statement to focus on making an adequate return for the shareholders under an ETS and used this control interactively to guide its generation investments (6.3.5). The degree of use of belief systems in Firm B has converged with that of Firms A and D, reflecting similar financially-focused belief systems.

\section{Diagnostic controls and control systems}

In order to monitor and control the implementation of emissions management and internal energy efficiency strategies, the generators designed and used an array of diagnostic controls and control systems (Table 8-1). Emissions measures were chosen for generation and non-generation activities. Firms A, B and E measured total carbon generation emissions from their thermal plants for RMA compliance purposes and as part of GCCP-related emissions management strategies. However, the scope of measurement for non-generation emissions varied across the five generators. Firm $\mathrm{C}$ measured emissions across the total electricity product lifecycle to support its proactive internal energy efficiency and carbon neutrality strategies (6.4.5). In contrast, the other generators only measured emissions for some business areas and activities (e.g. 6.2.4; 6.5.3). Additionally, all five generators adopted emissions reduction targets, though the difficulty level of these targets varied across the generators. Emissions monitoring systems were updated or replaced to ensure accurate measurement and regular internal reporting of emission levels and the performance of specific emission reduction initiatives. Emission key performance indicators were adopted to enable top managers to assess the performance of the chosen environmental strategies. Firms A, B and C planned future budgetary controls for carbon trading and the linking of emissionsrelated performance to managerial performance evaluation and compensation (carbon credit strategy) $(6.2 .5 ; 6.4 .4)$. Further, Firm C integrated emissions measures into the 
organisation-wide balanced scorecard to enable more effective reduction of nongeneration emissions (6.4.4). To improve operational efficiency and lift financial performance, state-owned generators $(\mathrm{B}, \mathrm{C}, \mathrm{E})$ also adopted tight budgetary controls as part of internal restructuring from October 2008. Figure 8-3 shows the change in the use of diagnostic controls and control systems in the five generators.

Figure 8-3: Use of diagnostic controls and control systems (2000-2009)

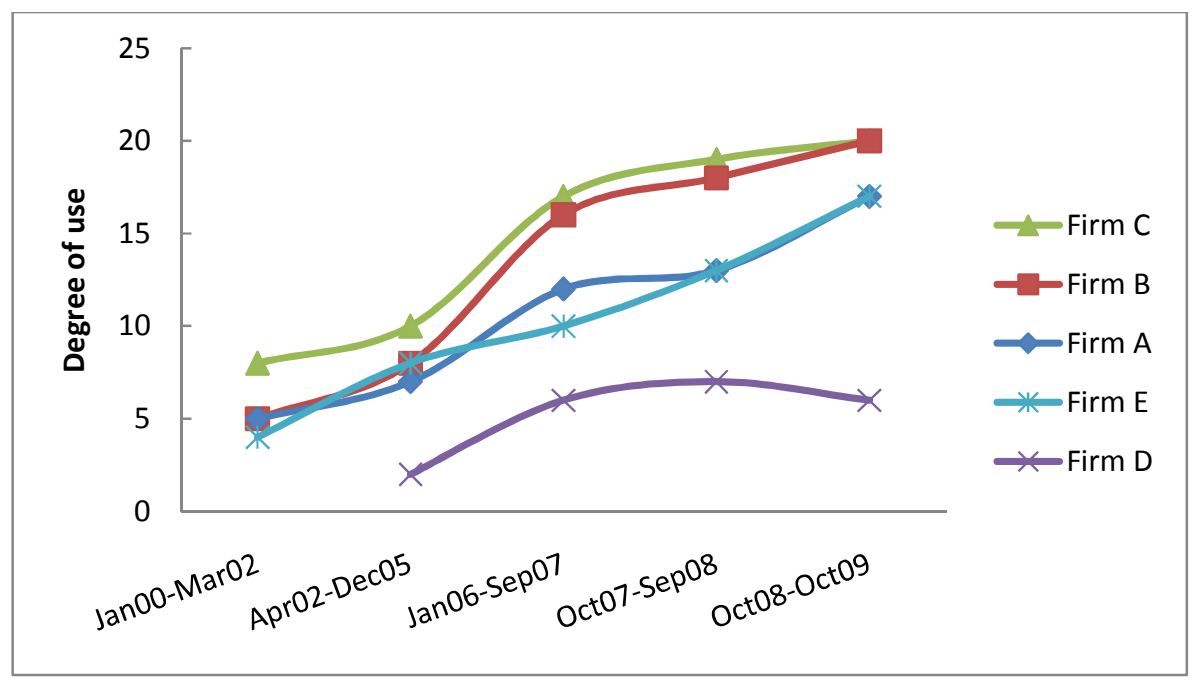

Firm $\mathrm{C}$ ranked the highest in all the periods because it established a wider range of diagnostic controls and control systems to support its proactive internal energy efficiency strategy. Furthermore, Firm C used this MCS component type more intensively over time to ensure the effective reduction of non-generation emissions. In contrast, Firm B's strategic focus is on emissions management and carbon credit strategies. Therefore, it adopted a wide range of diagnostic controls and control systems and used them intensively to reduce its generation emission levels and carbon costs, which are the highest among the five generators. From April 2002 to September 2008, Firm B experienced a higher rate of increase in the diagnostic use of MCS components than the other firms with thermal plants (A and E), which have a lower level of generation emissions. By October 2009, Firms B and C had converged in their use of diagnostic controls and control systems. This is consistent with the fact that these two generators had the highest level of generation emissions (B) and non-generation emissions (C) (Table 7-1). Additionally, both firms experienced an increase in budgetary controls due to internal restructuring (Oct2008-Oct2009).

There was also an upward trend in the number and use of diagnostic controls and control systems in Firms A and E, as these generators updated their emissions 
monitoring systems and introduced budgetary controls for carbon trading in preparation for an ETS. Additionally, Firms A and E have experienced a converging level of diagnostic control use since October 2007. However, closer examination of the data suggests that Firms A and E differed in the level of use assigned to specific diagnostic controls. Due to its higher emissions levels, Firm A placed importance on controls related to generation emissions, while Firm E considered the management of nongeneration emissions more critical. Similar to Firms B and C, from October 2008, stateowned Firm E also increased the use of budgetary controls due to internal restructuring. Thus, the difference between Firms A and E in diagnostic MCS component use is consistent with the strategic emphasis associated with each firm's emissions profile and ownership structure. In contrast and consistent with its limited-scope internal energy efficiency and carbon neutrality strategies, Firm D ranked the lowest in its use of diagnostic MCS components, although this use increased between 2002 and 2008 to reflect more managerial attention on these strategies. However, Firm D's degree of MCS diagnostic use decreased during October 2008-2009 due to the cancellation of its carbon neutrality programme.

\section{Interactive control systems}

There are a number of control systems that the top managers used interactively to engage with lower levels of management and employees to ensure internal awareness of environmental strategies and to generate innovative strategic initiatives (Simons, 1991). As explained by Simons (1991), a control system can be used diagnostically in some periods, but may become interactive controls or control systems in others. MCS is used interactively within the five generators to support primarily their generation investment, retail, political and carbon credit strategies.

In Firms $\mathrm{B}$ and $\mathrm{C}$, the emissions monitoring systems were used diagnostically prior to 2006. However, they were used interactively post-2006, at different management levels and cross-functionally, to increase staff understanding of potential GCCP impacts and to encourage behavioural change and new strategic ideas $(6.3 .3 ; 6.4 .3)$. Further, since 2007, top management in the thermal generators (A, B) have used ETS position information systems (i.e. systems that monitor a generator's ETS carbon costs) and carbon price monitoring systems interactively to assess these organisation's financial exposure to the upcoming ETS and thus revise generation investment and carbon credit strategies appropriately (6.2.4 and 6.3.4). 
Additionally, climate change issues increasingly became critical considerations in investment planning systems. To ensure the viability of their generation investments, all generators had to estimate the potential ETS carbon costs arising from proposed thermal plants or the avoided emissions and costs for renewable projects, as well as the acceptability of such plants from a societal perspective (e.g. 6.3.5; 6.6.3). Further, internal communication systems were used both formally (e.g. strategy meetings) and informally (e.g. internal newsletters and networking) in most generators to promote staff morale and encourage employees' behaviour alignment with their chosen generation and retail strategies (6.2.3). Additionally, due to the recent Governmental performance pressures, the state-owned generators $(\mathrm{B}, \mathrm{C}, \mathrm{E})$ extensively used their profit planning systems to generate creative and innovative strategy ideas to help the organisations improve their economic performance (e.g. 6.4.5). Furthermore, in their political strategy, accounting-based techniques and analyses were used interactively by some generators to advocate or argue against particular GCCPs (e.g. 6.3.4). This accounting use was primarily driven by the generators' self interests and biases, i.e. to protect the viability of their existing generation plants and development projects.

Figure 8-4 illustrates that all the generators increasingly used their MCS components interactively from 2000 to 2008 . This is consistent with the rising level of proactiveness displayed by all five generators in relation to their generation investment, retail and political strategies during this period (Figure 7-5). Proactive strategies required the active participation of multiple organisational functions to enable the generators to respond to the proposed GCCPs before they are introduced. Therefore, an increase in interactive MCS use is required to encourage input from employees and management throughout the organisation into strategy planning and implementation processes. 


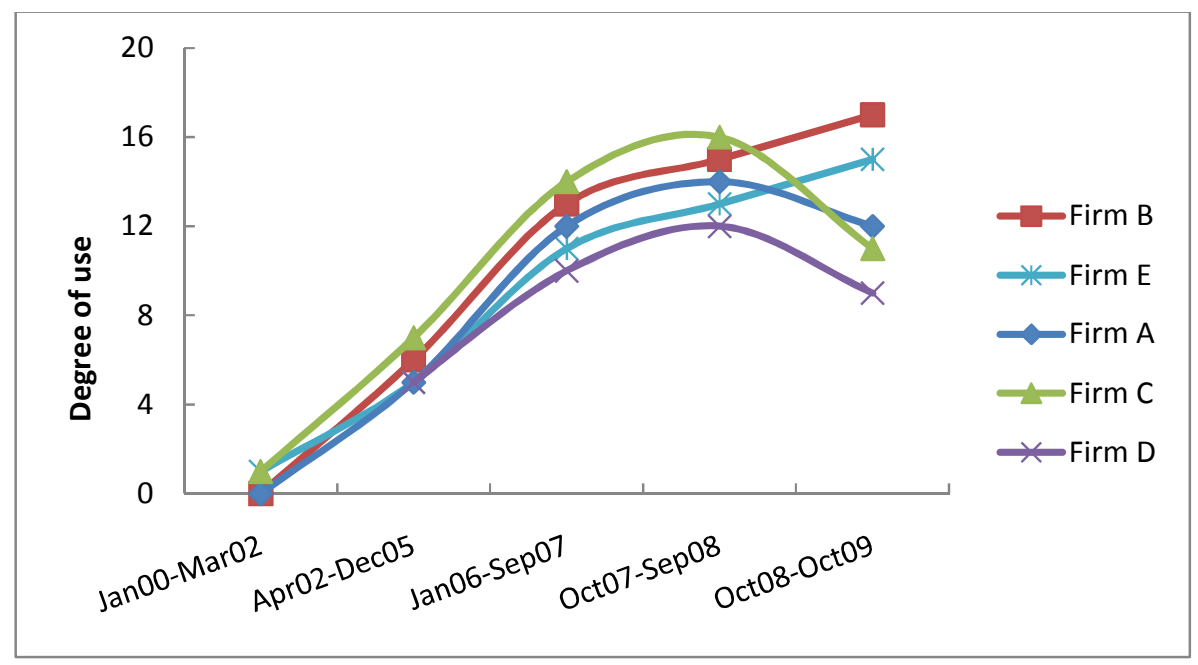

The five generators display a relatively similar degree of interactive MCS use up until December 2005 (before the carbon tax cancellation). This generally reflects little strategic attention being paid to these controls and control systems and the lack of integration of GCCP-related information into strategy planning process within all five generators ${ }^{13}$. However, the degree of interactive MCS use began to diverge from January 2006. Firm C ranked the highest in all the periods, except for October 2008October 2009. This high ranking is consistent with its early pursuit of a renewable-only generation investment strategy, a green-focused retail strategy and a carbon credit strategy to take advantage of GCCP-related positive impacts. Firm B followed Firm C closely in its interactive MCS use, reflecting its proactiveness into integrating emissions and carbon information into its strategy planning process to mitigate GCCP negative impacts on organisational performance.

From October 2008, Firms A, C and D substantially reduced the use of interactive control systems because of the decreased level of proactiveness of their generation investment and political strategies (Figure 7-5). Additionally, Firm $\mathrm{C}$ reduced the scope of its carbon trading business, thus experiencing a higher decrease in interactive MCS use than Firms A and D. In contrast, consistent with their change in generation strategy and higher level of strategy proactiveness, there was increasing interactive MCS use in Firms B and E from October 2008 (7.5.1). To facilitate this strategic change, top management in these generators used the profit planning, investment planning and internal communication systems more interactively to encourage input throughout the organisation into the strategic planning process. Consequently, by October 2009, Firms

\footnotetext{
${ }^{13}$ Evidence from Chapter 6 sections that cover the periods from Jan2000 to Dec2005, e.g. 6.2.1 and 6.2.2.
} 
$\mathrm{B}$ and $\mathrm{E}$ ranked the highest in terms of their interactive MCS use, followed by Firms A, $\mathrm{C}$, and D.

\subsubsection{Additional controls and control systems}

The analysis of documentary and interview data through matrix data display also indicates additional control systems that do not fit Simons (1991) four MCS component types. These additional control systems serve four main purposes: to coordinate different organisational functions to implement environmental strategies; to enable the development of a competitive advantage; to monitor external risks; and to legitimise to external stakeholders. Therefore, they are classified into four types labelled: crossfunctional controls and coordinating systems; enabling controls and control systems; risk monitoring systems; and legitimising controls and control systems, as given in Table 8-2. Each of these will be discussed below.

\section{Table 8-2: Additional types of controls and control systems}

\begin{tabular}{|c|c|}
\hline $\begin{array}{l}\text { Cross-functional controls and coordinating } \\
\text { systems }\end{array}$ & Enabling controls and control systems \\
\hline $\begin{array}{l}\text { Database sharing } \\
\text { Inter-system linkages } \\
\text { Internal coordination systems for energy } \\
\text { efficiency } \\
\text { Formal cross-functional reporting systems } \\
\text { Inter-firm controls and trust }\end{array}$ & $\begin{array}{l}\text { Use of HRM system to acquire expertise in carbon } \\
\text { neutrality and sustainability or carbon trading } \\
\text { Budget for customer energy efficiency } \\
\text { Use of HRM system to develop renewable expertise } \\
\text { Budget for climate change initiatives } \\
\text { Budget for carbon trading }\end{array}$ \\
\hline Risk monitoring systems & Legitimising controls and control systems \\
\hline $\begin{array}{l}\text { Climate change risk register } \\
\text { Carbon market monitoring system } \\
\text { External fuel economics monitoring system } \\
\text { Policy oversight system } \\
\text { Competitors' action monitoring system } \\
\text { External organisations' political agenda } \\
\text { monitoring system } \\
\text { Customer demand and expectation database }\end{array}$ & $\begin{array}{l}\text { External disclosure of emissions } \\
\text { Sustainability reporting } \\
\text { Customer energy efficiency information systems } \\
\text { Audit of annual reports' sustainability content } \\
\text { External audit of emissions inventory } \\
\text { External awards/accreditation for EMS } \\
\text { Carbon neutrality certification process }\end{array}$ \\
\hline
\end{tabular}

\section{Cross-functional controls and coordinating systems}

Cross-functional coordinating systems ensure coordination and cooperation between different functions to achieve the common objectives of the environmental strategies, as well as allow for between-function supervision and oversight to reduce/avoid local opportunism and manipulative behaviour. The primary environmental strategies supported by the cross-functional MCS components are internal energy efficiency, emissions management, and carbon credit strategies.

In the thermal generators (A and B), there was database sharing between different functional areas responsible for implementing emissions management and carbon credit strategies (6.2.5). Firm E maintained a separate database system within each function, 
but developed inter-system linkages to ensure that managers and staff could acquire the information they need from other functions (6.6.5). Generators also used different internal coordination systems to implement their internal energy efficiency strategies. Formally, one generator (Firm C) assigned a centralised and dedicated team with fulltime staff to collate information and coordinate initiatives between different functions (6.4.3). In other generators (Firms $\mathrm{A}$ and $\mathrm{E}$ ), such coordination was done informally through decentralised cross-functional teams who considered energy efficiency just as an add-on responsibility to their full-time functional jobs (6.6.2). In most generators (A, $\mathrm{B}, \mathrm{C}$ and $\mathrm{E})$, a strong emphasis was placed on the formal cross-functional reporting of information such as the number of carbon credits on hand, carbon credit revenue or purchases, and generation and non-generation emissions (e.g. 6.4.4). Such reporting promoted transparency and supervision among different functions as well as provided senior managers with information so they could assess the achievement of environmental strategies.

Additionally, some generators (A, B, E) used inter-firm controls because they had entered into outsourcing relationships, partnerships and joint ventures in relation to thermal investment projects or carbon credit purchases. These inter-firm controls include mechanisms such as the sharing of information systems, and inter-firm disclosure of relevant performance measures to enable mutual learning and supervision. Trust also acted as a mutual control where the generators selected the partners with whom they had previous or on-going business relationships.

Figure 8-5: Use of cross-functional controls and coordinating systems (2000-2009)

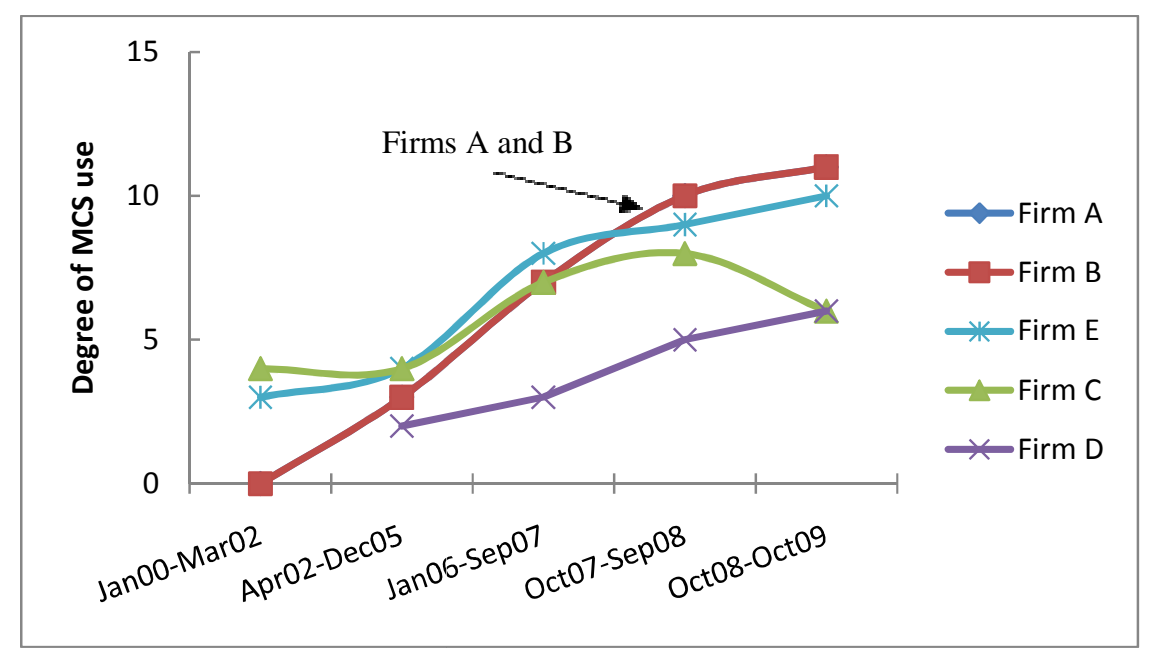

Figure 8-5 shows changes in the degree of use of cross-functional controls and control systems in the five generators. From 2000 to 2005, Firms C and E had the highest use of 
cross-functional systems to ensure the effective implementation of their proactive internal energy efficiency initiatives. Firm E had the highest use of these systems during 2006-2007 due to the need to coordinate diverse cross-functional energy efficiency teams. Firms A and B experienced an identical degree of cross-functional MCS component use from 2000 to 2009, potentially due to both generators' high emission profiles and thus potential liabilities under the GCCPs. This led to a need to rely on cross-functional control systems to coordinate the monitoring and management of emissions and compliance carbon costs. Firms A and B also had the highest MCS use during the 2007-2009 period among the five generators, due to their more proactive carbon credit strategies to manage the potential carbon costs $(6.2 .5 ; 6.3 .5)$.

From October 2008, Firms A, B and E increased the use of inter-firm controls as they entered into relationships with external organisations to negotiate carbon credit purchases (e.g. 6.6.5). This reflects their changing strategic emphasis to focus on carbon credit strategies to meet their upcoming ETS compliance obligations. In contrast, crossfunctional MCS was used to a lower degree within Firm C, due to the cancellation of the carbon trading business and the substantial scale-back of internal energy efficiency initiatives as a result of internal restructuring from October 2008 (6.4.5). The other state-owned generators (B and E) also underwent internal restructuring, but as they had not established a carbon trading business, the impact of internal restructuring on their cross-functional systems was minimal. Figure 8-5 also illustrates that Firm D had the lowest use of cross-functional coordinating systems, corresponding to the limited scope of the internal energy efficiency initiatives it undertook. However, coordinating system use increased gradually to monitor the implementation of carbon trading activities to increase its retail competitiveness (6.5.4).

\section{Enabling controls and control systems}

Enabling controls and control systems are used by generators to increase employee innovation and managerial discretion and thus, to facilitate the development of the internal capabilities required to cope with external volatilities and pressures and build a GCCP-related competitive advantage. These MCS component types are primarily used in relation to generation, retail and carbon credit strategies.

In Firm C, the HRM system was used to train existing staff or recruit new employees who specialised in sustainability and carbon neutrality; the areas that reinforce its greenbrand retail strategy (6.4.3). In contrast, as part of its carbon credit strategy, Firm B employed dedicated carbon traders to build internal expertise to prepare for future ETS 
compliance obligations (6.3.4). These MCS component differences are consistent with the fact that Firm $\mathrm{C}$ has no carbon costs, while Firm B has high emission profile and thus substantial carbon costs (Table 7-1). Early on, Firm E also invested resources, e.g. through a separate budget, to promote customer energy efficiency and develop energy efficiency products and services, with a vision to make this one of its retail competitive advantages (6.6.2).

Furthermore, most generators (A, C, D, and E) used their HRM systems to acquire and develop staff expertise in a particular renewable technology so they could pursue a large-scale renewable development programme in the later periods. In Firms C and E, an overall budget for climate change initiatives was established to encourage managerial discretion and staff innovation in relation to projects and initiatives, that could result in a potential competitive advantage (6.4.4). Additionally, some generators (A, B, E) assigned a separate budget for carbon trading, either to develop staff skills in managing future carbon costs or to maximise potential revenue from carbon trading.

Figure 8-6: Use of enabling controls and control systems (2000-2009)

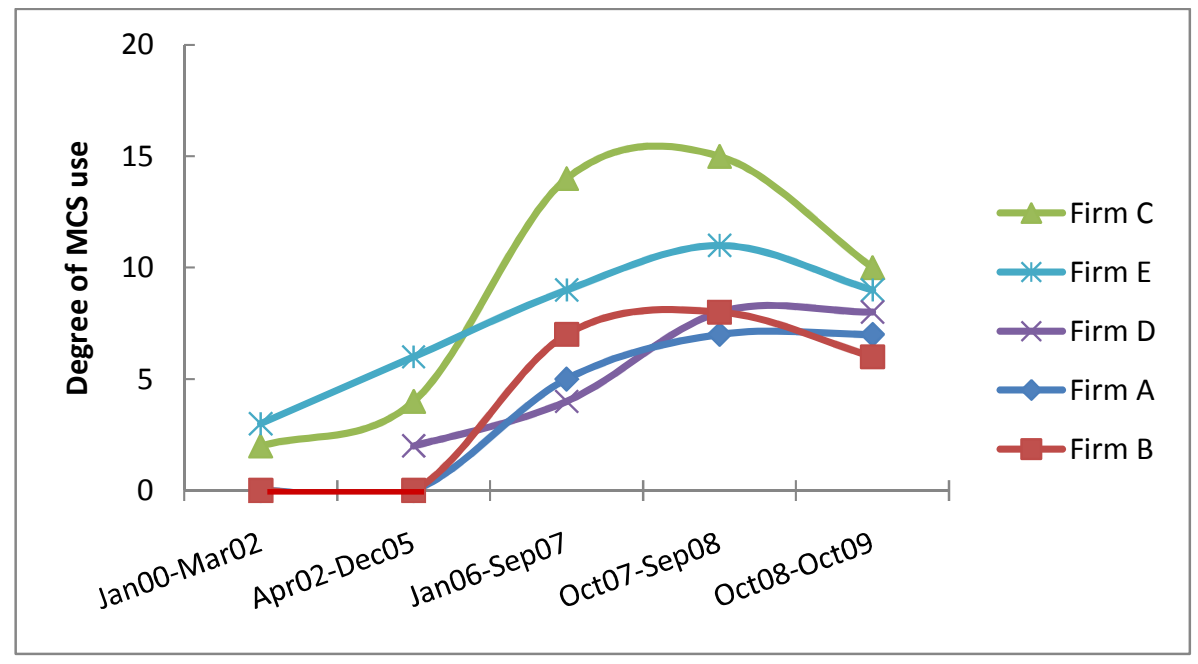

Figure 8-6 illustrates a general increase over time in the use of enabling control systems for all generators. However, there are substantial differences in the timing of the adoption of these systems and the degree of their use. In earlier periods, there was no enabling control system in the thermal-based Firms A and B. However, the renewable generators (C, D and E) began to use their MCS early on to develop renewable expertise. Firm E was the earliest and used its MCS to build internal expertise in customer energy efficiency. 
From January 2006 to September 2008, there has been a substantial increase in the use of enabling control systems in Firm C. Due to its decision to undertake proactive environmental strategies in response to the GCCPs (6.4.3), a separate budget was established and the HRM system was used to encourage innovation and expertise in wind development, carbon trading, and carbon neutrality. Firm E was behind Firm C, but had higher enabling control system use than other firms. This is because it established a budget for climate change initiatives and used the HRM system to develop geothermal expertise in this period. During 2006/07, Firm B recognised the need to develop carbon trading skills to prepare itself for future ETS compliance obligations. Therefore, it assigned a separate budget for carbon purchases and used the HR system to identify and recruit specialised carbon trading personnel (6.3.3). Firm A, initially behind Firm B in adopting these types of control systems, caught up and developed its geothermal human expertise and carbon trading capabilities to build a green advantage and manage its upcoming ETS compliance costs (6.2.3). Firm D had a lower use of enabling control systems than other four generators during Jan2006-Sep2007, due to its sole emphasis on the HRM system for wind energy expertise. However, it has since used a wider range of enabling control systems to develop the internal carbon trading skills required to implement differentiated carbon credit and retail strategies (6.5.3).

During the last period (2008/09), the use of enabling control systems within the stateowned generators (B, C, E) decreased. This is because governmental performance pressures led to a decrease in strategic emphasis and less resource discretion for developing expertise in carbon trading $(\mathrm{B}, \mathrm{C})$ and climate change initiatives $(\mathrm{C}, \mathrm{E})$ (Table 7-7). In contrast, because of capital market pressures, the publicly-listed Firms A and $\mathrm{D}$ considered expertise in carbon trading to be critical to their competitiveness in a GCCP-focused environment. Thus, the use of enabling control systems was maintained to support their environmental strategies.

\section{Risk monitoring systems}

As outlined in Table 8-2, there are also control systems that directly monitored changes in the external operating environments. Therefore, they are named as risk monitoring systems, with risk referring to the volatilities and fluctuations in the key institutional and economic environments of the generators. These risk monitoring systems provide externally-orientated information, based on which generators can make the appropriate strategic responses in order to maintain their competitiveness. The primary 
environmental strategies supported by risk monitoring systems include generation investment, retail, political and carbon credit strategies.

All the generators established a climate change risk register to identify, measure, and monitor a wide range of GCCP-related and climate change volatilities and pressures. By ranking each volatility or pressure by significance (potential impact) and time horizon (short, medium to long term), this risk register helped managers prioritise their attention and decision-making focus (e.g. 6.4.4). Additionally, the generators monitored market and regulatory changes such as movements in carbon markets, external fuel economics and modifications to the GCCPs (policy oversight system), to inform their carbon credit and generation strategies (e.g. 6.6.4). There were also systems that monitored competitors' GCCP-related competitive actions so the generators could adapt their retail strategies or undertake other appropriate responses. Understanding the political agenda of other organisations helped the generators to inform their political positions, e.g. to dismiss other generators' arguments in their GCCP submissions (e.g. 6.5.2). Generators also maintained a database to monitor changes in the demands and expectations of different customer groups. The database information was critical to generators in designing their retail strategies and developing new products and services (6.6.4).

Figure 8-7: Use of risk monitoring systems (2000-2009)

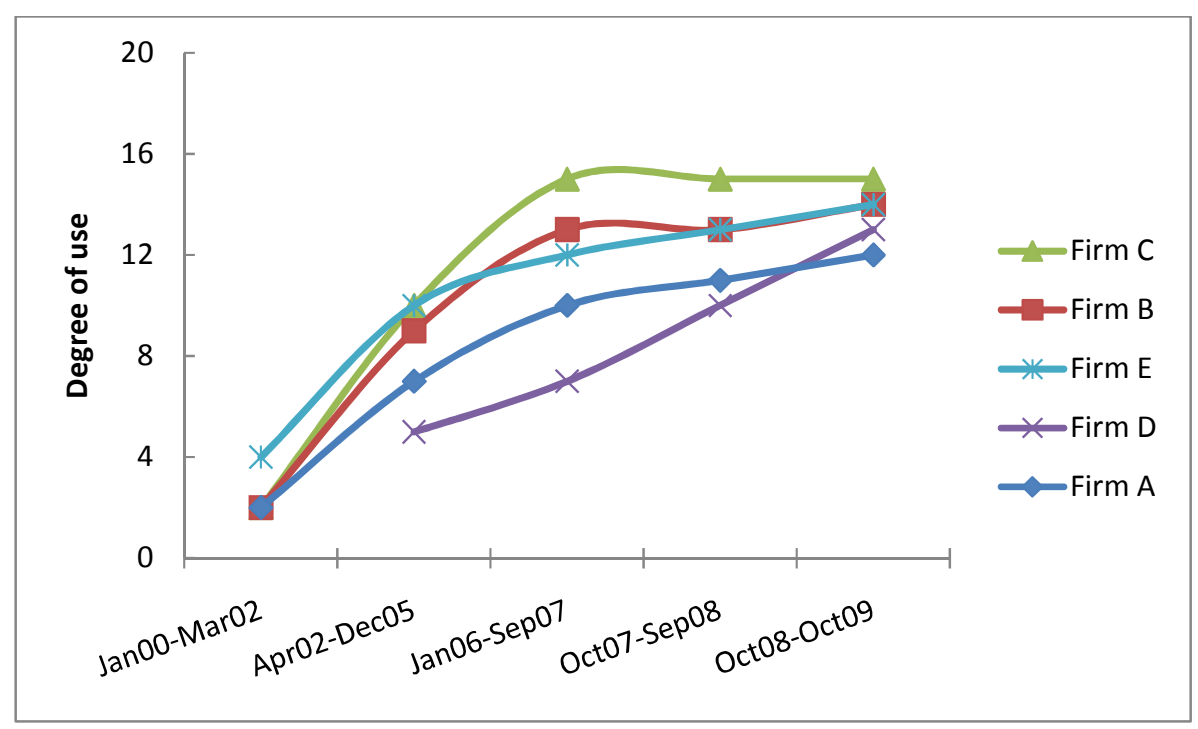

Figure 8-7 illustrates that the use of risk monitoring systems grew over time for all generators. This potentially reflects the increasingly complex external environments associated with the GCCPs and the resultant multiple volatilities and pressures to which the generators were exposed. Such complexity requires broader-scope monitoring systems and more attention to be placed on these systems at the strategic level. 
Interview data further suggests that these risk monitoring systems provided information to reduce the gap between organisational knowledge and changes in external environments, thus enabling more timely and effective organisational learning and adaptation.

As illustrated in Figure 8-7, Firm E led the industry during 2000-2005 period as it used its customer demand and expectation database to support its strategic emphasis on customer energy efficiency (6.6.2). In contrast, the other generators only had one or two risk monitoring systems and used them on an ad hoc basis. Since 2006, Firm C has had the highest use of risk monitoring systems in order to support the planning and implementation of different environmental strategies. Further, Firm B paid more attention to risk monitoring MCS components than Firm A (and Firm E during Jan2006 - Sep2007) because Firm B placed early emphasis on a carbon credit strategy. Firm A had lower risk monitoring use than Firms B and E because it did not pay as much attention to a carbon credit strategy (as Firm B) or a customer energy efficiency/retail strategy (as Firm E). Due to perceived low exposure to GCCP volatilities and pressures, Firm D was again behind the other generators in its use of risk monitoring systems before 2008. However, since October 2008, the change in its retail and carbon credit strategies has resulted in more strategic use of the carbon market monitoring system and customer demand and expectation database (6.5.4). By October 2009, the use of risk monitoring systems in Firms B, C and E had converged at a higher level than Firms A and D. This corresponds to the wider range of environmental strategies that received strategic emphasis within Firms B, C and E (Table 7-7), which led these generators to employ the information provided by risk monitoring systems more extensively during their strategic planning process.

\section{Legitimising controls and control systems}

Legitimising controls and control systems were used primarily to satisfy the demands and expectations of external stakeholders and thus maintain the generators' social legitimacy. The external disclosure of emission levels and sustainability reporting were undertaken by all the generators to implement their environmental policy and disclosure strategies and thereby demonstrate their environmental responsibility. Further, customer energy efficiency information systems were adopted to support customer energy efficiency strategy, driven by governmental pressures. The use of these systems did not add to the generators' profits or revenues and therefore did not result in significant changes at behavioural, processual or strategic levels. 
Other legitimising controls and control systems involved advice or inspection from independent verifiers or auditors and thus possibly changed generators' internal systems and processes. Such controls and control systems include: audits of AR sustainability content (A and B) and of emissions inventory (C); external awards or accreditation of organisational EMS (A and E); and the CarboNZero certification process (A, C, D). Generators undertook these control and control systems when implementing their environmental policy and carbon neutrality strategies, and to increase the transparency in emissions-related disclosure. By doing so, generators not only sought social acceptability, but also differentiated themselves from others and thus enhanced their market reputation.

Figure 8-8: Use of legitimising controls and control systems (2000-2009)

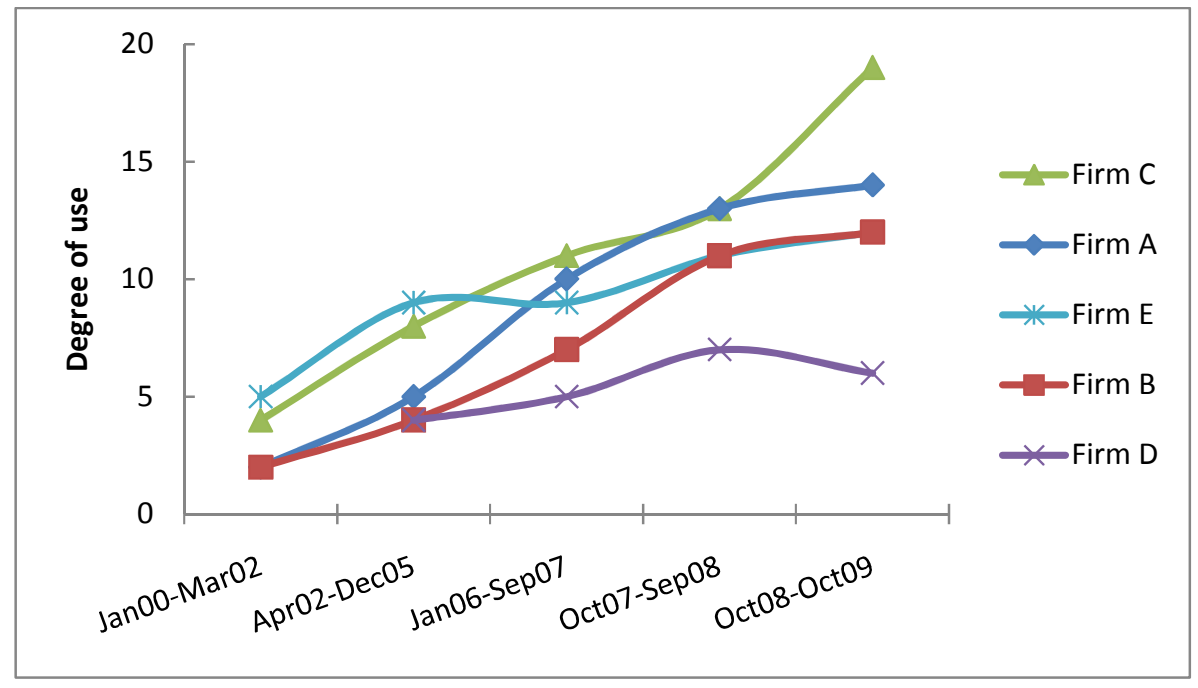

Figure 8-8 indicates that from January 2000 to September 2008, the use of legitimising controls and control systems increased to varying degrees for all five generators. This corresponds to the generators' recognition of increasing governmental and societal pressures regarding emissions reduction and sustainability. Firms $\mathrm{C}$ and $\mathrm{E}$ had the highest use of legitimising control systems before 2006. This is because they both adopted customer energy efficiency systems, sustainability reporting, emissions-related disclosure and gained accreditation for their EMSs, and used these controls extensively to build a reputation for high environmental performance. Since January 2006, Firm C's legitimising MCS use has surpassed Firm E. This is potentially due to Firm C's proactive renewable-focused environmental strategies (Figure 7-5), and thus a strong emphasis on legitimising MCS to improve its green brand. From October 2008, Firm C publicly issued a full disclosure of its emissions inventory and had this inventory and its sustainability report externally verified, thus reinforcing its strategic vision to 
differentiate and become a climate change leader. Thus, the use of Firm C's legitimising MCS components increased substantially and ranked the highest among the five generators by October 2009.

The rate of increase in Firm A's legitimising control system use was the highest among the five generators until October 2008. This is because it adopted multiple controls such as CarboNZero certification, sustainability reporting and the verification of sustainability content in ARs, to demonstrate its environmental responsibility and support its geothermal investment strategy. These legitimising controls are of strategic importance within Firm A since they supported its competitive strategy. In contrast, while Firm B perceived stronger societal pressures associated with its emissions profile, it did not have as many legitimising controls or place a strong emphasis on them. This tentatively suggests that Firm B was not as proactive in protecting its legitimacy despite its high societal exposure. Legitimising MCS component use in Firm D was substantially lower than the other generators, since Firm D paid little attention to emissions-related disclosure, customer energy efficiency, or carbon neutrality strategies (except for 2007/2008 when it pursued a carbon neutrality programme). During 20082009, legitimising MCS component use increased within Firms A, B, C and E as more strategic attention was paid to emissions-related disclosure strategies to manage social legitimacy in face of carbon-focused public pressures. In contrast, there is a drop in Firm D's legitimising MCS component use, caused by the cancellation of its carbon neutrality programme (6.5.4).

\subsubsection{MCS groups and their relationships to changes in environmental strategies}

Further analysis indicates that the eight MCS component types discussed in 8.2.1 and 8.2.2 above have a number of commonalities regarding their relationships with the environmental strategies and GCCP-related external and internal environments. Accordingly, these eight types of MCS components are classified into three component groups: internally-oriented strategy MCS; externally-oriented strategy MCS; and volatility/pressure-related MCS. These three groups are directly related to the three strategy objectives (Table 7-8).

To enable quantitative analysis, the degree of use for a particular MCS group was obtained by adding up the degrees of use across the component MCS types. This allows an explanation of the relationships between MCS design and use and changes in environmental strategies (P4). Each MCS component group is discussed next. 


\section{Group one: Internally-oriented strategy MCS for cost control}

Belief systems, boundary controls, diagnostic controls and control systems and crossfunctional controls and coordinating systems were grouped into internally-oriented strategy MCS. This is because these four are primarily designed and used to support changes in emissions management, internal energy efficiency, internal restructuring, generation and carbon credit strategies which are driven by cost control concerns.

Figure 8-9: Use of internally-orientated strategy MCS component group (20002009)

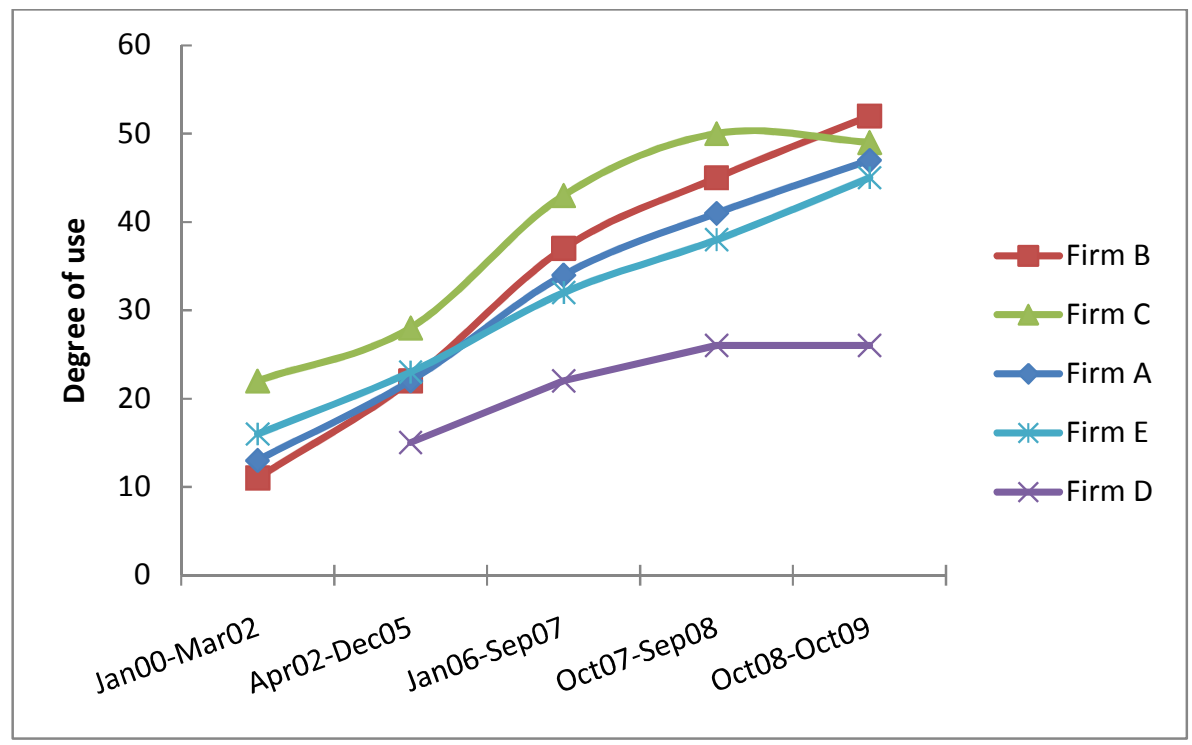

As shown in Figure 8-9, the degree of use of internally-oriented strategy MCS component group increased consistently over time. This is potentially explained by the rising complexity of generators' environmental strategies and the wider functional involvement required for their implementation (7.5.1). Firms A, B and E display a similar degree of use of these internally-oriented strategy MCS components. This similarity is probably associated with the generation emissions profile and the carbon obligations these generators have under the GCCPs, and the need to focus internal MCS efforts on emissions management, carbon credit and generation investment strategies for carbon cost control. Consistent with the carbon costs of each organisation, the difference in the rate of change in MCS use between these three generators correlates to the variations in emissions profile, with highest-emitting firm (B) experiencing the fastest growth in its MCS use. Further, the degree of use of these internally-oriented strategy MCS was highest in Firm C due to its renewable-only generation strategy and the extensive and wide-scope internal energy efficiency initiatives adopted. The simultaneous execution of these initiatives required a strong supporting belief system, 
regular monitoring and reporting, strong internal commitment and coordination and clear guidance and responsibility assignment. The slight reduction in MCS use during Oct2008-Oct2009 is probably related to the decreased scope of its internal energy efficiency strategy due to Government performance pressures. Firm D ranked the lowest in internally-orientated strategy MCS component use in all periods, because it designed and used the smallest number of internally-oriented strategy controls and control systems. This corresponds to the narrower scope of Firm D's energy efficiency efforts, which is potentially associated with its smaller size.

\section{Group two: Externally-oriented strategy MCS for competitiveness}

Interactive control systems, enabling control systems and risk monitoring systems are grouped into externally-oriented strategy MCS components for competitiveness. This is because these three control systems tend to be used to implement changes in the environmental strategies aimed at gaining a competitive advantage or helping generators to respond effectively to a key external volatility or pressure. These strategies include generation, political, retail, and carbon credit strategies as discussed in Chapter 7 (7.5, Table 7-8). Additionally, a number of externally-oriented control systems were used to support a customer energy efficiency strategy, since this strategy is integrated into some generators' retail strategy.

Figure 8-10: Use of externally-orientated strategy MCS component group (20002009)

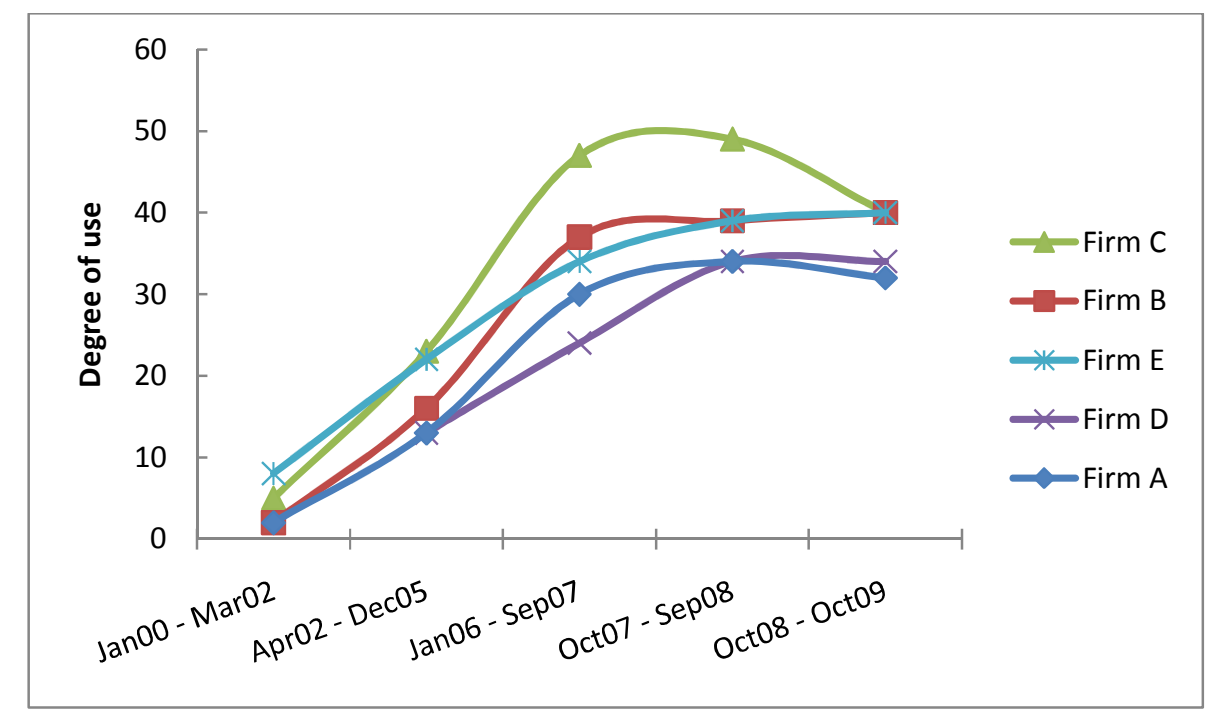

Figure 8-10 shows that from 2000 to 2008, the use of externally-oriented strategy MCS components increased for all generators, potentially due to the rising level of strategy proactiveness (Figure 7-5). Firm E had the highest use of externally-oriented strategy MCS from January 2002 to December 2005 as it used this MCS component group to 
monitor customer demand and develop internal expertise in customer energy efficiency as part of its proactive retail strategy. This is probably due to Firm E's low carbon costs (Table 7-1) and internal expertise in customer energy efficiency, and thus increasing strategic emphasis on customer-based revenue management. From January 2006 to September 2008, Firm C had the highest use of this MCS component group. Its high level of strategy proactiveness (Figure 7-5) required the use of externally-oriented strategy MCS components to monitor external environmental changes and enable employee empowerment to support strategy planning and implementation. Further, thermal-based Firms A and B had similar increases in their externally-oriented strategy MCS component use, primarily due to the utilisation of risk monitoring and interactive control systems to support the planning of their generation strategies and carbon credit strategies. The change in this MCS component group use within renewable-based Firms $\mathrm{D}$ and $\mathrm{E}$ was similar, potentially associated with increasing use of enabling and interactive control systems to implement their green-focused generation and retail strategies.

From 2008 to 2009, the use of this MCS component group decreased in Firms A and C. Both generators employed their MCS less interactively, consistent with their reduced strategy proactiveness due to regulatory uncertainty and the less positive incentive for renewable investments (7.5). Additionally, Firm $\mathrm{C}$ experienced a sharp decline in the use of enabling MCS due to the government financial pressures and thus reduced the scope and strategic emphasis given to its carbon credit strategy (6.4.5). Firms B and E were exposed to similar pressures from the Government and also used these enabling MCS components less. However, the strategy review undertaken in $\mathrm{B}$ and $\mathrm{E}$ simultaneously required higher interactive MCS use and more extensive employment of risk monitoring systems to facilitate the strategy renewal process (6.3.5 and 6.6.5). Consequently, the total use of externally-oriented strategy MCS components slightly increased within Firms B and E during 2008-2009. Similar to Firms A and C, Firm D experienced a drop in interactive MCS use associated with reactive political and generation strategies (7.5). However, it employed information from risk monitoring systems more extensively to support its differentiation carbon credit and retail strategies. This resulted in the stabilisation of externally-oriented strategy MCS component use within Firm D. 


\section{Group three: Legitimising MCS for legitimacy}

The remaining MCS component type, legitimising controls and control systems, respond directly to the demands and pressures in the generators' external institutional environments. As discussed in 8.2.2, these controls and control systems are undertaken to implement changes in environmental policy, customer energy efficiency, emissionsrelated disclosure and carbon neutrality strategies, which are in turn driven by legitimacy needs (Table 7-8). Accordingly, most generators increased the design and use of legitimising MCS over time in response to increasingly complex GCCP-related institutional environments, and the degree of use varied across the generators in accordance with the level of strategic emphasis and the degree of strategy proactiveness pursued within each time period.

\section{Change in the use of different MCS groups over time}

The use of each MCS component type, examined in Sections 8.2.1 and 8.2.2, is averaged across the five generators within each time period. Next, this resulted degree of use is averaged across the MCS component types comprising a MCS component group in order to gain an averaged degree of use for each MCS component group. This enables an assessment to be made about the trend of change over time for each MCS component group and inter-group variations. Figure 8-11 illustrates changes in the average use of the three MCS groups over time.

Figure 8-11: Averaged degree of MCS component group use (2000-2009)

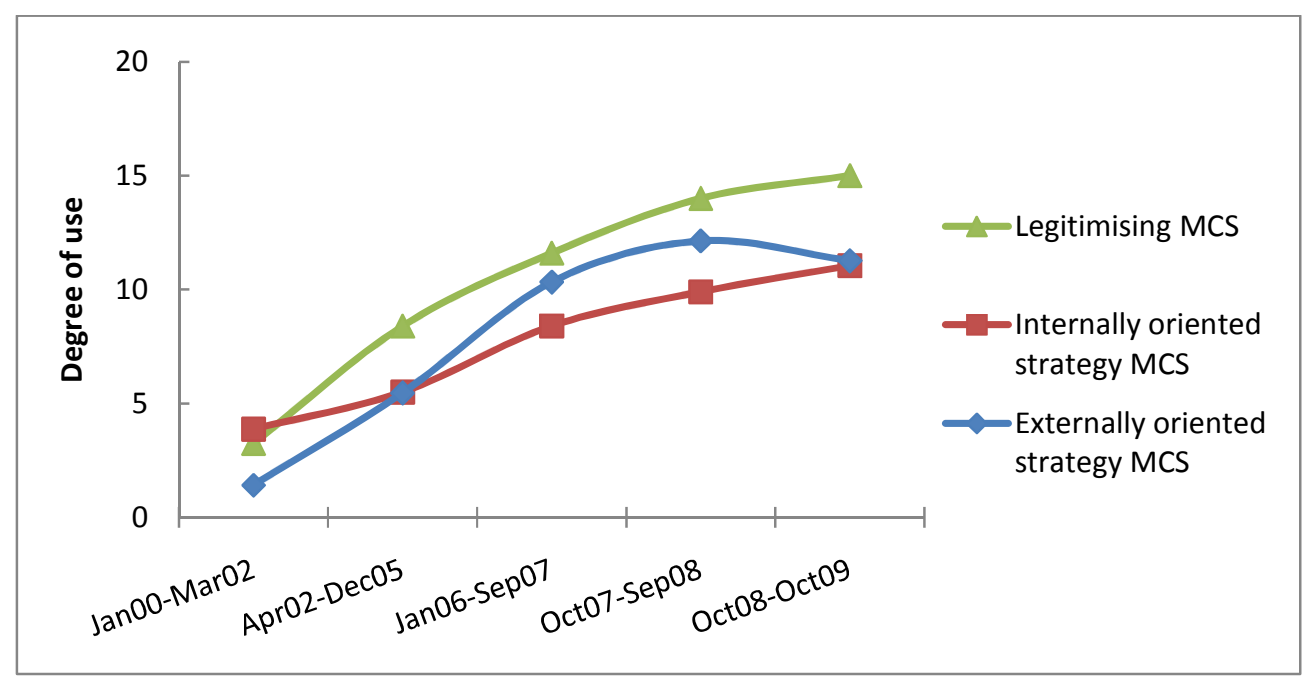

Figure 8-11 illustrates that the use of internally-orientated strategy MCS component group increased gradually from 2000 to 2009. Over time, the generators perceived high carbon costs associated with generation emissions and operational and transactional costs associated with non-generation emissions. This led them to place increasing 
strategic emphasis on emissions management, generation investment, and carbon credit strategies (for thermal generators) and internal energy efficiency strategies (for renewable generators), which had a cost control focus (Tables 7-7 and 7-8). An increasing number of diagnostic controls and controls systems were employed and they received more emphasis at top management levels since they were critical to the achievement of these cost-focused environmental strategies. Additionally, there is a need for stronger internal coordination between different functions, departments and levels of management to minimise transactional costs (cross-functional MCS components). Belief systems and boundary controls were also used to increase cost consciousness and to prevent internal opportunism and inefficiencies in strategy implementation. Consequently, the combined use of these four MCS component types resulted in an increasing degree of internally-oriented MCS component group use.

The use of externally-oriented strategy MCS increased from Jan2000 to Sep2008, but has reduced since. As discussed in Chapter 7 (Figure 7-5), from 2000 to 2008, the generators increased the proactiveness of some environmental strategies, including generation investment and retail strategies, to protect or enhance their competitiveness. Therefore, the generators used their MCS components more interactively and in an enabling manner (interactive and enabling MCS components) to encourage strategic renewal and the development of internal skills and expertise required to implement competitiveness-driven strategies. The generators also used the information provided by risk monitoring systems more extensively to inform the review of existing strategies and the formulation of new ones. In contrast, from 2008 to 2009, due to regulatory uncertainty and potential ETS changes, most generators reduced their strategy proactiveness, especially in relation to political and generation strategies. This in turn required less input from lower management levels into the strategy planning process and lessened the importance of building renewable expertise, thus leading to a decrease in the use of interactive and enabling control systems. Additionally, governmental performance pressures also led state-owned generators to reduce their strategic emphasis on carbon trading and to refocus on core businesses. This reduced the use of the enabling control systems that were previously utilised for the development of carbon-related expertise. In combination, these factors resulted in a drop in externallyoriented strategy MCS use from October 2008 to October 2009.

Figure 8-11 also indicates that the use of legitimising MCS components increased at a consistent rate throughout the period. As the generators recognised the increasing 
governmental and societal pressures related to carbon emissions, they undertook more proactive environmental strategies in response (customer energy efficiency, external disclosure, environmental policy, and carbon neutrality strategies). Consequently, to implement these strategies, they adopted customer energy efficiency information systems, and undertook more extensive emissions disclosure and verification and widerscope carbon neutrality processes (legitimising MCS components). They also paid more attention to these controls at the top management levels as they realised that responding to institutional pressures is a prerequisite for social acceptability and thus organisational survival in the long term. For some generators, legitimising MCS components received an increasingly high level of strategic emphasis because its adoption was believed to increase the organisation' legitimacy and thus promote a green advantage in a GCCPfocused context. Additionally, the higher level of legitimising MCS component use than externally-oriented and internally-oriented strategy MCS component use confirms the importance of legitimising MCS components from a top management perspective.

Overall, this section (8.2) has outlined the changes in the design and use of organisational MCS components to support the eleven environmental strategies undertaken by the generators as discussed in Chapter 7. Eight types of controls and control systems were found, four of which supported Simons (1991) MCS categories and the other four were new and have not been examined adequately in the prior literature. These eight MCS component types were used at varying degrees across generators and time periods, depending on the degree of strategy proactiveness and the level of strategic emphasis of their environmental strategies. Further, they can be classified into three main groups: internally-oriented strategy MCS components for cost control; externally-oriented strategy MCS components for competitiveness; and legitimising MCS components for legitimacy. In suggesting that changes in environmental strategies require corresponding modifications in the design, use, and objectives of MCS, these results provide empirical support for P4. Furthermore, they indicate that organisational MCS components were used not only to achieve an objective of internal efficiency but also to support the pursuit and development of a competitive advantage, and to ensure and enhance the generators' social legitimacy in a carbon-constrained context (P4). Understanding these different objectives is important because it provides insights into the motivations underlying the design and use of different MCS component types and groups. The next section discusses in more detail the MCS objectives. 


\subsection{MCS objectives}

This section analyses the objectives suggested above in more detail and examines any additional ones that potentially motivated the design and use of organisational MCS components (P4). The analysis finds commonalities relating to these MCS objectives, but also identifies differences in their distribution across the generators. The next section discusses the common objectives that were found across the five generators in their MCS design and use.

\subsubsection{Common objectives of organisational MCS}

Analysis conducted into the objectives underlying all the controls and control systems undertaken by the five generators suggests five common MCS objectives: 1) reducing compliance carbon costs, 2) reducing transactional costs, 3) reducing operating overhead costs, 4) enhancing/protect market competitiveness, and 5) achieving legitimacy. 
Table 8-3 summarises and lists these objectives and matches each organisational objective with the appropriate MCS component types and groups (as examined in Section 8.2) and the environmental strategic changes driving their design and use. This table suggests that different MCS component groups were aimed primarily at different objectives: internally-oriented strategy MCS components for cost reduction (carbon, operating or transactional), externally-oriented strategy MCS components for competitiveness, and legitimising MCS components for legitimacy. Each objective is discussed next. 
Table 8-3: Ranking of organisational objectives of MCS, matching with MCS component types and groups and environmental strategies

\begin{tabular}{|c|c|c|c|}
\hline MCS objectives & Correspond to MCS type & Part of MCS group & $\begin{array}{l}\text { Supporting changes in } \\
\text { environmental strategy }\end{array}$ \\
\hline $\begin{array}{l}\text { 1. Reduce } \\
\text { compliance } \\
\text { carbon costs }\end{array}$ & $\begin{array}{l}\text { Diagnostic controls and } \\
\text { control systems } \\
\text { Belief systems }\end{array}$ & $\begin{array}{l}\text { Internally-oriented } \\
\text { strategy MCS }\end{array}$ & $\begin{array}{l}\text { Emissions management } \\
\text { strategy } \\
\text { Carbon credit strategy } \\
\text { Generation strategy }\end{array}$ \\
\hline $\begin{array}{l}\text { 2. Reduce operating } \\
\text { overhead cost }\end{array}$ & $\begin{array}{l}\text { Diagnostic controls and } \\
\text { control systems } \\
\text { Belief systems }\end{array}$ & $\begin{array}{l}\text { Internally-oriented } \\
\text { strategy MCS }\end{array}$ & $\begin{array}{l}\text { Internal energy efficiency } \\
\text { strategy } \\
\text { Carbon neutrality strategy } \\
\text { Internal restructuring } \\
\end{array}$ \\
\hline $\begin{array}{l}\text { 3. Reduce } \\
\text { transactional cost }\end{array}$ & $\begin{array}{l}\text { Cross-functional } \\
\text { coordinating systems } \\
\text { Boundary control } \\
\text { systems }\end{array}$ & $\begin{array}{l}\text { Internally-oriented } \\
\text { strategy MCS }\end{array}$ & $\begin{array}{l}\text { Emissions management } \\
\text { strategy } \\
\text { Internal energy efficiency } \\
\text { strategy } \\
\text { Carbon credit strategy } \\
\text { Environmental policy }\end{array}$ \\
\hline $\begin{array}{l}\text { 4. Enhance/Protect } \\
\text { market } \\
\text { competitiveness }\end{array}$ & $\begin{array}{l}\text { Interactive control } \\
\text { systems } \\
\text { Enabling control systems } \\
\text { Risk monitoring systems } \\
\text { Belief systems }\end{array}$ & $\begin{array}{l}\text { Externally- } \\
\text { oriented strategy } \\
\text { MCS }\end{array}$ & $\begin{array}{l}\text { Generation strategy } \\
\text { Carbon credit strategy } \\
\text { Retail strategy } \\
\text { Political strategy } \\
\text { Carbon neutrality strategy } \\
\text { Customer energy efficiency } \\
\text { strategy }\end{array}$ \\
\hline $\begin{array}{l}\text { 5. Achieve } \\
\text { legitimacy }\end{array}$ & $\begin{array}{l}\text { Legitimising controls and } \\
\text { control systems }\end{array}$ & Legitimising MCS & $\begin{array}{l}\text { Emissions-related disclosure } \\
\text { strategy } \\
\text { Carbon neutrality strategy } \\
\text { Environmental policy } \\
\text { Customer energy efficiency } \\
\text { strategy }\end{array}$ \\
\hline
\end{tabular}

\section{Reducing compliance carbon costs}

Many controls and control systems were designed and used within thermal generators to reduce the compliance carbon costs as part of their emissions management, carbon credit, and generation strategies. Diagnostic controls and control systems such as emissions monitoring system and reduction targets (Table 8-1) were used to ensure effective reduction of generation carbon missions and thus lower carbon costs. Similarly, carbon-related budgetary controls optimised the cost control when buying and selling carbon credits for ETS compliance purposes. The monitoring of emissions measures also enabled the generators to make a strategic shift to renewable investments (A, B, and E). In some generators, a belief system such as a financially-focused organisational culture supplemented diagnostic controls by creating an internal environment which expected and rewarded high cost awareness and control, thus increasing the effectiveness of managing compliance carbon costs. Belief systems led to stronger cost control within carbon trading function of Firm A and supported changes in 
generation investment strategies within Firm B (to focus on making an adequate return from 2008) and Firm E (renewable-focused generation investment from 2007). Thus, the design and use of diagnostic controls and belief systems within these generators support a TCE-based objective and P4.

\section{Reducing operating overhead costs}

For most generators, reducing operating overhead costs associated with non-generation emissions was an important MCS objective. This is because the activities giving rise to such emissions (electricity, staff travel and paper use) caused significant overhead costs, which needed to be managed as part of internal cost control. An internal energy efficiency strategy was undertaken with a primary purpose to reduce these costs while the adoption of a carbon neutrality strategy also led to a reduction in non-generation emissions and associated overhead costs. To ensure the implementation of these strategies, these generators adopted many controls and control systems diagnostically (e.g. non-generation emissions measures and reduction targets and the link of the emissions measures to managerial performance evaluation) and environmentallyfocused belief systems. Furthermore, from October 2008, under governmental pressures, state-owned generators tightened budgetary controls as part of their internal restructuring, aimed at reducing operating costs and hence, improving internal efficiency. Consequently, this use of diagnostic controls and control systems and belief systems is consistent with P4 and a TCE-based explanation.

\section{Reducing transactional costs}

Reducing transactional costs was the primary objective for the use of cross-functional controls and coordinating systems and boundary controls. Cross-functional controls and control systems enhanced internal monitoring and coordination when implementing emissions management and internal energy efficiency strategies and ensured interorganisational cooperation in carbon credit strategy. Further, boundary controls (e.g. carbon-related spending limits and the separation of duties) minimised opportunism in carbon trading transactions (carbon credit strategy) and aligned employee behaviour with organisational environmental policy (goal congruence). By improving coordination and reducing opportunism, these MCS component types contributed to a reduction in transactional costs and thus ensured internal efficiency in respect of environmental strategies. Consequently, the focus on transactional cost economising supports $\mathrm{P} 4$ and a TCE view. 


\section{Protecting/ Enhancing competitiveness}

Enhancing or protecting generators' competitiveness was the primary objective underlying the design and use of interactive control systems, enabling controls and control systems, as well as risk monitoring systems. Interactive control systems were used extensively within both renewable and thermal generators during the strategic planning process to revise their environmental strategies in response to GCCP changes and associated volatilities and pressures. Most generators used MCS components, such as an emission monitoring system and carbon price monitoring system, in an interactive manner to generate internal debate about the choice of a generation investment strategy that could maintain or enhance market competitiveness. Some generators also used internal communication and profit planning systems interactively to encourage creative carbon trading and retail initiatives that could be used to respond effectively to the GCCPs, and thus protect competitive advantage. Additionally, the generators used accounting-based analysis interactively within their political strategy to influence the policy-makers and regulators and their GCCP designs. Interview data suggest that such accounting use was not driven by a potential to increase political power, but primarily a need to protect or enhance the organisation's existing competitiveness.

Enabling controls and control systems (e.g. HRM system) were employed within the renewable generators to develop staff expertise in a particular renewable technology and thus, support their differentiation renewable-based generation strategy. As part of their carbon credit and retail strategies, some generators also established appropriate budgets to allocate resources for internal capability development in carbon trading, carbon neutrality, or customer energy efficiency. This would enable them to gain a competitive advantage in a future carbon-constrained economy.

The generators also designed and used risk monitoring systems with a competitivenessdriven objective. This MCS component type monitored changes in GCCP-related economic and institutional environments and provided information that allowed managers to make strategic assessment and adapt to external changes. The information from the policy oversight system, external fuel economics monitoring system and carbon market monitoring system enables the evaluation and formulation of generation investment and carbon credit strategies. The competitor actions monitoring system and customer demand and expectation database allowed the planning of retail strategy. Additionally, the policy oversight system and external organisations' political agenda monitoring systems provided information for generators to choose an appropriate 
political strategy. Therefore, the design and use of risk monitoring systems were driven by a need to continually assess the fitness of current environmental strategies against the changing external environments and formulate new ones, if necessary in order to preserve or enhance the generators' competitiveness. Additionally, belief systems were used within Firm $\mathrm{C}$ to support its renewable-only generation investment strategy and thus, contribute to its green-focused market competitiveness.

Therefore, these four MCS component types are used to enable generators to develop internal capabilities and to review and renew their environmental strategies in an informed and timely manner so that their market competitiveness is preserved or enhanced. Therefore, these results support P4 and a RBT-based view of organisational MCS.

\section{Achieving social legitimacy}

Additionally, most generators sought to achieve social legitimacy through their legitimising MCS components. This MCS component was used by generators to demonstrate publicly that they conformed to external GCCP-related institutional pressures and expectations. Their adoption is thus driven by legitimacy-driven environmental strategies including emissions-related disclosure and carbon neutrality strategies, environmental policy and customer energy efficiency strategy. Some generators sought to legitimate themselves by using controls and control systems that involved verification by an external organisation which potentially lead to internal changes. This is the case with the external audit of emissions inventory, carbon neutrality certification system and external awards for EMS. In contrast, other legitimising controls, such as emissions-related disclosure, sustainability reporting and customer energy efficiency information systems, provided general statements to external stakeholders without entailing major internal behavioural and processual changes. Hence, the use of these controls and control systems is consistent with an ITbased view that generators employ organisational MCS components to ensure social legitimacy.

Overall, the results indicate that all five generators designed and used their MCS to implement different environmental strategies and achieve three different objectives simultaneously (competitiveness, cost control, and legitimacy) (P4). This provides empirical support for the simultaneous use of RBT, TCE and IT to explain the objectives driving a generator's organisational MCS. Accordingly, the design and use of externally-oriented strategy MCS components is explained from a RBT competitiveness 
perspective, while TCE explains the motivations for an internally-oriented cost strategy MCS components and an IT-based view is appropriate for examining legitimising MCS components. Further, each MCS objective was driven by the primary objective of change in particular environmental strategies, thus providing further support for P4. However, these different objectives did not receive equal importance within the five generators, as discussed in the next section.

\subsubsection{Distribution of MCS objectives}

Figure 8-12 demonstrates the distribution of the different objectives of organisational MCS across the five generators, based on the data from October 2007 to October 2009. This time period is the most relevant as it was when there were plans for and suggested changes to an ETS which led to the highest degree of external GCCP exposure (Figure 7-1). Thus, analysing MCS objectives in this period captures the highest number of controls and control systems employed by the generators and their associated objective diversity. To enable this analysis, the primary objective motivating each control and control system highlighted in Tables 8-1 and 8-2 was determined. This step was undertaken for all controls and control systems constituting the organisational MCS of each generator until all the objectives underlying the five generators' MCSs were attained. The number of controls and control systems driven by different objectives was then divided by the total sum of controls and control systems within each generator to obtain the relative importance (in terms of \%).

Figure 8-12: Distribution of MCS objectives (2007-2009)

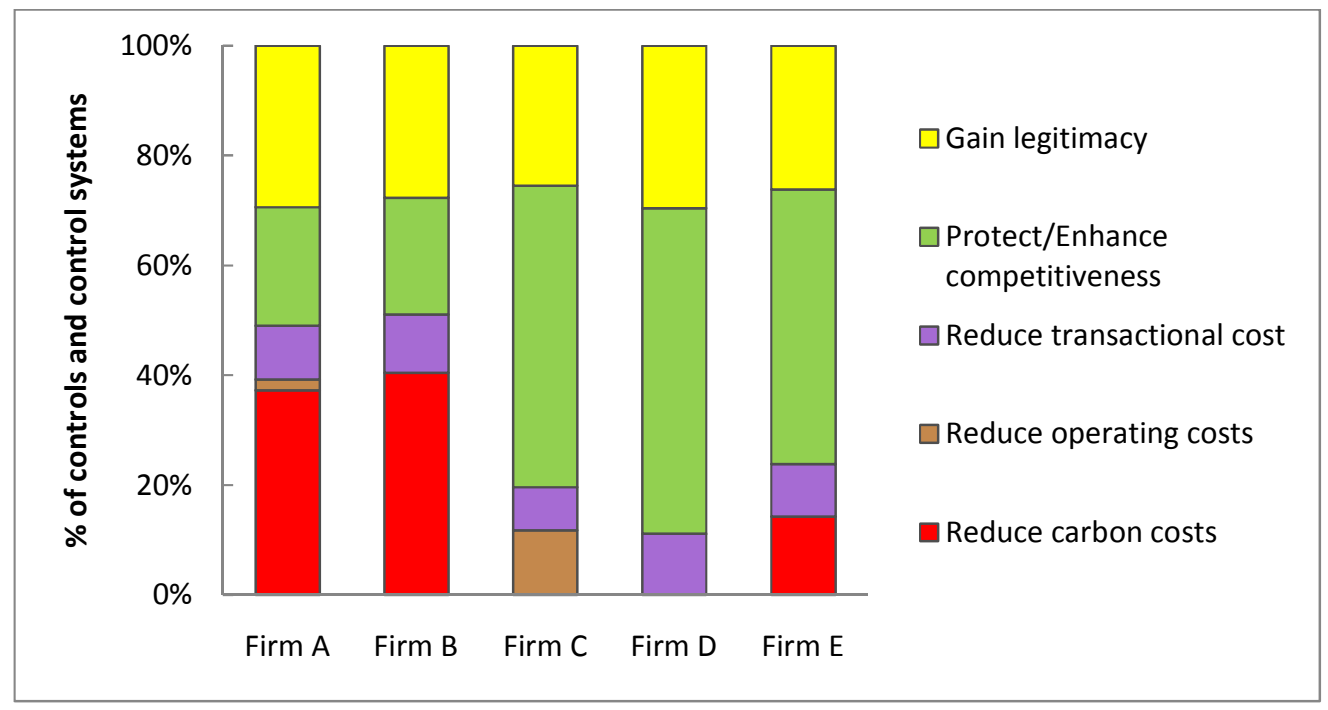

Figure 8-12 demonstrates significant differences in the focus of the objectives intended by each generator in establishing and using their organisational MCS. These inter-firm 
differences were consistent with inter-firm differences observed within the objectives motivating the environmental strategic changes discussed in Chapter 7 (Table 7-9). Based on Figure 8-12, Table 8-4 ranks the MCS objectives by the frequency with which they underlie a generator's controls and control systems. Accordingly, the ranking of all MCS objectives, within the five generators, is identical to that of the strategy objectives (Table 7-9).

Table 8-4: Ranking of MCS objectives in the five generators (2007-2009)

\begin{tabular}{|c|c|c|c|c|c|}
\hline $\begin{array}{l}\text { Ranking by } \\
\text { frequency }\end{array}$ & Firm A & Firm B & Firm C & Firm D & Firm E \\
\hline 1 & $\begin{array}{l}\text { Reduce carbon } \\
\text { cost }\end{array}$ & $\begin{array}{l}\text { Reduce carbon } \\
\text { cost }\end{array}$ & $\begin{array}{l}\text { Protect/Enhance } \\
\text { competitiveness }\end{array}$ & $\begin{array}{l}\text { Protect/Enhance } \\
\text { competitiveness }\end{array}$ & $\begin{array}{l}\text { Protect/Enhance } \\
\text { competitiveness }\end{array}$ \\
\hline 2 & Gain legitimacy & Gain legitimacy & Gain legitimacy & Gain legitimacy & Gain legitimacy \\
\hline 3 & $\begin{array}{l}\text { Protect/Enhance } \\
\text { competitiveness }\end{array}$ & $\begin{array}{l}\text { Protect/Enhance } \\
\text { competitiveness }\end{array}$ & $\begin{array}{c}\text { Reduce } \\
\text { operational cost }\end{array}$ & $\begin{array}{c}\text { Reduce } \\
\text { transactional cost }\end{array}$ & $\begin{array}{l}\text { Reduce carbon } \\
\text { cost }\end{array}$ \\
\hline 4 & $\begin{array}{c}\text { Reduce } \\
\text { transactional cost }\end{array}$ & $\begin{array}{c}\text { Reduce } \\
\text { transactional cost }\end{array}$ & $\begin{array}{c}\text { Reduce } \\
\text { transactional cost }\end{array}$ & & $\begin{array}{c}\text { Reduce } \\
\text { transactional cost }\end{array}$ \\
\hline
\end{tabular}

Thermal generators (Firms A and B) used their MCS to primarily reduce the carbon costs associated with fulfilling their ETS compliance obligations. This is consistent with the strategic emphasis placed on emissions management and carbon credit strategies and the primary objective of their environmental strategies which was carbon cost control (Tables 7-7 and 7-8). Managing social legitimacy is the second most important strategic and MCS objective, due to their high carbon emissions profile and associated institutional pressures. This corresponds to the strategic emphasis placed on their emissions-related disclosure strategies. Additionally, competitiveness is also a MCS concern in Firms A and B, since the GCCPs have the potential to change their competitiveness significantly. Organisational MCS was used in Firm A to support the development of geothermal expertise and a competitive advantage due to implementing its differentiated generation strategy. In contrast, Firm B used its MCS interactively in its GCCP-related investment planning and political processes, in order to protect its thermal competitiveness.

In the renewable generators (Firms C, D and E), competitiveness is the key concern of MCS design and use. Such focus in MCS objectives ensures their controls and control systems were used to support renewable generation investment strategies and green branding retail strategies. They also used accounting-based analysis in their political strategy to support the proposed GCCPs and thus enhance their renewable-related advantage. A competitiveness-focused MCS corresponds to the strategic emphasis placed on the generation, retail, and political strategies within these generators (Table 7- 
7). Renewable generators also had an interest to use their MCS to manage and disclose their non-generation emissions to show their climate change responsibility. This is consistent with the strategic emphasis placed on carbon neutrality and emissions-related disclosure strategies which aims to maintain legitimacy. A MCS and strategic focus on legitimating these generators to internal and external stakeholders also enabled them to reinforce their green brand.

Additionally, due to rapid organisational growth, Firm $\mathrm{C}$ experienced a high level of non-generation emissions and associated operating costs. Consequently, it established a proactive internal energy efficiency strategy and used MCS to measure, monitor, and control non-generation emissions, and thus reduce operating costs. Further, while also having an ETS compliance obligation, Firm E's emissions profile is significantly smaller than Firms A and B and thus, carbon cost control was considered only a secondary MCS objective.

The above table also suggests that reducing transactional cost as a MCS objective was only of secondary importance across the five generators. The use of boundary and crossfunctional MCS enables emissions management, internal energy efficiency and carbon credit strategies and environmental policy to be implemented with the least transactional costs and thus, optimise internal efficiency.

The analysis further suggests that the objectives of MCS design and use were consistent with the objectives underlying the choice of environmental strategies in each generator. Further, a strong focus on particular MCS objectives is driven by the strategic emphasis placed on particular environmental strategies by management. Further, this provides support for P4.

\subsection{Summary}

To summarise, this chapter has investigated the design, use, and objectives of organisational MCS employed to support changes in generators' GCCP-related environmental strategies, as presented in Chapter 7. It was found that each generator's MCS incorporates a range of controls and control systems which can be classified into distinct MCS component types and groups. In addition to the boundary controls, belief systems, diagnostic and interactive control systems as suggested by Simons (1991), generators adopted additional MCS component types: enabling controls and control systems; cross-functional controls and coordinating systems; risk monitoring systems; and legitimising controls and systems. While a generator may establish and use all these 
MCS component types, their degree of use and manner of use (diagnostic or interactive) varied across generators and over time, governed by the level of proactiveness and the relative priority (emphasis) of the environmental strategies that they supported. Further, these eight MCS component types can be grouped in three MCS component groups, each of which served a different MCS objective: i) internally-oriented strategy MCS for cost control, ii) externally-oriented strategy MCS for enhancing and protecting competitiveness, and iii) legitimising MCS for gaining and maintaining social legitimacy. While pursuing common MCS objectives, the importance of each objective varies across the generators depending on the primary objectives and strategic emphasis placed on each generator's environmental strategies. Overall, depending on the MCS component type and group examined, the results provide empirical support for the use of RBT, TCE or IT in this study. Proposition 4 is also supported as these results suggest that a change in environmental strategies has significant impacts on organisational MCS design, use and objectives.

The next chapter, Chapter 9, discusses the Chapter 6 evidence and analysis from Chapters 7 and 8 , in relation to the research question, the proposed research framework and the four propositions (P1, P2, P3, and P4). This provides an understanding of this study's theoretical and practical contributions to the literature. Chapter 9 also outlines the limitations of this study and opportunities for future research. 


\section{Chapter 9: Discussion and Conclusion}

\subsection{Introduction}

The GCCPs planned and modified by New Zealand Government from 2002 to 2009, have the potential to impose significant carbon-related costs on generators and increase the volatilities and pressures within the external environments in which generators operate. The key question arises as to what organisational changes have been undertaken within these generators to enable them to reduce these costs and prepare for the changing environments. This study's research question is therefore:

What are the MCS implications arising from strategic choices New Zealand electricity generators make in managing economic volatility and societal pressures resulting from a prolonged period of GCCP changes?

To answer this research question, a multiple-theory research framework was developed based on three theories, TCE, IT, and RBT and the prior literature. Four associated propositions were given in Chapter 4 and are restated below (9.2.2). In conducting the multiple-case study of the five generators in the NZEI (a discussed in Chapter 5), evidence related to environmental strategies and MCS change within each generator from January 2000 to October 2009 was provided in Chapter 6. The results of analysing this evidence were then presented in the two subsequent chapters. Chapter 7 identified the key GCCP-related external volatilities and pressures and internal characteristics and capabilities, discussed how they influenced generators' environmental strategies, and explained why different generators made different strategic changes in response to the GCCPs. Chapter 8 examined how MCS design, use and objectives were modified over time to support these environmental strategic changes.

This chapter concludes this study by firstly, discussing the implications for organisational MCS and how the results validate the Research Framework and propositions, and by providing an overall evaluation of the key results. Next, the contributions of these results to the academic literature and their implications for practice are outlined. This is followed by the limitations of this study and the remaining issues to be solved in future research. Lastly, the conclusions for this study are presented. 


\subsection{Research Question - Implications for Organisational MCS}

The key results of this study can be evaluated in relation to the extent they have addressed the research question above. This study has identified a number of implications for organisational MCS in managing the volatilities and pressures associated with the GCCPs. First, generators need to make changes in their MCS to enable the planning and implementation of their organisation's environmental strategies. Multiple types of controls and control systems needed to be adopted. To implement emissions management, internal energy efficiency strategies and internal restructuring, generators needed to establish and utilise the internally-oriented strategy MCS component group that contains diagnostic controls and control systems, belief systems, boundary controls, and cross-functional systems. To realise the changing strategic direction in their political, generation, retail, and carbon credit strategies due to the GCCPs, generators employed externally-oriented strategy MCS components including interactive and enabling control systems and risk monitoring systems. Further, the use of legitimising MCS components was essential to enable generators to implement their chosen environmental policy, emissions disclosure, carbon neutrality and customer energy efficiency strategies, and respond effectively to GCCP-related institutional demands and pressures.

The second implication is that generators need to vary the degree of different MCS component group use, depending on the level of strategy proactiveness and strategic emphasis assigned to particular environmental strategies. In general, to implement more proactive environmental strategies in response to the multiple GCCP-related volatilities and pressures, generators needed to establish a higher number of controls and control systems and use MCS information with a broader scope and at more strategic level. However, in response to regulatory uncertainty and potential ETS changes, generators may reduce the level of proactiveness relating to political and generation strategies, and thus make less use of enabling and interactive control systems. Regarding the degree of strategic emphasis, if a renewable generator prioritises a renewable investment strategy, it requires more interactive use of its external fuels economics monitoring system and an enabling HRM system to develop internal renewable expertise. Similarly, as a thermal generator places more strategic emphasis on emissions management and carbon credit strategies to prepare for the upcoming ETS, such MCS components as the generation emissions monitoring systems and carbon-related budgetary controls will receive more use at top management levels. 
Thirdly, as with the strategy objectives, organisational MCS can be used for multiple objectives including competitiveness, legitimacy, and cost control. However, not all controls and control systems served these objectives simultaneously. Some MCS component types are geared towards competitiveness (externally-oriented strategy MCS), while some component types focus on cost control (internally-oriented strategy MCS), and the remaining component types serve to legitimise the generators to external stakeholders (legitimising MCS). Consequently, in designing their MCS, organisations need to be aware of the possible multiple objectives that MCS serves. Furthermore, to ensure that organisations can respond effectively to the diverse volatilities and pressures associated with the GCCPs, they need to establish a wide range of MCS component types and groups as identified in Chapter 8. It is also important that organisations clearly identify what environmental strategy (or mixture of strategies) and strategy objectives each of these MCS component types and groups support. This ensures that their environmental strategies are properly implemented and their objectives achieved, thus enabling organisational performance to be maintained, or enhanced in a GCCPfocused context.

Fourthly, while generators pursue common MCS objectives, the importance of these objectives need to be customised to match the primary objectives motivating each generator's environmental strategic changes. Accordingly, due to their generation emission profiles and the resulted strategic focus on carbon cost control, the primary MCS objective within the thermal generators is to reduce carbon costs. In contrast, consistent with their renewable assets and expertise and competitiveness-focused strategic changes, renewable generators need to employ their MCS primarily to support the development and maintenance of their green competitiveness. Other objectives, such as legitimacy, operating costs, and transactional costs, may be assigned secondary importance within the generators' organisational MCS. Therefore, the individual generators need to balance the competitiveness, cost control, and legitimacy objectives within their own organisational MCS in order to ensure that their MCS design and use fit their strategy objectives and strategic emphasis.

In summary, this study's results have identified four implications relating to how organisational MCS can be customised in order to achieve the strategic choices organisations make to manage the volatilities and pressures arising from GCCP-related environments. Accordingly, it is suggested that organisations need to design a wide range of controls and control systems and use them appropriately in accordance with the 
level of strategy proactiveness and strategic emphasis they adopt. Simultaneously, organisations should focus MCS design and use on the primary objectives that are the highest concern to the organisation from an environmental strategic perspective. These implications are likely to be useful for practitioners and policy-makers in helping organisations adapt to a carbon-constrained world (as discussed in Section 9.5.2). Furthermore, to understand this study's implications from a theoretical perspective, the next section discusses the results in relation to the Research Framework and

propositions presented in Chapter 4. Doing so also provides a basis for further evaluation of this study's results.

\subsection{Validation of the Research Framework and propositions}

The Research Framework (Figure 4-2) suggests that the GCCPs planned or introduced from 2002 to 2009 influenced generators' external environments, which in turn would lead to a change in their environmental strategies and organisational MCS. Furthermore, GCCP-related internal characteristics and capabilities are argued to impact on the generators' choice of environmental strategies. This framework results in four propositions.

P1: External GCCP-related economic and institutional drivers impact on generators' competitiveness, costs and legitimacy;

P2: Internal GCCP-related characteristics and capabilities impact on generators' competitiveness, costs and legitimacy;

P3: The impacts of GCCP-related external and internal drivers in turn lead generators to change their environmental strategies;

P4: The change in environmental strategies requires corresponding modifications in organisational MCS.

The results gained from the analysis of the multiple-case study data are summarised in Figure 9-1. Overall, these results support the four propositions and the key drivers included in the Research Framework. Moreover, a number of additional drivers are found and new relationships between GCCP-related external and internal drivers, environmental strategies and MCS identified. The new drivers are indicated by italics in Figure 9-1 and new relationships portrayed by dotted lines. 
Figure 9-1: Results framework

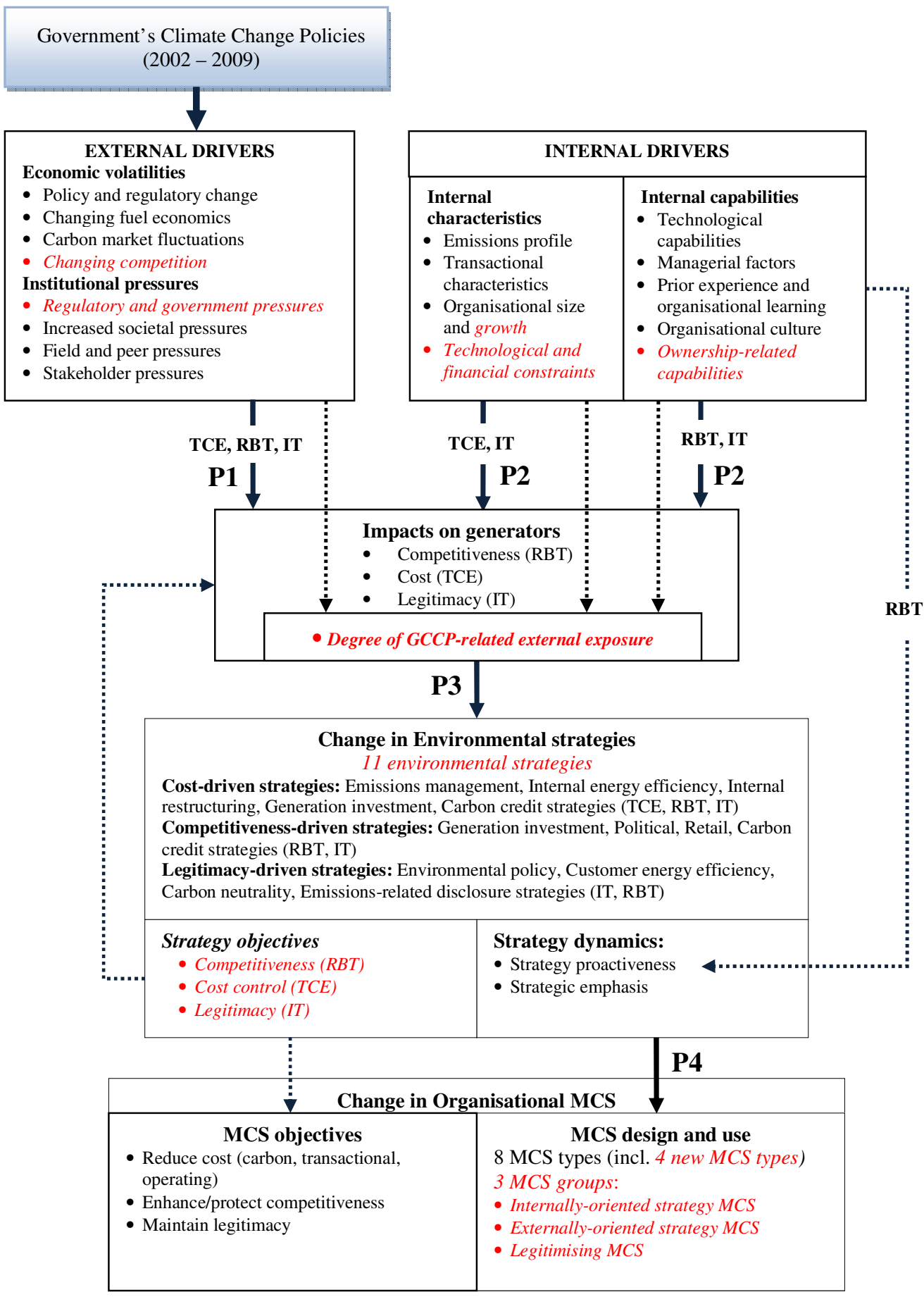

Note: Arrows do not indicate causal relationships

\section{Proposition 1}

In relation to P1, the results suggest that the changes in GCCPs from 2000 to 2009 led to changes in the generators' external environments. These changes resulted from increasing volatilities in the generators' economic environments due to the GCCPs and the associated policy and regulatory change, changing fuel economics, carbon market 
fluctuations and changing competition. External economic volatilities primarily affected generators' competitiveness and costs (RBT and TCE). The generators were also exposed to GCCP-related institutional pressures, namely, regulatory and government pressures, societal pressures, stakeholder pressures, and field and peer pressures. These institutional pressures in turn had implications for generator's social legitimacy (IT). Thus, P1 is supported in that external drivers are found to impact on generators' competitiveness, costs and legitimacy. Furthermore, new external drivers of changing competition (an economic volatility), and regulatory and government pressures (institutional pressures), were identified. Additionally, results indicate that GCCPrelated external drivers potentially drive the degree of the generators' external exposure to the GCCPs. As these volatilities and pressures intensified over time, from 2000 to 2009, due to GCCP changes, the degree of GCCP-related external exposure perceived by all five generators increased.

\section{Proposition 2}

Consistent with P2, the results suggest that internal characteristics and capabilities impacted on the generators' competitiveness, costs, and social legitimacy. Different from the prior literature and the proposed framework, the results clearly distinguish between two types of GCCP-related internal drivers: internal characteristics and internal capabilities. Accordingly, internal characteristics, namely, emissions profile, transactional characteristics, organisational size and growth, and technological and financial constraints increased the carbon, transactional and operating costs (TCE) for generators and threatened their social legitimacy (IT). In contrast, internal capabilities including technological capabilities, managerial factors, prior experience and organisational learning, organisational culture, and ownership-related capabilities were found to enhance the generators' market competitiveness (RBT) or social legitimacy (IT). The results also suggest a new internal characteristic, namely, organisational growth, and a new internal capability, ownership-related capabilities, which have not been examined in previous studies and was not part of the research framework.

Relationships were also found between GCCP-related internal drivers and external drivers; the internal drivers moderated the degree of generators' exposure to the external drivers. Internal characteristics increased the negative impacts of the external drivers on the generators by, for example, disadvantaging them in regards to changing fuel economics and carbon market fluctuations and exposing them to stronger governmental and societal pressures. In contrast, internal capabilities increased the positive impacts of 
the external drivers on some generators by creating a market advantage for them, and/or reducing their exposure to carbon-focused institutional pressures. These results relating to the moderating role of internal factors on external exposure are insights not available from prior literature.

\section{Proposition 3}

The impacts exerted by GCCP-related external and internal drivers on organisational costs, competitiveness and legitimacy are found to lead to changes in environmental strategies undertaken by the generators. Eleven different environmental strategies were found to be used by the generators in response to GCCP-related external and internal drivers. These strategies were:

(1) Environmental/ sustainability policy;

(2) Generation investment strategy;

(3) Retail strategy;

(4) Emissions management strategy;

(5) Internal energy efficiency strategy;

(6) Emissions-related disclosure strategy;

(7) Customer energy efficiency strategy;

(8) Pursuit of carbon neutrality;

(9) Carbon credit strategy;

(10) Political strategy; and

(11) Internal restructuring.

Pursuing these different strategies enabled the generators to respond to the multiple volatilities and pressures arising from the GCCPs and manage the influence of internal characteristics and capabilities on their respective organisations.

Furthermore, the eleven environmental strategies adopted by the generators to respond to the GCCPs and their associated volatilities and pressures can be classified into three groups: cost-driven, competitiveness-driven, and legitimacy-driven (Figure 9-1). Costdriven strategies were adopted with a primary objective to reduce carbon, operating and transactional costs arising from the GCCPs. They are driven strongly by internal characteristics and institutional pressures and to a lesser degree by external economic volatilities. This suggests that environmental strategies for cost control need to be formulated within the wider institutional norms and expectations (a combined TCE and IT view). Competitiveness-driven strategies were motivated by a need to maintain and enhance organisational competitiveness in a GCCP context and primarily driven by 
external economic volatilities and internal capabilities, and to a lesser degree by institutional pressures. This indicates that generators sought to preserve their competitiveness within the bounds of their institutional environments and internal resource availability, providing support for an integrated RBT and IT perspective. Legitimacy-driven strategies were adopted in response to the increasing GCCP-related institutional pressures and simultaneously enabled by a number of internal capabilities, thus being consistent with a combined IT and RBT view. While previous studies tend to focus on one type of environmental strategy (emissions management, retail strategy, or political strategy), the study found many types of GCCP-related environmental strategies that were simultaneously adopted by generators. This study's attempt to classify different environmental strategies into three categories is also a first in the literature.

Consequently, the choice and implementation of environmental strategies within the five generators were motivated by three objectives: competitiveness, cost control and legitimacy, confirming the insights from RBT, TCE and IT respectively. However, the three objectives were not of equal importance to the generators. Accordingly, the changes in environmental strategies made by the thermal generators focused on carbon cost control, while renewable generators modified their environmental strategies primarily to maximise competitiveness. In contrast, maintaining legitimacy in a GCCP context was of secondary importance to all generators. The results regarding strategy objectives provide a further explanation of P1, P2 and P3 as they suggest that generators undertook environmental strategies with these specific objectives in mind, to mitigate the impacts of external and internal divers on their organisation.

This study also found support for the two aspects (or dynamics) of environmental strategy change as suggested in the research Framework; level of strategy proactiveness and change in strategic emphasis. Over time (from 2000 to 2008), the generators moved to more proactive (early and wide-scope) environmental strategies, corresponding to the increased managerial awareness of organisational GCCP-related external exposure. However, from October 2008 onwards, some generators reduced their level of strategy proactiveness in response to the regulatory uncertainty associated with the new government's ETS review. Variations in strategy proactiveness across the generators appear to be associated with differences in the degree of perceived external exposure to the GCCPs and the degree of total internal capabilities. The strategic emphasis or attention top management placed on environmental strategies also shifted over time, 
consistent with changing external volatilities and pressures resulting from the various GCCP changes from 2000 to 2009. There were variations in the strategic emphasis across the generators within each time period, which potentially reflect inter-generator differences in the impact of internal characteristics and capabilities. Additionally, these results indicate that internal capabilities had an influence on the level of proactiveness of environmental strategies. Some internal capabilities, including managerial factors, organisational culture, and ownership-related capabilities, enable the generators to execute more proactive environmental strategies in response to the GCCPs (a RBT view). Hence, these results provide support for a RBT-based explanation in the examination of the relationship between internal capabilities and environmental strategies. Further, this direct relationship is a new insight that was not proposed in the Research Framework.

\section{Proposition 4}

Changes in environmental strategies in response to GCCP-related external exposure were found to drive organisational MCS design, use and objectives. MCS design and use are captured in the MCS component types and groups. Consistent with Simons (1991), empirical evidence was found for four MCS component types which were critical in the implementation of environmental strategies. These were boundary controls, belief systems, diagnostic controls and control systems, and interactive controls and control systems. Additionally, four new MCS component types were identified and named as: enabling controls and control systems, cross-functional controls and control systems, legitimising controls and control systems, and risk monitoring systems. The degree of use of each of these eight MCS component types changed over time and varied across the generators in conjunction with the level of strategy proactiveness and strategic emphasis undertaken. While previous studies recognise that organisations may use multiple controls and control systems to support their strategies, this study's clear identification of eight different MCS component types presents a theoretical contribution to the literature.

The eight MCS component types can be classified into three main MCS component groups underpinned by different MCS objectives. The first group is internally-oriented strategy MCS which contains belief system, boundary controls, diagnostic controls and controls systems, and cross-functional controls and control systems. This group aimed at controlling carbon, operational and transactional costs by facilitating and monitoring the implementation of emissions management, internal energy efficiency and internal 
restructuring strategies, and generation investment and carbon credit strategies (in thermal-based generators). Thus, support for a TCE explanation is provided. The second group of interactive and enabling control and control systems and risk monitoring systems is classified as the externally-oriented strategy MCS. This component group facilitates the planning and implementation of competitiveness-driven strategies including generation investment, retail, political and carbon credit strategies. Thus, consistent with a RBT perspective, the design and use of this second MCS group is aimed at maintaining and enhancing generators' competitiveness. In contrast, the third component group, legitimising MCS legitimate the generators in the face of GCCPrelated governmental and societal pressures, thus providing support for an IT-based explanation. The use of this MCS component group ensures the achievement of legitimacy-driven strategies, which include carbon neutrality, environmental policy, customer energy efficiency and emissions-related disclosure strategies. Furthermore, as with environmental strategies, the classification of controls and control systems into the three MCS component groups is new and has not been employed in the prior literature. These findings also provide empirical support for prior research (e.g. Langfield-Smith, 1997, Chenhall, 2003) regarding the importance of strategy driving MCS design and use.

Consistent with the prior literature, the results indicate there are multiple objectives underlying MCS design and use. Internally-oriented strategy MCS component group, externally-oriented strategy MCS component group, and legitimising MCS component group were used respectively to reduce carbon, transactional and operating costs, to gain competitiveness, and to maintain legitimacy. However, in contrast to previous studies, this study did not find that organisational MCS component groups were used specifically to manage external risks and uncertainty, or to gain political power. The objective of the risk monitoring systems is to provide information on external environmental changes to enable generators to revise and plan their environmental strategies to maintain competitiveness. Similarly, the interactive use of accounting in political strategy was not to gain political power, but to protect or enhance competitiveness. Furthermore, substantial differences between the five generators in relation to the primary objectives underlying their MCS were identified, with the objectives of each being in sync with those motivating the changes in its environmental strategies. While the direct relationship observed between strategy objectives and MCS objectives was not part of the Research Framework, it is consistent with the P4 
argument and prior research that the choice of environmental strategies drives organisational MCS.

\subsection{Key results: Overall evaluation}

The results discussed above address the research question by providing a number of implications for organisational MCS arising from the strategic choices generators make to manage the volatilities and pressures associated with GCCP changes. Evidence is provided that confirms GCCP-related volatilities and pressures interact with internal characteristics and capabilities to drive the generators' external exposure to the GCCPs, by increasing compliance, operating and transactional costs, enhancing market competitiveness, and ensuring societal legitimacy. To respond to this external exposure, the generators formulate multiple environmental strategies and used them with varying degrees of proactiveness and top management emphasis. In turn, these strategic changes have implications for the design, use and objectives of organisational MCS. New types of controls and control systems are established to assist in the planning and implementation of specific environmental strategies. The degree of use of these controls and control systems and their objectives also changed to reflect the strategic emphasis and the level of proactiveness within each generator in each time period. Overall, these results support the proposed Research Framework and the four propositions. They also confirm the relevance of using RBT, TCE and IT simultaneously in the examination of organisations' environmental strategies and MCS changes in a GCCP-focused context. Additionally, a number of new insights were gained:

1. Other drivers of environmental strategies identified are: changing competition (external economic volatility); regulatory and government pressures (external institutional pressures); organisational growth (internal characteristic); technological and financial constraints (internal characteristics); and ownershiprelated capabilities (internal capabilities).

2. GCCP changes led to an increasing degree of generators' organisational exposure to external volatilities and pressures, reflecting an increasingly complex and volatile operating environment in which the generators operate, due to the introduction of the GCCPs.

3. Internal characteristics and capabilities moderate the degree of a generator's exposure to GCCP-related external volatilities and pressures. 
4. Generators adopted multiple environmental strategies to manage the impacts exerted by GCCP-related external and internal drivers and these strategies can be classified into three groups: competitiveness-driven; cost control-driven; and legitimacy-driven.

5. These multiple strategies and strategy groups were underpinned by three key objectives; controlling carbon and transactional costs, enhancing competitiveness, and maintaining legitimacy.

6. A generator's strategy proactiveness and strategic emphasis also changed over time in accordance with the changing external exposure to the GCCPs, to ensure that it could effectively respond to and manage the impacts of GCCP-related external and internal drivers.

7. A wider range of MCS component types (8) were used to support environmental strategies than the four previously identified by Simons (1991) and the associated literature. These different MCS component types can be broadly classified into three MCS component groups, based on their relationships with environmental strategies: externally-oriented strategy MCS; internally-oriented strategy MCS; and legitimising MCS.

8. The degree of each MCS component type and group's use also depends on the level of strategy proactiveness and strategic emphasis a generator places on particular environmental strategies within each time period.

9. While organisational MCS as a whole may serve multiple objectives, each MCS component type and group serve a small number of objectives, that are consistent with the primary objectives of changes in environmental strategies.

These additional insights highlight the differences from prior research and suggest a number of contributions that this study can make to the literature. These contributions are discussed in the next section.

\subsection{Contributions}

This study's results contribute to the academic literature and have implications for practice. Theoretical contributions to the academic literature are discussed next, followed by the practical implications. 


\subsubsection{Theoretical contributions}

It is stated at the end of Chapter 3 that a number of gaps exist in the academic literature. This study's results provided empirical evidence that contributes to the narrowing of these gaps.

Gap (1): Lack of understanding about organisations' environmental strategies in a GCCP-focused context

In relation to Gap 1, one of the key insights gained from this study is that generators not only modify existing environmental strategies, but also establish new ones to respond to the multiple volatilities and pressures associated with the changing GCCPs. Additionally, they changed the level of strategy proactiveness, and shifted the strategic emphasis among environmental strategies. Importantly, these multiple strategies were motivated by differing objectives (cost control, legitimacy, or competitiveness) to ensure generators manage their organisational exposure to the GCCPs effectively.

Gap (2): Lack of a theoretical underpinning to explain the mechanisms by which economic factors influence strategy and MCS choice

To address Gap (2), this study assessed the economics-based theoretical underpinnings of environmental strategies and MCS. Results suggest that GCCP-related external and internal economic drivers (in particular, regulatory and policy change, carbon market fluctuations, emissions profile, and transactional characteristics) can affect generators' carbon-related, operating and transactional costs, thus providing support for a TCE perspective. In response, emissions management, internal energy efficiency, internal restructuring, generation investment and carbon credit strategies were adopted to focus on cost control. To implement these strategies, an array of internally-oriented strategy MCS components were designed and used. Therefore, by using TCE, this study highlights how external and internal economic drivers influence environmental strategies and identifies which types of organisational MCS components were used to ensure cost control and internal efficiency in responding to GCCP-related external volatilities and pressures.

Gap (3): Little insight into how a longitudinal period of GCCP change affects organisations' external and internal environments, and the potential inter-relationships between external and internal drivers in driving environmental strategies and MCS responses 
This study found that a prolonged period of GCCP change brought about changes in the economic and institutional environments in which generators operated. The GCCPs led to many additional volatilities and pressures such as carbon market fluctuations, changing fuel economics, and increasing governmental and societal pressures associated with emissions reduction and disclosure. Consequently, generators were exposed to increasing degrees of exposure to the GCCP-related external volatilities and pressures over time. To manage this exposure, generators adopted multiple environmental strategies such as generation investment, carbon credit, retail, political and internal energy efficiency strategies. They also changed the level of strategy proactiveness and top management placed different levels of emphasis on particular strategies in order to effectively respond to the volatilities and pressures dominant within each period of GCCP change.

This study also offers insights regarding the relationships between external and internal drivers in driving environmental strategies. Internal capabilities and characteristics are found to moderate the degree of external exposure which in turn drove generators' environmental strategic responses. Internal characteristics led to more negative impacts being exerted by the GCCP-related external drivers on the generators, while internal capabilities enhanced the positive impacts. Furthermore, the generators with strong internal capabilities such as renewable inheritance assets and expertise, top management commitment and supportive organisational culture were able to pursue more proactive environmental strategies.

Gap (4): Limited understanding regarding the link between environmental strategy and organisational MCS, and the role of MCS in strategy planning

Further empirical evidence on the link between strategy and MCS is provided. Consistent with prior studies, some of the controls and control systems found were driven by a need to control specific environmental strategies and ensure cost reduction, i.e. internally-oriented strategy MCS component group. However, other control and control systems were used to build internal capabilities for a competitive advantage or to provide information to facilitate strategic planning. These are the externally-oriented strategy MCS component group that includes enabling and interactive controls and control systems and risk monitoring systems. Furthermore, some controls and control systems (legitimising MCS component group) were specifically used by the generators to legitimate themselves to external stakeholders. Therefore, in planning and controlling the generators' environmental strategies, organisational MCS design and use were not 
only driven by the need for internal efficiency and cost control as traditionally recognised in the literature, but simultaneously motivated by a wider range of objectives including increasing competitiveness and gaining legitimacy. Additionally, there is a close coupling between strategy and MCS in that the predominant objectives underlying strategy choice determine the predominant objectives of MCS design and use within each generator.

In addition to narrowing the above gaps in the literature, this study makes a theoretical contribution by developing a multi-theory framework to explain generators' organisational adaptation to external environmental regulatory changes. This framework argues for the need to consider multiple theories in this study, each of which offers a distinctive and valuable insight that contributes to a comprehensive and holistic understanding of environmental strategy and MCS changes in a GCCP context. The simultaneous use of TCE, IT and RBT explains the different mechanisms by which the GCCPs influence environmental strategies, as well as the complexity identified in environment-related MCS design, use, and objectives.

A multiple-theory framework also allows for theory triangulation in determining the relative economic and social impacts of GCCP-related external and internal drivers, and the relative importance of different strategy and MCS objectives in responding to these drivers. Therefore, the impacts of external drivers on environmental strategies can be explained from a TCE, RBT or IT perspective, while internal capabilities affect environmental strategies from a RBT or IT view and internal characteristics from a TCE or IT view (Figure 9-1). Theory triangulation further enables an understanding of why some organisations (e.g. renewable generators) primarily focus on managing GCCPrelated competitiveness (RBT), while others (e.g. thermal generators) consider carbon cost control of paramount importance (TCE) in their strategy formulation and MCS design and use.

A further contribution is this study's classification of controls and control systems into 8 MCS component types and 3 MCS component groups. This classification captures a wider range of controls and control systems than previous studies and provides a useful mechanism to assess the role played by organisational MCS in:

i) the planning and implementation of environmental strategies with different focuses, and

ii) monitoring and responding to external volatilities and pressures. 
Consequently, this classification facilitates an assessment of the match between organisational MCS and GCCP-related environmental strategies and external volatilities and pressures and enables discrepancies or mismatches to be identified and potentially explained. This, along with the other results, has practical implications for organisations, policy-makers and practitioners, as discussed next.

\subsubsection{Contributions to practice}

In Chapter 8 (8.4) and Section 9.2.1, the implications for organisational MCS arising from strategic choices generators make in response to the changing GCCPs have been discussed in relation to the research question. In this section, the implications of this study for practice are discussed in further detail, by relating back to the practical motivations highlighted in Chapters 1 and 2. Each motivation gives rise to one or a number of implications, as presented next.

Motivation (1): Potential identification of the GCCP-related factors in the generators' external and internal operating environments that drive generators' decisions to change their environmental strategies.

The generators undertook a diagnosis of the potential volatilities and pressures associated with the planned GCCPs, as well as assessing their internal characteristics and capabilities. As a result, the generators recognised that the GCCPs could exert multiple external economic volatilities and institutional pressures, which would not only affect their carbon and operating costs but also their market competitiveness and social legitimacy. Generators were also aware that their individual internal characteristics and capabilities moderated their exposure to the GCCPs. To prepare for a carbonconstrained operating environment, the generators considered their GCCP exposure in their strategy planning processes and formulated appropriate climate change plans and environmental strategies. In doing so, they modified existing strategies (e.g. their generation and retail strategies) and established new ones (e.g. internal energy efficiency, emissions management and carbon credit strategies). Furthermore, their strategic choices and responses were designed to take advantage of existing internal capabilities, as well as reduce the impacts of internal characteristics (e.g. emissions profile). This implies that environmental strategies need to match firm-level internal characteristics and capabilities and thus there is no one-size-fits-all solution, despite the fact that organisations may operate in similar external economic and institutional environments. 
Motivation (2): A potential understanding of how the generators have responded, and adapted organisationally to the changing external environments associated with the planned GCCPs.

Most generators have increased their renewable investments and undertaken internal energy efficiency initiatives in response to the planned GCCPs. Most of them have also moved towards greening their retail brand and reputation, adopted carbon neutrality programmes, and encouraged customer energy efficiency. To protect their competitiveness, all generators participated actively in the GCCP policy processes. However, not all generators changed in the same manner. The generators with a high degree of exposure to the GCCPs, in terms of both positive and negative impacts, adopted more proactive, wider-scoped environmental strategies which involved multiple organisational functions in the strategy planning and implementation processes. In contrast, generators experiencing lower exposure tended to be slower in making change or their changes were not as extensive or as wide in scope. Over time, as the generators recognised the increasing volatilities and pressures associated with the GCCPs, the degree of strategy proactiveness and resulting resource assignment grew. Furthermore, top management emphasised a number of selected environmental strategies that could help them respond effectively to the predominant volatilities and pressures within each phase of GCCP change. Hence, a key implication is that organisations make varying strategic and operational changes, that are dependent upon their firm-specific external exposure to the GCCPs and how such exposure changes over time.

Additionally, the results indicate that the GCCPs from 2000 to 2009 have been relatively successful in triggering strategic and operational changes within the generators. All the generators studied, including the thermal ones, have moved towards a renewable-focused generation investment strategy, in response to the carbon pricing signals provided by the GCCPs. This suggests that the generators are increasingly adopting lower-emitting generation technologies, and hence, contributing to the reduction of the sector and the nation's total emission levels. There is also evidence that generators also promoted energy efficiency behaviour and practices among its employees, suppliers, and customers. However, the ETS review and modifications since October 2008 have caused a delay in the generators' renewable investment decisions. Therefore, the tentative implication for policy-making is that a strong carbon pricing signal and a positive regulatory environment for renewable investments are possibly 
required in order to encourage substantial renewable development and thus significant emission reduction by the generators.

Motivation (3): An assessment of the implications for organisational MCS to enable and support the strategic choices generators make in relation to the GCCPs.

Generators changed their MCS to support the implementation of their environmental strategies. Some of the controls and control systems found in the five generators were designed and used to meet short-term compliance obligations, such as emissions monitoring and reporting, and carbon credit purchases. However, generators increasingly used their organisational MCS to assist the planning and implementation of environmental strategies (e.g. emissions management, generation investment, internal energy efficiency and carbon neutrality strategies). These strategies, and associated MCS components, led generators to reduce their emissions levels and thus contributed to the achievement of long-term cost control and competitiveness. Additionally, some controls and control systems were used to directly respond to institutional expectations and pressures. Thus, these results imply that rather than merely focusing on compliance activities, generators need to employ a wider range of controls and control systems and use them at a more strategic level to enable internal change and the management of organisational performance in the face of the multiple volatilities and pressures associated with the GCCPs.

Motivation (4): A practical insight regarding why generators chose and undertook the strategic and MCS changes they did (in response to the GCCPS).

The three objectives of competitiveness, cost control and legitimacy, were found to motivate environmental strategy choices and MCS design and use. These multiple objectives ensure that generators can effectively respond to and manage the impacts exerted by multiple GCCP-related external volatilities and pressures on their organisation. However, while a generator may seek to achieve all three objectives in their environmental strategy and MCS changes, the relative importance of these objectives varies across each generator. Consequently, in a GCCP-focused context, thermal generators with high emissions levels, tend to use their strategies and MCS primarily to control carbon costs. In contrast, strategic and MCS responses undertaken by renewable generators are primarily motivated by the potential to enhance green competitiveness due to their internal renewable-based capabilities. Hence, the different levels of importance assigned to the different objectives enable management to focus on 
managing the key impacts exerted by GCCP-related internal characteristics and capabilities.

The above insights are useful for professionals and practitioners working in the areas related to GCCPs and climate change as well as the Government and regulators. For managers, accountants and external consultants, they need to understand the possible impacts of the GCCPs on organisational operations and take them into consideration in planning strategy and MCS changes. Furthermore, these results potentially give managers some guidance of how to customise their MCS so as to support strategy achievement and respond effectively to the volatilities and pressures emerging from the GCCPs. In doing so, it should highlight to managers the importance of aligning MCS design, use and objectives with strategy choice and objectives. The results also allow an assessment of whether and to what extent the changes in GCCPs over the years and the prospect of an ETS have triggered renewable investments and energy efficiency from the electricity generators. Based on this assessment, the Government and policy makers can evaluate the effectiveness of GCCPs, as economic instruments, in bringing about behavioral changes and achieving the desired environmental and economic objectives, for example, reduced emissions levels and lower Kyoto liabilities. They can also assess the change in level of generators' engagement after the moderation of the Original ETS (since October 2008). In doing so, appropriate policy advice and adjustments can be made to improve the potential outcomes of the GCCPs.

\subsection{Limitations and future research}

Despite its contributions, this study has a number of limitations. These limitations correspond to the opportunities for future research. Firstly, this study focused on top managers and did not examine the perceptions and the role played by other staff. This is partly due to the nature of the research question and corresponding methodological choice to collect data relating to the strategic planning and decision-making at a top management level. Furthermore, the reliance on interview and documentary data meant that the researcher was not able to observe the actual implementation of environmental strategies and actual use of organisational MCS, especially at operational levels. Previous studies suggest that actual MCS design and use can differ from the intended or original model due to internal and external barriers to the MCS change process (Kasurinen, 2002). Additionally, individual business units may use different controls and control systems from those used at top management levels due to potential 
disagreement between the top and lower management levels regarding the appropriateness of specific MCSs (Govindarajan, 1988; Malina and Selto, 2001). Future research can examine the actual implementation process of environmental strategies and MCS changes in relation to GCCPs, as well as investigate the role of lower level managers and other staff in this process.

Secondly, this study did not investigate the actual impacts of strategy and MCS changes on organisational performance. It only examined the understanding of top management regarding the potential impacts of GCCP-related external and internal drivers on the generators and how their environmental strategies and MCS were modified in accordance with this understanding. The results provide some evidence about how strategy and MCS changes in previous periods influenced organisational performance in later periods. For example, renewable investments made in the 2002-2005 period earned carbon credits and the renewable expertise gained allowed some generators to develop a competitive advantage in carbon trading and renewable energy in later periods. However, there is little evidence of how organisational revenue and profitability were enhanced or decreased due to the strategy and MCS changes made to respond to the GCCPs. A type of time-series data and potentially a higher number of cases/organisations may be required to enable the analysis of the association between strategy and MCS changes and organisational financial performance. In doing so, an understanding of the impacts of GCCP-related strategy and MCS changes on organisational performance could be gained.

Thirdly, individual theories used in this study were not utilised to their fullest potential. This study focused on using a combination of TCE, RBT and IT to maximise their complementarities and on providing diverse and rich insights into GCCP-related strategic choices and their implications for organisational MCS. However, due to this focus, the study was not able to determine what the TCE-based implications of the GCCPs were for institutional pressures, e.g. the extent that GCCP-related economic impacts affected, or shifted, societal and stakeholder pressures. It was also not the purpose of this study to use TCE to assess the impact of individual generator's strategic choices on their organisational transactional costs, nor to compare the relative transactional costs incurred by different generators when confronted by the same source of GCCP-related external volatility/pressure. These potential uses of TCE could be employed in future research related to GCCPs, or similar economic interventions. Similarly, within its scope, this study did not use IT to identify the potential different 
strategic responses that generators can adopt to respond to the same source of institutional pressures (Oliver, 1991). While the way that generators responded to GCCP-related pressures was captured to some extent by examining the degree of strategy proactiveness and top management emphasis on selected environmental strategies, it does not represent the specific strategic response categories suggested by Oliver (1991). Future research could investigate these responses and explain why organisations undertake varying responses despite being exposed to a similar source of institutional pressures. Furthermore, when using RBT, this study, to a large extent, treated the generators' internal capabilities as being independent from one another and did not distinguish between static and dynamic resources or capabilities (Fiol, 2001; Kraaijenbrink et al., 2010). Future research could employ RBT to provide more indepth insights into the role played by different categories of internal resources and capabilities and the interaction among them during the implementation of environmental strategies and the maintenance of an organisation's competitive advantage.

Fourthly, one can argue that this study's generalisability is limited because of its case focus (five generators) and single industry examination. However, this study enhanced its internal validity by choosing cases through the application of replication logic, using reflective strategies across the enquiry process, and triangulating results between different data sources and theories. Since the cases examined covered the key players in wholesale and retail electricity markets (accounting for more than $90 \%$ of the market share), this study's results could be generalised to the electricity generating sectors in other countries, which are also planning or enforcing some form of GCCPs. Furthermore, many of the volatilities and pressures imposed by the GCCPs on generators, such as policy and regulatory change, and governmental and societal pressures associated with emissions reduction, can be argued to be relevant to other New Zealand organisations. Consequently, this study's Research Framework can be applied to other industries and sectors which have compliance obligations under the GCCPs or are significantly influenced by them (e.g. due to an intensive use of electricity or fossil fuels). The employment of this framework in future research to analyse and interpret the data from other industries and sectors would also enhance the external validity of this study's results.

Fifthly, this study has only examined changes in generators' environmental strategies and organisational MCS during the period from 2000 to 2009, which was before the actual enforcement of the ETS (July 2010). Thus the results are limited to insights into 
the internal changes within the generators to prepare for an ETS. Future research could investigate whether these strategic and MCS adaptations are maintained or modified once the ETS comes into operation.

Lastly, this study has examined the relationships between external and internal drivers, environmental strategies and organisational MCS in an assumed top-down direction. However, it is probable that these relationships are bi-directional or circular. There is some evidence in this study (7.5.1) that the choice of particular environmental strategies led to the development of internal capabilities. Further, the environmental strategies adopted by generators potentially leads to changes in their external environments. For example, an emissions-related disclosure pursued by a generator can "raise the bar" in terms of societal expectation of corporate disclosure practices. Similarly, a green branding campaign can intensify retail market competition. It is also possible that the adoption of particular controls and control systems, e.g. a carbon neutrality system, by one generator may be considered an industry best-practice and thus creates peer pressures for other generators to follow. These potential impacts of environmental strategies and organisational MCS on organisations' GCCP-related external and internal environments provide a fruitful avenue for future research.

\subsection{Conclusion}

The aim of this study was to examine the impacts of GCCP changes (from 2000 to 2009) on the environmental strategies and organisational MCS of New Zealand electricity generators. To achieve this aim, the research question investigated was: What are the MCS implications arising from strategic choices New Zealand electricity generators make in managing economic volatility and societal pressures resulting from a prolonged period of GCCP changes?

This study has addressed the research question by providing empirical evidence regarding the changes in generators' environmental strategies to respond to the volatilities and pressures associated with the GCCP changes and highlighting the implications of such strategic changes for organisational MCS design, use and objectives.

The key academic contribution of this study is in developing a multiple-theory framework that integrates TCE, IT and RBT to explain how and why GCCP drives an organisation's environmental strategies and associated MCS change. In doing so, this 
study demonstrates the relevance and complementarity of the three theories to provide rich insights into the dynamics and objectives underlying organisational adaptations to external environmental changes. This framework allows an understanding of why different external economic and societal factors motivate organisations to undertake different environmental strategies with varying objectives. It further provides insights into what types of controls and control systems support these different strategies and for what objectives. This framework can be employed in future research to assess the impacts of the GCCPs in other industries and countries.

The results have significant implications for practice. As the volatilities and pressures associated with the GCCPs are likely to increase, especially as the ETS comes into operation, organisations need to be aware of the degree of their organisational exposure to the GCCP-focused operating environment. This awareness is critical because it enables organisations to formulate appropriate strategic responses. Therefore, they need to identify the external volatilities and pressures arising from the GCCPs, and understand that these volatilities and pressures not only impose direct financial impacts on organisations, but also could affect their social legitimacy and competitiveness. Managers also need to assess their organisation's internal characteristics and capabilities and determine how these internal factors drive and affect the organisation's external GCCP exposure.

In formulating their environmental strategies, it is important for organisations to recognise that there is no one-size-fits-all strategic solution regarding how to respond to the GCCPs. To ensure that all their applicable GCCP-related volatilities and pressures are adequately responded to, organisations may adopt multiple environmental strategies simultaneously. The level of strategy proactiveness and top management emphasis on different strategies will vary over time and across organisations, corresponding to the changing external volatilities and pressures and the impacts exerted by internal characteristics and capabilities. Furthermore, some organisations will need to formulate their environmental strategies to focus more on controlling and reducing their compliance carbon costs due to their high emissions profile. Other organisations, with a low emissions level or no emissions, will not consider this objective important. Instead, these organisations will need to channel their strategic attention and resource allocation into creating a green competitive advantage. Additionally, due to increasing societal pressures associated with climate change and the need for carbon emissions reduction, all organisations should allocate adequate resources to emissions-related disclosure and 
carbon-focused strategies, in order to demonstrate their environmental responsibility and thus, maintain their legitimacy.

The implication for any organisational changes made in response to GCCPs, is that the MCS needs to be substantially modified in order to support organisations' environmental strategies. It is not sufficient for organisations to just establish compliance-focused control systems to satisfy their GCCP-related obligation requirements. Organisations need to design and use a wide range of MCS component types to assist with the planning and implementation of multiple environmental strategies and to enable the effective achievement of GCCP-related strategic objectives. Furthermore, managers need to ensure that organisational MCS is designed and used to achieve the same objectives that underline the choice of their environmental strategies.

In conclusion, it is recognised that climate change policies, such as a carbon tax or an ETS, are becoming important to organisations in New Zealand and internationally. These policies bring about additional volatilities and pressures that have the potential to affect organisations significantly and beyond the boundaries of traditional environmental regulatory frameworks. Strategically assessing the GCCP-related impacts on the organisation, and formulating appropriate environmental strategies and changing organisational MCS in response is a necessity for organisations to remain profitable, competitive, and legitimate. This study's results suggest that organisations should modify their environmental strategies to effectively respond to the volatilities and pressures arising from the GCCPs and the associated impacts of internal characteristics and capabilities. To effectively plan and implement these strategic changes, a wide range of controls and control systems (i.e. MCS component types) need to be established and used. Furthermore, the degree of use of different MCS component types will vary in accordance with the level of strategy proactiveness and the emphasis placed on environmental strategies by top management. Although the MCS can be used to achieve multiple objectives of competitiveness, cost control, and legitimacy, the relative importance of these objectives should be customised to match the primary objectives that underpin each organisation's environmental strategies. This study's results provide important insights for regulators and policy-makers into the potential economic and social impacts that climate change policies could exert on organisations. These results are also useful for practitioners, managers, and academics in understanding how organisations may respond to those impacts to ensure organisational survival in a carbon-constrained operating environment. 


\section{Appendices}

\section{APPENDIX A: PROPOSAL FOR RESEARCH PARTICIPATION}

\section{Introduction}

I am a PhD student in the School of Accounting and Commercial Law, Victoria University of Wellington. My $\mathrm{PhD}$ thesis is in investigating the impacts of the Government Climate Change Policies (GCCPs) on electricity generators and their strategies and management control system (MCS). More specifically, I am examining whether the introduction of the GCCPs results in additional volatility and pressures for generators and the implications of such volatility and pressures for GCCP-related environmental strategies and organisational MCS in order to ensure organizational survival and growth. Ethics Approval has been granted for this study.

\section{Desired participants}

I would like to invite you to participate in this research. The research primarily involves interviews of senior executives of New Zealand electricity generators, industry regulators, professionals and experts in risk management, carbon trading, sustainability and climate change. The potential interviewees are directors, senior and middle managers from your organisation who are involved in the planning and implementation of the climate change action plan and initiatives in response to the GCCPs or who have expert knowledge and experience in the electricity industry and the GCCP policy processes.

\section{Interview schedule}

A list of semi-structured interview questions will be sent to the interviewees at least two days prior to the interview. It is envisaged that the interviews will last about one hour. The time and location of the interviews will be arranged to the interviewees' convenience. The interview will be taped and transcribed afterwards.

\section{Data analysis and reporting}

The transcribed interviews will be analysed and form the basis of the findings of my research. The interviewees will not be individually identifiable in the thesis. All data will be kept confidential and exclusive to me and my supervisors, Dr Carolyn Fowler and Dr Chris Hunt. The thesis will be deposited in the library of Victoria University of 
Wellington. It is intended that data and results from the interviews will be also used for publications in scholarly journals, presentations at academic conferences, and potential reports and discussion papers to inform relevant policy planning and regulatory processes. At the conclusion of the project, a summary of findings will be sent to all the interviewees. We hope you will find this research of value to you.

\section{Contact details}

If you wish to participate in this research or desire further information, please contact me by email at Binh.Bui@vuw.ac.nz or phone 044636679 , or my supervisor Dr Carolyn Fowler at Carolyn.Fowler@ vuw.ac.nz (04 463 6506), or mail us at:

School of Accounting and Commercial Law

Victoria University of Wellington

PO Box 600, Wellington 


\section{APPENDIX B: SAMPLE INTERVIEW QUESTIONS}

\section{Questions for electricity generators}

1. What are the volatilities and pressures that the different GCCPs might bring, or have brought, to your organisation?

2. Do you perceive that your organisation is exposed to a different set of GCCPrelated volatility and pressures from other generators? What internal characteristics or capabilities do you think that might have driven such differences?

3. What strategic responses have been taken by your organisation to respond to these volatilities and pressures and manage organisational performance? Can you give me an example of such response in the different areas of the business?

4. Do you think the focus/importance on each of the above areas has changed due to the various changes in the GCCPs from 2002 to 2009 ?

5. Why does your organisation choose to undertake these specific responses? What are the key internal (characteristics or capabilities) that have enabled, or driven, these strategic responses? What benefits or objectives do you perceive that such responses may bring to the organisation?

6. How do you implement these GCCP-related strategic responses? What are the key human and non-human resources that are allocated to climate change and GCCP areas? Have you experienced any problems in implementing the climate change plan across the organisation?

7. What types of management control system (MCS) have been planned, or established within your organisation, to prepare for the GCCPs and support the above strategic responses?

8. How are controls and control systems used? What types of decisions does MCS information assist? At which level of management is such MCS information used?

9. What controls or control systems receive the highest top management attention within each period of GCCP change? Why?

10. What objectives (or roles) do you perceive the above controls and control systems are fulfilling within your organisation? 


\section{Questions for external organisations and stakeholders}

1. What are the risks and opportunities that the ETS brings about to the NZ economy, and in particular, the electricity industry? Would the potential electricity price increases due to the ETS influence the competitiveness of NZ businesses?

2. What are the major strategic and operational risks of an ETS for electricity generators? How do these differ across different generators?

3. How has your organisation been involved in the development of the GCCPs and in preparing the electricity generators for the introduction of such policies?

4. What role do you think that external organisations play in influencing businesses' response (including changes to their strategies and operations) to climate change and an ETS? Which organisation has been the most influential?

5. Do you think that the proposed ETS will lead to major strategic changes in electricity generators, including generation investment and competitive strategies? If yes, why is that so?

6. From your experience in electricity industry and emissions trading, what changes have you seen as happening within electricity generators to respond to and prepare themselves for the GCCPs?

7. Have the generators' responsiveness and reaction towards the GCCPs changed over the years (2002-2009)? If yes, what are the drivers for such change?

8. In order to monitor and manage the impacts of the GCCPs, what changes are required in regards to management control systems, e.g. the emissions internal reporting systems, carbon market monitoring system, carbon accounting system, budgetary and strategic planning processes? To which extent do you perceive that electricity generators have undertaken these changes?

9. Do you perceive that emissions-related disclosure by electricity generators has increased from 2000 to 2009 ? If yes, what are the drivers and objectives for such change?

10. Do you think that the introduction of the GCCPs such as an ETS will lead to the development of more sustainable business? For example, do you think that an ETS will lead to the adoption of lower-emissions technologies and behaviour? 


\section{References}

Abouzeid, K. \& Weaver, C. (1978), "Social responsibility in the corporate goal hierarchy", Business Horizons, Vol. 21, No. 3, pp. 29-3.

Abernethy, M. A. \& Chua, W. F. (1996), "A Field Study of Control System "Redesign": The Impact of Institutional Processes on Strategic Choice", Contemporary Accounting Research, Vol. 13, No. 2, pp 569-606.

Abernethy, M. A. \& Brownell, P. (1999), "The role of budgets in organizations facing strategic change: An exploratory study", Accounting, Organizations and Society, Vol. 24, No. 3, pp. 189-204.

Aerts, W. \& Cormier, D. (2009), "Media legitimacy and corporate environmental communication", Accounting, Organizations and Society, Vol. 34, No. 1, pp. 1-27.

Ahrens, T. \& Dent, J. (1998), "Accounting and organizations: Realising the richness of field research", Journal of Management Accounting Research, Vol. 10, No. pp. 1-40.

Ahrens, T. \& Chapman, C. S. (2006), "Doing qualitative field research in management accounting: Positioning data to contribute to theory", Accounting, Organizations and Society, Vol. 31, No. 8, pp. 819-841.

Álvarez-Gil, M. J., Burgos-Jiménez, J. \& Céspedes-Lorente, J. J. (2001), "An analysis of environmental management, organizational context and performance of Spanish hotels", Omega, Vol. 29, No. 6, pp. 457-471.

Anthony, R. N. (1965), Planning and control systems: A framework for analysis, Harvard University, Graduate School of Business Administration, Boston.

Arksey, H. \& Knight, P. (2002), Interviewing for social scientists: An introductory resource with examples, Sage, Thousand Oaks, CA.

Armstrong, P. (1985), "Changing management control strategies: The role of competition between accountancy and other organisational professions", Accounting, Organizations and Society, Vol. 10, No. 2, pp. 129-148.

Arnold, D. O. (1970), "Dimensional sampling: An approach for studying a small number of cases", American Sociologist, Vol. 5, No. 2, pp. 147-150.

Azzone, G. \& Bertelè, U. (1994), "Exploiting green strategies for competitive advantage", Long Range Planning, Vol. 27, No. 6, pp. 69-81.

Bansal, P. (1995), "Why do firms go green? The case for organisational legitimacy", DPhil Thesis, University of Oxford.

Bansal, P. (2005), "Evolving sustainably: A longitudinal study of corporate sustainable development", Strategic Management Journal, Vol. 26, No. 3, pp. 197-218.

Bansal, P. \& Roth, K. (2000), "Why companies go green: A model of ecological responsiveness", The Academy of Management Journal, Vol. 43, No. 4, pp. 717-736.

Barnett, M. L. (2007), "Stakeholder influence capacity and the variability of financial returns to corporate social responsibility", Academy of Management Review, Vol. 32, No. 3, pp. 794-816.

Barney, J. (1991), "Firm resources and sustained competitive advantage", Journal of Management, Vol. 17, No. 1, pp. 99-120.

Barney, J. B. (2001), "Resource-based theories of competitive advantage: A ten-year retrospective on the resource-based view", Journal of Management, Vol. 27, No. 6, pp. 643-650.

Barney, J. B., \& Clark, D. N. (2007), Resource-based theory: Creating and sustaining competitive advantage, Oxford University Press, Oxford, UK.

Barreto, I., Baden-Fuller, C. \& de Cima, P. (2006), "To conform or to perform? Mimetic behaviour, legitimacy-based groups and performance consequences", Journal of Management Studies, Vol. 43, No. 7, pp. 1559-1581. 
Baxter, J. and Chua, W. F. (2003), "Alternative management accounting research - whence and whither", Accounting, Organizations and Society, Vol. 28, No. 2-3, pp. 97-126.

Bebbington, J. \& Larrinaga-Gonzalez, C. (2008), "Carbon trading: Accounting and reporting issues", European Accounting Review, Vol. 17, No. 4, pp. 697-717.

Berrone, P., Gelabert, L., Fosfuri, A. \& Gomez-Mejia, L. R. (2007), "Can institutional forces create competitive advantage? An empirical examination of environmental innovation". IESE Business School Working Paper No. 723.

Bourgeois III, L. J. \& Eisenhardt, K. M. (1988), "Strategic decision processes in high velocity environments: Four cases in the microcomputer industry", Management Science, Vol. 34, No. 7, pp. 816-835.

Bowen, F. E. (2000), "Environmental visibility: A trigger of green organizational response?", Business Strategy and the Environment, Vol. 9, No. 2, pp. 92-107.

Bowman, E. H. \& Haire, M. (1975), "A strategic posture toward corporate social responsibility", California Management Review, Vol. 18, No. 2, pp. 49-58.

Brammer, S. \& Pavelin, S. (2004), "Voluntary social disclosures by large UK companies", Business Ethics: A European Review, Vol. 13, No. 2-3, pp. 86-99.

Brammer, S. \& Pavelin, S. (2006), "Voluntary environmental disclosures by large UK companies", Journal of Business Finance \& Accounting, Vol. 33, No. 7-8, pp. 11681188.

Brammer, S. \& Pavelin, S. (2008), "Factors influencing the quality of corporate environmental disclosure", Business Strategy and the Environment, Vol. 17, No. 2, pp. 120-136.

Brint, S. \& Karabel, J. (1991), "Institutional origins and transformations: The case of American community colleges", Powell, W. W. \& DiMaggio, P. J., The new institutionalism in organizational analysis. University of Chicago Press, Chicago, pp. 337-360.

Brown, R. (2009), Your business and those 2020 targets, New Zealand Business, August 2009, p. 62.

Brownell, P. (1985), "Budgetary systems and the control of functionally differentiated organizational activities", Journal of Accounting Research, Vol. 23, No. 2, pp. 502-512.

Brownlee, G. (2008), Press release: Thermal Ban Repeal, Tuesday, 16 December 2008, New Zealand Government. Available from: http://www.scoop.co.nz/stories/PA0812/S00193.htm

Brunsson, N. (1989), The organization of hypocrisy, Wiley, New York.

Bungay, S. \& Goold, M. (1991), "Creating a strategic control system", Long Range Planning, Vol. 24, No. 3, pp. 32-39.

Burchell, S., Clubb, C., Hopwood, A., Hughes, J. \& Nahapiet, J. (1980), "The roles of accounting in organizations and society", Accounting, Organizations and Society, Vol. 5, No. 1, pp. 5-27.

Burns, J. \& Scapens, R. W. (2000), "Conceptualizing management accounting change: An institutional framework", Management Accounting Research, Vol. 11, No. 1, pp. 3-25.

Carruthers, B. G. (1995), "Accounting, ambiguity, and the new institutionalism", Accounting, Organizations and Society, Vol. 20, No. 4, pp. 313-328.

Céspedes-Lorente, J., Burgos-Jiménez, J. \& Álvarez-Gil, M. (2003), "Stakeholders' environmental influence. An empirical analysis in the Spanish hotel industry", Scandinavian Journal of Management, Vol. 19, No. 3, pp. 333-358.

Chapman, C. S. (1998), "Accountants in organisational networks", Accounting, Organizations and Society, Vol. 23, No. 8, pp. 737-766.

Chapman, C. S. (2005), Controlling strategy: Management, accounting, and performance measurement, Oxford University Press, New York.

Chenhall, R. H. (2003), "Management control systems design within its organizational context: Findings from contingency-based research and directions for the future", Accounting, Organizations and Society, Vol. 28, No. pp. 127-168. 
Christopher, W. F. (1977), "Achievement reporting--controlling performance against objectives", Long Range Planning, Vol. 10, No. 5, pp. 14-24.

Christensen, M. (2005), "The 'third hand': Private sector consultants in public sector accounting change", European Accounting Review, Vol. 14, No. 3, pp. 447 - 474.

Clark, B. R. (1960), The open-door colleges: A case study, McGraw-Hill, New York.

Clark, B. R. (1972), "The organizational saga in higher education", Administrative Science Quarterly, Vol. 17, No. pp. 178-184.

Clark, H. (2007), Launch of emission trading scheme, 20 September 2007. http://www.beehive.govt.nz/node/30691.

Claver, E., López, M. D., Molina, J. F. \& Tarí, J. J. (2007), "Environmental management and firm performance: A case study", Journal of Environmental Management, Vol. 84, No. 4, pp. 606-619.

Clemens, B. (1997), "On environmental intervention and management strategies", PhD thesis, University of Tennessee, Knoxville, TN.

Coase, R. (1937), "The nature of the firm", Economica, Vol. 4, No. 16, pp. 386-405.

Cole, M. A., Elliott, R. J. R. \& Shimamoto, K. (2006), "Globalization, firm-level characteristics and environmental management: A study of Japan", Ecological Economics, Vol. 59, No. 3, pp. 312-323.

Collins, E., Lawrence, S., Pavlovich, K. \& Ryan, C. (2007), "Business networks and the uptake of sustainability practices: The case of New Zealand", Journal of Cleaner Production, Vol. 15, No. 8-9, pp. 729-740.

Commons, J. R. (1934), Institutional economics, its place in political economy, Macmillan, New York.

Connor, T. (2002), "The resource-based view of strategy and its value to practising managers", Strategic Change, Vol. 11, No. 6, pp. 307-316.

Covaleski, M. \& Dirsmith, M. (1983), "Budgeting as a means for control and loose coupling", Accounting, Organizations and Society, Vol. 8, No. 4, pp. 323-340.

Covaleski, M. A. \& Dirsmith, M. W. (1988), "An institutional perspective on the rise, social transformation, and fall of a university budget category", Administrative Science Quarterly, Vol. 33, No. 4, pp. 562-587.

Covaleski, M. \& Dirsmith, M. (1990), "Dialectic tension, double reflexivity and the everyday accounting researcher: On using qualitative methods", Accounting, Organizations and Society, Vol. 15, No. 6, pp. 543-473.

Covaleski, M. A., Dirsmith, M. W. \& Michelman, J. E. (1993), "An institutional theory perspective on the DRG framework, case-mix accounting systems and health-care organizations", Accounting, Organizations and Society, Vol. 18, No. 1, pp. 65-80.

Covaleski, M. A., Dirsmith, M. W. \& Samuel, S. (2003), "Changes in the institutional environment and the institutions of governance: Extending the contributions of transaction cost economics within the management control literature", Accounting, Organizations and Society, Vol. 28, No. 5, pp. 417-441.

de Bakker, F. G. A., Fisscher, O. A. M. \& Brack, A. J. P. (2002), "Organizing product-oriented environmental management from a firm's perspective", Journal of Cleaner Production, Vol. 10, No. 5, pp. 455-464.

Deephouse, D. (1996), "Does isomorphism legitimate?", Academy of Management Journal, Vol. 39, No. 4, pp. 1024-1039.

Deephouse, D. (1999), "To be different, or to be the same? It's a question (and theory) of strategic balance", Strategic Management Journal, Vol. 20, No.2, pp. 147-166.

Deloitte (2007), "The Risk Intelligent Energy company: Weathering the Storm of Climate Change", Risk Intelligence Series, Issue No.7, Deloitte Global.

Denzin, N. \& Lincoln, Y. (2003), The landscape of qualitative research: Theories and issues, Sage Publications, Thousands Oaks, CA. 
Denzin, N. (2009), The research act: A theoretical introduction to sociological methods, Aldine Transactions Publishers, Piscataway, NJ.

DiMaggio, P. \& Powell, W. W. (1983), "The iron cage revisited: Institutional isomorphism and collective rationality in organizational fields", American Sociological Review, Vol. 48, No. 2, pp. 147-160.

DiMaggio, P. \& Powell, W. W. (1991), The new institutionalism in organizational analysis, University of Chicago Press, Chicago.

DiMaggio, P. (1988), "Interest and agency in institutional theory", Zucker, L. G., Institutional patterns and organizations: Culture and environment. Ballinger, Cambridge, pp. 3-21.

Donaldson, L. (2001), The contingency theory of organizations, Sage, Thousand Oaks, California.

Doonan, J., Lanoie, P. \& Laplante, B. (2005), "Determinants of environmental performance in the Canadian pulp and paper industry: An assessment from inside the industry", Ecological Economics, Vol. 55, No. 1, pp. 73-84.

Dougherty, D. (1994), "Commentary", Shrivastava, P., Huff, A. \& Dutton, P. J., Advances in strategic management. JAI Press, Greenwich, CT, pp. 107-112.

Dutton, J. E. \& Duncan, R. B. (1987), "The creation of momentum for change through the process of strategic issue diagnosis", Strategic Management Journal, Vol. 8, No. 3, pp. 279-295.

EC (2006), "Industry", Electricity Commission, Wellington, Available at http://www.electricitycommission.govt.nz/industry (accessed 4 August 2007).

Eckstein, H. (1975), "Case study and theory in political science", Greenstein, F. \& Polsby, N., Handbook of political science. Addison-Wesley, Reading, MA, pp. 79-137.

Eiadat, Y., Kelly, A., Roche, F. \& Eyadat, H. (2008), "Green and competitive? An empirical test of the mediating role of environmental innovation strategy", Journal of World Business, Vol. 43, No. 2, pp. 131-145.

Eisenhardt, K. M. (1989), "Building theories from case study research", Academy of Management Review, Vol. 14, No. 4, pp. 532-551.

Emissions Trading Group (2007), Regulatory impact statement: A proposed New Zealand emissions trading scheme, Emissions Trading Group, Wellington.

Evans, L. T. \& Meade, R. B. (2005), Alternating currents or counter-revolution?: Contemporary electricity reform in New Zealand, Victoria University Press, Wellington.

Ezzamel, M. (1990), "The impact of environmental uncertainty, managerial autonomy and size on budget characteristics", Management Accounting Research, Vol. 1, No. 3, pp. 181197

Fernández-Alles, M. D. L. L. \& Valle-Cabrera, R. (2006), "Reconciling institutional theory with organizational theories", Management, Vol. 19, No. 4, pp. 503-517.

Ferreira, L. D. \& Merchant, K. A. (1992), "Field research in management accounting and control: A review and evaluation", Accounting, Auditing and Accountability Journal, Vol. 5, No. 4, pp. 3-34.

Fielding, N. \& Lee, R. (1991), Using computers in qualitative research, Sage, London.

Fiol, C. M. (2001), "Revisiting an identity-based view of sustainable competitive advantage", Journal of Management, Vol. 27, No. 6, pp. 691-699.

Flamholtz, E. G., Das, T. K. \& Tsui, A. S. (1985), "Toward an integrative framework of organizational control", Accounting, Organizations and Society, Vol. 10, No. 1, pp. 3550.

Flick, U. (1992), "Triangulation revisited: Strategy of validation or alternative?", Journal for the Theory of Social Behaviour, Vol. 22, No. 2, pp. 175-197.

Flick, U. (2009), An introduction to qualitative research, Sage, London.

Flick, U., Kardorff, E. V. \& Steinke, I. (2004), A companion to qualitative research, Sage, London. 
Fligstein, N. (1997), "Social skill and institutional theory", American behavioral scientist, Vol. 40, No. 4, pp. 397-405.

Fowler, C. (2009), "Performance management, budgeting, and legitimacy in primary educational organisations", Journal of Accounting and Organisational Change, Vol. 5, No. 2, pp. 168-196.

Garriga, E. \& Melé, D. (2004), "Corporate social responsibility theories: Mapping the territory", Journal of Business Ethics, Vol. 53, No. 1, pp. 51-71.

Garrod, B. \& Chadwick, P. (1996), "Environmental management and business strategy: Towards a new strategic paradigm", Futures, Vol. 28, No. 1, pp. 37-50.

Goodrick, E. \& Salancik, G. (1996), "Organizational discretion in responding to institutional practices: Hospitals and Cesarean births", Administrative Science Quarterly, Vol. 41, No. 1, pp. 1-28.

Goodstein, J. D. (1994), "Institutional pressures and strategic responsiveness: Employer involvement in work-family issues", The Academy of Management Journal, Vol. 37, No. 2, pp. 350-382.

Govindarajan, V. (1988), "A contingency approach to strategy implementation at the businessunit level: Integrating administrative mechanisms with strategy", Vol. 31, No. 4, pp. 828-853.

Granlund, M. \& Lukka, K. (1998), "It's a small world of management accounting practices", Journal of Management Accounting Research, Vol. 10, pp. 153-180.

Greening, D. W. \& Gray, B. (1994), "Testing a model of organizational response to social and political issues", The Academy of Management Journal, Vol. 37, No. 3, pp. 467-498.

GreenPeace NZ (2007), Press Release: Electricity companies rated on climate change, 16 July 2007.

Greenwood, R. \& Hinings, C. R. (1996), "Understanding radical organizational change: Bringing together the old and the new institutionalism", Academy of Management Review, Vol. 21, No. 4, pp. 1022-1054.

Gupta, M. C. (1995), "Environmental management and its impact on the operations function", International Journal of Operations \& Production Management, Vol. 15, No. 8, pp. 3451.

Hammersley, M. (1992), What's wrong with ethnography: Methodological explorations, Routledge, London.

Hart, S. L. (1995), "A natural-resource-based view of the firm", Academy of Management Review, Vol. 20, No. 4, pp. 986-1014.

Helfat, C. E., Finkelstein, S., Mitchell, W., Peteraf, M. A., Singh, H., Teece, D. J., \& Winter, S. G. (2007), Dynamic capabilities: Understanding strategic change in organizations, John Wiley, New York.

Helm, D. (2005), "Economic Instruments and Environmental Policy", The Economic and Social Review, Vol. 36, No. 3, pp 1-24.

Henriques, I. \& Sadorsky, P. (1996), "The determinants of an environmentally responsive firm: An empirical approach", Journal of Environmental Economics and Management, Vol. 30, No. 3, pp. 381-395.

Hillman, A. J., Keim, G. D. \& Schuler, D. (2004), "Corporate political activity: A review and research agenda", Journal of Management, Vol. 30, No. 6, pp. 837-857.

Hirsch, P. (1997), "Review essay: Sociology without social structure: Neoinstitutional theory meets brave new world", American Journal of Sociology, Vol. 102, No. 6, pp. 17021723.

Hodgson, P. (2005), Carbon tax speech, The Beehive, Parliament. Wednesday, 4 May 2005.

Hogan, S. \& Meade, R. (2007), "Vertical integration and market power in electricity markets", Presented at New Zealand Association of economists annual conference 2007, Christchurch, New Zealand.

Hofstede, G. H. (1968), The game of budget control, Tavistock, London. 
Holmes, S. L. (1976), "Executive perceptions of corporate social responsibility", Business Horizons, Vol. 19, No. 3, pp. 34-40.

Hopper, T. \& Armstrong, P. (1991), "Cost accounting, controlling labour and the rise of conglomerates", Accounting, Organizations and Society, Vol. 16, No. 5-6, pp. 405-438.

Hopper, T. \& Hoque, Z. (2006), "Triangulation approaches to accounting research", Hoque, Z., Methodological issues in accounting research. Spiramus Press, pp. 477-483.

Hopper, T., Otley, D. \& Scapens, B. (2001), "British management accounting research: Whence and whither: Opinions and recollections", The British Accounting Review, Vol. 33, No. 3, pp. 263-291.

Hopwood, A. G. (1972), "An empirical study of the role of accounting data in performance evaluation", Journal of Accounting Research, Vol. 10, pp. 156-182.

Hopwood, A. (1990), "Accounting and organisation change", Accounting, Auditing \& Accountability Journal, Vol. 3, No. 1, pp. 7-17.

Hrebiniak, L. G. \& Joyce, W. F. (1985), "Organizational adaptation: Strategic choice and environmental determinism", Administrative Science Quarterly, Vol. 30, No. 3, pp. 336349.

Hunt, C. B. \& Auster, E. R. (1990), "Proactive environmental management: Avoiding the toxic trap", Sloan Management Review, Vol. 31, No. 2, pp. 7-18.

Hutchinson, C. (1996), "Integrating environment policy with business strategy", Long Range Planning, Vol. 29, No. 1, pp. 11-23.

IPENZ (2010), Electricity generation - Achieving New Zealand's objectives, The Institution of Professional Engineers New Zealand Inc, Wellington, March 2010.

Ingram, P. \& Simons, T. (1995), "Institutional and resource dependence determinants of responsiveness to work-family issues", Academy of Management Journal, Vol. 38, No. 5, pp. 1466-1482.

Ittner, C.D. and Larcker, D. F. (1997), "Quality strategy, strategic control systems, and organizational performance", Accounting, Organizations and Society, Vol. 22, No. 3-4, pp. 293-314.

James, P., Ghobadian, A., Viney, H. \& Liu, J. (1999), "Addressing the divergence between environmental strategy formulation and implementation", Management Decision, Vol. 37, No. 4, pp. 338-47.

Jansson, A., Nilsson, F. \& Rapp, B. (2000), "Environmentally driven mode of business development: A management control perspective", Scandinavian Journal of Management, Vol. 16, No. 3, pp. 305-333.

Jick, T. D. (1979), "Mixing qualitative and quantitative methods: Triangulation in action", Administrative Science Quarterly, Vol. 24, No. 4, pp. 602-611.

Jones, T. (1995), "Instrumental stakeholder theory: A synthesis of ethics and economics", Academy of Management Review, Vol. 20, No. 2, pp. 404-437.

Judge, W. Q. \& Douglas, T. J. (1998), "Performance implications of incorporating natural environmental issues into the strategic planning process: An empirical assessment", Journal of Management Studies, Vol. 35, No. 2, pp. 241-262.

Kasurinen, T. (2002), "Exploring management accounting change: The case of balanced scorecard implementation", Management Accounting Research, Vol. 13, No. 3, pp. 323343.

Khandwalla, P. N. (1972), "The effect of different types of competition on the use of management controls", Journal of Accounting Research, Vol. 10, pp. 275-285.

Kerr, S. (2007), Emissions Trading in New Zealand: Managing Economic Risk in the New Zealand Emissions Trading System. Paper prepared for New Zealand Climate Change Policy Dialogue, September 2007. Available from: www.ecoclimate.org.nz/managing_econ_risk.pdf

Kostova, T., Roth, K. \& Dacin, M. (2008), "Note: Institutional theory in the study of multinational corporations: A critique and new directions", The Academy of Management Review, Vol. 33, No. 4, pp. 994-1006. 
Kloot, L. (1997), "Organizational learning and management control systems: Responding to environmental change", Management Accounting Research, Vol. 8, No. 1, pp. 47-73.

Kraaijenbrink, J., Spender, J. \& Groen, A. (2010), "The resources-based view: A review and assessment of its critiques", Journal of Management, Vol. 36, No. 1, pp. 349 - 372.

Kraatz, M. \& Zajac, E. (1996), "Exploring the limits of the new institutionalism: The causes and consequences of illegitimate organizational change", American sociological review, Vol. 61, No. 5, pp. 812-836.

Langfield-Smith, K. (1997), "Management control systems and strategy: A critical review", Accounting, Organizations and Society, Vol. 22, No. 2, pp. 207-232.

Levi, M. (1990), "A logic of institutional change", Cook, K. S. \& Levi, M., The limits of rationality. University of Chicago Press, Chicago and London, pp. 402-418.

Lieberman, M. \& Asaba, S. (2006), "Why do firms imitate each other?", Academy of Management Review, Vol. 31, No. 2, pp. 366-395.

Lillis, A. \& Mundy, J. (2005), "Cross-sectional field studies in management accounting research-closing the gaps between surveys and case studies", Journal of Management Accounting Research, Vol. 17, No. 1, pp. 119-141.

Lillis, A. (2006), "Reliability and validity in field study research", Hoque, Z., Methodological Issues in Accounting Research: Theories and Methods. Spiramus, London, pp. 461-468.

Lincoln, Y. \& Guba, E. (1985), Naturalistic inquiry, Sage, Thousand Oaks, CA.

Lowe, E. (1971), "On the idea of a management control system: Integrating accounting and management control", Journal of Management Studies, Vol. 8, No. 1, pp. 1-12.

Malina, M. A. \& Selto, F. H. (2001), "Communicating and controlling strategy: An empirical study of the effectiveness of the balanced scorecard", Journal of Management Accounting Research, Vol. 13, No. 1, pp. 47-91.

Marcus, A. A. (1988), "Responses to externally induced innovation: Their effects on organizational performance", Strategic Management Journal, Vol. 9, No. 4, pp. 387402.

Marginson, D. E. W. (1999), "Beyond the budgetary control system: Towards a two-tiered process of management control", Journal of Financial Economics, Vol. 10, No. 3, pp. 203-230.

Masters, M. F. \& Keim, G. D. (1985), "Determinants of PAC participation among large corporations", The Journal of Politics, Vol. 47, No. 4, pp. 1158-1173.

May, T. (2001), Social research: Issues, methods and process ( $2^{\text {nd }}$ ed), Open University Press.

McWilliams, A. \& Siegel, D. (2001), "Corporate social responsibility: A theory of the firm perspective", Academy of Management Review, Vol. 26, No. 1, pp. 117-127.

MED (2006), Draft New Zealand Energy Strategy to 2050: Powering our Future towards a Sustainable Low Emission Energy System, Wellington: Ministry of Economic Development.

MED (2007). Summary Report on Submissions on the Draft New Zealand Energy Strategy to 2050. Wellington, Ministry of Economic Development.

Merchant, K. A. (1984), "Influences on departmental budgeting: An empirical examination of a contingency model", Accounting Organizations and Society, Vol. 9, No. 3-4, pp. 291307.

Merchant, K. A. (1985), "Organizational controls and discretionary program decision making: A field study", Accounting, Organizations and Society, Vol. 10, No. 1, pp. 67-85.

Merchant, K. A. (1990), "The effects of financial controls on data manipulation and management myopia", Accounting Organizations and Society, Vol. 15, No. 4, pp. $297-$ 313.

Meyer, J. W. (1986), "Social environments and organizational accounting", Accounting, Organizations and Society, Vol. 11, No. 4-5, pp. 345-356.

Meyer, J. W. \& Rowan, B. (1977), "Institutionalized organizations: Formal structure as myth and ceremony", American Journal of Sociology, Vol. 83, No. 2, pp. 340-363. 
Meyer, J. W., Scott, W. R. \& Deal, T. E. (1983), "Institutional and technical sources of organizational structure: Explaining the structure of educational organizations", Meyer, J. W. \& Scott, W. R., Organizational environments: Ritual and rationality. Sage Publications, California, pp. 45-67.

MfE (2005a), "Review of climate change policies", Ministry for the Environment, Wellington, Available at http://www.mfe.govt.nz/publications/climate/policy-review-05/policyreview-05.pdf (accessed 1 September 2008).

MfE (2005b), Annual Report on Climate Change Policy Implementation 2004/2005, Ministry for the Environment, Wellington. Available from: http://www.mfe.govt.nz/publications/climate/annual-report-05/annual-report-policyimplementation.html

MfE (2008), "History of climate change policy development", Ministry for the Environment, Wellington. Available at http://www.mfe.govt.nz/issues/climate/policiesinitiatives/history/index.html (accessed 19 September 2008).MfE (2009a), New Zealand's greenhouse gas inventory 1990-2007, Ministry for the Environment, Wellington (15 April 2009).

MfE (2009a), "Projects to reduce emissions (PRE)", Ministry for the Environment, Wellington. Available at http://www.mfe.govt.nz/issues/climate/policiesinitiatives/projects/index.html (accessed 1 August 2009).

MfE (2009b), Emission trading bulletin No. 11. Summary of the proposed changes to the NZ ETS. $\quad$ Retrieved 20 December 2009 from: http://www.mfe.govt.nz/publications/climate/emissions-trading-bulletin-11/index.html.

MfE (2009c), New Zealand's Greenhouse Gas Inventory 1990-2008. Wellington, Ministry for the Environment. Available from: http://www.mfe.govt.nz/publications/climate/greenhouse-gas-inventory2010/greenhouse-gas-inventory-2010.pdf

MfE (2009d), \# Results of the first projects tender round (2003) and the second projects tender round (2004). Wellington, Ministry for the Environment. Available from http://www.mfe.govt.nz/issues/climate/policies-initiatives/projects/table-successfulprojects-2003.html (Accessed 15 November 2009).

MfE (2010), Greenhouse Gas Emissions. Available from http://www.med.govt.nz/templates/ContentTopicSummary_23719.aspx. Retrieved 30 January 2010.

Miles, M. B. \& Huberman, A. M. (1994), Qualitative data analysis: An expanded sourcebook, Sage, Thousand Oaks.

Miller, P. (1991), "Accounting innovation beyond the enterprise: Problematizing investment decisions and programming economic growth in the U.K in the 1960s", Accounting, Organizations and Society, Vol. 16, No. 8, pp. 733-762.

Miller, P. \& O'Leary, T. (1987), "Accounting and the construction of the governable person", Accounting, Organizations and Society, Vol. 12, No. 3, pp. 235-265.

Miller, G., Dingwall, R. \& Murphy, E. (2004), "Using qualitative data and analysis - reflections on organizational research", Silverman, D., Qualitative research: Theory, method and practice ( $2^{\text {nd }}$ ed), Sage, London, pp 325-340.

Miller, P. (1998), "The margins of accounting", European Accounting Review, Vol. 7, No. 4, pp. 605-621.

Milne, M. J., Owen, D. L. \& Tilt, C. A. (2001), "Corporate environmental reporting: Are New Zealand companies being left behind?", University of Auckland Business Review, Vol. 3, No. 2, pp. 24-36.

Milne, M. J., Tregidga, H. M. \& Walton, S. (2003), "The triple bottom line: Benchmarking New Zealand's early reporters", University of Auckland Business Review, Vol. 5, No. 2, pp. 36-50.

Mizruchi, M. S. and Fein, L. C. (1999), "The Social Construction of Organizational Knowledge: A Study of the Uses of Coercive, Mimetic, and Normative Isomorphism", Administrative Science Quarterly, Vol. 44, No. 4, pp 653-683. 
Moll, J., Burns, J. \& Major, M. (2004), "Institutional theory", Hoque, Z., Methodological issues in accounting research: Theories, methods and issues. Spiramus, London, pp. 183-205.

Morse, J., Barrett, M., Mayan, M., Olson, K. \& Spiers, J. (2002), "Verification strategies for establishing reliability and validity in qualitative research", International Journal of Qualitative Methods, Vol. 1, No. 2, pp. 1-19.

Moxie (2007), Solutions Seekers survey. Understanding the market for sustainable living, Moxie Design Group Limited, Wellington. http://www.shapenz.org.nz/_attachments/Seeking_Environmental_Solutions_Report.pdf

Murray, K. \& Stevenson, T. (2004), Analysis of the state of competition and investment and entry barriers to New Zealand's wholesale and retail electricity markets, Electricity Commission, LECG \& TWSCL, Wellington.

Neuendorf, K. A. (2002), The content analysis guidebook, Sage, Thousand Oaks, CA.

NZBCSD (2002), Business opportunities and global climate change, New Zealand Business Council for Sustainable Development, Wellington. Available at: http://www.nzbcsd.org.nz/climatechange/Climate_Change_Guide.pdf

New Zealand Government (2007a), A framework for New Zealand Emissions Trading Scheme, Ministry for the Environment \& The Treasury, Wellington.

New Zealand Government (2007b), New Zealand Climate Change solutions: An overview, Wellington.

New Zealand Government (2008), "National-ACT agreement announced", National Party, Wellington, Available at http://www.national.org.nz/files/agreements/NationalAct_Agreement.pdf (accessed 10 May 2009).

NZIER (2007), Emissions Trading Scheme for New Zealand: Report to Business New Zealand, New Zealand Institute of Economic Research, Wellington.

NZPA (New Zealand Broadcasting Agency) (2009), "Emissions trading scheme passed into law", New Zealand Herald, Available at http://www.nzherald.co.nz/nz/news/article.cfm?c_id=1\&objectid=10611640 (accessed 25 November 2009).

Ogden, S. (1997), "Accounting for organizational performance: The construction of the customer in the privatized water industry", Accounting, Organizations and Society, Vol. 22, No. 6, pp. 529-556.

Orru, M., Biggart, N. W. \& Hamilton, G. G. (1991), "Organizational isomorphism in East Asia: Broadening the new institutionalism", Powell, W. W. \& DiMaggio, P., The new institutionalism in organizational analysis. University of Chicago Press, Chicago, pp. 361-389.

Oliver, C. (1991), "Strategic responses to institutional processes", The Academy of Management Review, Vol. 16, No. 1, pp. 145-179.

Orlitzky, M., Schmidt, F. L. \& Rynes, S. L. (2003), "Corporate social and financial performance: A meta-analysis", Organization Studies, Vol. 24, No. 3, pp. 403-441.

Otley, D. T. (1978), "Budget use and managerial performance", Journal of Accounting Research, Vol. 16, No. 1, pp. 122-149.

Otley, D. T. (1980), "The contingency theory of management accounting: Achievement and prognosis", Accounting, Organizations and Society, Vol. 5, No. 4, pp. 413-428.

Otley, D. T. (1999), "Performance management: A framework for management control systems research", Management Accounting Research, Vol. 10, No. 4, pp. 363-382.

Otley, D., Broadbent, J. \& Berry, A. (1995), "Research in management control: An overview of its development", British Journal of Management, Vol. 6, Issue supplement S1, pp. S31-S44.

Ouchi, W. G. (1979), "A conceptual framework for the design of organizational control mechanisms", Management Science, Vol. 25, No. 9, pp. 833-848.

Parker, D. (2008a), Release: Historic climate change legislation passes, 10 September, 2008. Available from: http://www.beehive.govt.nz/release/historic+climate+change+legislation+passes 
Parker, D. (2008b), Release: 90\% renewable energy target is achievable. 6 March 2008. Available

http://www.beehive.govt.nz/release/90+renewable+energy+target+achievable/

Phillips, D. (2002), "Comparative historical studies in education: Problems of periodisation reconsidered", British Journal of Educational Studies, Vol. 50, No. 3, pp. 363-377.

Phillips, N. Lawrence, T.B. and Hardy, C. (2004), "Discourse and Institutions", Academy of Management Review, Vol. 29, No. 4, pp. 635-652.

Poole, M. S. and Van de Ven, A. H. (2004), Handbook of organizational change and innovation, Oxford University Press, New York.

Porter, M. E. (1980), Competitive strategy, Free Press, New York.

Preston, A. M. (1991), "The "problem" in and of management information systems", Accounting, Management and Information Technologies, Vol. 1, No. 1, pp. 43-69.

Priem, R. L., \& Butler, J. E. (2001), "Is the Resource-Based "View" a Useful Perspective for Strategic Management Research?", Academy of Management Review, Vol. 26, No. 1, pp. 22-40.

PWC (2008), Businesses Must "Carbon-Proof" To Reduce Impact of ETS, NZ Energy \& Environment Business Week. September 17th, 2008.

PWC (2009), Emissions critical, Issue 10, 2009. PriceWaterhouseCoopers, Wellington.

Qian, W. \& Burritt, R. (2008), "The development of environmental management accounting: An institutional view", Schaltegger, S., Bennett, M., Burritt, R. L. \& Jasch, C., Environmental management accounting for cleaner production. Springer, Netherlands, pp. 233-248.

Ribeiro, J. \& Scapens, R. (2006), "Institutional theories in management accounting change", Management, Vol. 3, No. 2, pp. 94-111.

Richardson, A. J. (1987), "Accounting as a legitimating institution", Accounting, Organizations and Society, Vol. 12, No. 4, pp. 341-355.

Roberts, P. W. \& Greenwood, R. (1997), "Integrating transaction cost and institutional theories: Toward a constrained-efficiency framework for understanding organizational design adoption", The Academy of Management Review, Vol. 22, No. 2, pp. 346-373.

Russo, M. V. \& Fouts, P. A. (1997), "A resource-based perspective on corporate environmental performance and profitability", The Academy of Management Journal, Vol. 40, No. 3, pp. 534-559.

Scapens, R. (2006), "Understanding management accounting practices: A personal journey", The British Accounting Review, Vol. 38, No. 1, pp. 1-30.

Schram, D. H. \& Steen, G. (2001), The psychology and sociology of literature, Johns Benjamin Publishing, Amsterdam.

Scott, W. R. (2001), Institutions and organizations, Sage, London.

Seale, C. F. (1999), The quality of qualitative research, Sage, London.

Selznick, P. (1957), Leadership in administration, POW, Peterson, Evanston, IL.

ShapeNZ (2009), New Zealanders' Attitudes to Climate Change, New Zealand Business Council for Sustainable Development, Wellington, 1 May 2009.

Sharma, S. (2000), "Managerial interpretations and organizational context as predictors of corporate choice of environmental strategy", The Academy of Management Journal, Vol. 43, No. 4, pp. 681-697.

Sharma, S. \& Vredenburg, H. (1998), "Proactive corporate environmental strategy and the development of competitively valuable organizational capabilities", Strategic Management Journal, Vol. 19, No. 8, pp. 729-753.

Sharma, S. \& Nguan, O. (1999), "The biotechnology industry and strategies of biodiversity conservation: The influence of managerial interpretations and risk propensity", Business Strategy and the Environment, Vol. 8, No. 1, pp. 46-61.

Shetty, Y. K. (1979), "New look at corporate goals", California Management Review, Vol. 22, No. 2, pp. 71-79. 
Silverman, D. (2004), Qualitative research: Theory, method and practice (2), Sage, Thousand Oaks, CA.

Silverman, D. (2006), Interpreting qualitative data: Methods for analysing talk, text, and interaction (3rd ed), Sage Publications, London.

Simons, R. (1990), "The role of management control systems in creating competitive advantage: New perspectives", Accounting Organizations and Society, Vol. 15, No. 1/2, pp. 127143.

Simons, R. (1991), "Strategic orientation and top management attention to control systems", Journal of Financial Economics, Vol. 12, No. 1, pp. 49-62.

Sobel, R. (1984), Car wars, Dutton, New York.

Spekle, R. F. (2001), "Explaining management control structure variety: A transaction cost economics perspective", Accounting, Organizations and Society, Vol. 26, No. 4, pp. 419-441.

Spicer, B. H. \& Ballew, V. (1983), "Management accounting systems and the economics of internal organization", Accounting, Organizations and Society, Vol. 8, No. 1, pp. 73-96.

Standards Australia \& Standards New Zealand (2004), Risk management AS/NZS 4360: 2004, Standards Association of Australia.

Steger, U. (1993), "The greening of the board room: How German companies are dealing with environmental issues", Fischer, K. \& Schot, J., Environmental strategy for industry. Island Press, Washington, DC, pp. 147-66.

Stinchcombe, A. (1997), "On the virtues of the old institutionalism", Annual review of sociology, Vol. 23, pp. 1-18.

Tilley, F. (1999), "The gap between the environmental attitudes and the environmental behaviour of small firms", Business Strategy and the Environment, Vol. 8, No. 4, pp. 238-248.

Tolbert, P. S. \& Zucker, L. G. (1983), "Institutional sources of change in the formal structure of organizations: The diffusion of civil service reform, 1880-1935", Administrative Science Quarterly, Vol. 28, No. 1, pp. 22-39.

Tolbert, P.S and Zucker, L. G. (1996), The institutionalization of institutional theory, S.R. Clegg, C. Hardy, and W.R Nord, Handbook of organizational studies. Sages, Thousands Oaks, CA, pp. 175-190.

Tomkins, B. (2009), "Your carbon footprint may be sign in the sand", National business Review, 20/03/2009, p. 25.

Trevett, C. \& Bradley, G. (2009), "Minister's get-tough letter puts heat on SOEs", The New Zealand Herald, 14 March 2009. Available from: http://www.nzherald.co.nz/nz/news/article.cfm?c_id=1\&objectid=10561649

Tsamenyi, M., Cullen, J., González, J. M. (2006), "Changes in accounting and financial information system in a Spanish electricity company: A new institutional theory analysis", Management Accounting Research, Vol. 17, No. 4, pp 409-432.

Tyagi, S. (2009), "Navigating the carbon world", National Business Review, Section: Business, p. 40, 5 June 2009.

Van Wassenhove, L. N. \& Corbett, C. (1991), How green is your manufacturing strategy, INSEAD R\&D, Working Paper no. 91/50/TM/SM.

Walker, M. (1998), "Management accounting and the economics of internal organization: A review essay", Management Accounting Research, Vol. 9, No. 1, pp. 21-30.

Wallander, J. (1999), "Budgeting. An unnecessary evil", Scandinavian Journal of Management, Vol. 15, No. 4, pp. 405-21.

Warren, C. A. B. (2002), "Qualitative interviewing", Gubrium, J. \& Holstein, J., Handbook of interview research: Context and meaning. Sage, Thousand Oaks, CA, pp. 83-101.

Watts, R. L. \& Zimmerman, J. L. (1986), Positive accounting theory, Prentice-Hall, Englewood Cliffs. 
Weiss, R. S. (1994). Learning from strangers: The art and method of qualitative interview studies. New York: Free Press.

Westley, F. \& Vrendenburg, H. (1991), "Strategic bridging: The collaboration between environmentalists and business in the making of green products", Journal of Applied Behavioral Science, Vol. 27, No. 1, pp. 65-90.

Wickramasinghe, D., Hopper, T. \& Rathnasiri, C. (2004), "Japanese cost management meets Sri Lankan politics: Disappearance and reappearance of bureaucratic management controls in a privatised utility", Accounting, Auditing \& Accountability Journal, Vol. 17, No. 1, pp. 85-120.

Williamson, O.E. (1975), Markets and hierarchies, The Free Press, New York, NY.

Williamson, O. E (1985), The Economic Institutions of Capitalism, $\left(1^{\text {st }} \mathrm{ed}\right)$, The Free Press, New York.

Williamson, O. E. (1991), "Comparative economic organization: The analysis of discrete structural alternatives", Administrative Science Quarterly, Vol. 36, No. 2, pp. 269-296.

Williamson, O. E. (1996), The mechanisms of governance, Oxford University Press, USA.

Williamson, O. E. (1999), "Strategy research: Governance and competence perspectives", Strategic Management Journal, Vol. 20, No. 12, pp. 1087-1108.

Willits, S. \& Giuntini, R. (1994), "Helping your company go green'", Management Accounting (USA), Vol. 75, No. 8, pp. 43-47.

Yermack, D. (1995), "Do corporations award CEO stock options effectively", Journal of Financial Economics, Vol. 39, No. 2, pp. 237-69.

Yin, R. K. (2003), Case study research: Design and methods, Sage Publications Inc.

Zimmerman, J. L. (1997), Accounting for decision making and control (2ed.), Richard D. Irwin, Chicago, IL.

Zimmerman, J. L. (2001), "Conjectures regarding empirical managerial accounting research", Journal of Accounting and Economics, Vol. 32, No. 1-3, pp. 411-427. 